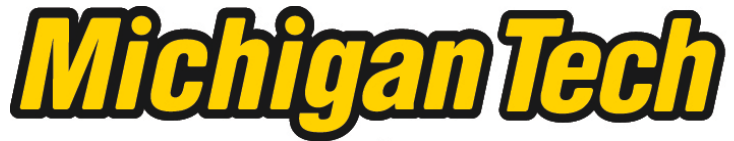 \\ Michigan Technological University Create the Future Digital Commons @ Michigan Tech
}

Dissertations, Master's Theses and Master's Reports - Open

Dissertations, Master's Theses and Master's

Reports

2010

Growth, modification and integration of carbon nanotubes into molecular electronics

Jason P. Moscatello

Michigan Technological University

Follow this and additional works at: https://digitalcommons.mtu.edu/etds

Part of the Physics Commons

Copyright 2010 Jason P. Moscatello

\section{Recommended Citation}

Moscatello, Jason P., "Growth, modification and integration of carbon nanotubes into molecular electronics", Dissertation, Michigan Technological University, 2010.

https://doi.org/10.37099/mtu.dc.etds/112

Follow this and additional works at: https://digitalcommons.mtu.edu/etds

Part of the Physics Commons 


\title{
Growth, Modification and Integration of Carbon Nanotubes into Molecular Electronics
}

\author{
by \\ Jason P. Moscatello
}

A dissertation in partial fulfillment of the requirements for the degree of Doctor of Philosophy (Engineering Physics)

Department of Physics Michigan Technological University

2010 
This dissertation, "Growth, Modification and Integration of Carbon Nanotubes into Molecular Electronics," is hereby approved in partial fulfillment of the requirements for the degree of Doctor of Philosophy in the field of Engineering Physics.

Department: Physics

Research Advisor and

Chair of Advisory Committee:

Dr. Yoke Khin Yap

Committee Members:

Dr. Craig Friedrich

Dr. Ravindra Pandey

Dr. Bryan Suits

Physics Department Chair:

Dr. Ravindra Pandey

Date: 


\section{Abstract}

Molecules are the smallest possible elements for electronic devices, with active elements for such devices typically a few Angstroms in footprint area. Owing to the possibility of producing ultrahigh density devices, tremendous effort has been invested in producing electronic junctions by using various types of molecules. The major issues for molecular electronics include (1) developing an effective scheme to connect molecules with the present micro- and nano-technology, (2) increasing the lifetime and stabilities of the devices, and (3) increasing their performance in comparison to the state-of-the-art devices.

In this work, we attempt to use carbon nanotubes (CNTs) as the interconnecting nanoelectrodes between molecules and microelectrodes. The ultimate goal is to use two individual CNTs to sandwich molecules in a cross-bar configuration while having these CNTs connected with microelectrodes such that the junction displays the electronic character of the molecule chosen. We have success-

fully developed an effective scheme to connect molecules with CNTs, which is scalable to arrays of molecular electronic devices. To realize this far reaching goal, the following technical topics have been investigated.

1. Synthesis of multi-walled carbon nanotubes (MWCNTs) by thermal chemical vapor deposition (T-CVD) and plasma-enhanced chemical vapor deposition (PECVD) techniques (Chapter 3). We have evaluated the potential use of tubular and bamboo-like MWCNTs grown by T-CVD and PE-CVD in terms of their structural properties.

2. Horizontal dispersion of MWCNTs with and without surfactants, and the integration of MWCNTs to microelectrodes using deposition by dielectrophoresis (DEP) (Chapter 4). We have systematically studied the use of surfactant molecules to disperse and 
horizontally align MWCNTs on substrates. In addition, DEP is shown to produce impurityfree placement of MWCNTs, forming connections between microelectrodes. We demonstrate the deposition density is tunable by both AC field strength and AC field frequency.

3. Etching of MWCNTs for the impurity-free nanoelectrodes (Chapter 5). We show that the residual Ni catalyst on MWCNTs can be removed by acid etching; the tip removal and collapsing of tubes into pyramids enhances the stability of field emission from the tube arrays. The acid-etching process can be used to functionalize the MWCNTs, which was used to make our initial CNT-nanoelectrode glucose sensors. Finally, lessons learned trying to perform spectroscopic analysis of the functionalized MWCNTs were vital for designing our final devices.

4. Molecular junction design and electrochemical synthesis of biphenyl molecules on carbon microelectrodes for all-carbon molecular devices (Chapter 6). Utilizing the experience gained on the work done so far, our final device design is described. We demonstrate the capability of preparing patterned glassy carbon films to serve as the bottom electrode in the new geometry. However, the molecular switching behavior of biphenyl was not observed by scanning tunneling microscopy (STM), mercury drop or fabricated glassy carbon/biphenyl/MWCNT junctions. Either the density of these molecules is not optimum for effective integration of devices using MWCNTs as the nanoelectrodes, or an electroactive contaminant was reduced instead of the ionic biphenyl species.

5. Self-assembly of octadecanethiol (ODT) molecules on gold microelectrodes for functional molecular devices (Chapter 7). We have realized an effective scheme to produce Au/ODT/MWCNT junctions by spanning MWCNTs across ODT-functionalized microelectrodes. A percentage of the resulting junctions retain the expected character of an ODT monolayer. While the process is not yet optimized, our successful junctions show that molecular electronic devices can be fabricated using simple processes such as photolithography, self-assembled monolayers and dielectrophoresis. 
Dedicated to the loving memory of my grandmother

\section{Edith M. Blake}

$1921-2010$

and my great aunt

Dorcas D. Manrodt

$1930-2008$ 


\section{Acknowledgments}

I could not have made it as far as I have without the support of countless people, so it would be foolish to try to name them all. Nevertheless, I will endeavor to name a small portion of them here.

I am grateful to Dr. Yoke Khin Yap for allowing me to enter the world of nanotechnology, and for trusting I had the ability to work on a project such as this one. My thanks go out to my committee members, Dr. Ravindra Pandey, Dr. Bryan Suits and Dr. Craig Friedrich, for taking the time to guide me along my path of learning, for asking questions that made me think and for the suggestions along the way. I could not have done this without the support and assistance of my fellow researchers, Benjamin Ulmen, Vijaya Kayastha, Jiesheng Wang, Samuel Mensah, Ming Xie, Archana Pandey, Abhishek Prasad, Chee Huei Lee, Abhay Singh, Shun Wu, Ravi Chintala and Vamsi Kunapuli. I am indebted to the MTU Physics Department for their continual support and guidance, with special thanks to Dr. Donald Beck (for patiently putting up with my endless homework questions).

My work is interdisciplinary and collaborative, and there are many from outside who have helped me along the way. My deepest thanks to Dr. Shashi Karna and Dr. Govind Mallick for allowing me to learn within their laboratory at the ARL and from their very knowledgeable persons. Dr. Friedrich's group at Michigan Tech played a very large hand in this work, from allowing the use of their photomask (which was vital), to collaborating with Libao An, and spending time in the classroom and cleanroom with Mark Griep, Christopher Anton and Karl Walczak. Special thanks are in order to those who have contributed a great amount of time mentoring and teaching their fellow students, thank you Raghav Vanga, Kumar Vanga, Dawdon Cheam and Karl Walczak.

My journey as a physicist did not begin at MTU. My parents, Elizabeth and Peter, always encouraged my questions and learning and helped my young mind flourish, as did my whole family. 
In my younger years, Mrs. Proud at Rose M. Patania did wonders to provoke thought and discussion, and were it not for Mr. Donnelly of Boonton High School, I may have chosen another discipline. While science had always interested me, Mr. Donnelly's knowledge and charm opened my eyes to how much more I could know, and how much more I could help discover. Dr. Romulo Ochoa at The College of New Jersey mentored me, introduced me to laboratory research, and was always encouraging me to aim higher; he remains the only professor I regularly discuss videogames with. I will always be indebted to Dr. Marc Sher of The College of William \& Mary for taking a chance on me - I hope I have made you proud. Thank you to Dr. Gina Hoatson for allowing me the chance to learn and work within her laboratory.

If not for my family and friends, I would be no one. Thank you to my parents, and to my sister Jill for putting up with me for all these years.

My volleyball buddies here at MTU keep me sane and healthy, thank you for coming out week after week just because I like volleyball! Now, about that soccer match... Broomball team: good inaugural season! It was an excellent experience; soldier on next year without me, but do not forget about me. Colin Gurganus, Kris Bunker, Matt Beals, Matt Holinger and Lakshmi Krishna have all contributed to my happiness at MTU and, thus, my productiveness. Benjamin Ulmen deserves special thanks; if not for Ben I would have gotten only $1 / 2$ as far as I have, and it would have been only $1 / 2$ as much fun.

My time spent at The College of William \& Mary provided me with some of the best friends I have very made, many of whom suffered beside me during our marathon quantum homework sessions and the qualifying exams. This is to say nothing of having had the chance to help found our excellent floor hockey team and the tradition of Halo nights up on the projection screens (I may have had something to do with starting the parties, too). I have the most heartfelt thanks and gratitude for Sarah Phillips, Justin Conroy, Allyn Powell, Joshua Moss, Melanie Collins, Vincent Sulkosky, Dan Steiner, Christopher Kulp, Aidan Kelleher, David Anderson, Dandan Mao, Bryan Moffit, Keoki Seu, Meghan Goldman and Shannon Watson.

At The College of New Jersey I made two of my most enduring friends whose encouragement has been a great asset. Thank you, Mr. Andrew Feldman for indulging and adding to my madness and creativity, and for being steadfastly loyal even after all these years (of me not calling as often as I should). Michelle Reno, fellow physicist, fellow random person, thank you for being such a good 
friend who I can always connect to, no matter how long it has been.

Wil Slough deserves special thanks for helping me grow as a person, as a physicist, as a cinephile and in countless other ways. Thank you, Wil, for your advice, your stories, your support and your friendship. To my oldest friends I owe everything, and my success is their success. Thank you, Nicholas Rigano, for being someone I know I can count on, because you prove it time and time again. Thank you for opening my mind up to reading, and to other creative endeavors. Thank you Dan Zugale for being my musical ally (though you have long since outstripped my talent), and for inspiring me with your own hard work ethic. Thank you to Jason Kalivas, one of the constants in my life. Your support, your enthusiasm, your friendship and the ludicrous number of stories we have accrued and experienced over the years have helped me in so many ways I cannot properly express them.

Most of all, my undying love and gratitude to Sarah Phillips. None of this would have been possible without your love and friendship; thank you for walking with me in this life. 


\section{Contents}

$\begin{array}{ll}\text { Abstract } & \text { iii }\end{array}$

Acknowledgments $\quad$ vi

List of Figures $\quad$ xii

List of Tables $\quad$ xvi

Units and Abbreviations $\quad$ xviii

1 Research Overview 1

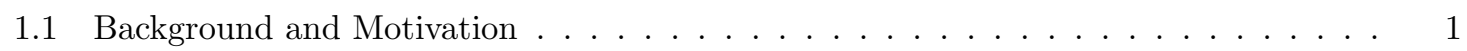

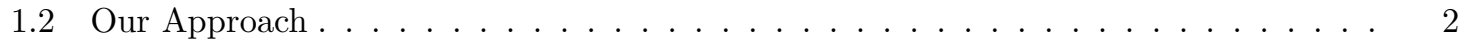

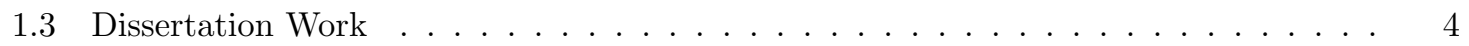

2 Introduction to Carbon Nanotubes $\quad 6$

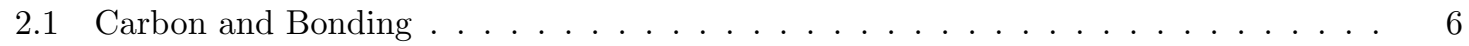

2.2 Carbon Nanotubes Discovery . . . . . . . . . . . . . . . . . . . . . . . . . 11

2.3 Carbon Nanotubes Structures . . . . . . . . . . . . . . . . . . . . . . . . . . 12

2.4 Carbon Nanotubes Growth _. . . . . . . . . . . . . . . . . . . . . . 14

3 Carbon Nanotube Growth 16

3.1 Thermal Chemical Vapor Deposition Process _ . . . . . . . . . . . . . . . 16

3.1.1 Deposition of the Catalyst Film . . . . . . . . . . . . . . . . 16

3.1.2 Pretreatment of the Catalyst Films . . . . . . . . . . . . . . . . . . 19 
3.1.3 Carbon Nanotube Growth and Mechanism . . . . . . . . . . . . . . . 20

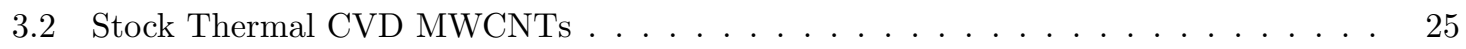

3.3 Bamboo-like Multi-walled Carbon Nanotubes . . . . . . . . . . . . . . . . . . 27

3.4 Plasma-Enhanced CVD Growth and Stock MWCNTs . . . . . . . . . . . . . 33

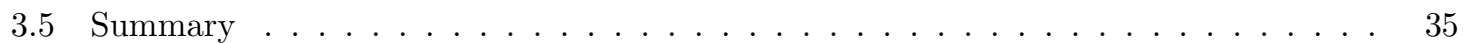

4 Horizontal Dispersions \& Dielectrophoretic Deposition $\quad 38$

4.1 Horizontal Dispersion . . . . . . . . . . . . . . . . . . . . . . . 38

4.1 .1 Introduction . . . . . . . . . . . . . . . . . . 38

$4.1 .2 \quad$ Mechanism and Goals . . . . . . . . . . . . . . . . . . . . . . 39

4.1 .3 Effect of SDS Concentration . . . . . . . . . . . . . . . . . . 40

4.1.4 Effect of Dispersion Method . . . . . . . . . . . . . . . . . . . . . 42

4.1.5 Surfactant-free Dispersions _. . . . . . . . . . . . . . . . . . . . 44

4.2 Dielectrophoretic Deposition . . . . . . . . . . . . . . . . . . . . . . . . 49

4.2 .1 Background Theory . . . . . . . . . . . . . . . . . 49

$4.2 .2 \quad$ Research Goals and Collaborators _. . . . . . . . . . . . . . . 55

4.2 .3 Initial DEP Experiments $\ldots \ldots \ldots \ldots \ldots \ldots \ldots$

4.2 .4 Flat Electrode Gaps . . . . . . . . . . . . . . . . . . . . . 58

4.2 .5 Microelectrode Fingers . . . . . . . . . . . . . . . . . . . . . . . . . . . . . . 64

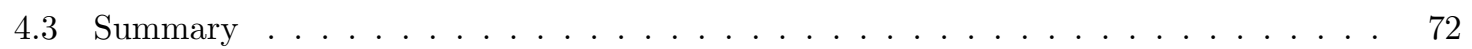

5 Carbon Nanotube Modification and Bundling $\quad 75$

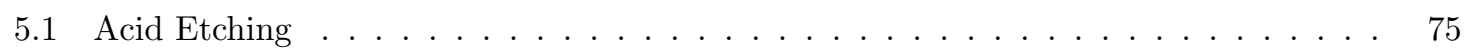

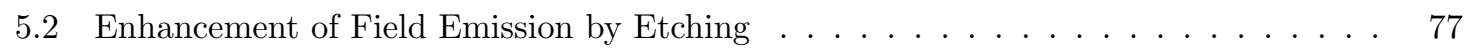

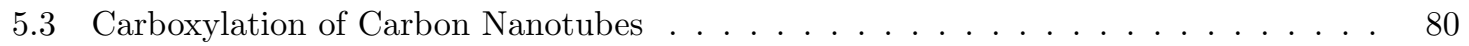

5.4 Glucose Sensors . . . . . . . . . . . . . . . . . . . . . . . . . . . 81

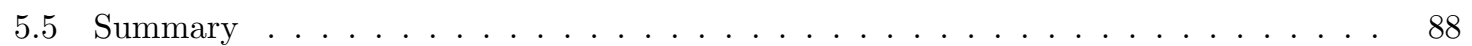

6 Molecular Electronics Devices I $\quad 90$

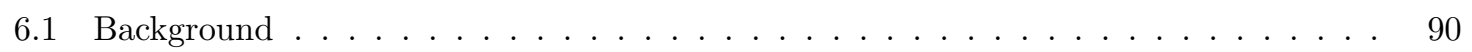

6.2 Motivation and Initial Design . . . . . . . . . . . . . . . . . . . . 91 
6.3 Design Modifications . . . . . . . . . . . . . . . . . . . . . . . 98

6.4 Carbon-Organic-Carbon Approach . . . . . . . . . . . . . . . . . . 101

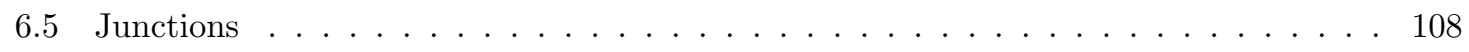

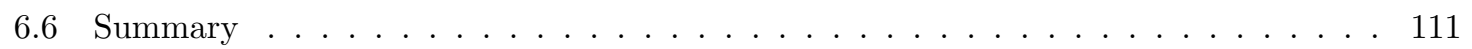

7 Molecular Electronics Devices II $\quad 114$

7.1 Self-Assembled Monolayers . . . . . . . . . . . . . . . . . . . . . . . 114

7.2 Octadecanethiol . . . . . . . . . . . . . . . . . . . . 115

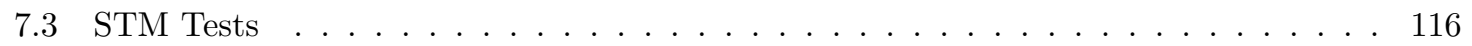

7.4 Initial Patterned ODT Junctions $\ldots \ldots \ldots \ldots \ldots \ldots \ldots \ldots$

7.5 Device Fabrication Improvements . . . . . . . . . . . . . . . . . . . 124

7.5.1 Comparison of Deposition Methods . . . . . . . . . . . . . . . . . . . 124

$7.5 .2 \mathrm{Cr} / \mathrm{Au}$ Annealing . . . . . . . . . . . . . . . . . . . . . 126

7.6 Additional Problems and Film Improvements . . . . . . . . . . . . . . . . . 127

7.7 Device Testing . . . . . . . . . . . . . . . . . . . . . 130

7.8 Summary and Conclusions . . . . . . . . . . . . . . . . . . . . . . . 144

$\begin{array}{lr}\text { A Equipment and Techniques } & 146\end{array}$

A.1 Photolithography . . . . . . . . . . . . . . . . . . . . . . 146

A.1.1 Historical Perspective . . . . . . . . . . . . . . . . . . . 146

A.1.2 Background Information . . . . . . . . . . . . . . . . . . . 147

A.1.3 Our Application . . . . . . . . . . . . . . . . . . . . . . 152

A.2 Raman Spectra . . . . . . . . . . . . . . . . . . . . . . 154

A.3 Scanning Electron Microscope . . . . . . . . . . . . . . . . . 156

A.3.1 Historical Perspective . . . . . . . . . . . . . . . . . . 156

A.3.2 Background . . . . . . . . . . . . . . . . . . . 157

$\begin{array}{ll}\text { B Publications \& Awards } & 160\end{array}$

$\begin{array}{ll}\text { C Permissions } & 162\end{array}$

C.1 American Institute of Physics, I . . . . . . . . . . . . . . . . . . 163

C.2 American Institute of Physics, II _ . . . . . . . . . . . . . . . . 165 
C.3 Archana Pandey . . . . . . . . . . . . . . . . . . . . . . . . . . 168

C.4 Benjamin Ulmen . . . . . . . . . . . . . . . . . . . . . . 169

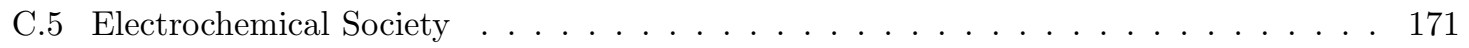

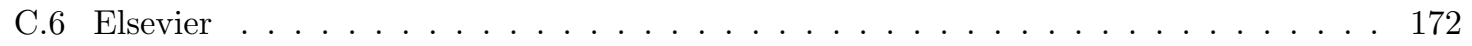

C.6.1 Author's Permission . . . . . . . . . . . . . . . . . . . . . . . 175

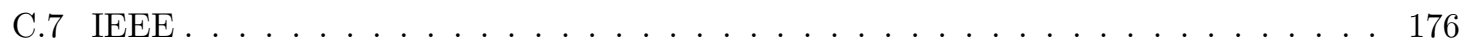

C.8 Imperial College Press . . . . . . . . . . . . . . . . . . . . . . . . 177

C.9 Institute of Physics $\ldots \ldots \ldots \ldots \ldots \ldots \ldots \ldots$

C.9.1 Author's Permission $1 \ldots \ldots \ldots \ldots$

C.9.2 Author's Permission $2 \ldots \ldots \ldots \ldots$

C.10 Ravi Chintala . . . . . . . . . . . . . . . . . . . . . . . . . . 181

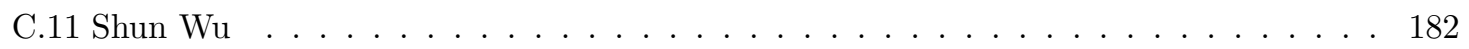

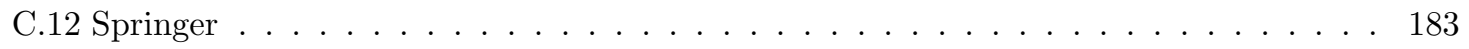

C.13 University of Cambridge . . . . . . . . . . . . . . . . . . . 186 


\section{List of Figures}

1.1 Schematic representation of the original molecular electronics device design . . . . 3

1.2 Dissertation work flow chart . . . . . . . . . . . . . . . . . . . 5

2.1 Visual representation of $s p$ hybridized orbitals . . . . . . . . . . . . . . . . 8

2.2 Visual representation of $s p^{2}$ hybridized orbitals . . . . . . . . . . . . . . . . . . 9

2.3 Planes of graphene stack to form graphite . . . . . . . . . . . . . . . . . 10

2.4 The $s p^{3}$ carbon forms the complex unit cell of diamond . . . . . . . . . . . . . 11

2.5 Folding graphene sheets to form CNTs . . . . . . . . . . . . . . . . . . . . . 13

2.6 Zig-zag SWCNT diameter versus bandgap . . . . . . . . . . . . . . . . . . . . 14

3.1 Schematic of the PLD setup . . . . . . . . . . . . . . . . . . . 18

3.2 The PLD chamber . . . . . . . . . . . . . . . . . . . . . 18

3.3 Schematic of the TCVD setup . . . . . . . . . . . . . . . . . . . . 20

3.4 The TCVD system . . . . . . . . . . . . . . . . . . . . . . . . . 21

3.5 The dissociate adsorption VLS growth mechanism . . . . . . . . . . . . . . . 23

3.6 Base and tip growth . . . . . . . . . . . . . . . . . . . . . . . . . 24

3.7 Stock TCVD MWCNTs . . . . . . . . . . . . . . . . . . . . . 25

3.8 Stock TCVD Raman spectra . . . . . . . . . . . . . . . . . . . . 26

3.9 Effect of temperature on bamboo-like growth . . . . . . . . . . . . . . . . . 29

3.10 Transition temperature from normal growth to bamboo-like growth . . . . . . . . 30

3.11 Ar pretreatment yields no bamboo-like tubes . . . . . . . . . . . . . . . . . 31

3.12 Effect of buffer gases on growth . . . . . . . . . . . . . . . . . . . . 32 
3.13 Bamboo growth on low resistance silicon . . . . . . . . . . . . . . . 33

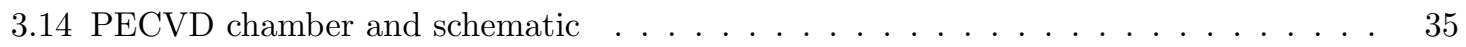

3.15 Stock PECVD grown MWCNTs . . . . . . . . . . . . . . . 36

3.16 Stock PECVD Raman spectra . . . . . . . . . . . . . . . . . . . . . 37

4.1 Chemical structure of sodium dodecyl sulfate . . . . . . . . . . . . . . . 40

4.2 Effect of SDS Concentration of MWCNT Dispersions . . . . . . . . . . . . . . 41

4.3 Comparison of horizontal and vertical MWCNT dispersion methods . . . . . . . . . 42

4.4 Comparison of horizontal and vertical MWCNT dispersion results . . . . . . . . . 43

4.5 MWCNT connections between electrodes made using the vertical dispersion method 45

$4.6 \mathrm{I}-\mathrm{V}$ characteristics of a TCVD grown MWCNT $\ldots \ldots \ldots \ldots \ldots$

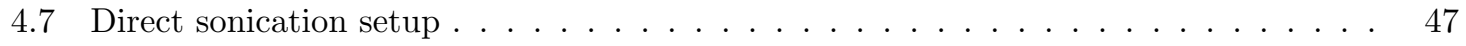

4.8 Under and over sonication of CNT dispersions . . . . . . . . . . . . . . . . 47

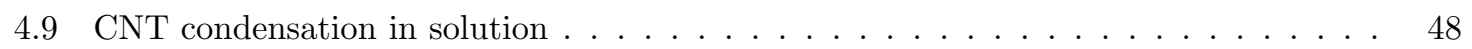

4.10 Heating damage caused by dielectrophoresis in water . . . . . . . . . . . 50

4.11 A dipole in a non-uniform electric field, showing the vectors and labeling. . . . . . . 52

4.12 Example CM factor vs. frequency for spherical particles. . . . . . . . . . . . 56

4.13 First DEP experiment using shadowmasks . . . . . . . . . . . . . . . . 57

4.14 Comparison of CNT deposition with and without AC field . . . . . . . . . 58

4.15 Effect of AC voltage on DEP MWCNT deposition in a flat gap . . . . . . . . . 60

4.16 Effect of varying AC frequency on dielectrophoretic MWCNT deposition in a flat gap 61

4.17 Effect of varying sinusoidal AC voltage on for thermal CVD grown MWCNTs . . . . 62

$4.18 \mathrm{CM}$ factor prediction for stock TCVD tubes in ethanol . . . . . . . . . . . . 63

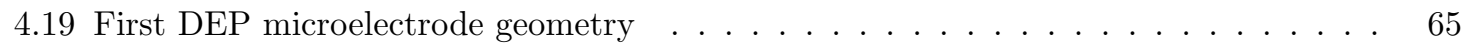

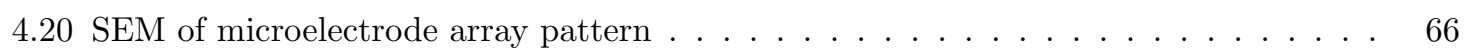

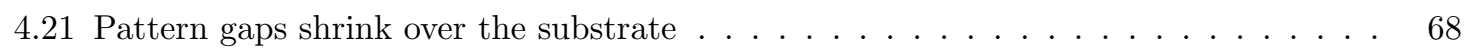

4.22 The improved microelectrode finger array pattern . . . . . . . . . . . . . . 69

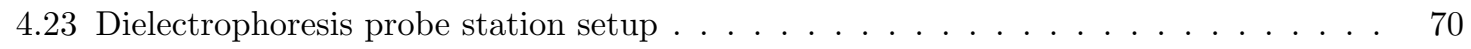

4.24 DEP results for PECVD CNTs on microelectrode finger arrays . . . . . . . . . 71

4.25 DEP results for TCVD CNTs on microelectrode finger arrays . . . . . . . . . 73 


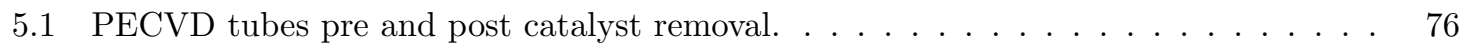

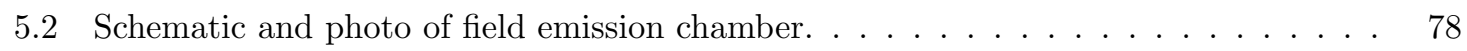

5.3 Field emission stability of as-grown and etched and bundled MWCNTs. . . . . . . . 79

5.4 Simulated potential maps for vertical and bundled MWCNTs. . . . . . . . . . . . . 79

5.5 Photoluminescence spectra of FITC-labeled BSA functionalized MWCNTs. . . . . . 82

5.6 Formation of the MWCNT nanoelectrode array. . . . . . . . . . . . . . . . 84

5.7 Schematic diagram of functionalized array and electrochemical cell. . . . . . . . 85

5.8 Response of glucose sensor to successive additions of glucose. . . . . . . . . . . . 86

5.9 Sensor current response for different glucose concentrations. . . . . . . . . . . . . 87

5.10 Sensor consumption and recovery after glucose addition. . . . . . . . . . . . . . . 89

6.1 Schematic of an molecular junction as studied by McCreery et al. . . . . . . . . . 93

6.2 Current/voltage behaviour of some molecular junctions studied by McCreery et al. . 94

6.3 Schematic cartoon of initial cross-junction design. . . . . . . . . . . . . . . . 95

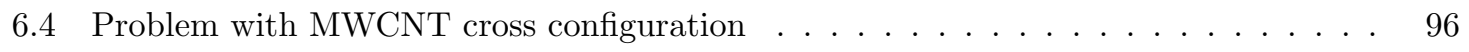

6.5 Submersion in liquid causes CNTs to collapse. . . . . . . . . . . . . . 97

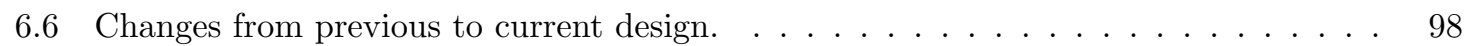

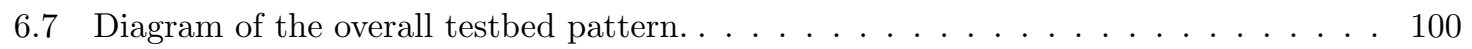

6.8 Testbed device pattern type one. . . . . . . . . . . . . . . . . . . 100

6.9 Testbed device pattern number two. . . . . . . . . . . . . . . . . 101

6.10 Raman spectra of PPF films . . . . . . . . . . . . . . . . . . 103

6.11 Raman spectra of glassy carbon . . . . . . . . . . . . . . . . . . . . . . 104

6.12 Example AFM scan of the PPFs. . . . . . . . . . . . . . . . . 105

6.13 Initial DEP on the device pattern . . . . . . . . . . . . . . . . . 106

6.14 Synthesis of biphenyl diazonium salt . . . . . . . . . . . . . . . . . . 107

6.15 Derivatization scan for $\mathrm{PPF}$ film . . . . . . . . . . . . . . . . 109

6.16 Derivatization scan for PPF device pattern f . . . . . . . . . . . . . . 110

6.17 STM of biphenyl functionalized carbon films. . . . . . . . . . . . . . . . . 112

7.1 Cartoon of the SAM molecule and deposition process. . . . . . . . . . . . . 115

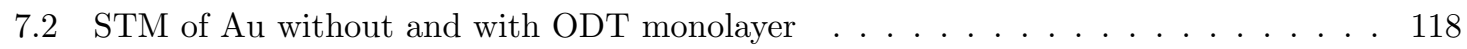


7.3 Schematic profile of the ODT double junction device . . . . . . . . . . . . . 119

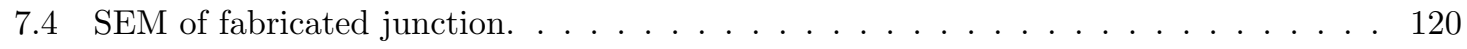

$7.5 \mathrm{I}-\mathrm{V}$ of complete initial junction $\ldots \ldots \ldots \ldots \ldots \ldots \ldots \ldots \ldots$

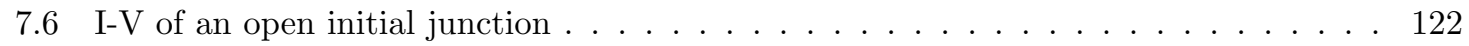

7.7 I-V of a potential ODT junction on the DEP pattern . . . . . . . . . . . . . . . 123

7.8 SEM of lift-off produced Au electrodes, highlighting sharp and rough edges. . . . . . 125

7.9 The envisioned design process for the annealed, etched devices $\ldots \ldots \ldots \ldots$

7.10 Comparison of Au etching on unannealed and annealed $\mathrm{Cr} / \mathrm{Au}$ films $\ldots \ldots$. . . . . 129

7.11 Comparison of different Au films . . . . . . . . . . . . . . . . . . . 129

$7.12 \mathrm{I}-\mathrm{V}$ characteristics of a $100 \times 100 \mu \mathrm{m}$ square pattern showing shorted behaviour $\ldots 132$

$7.13 \mathrm{I}-\mathrm{V}$ characteristics of a $1000 \times 1000 \mu \mathrm{m}$ square pattern showing shorted behaviour .133

$7.14 \mathrm{I}-\mathrm{V}$ characteristics Junction 2 showing a region of minimal current flow and hysteresis 134

7.15 Comparison of shorted and working molecular junctions . . . . . . . . . . . 135

$7.16 \mathrm{I}-\mathrm{V}$ characteristics Junction 2 showing hysteresis $\ldots \ldots \ldots \ldots$

7.17 I-V showing Junction 2 being broken by too much voltage . . . . . . . . . . . . 137

$7.18 \mathrm{I}-\mathrm{V}$ of Junction 2 after breakage . . . . . . . . . . . . . . . . . . 138

$7.19 \mathrm{I}-\mathrm{V}$ of the first 4 scans of the second working junction $\ldots \ldots \ldots \ldots \ldots$

$7.20 \mathrm{I}-\mathrm{V}$ of the last three scans of the second working junction $\ldots \ldots \ldots \ldots$

$7.21 \mathrm{I}-\mathrm{V}$ of the first 7 scans of Junction $3 . \ldots \ldots \ldots \ldots \ldots$

$7.22 \mathrm{I}-\mathrm{V}$ of the second molecular junction two weeks apart . . . . . . . . . . . . . . 142

7.23 Repeated scans at $\pm 4 \mathrm{~V}$ of the second junction . . . . . . . . . . . . . 143

7.24 Repeated scans at $\pm 8 \mathrm{~V}$ of the second junction . . . . . . . . . . . . . . 144

A.1 Example photolithographic process . . . . . . . . . . . . . . . . . . 148

A.2 Rayleigh and Raman processes . . . . . . . . . . . . . . . . . . 155

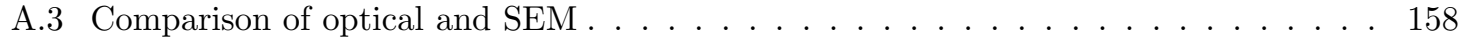

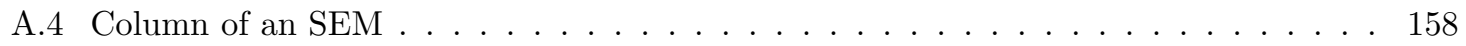

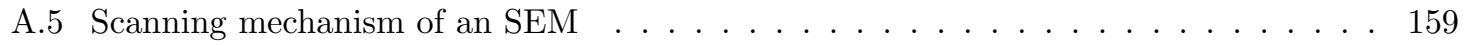




\section{List of Tables}

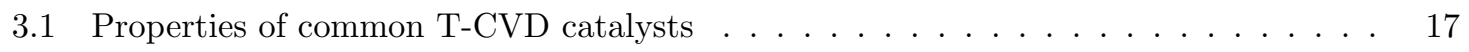

3.2 Stock TCVD MWCNTs growth conditions . . . . . . . . . . . . . . . 25

3.3 Temperature and pretreatment gas results for bamboo MWCNTs . . . . . . . . . . 28

3.4 Ideal bamboo conditions . . . . . . . . . . . . . . . . . . . . . . . . . . . 32

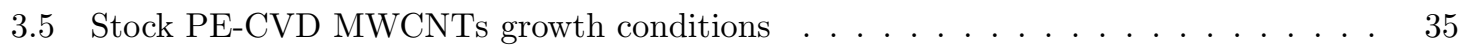

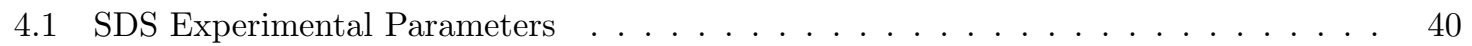

6.1 Comparison of different novel RAM technologies. . . . . . . . . . . . . . . . . 92

7.1 Summary of STM measurement parameters . . . . . . . . . . . . . . 117

7.2 Roughness comparison of metal film deposition methods. . . . . . . . . . . . . . 126

7.3 Roughness comparison of annealed $\mathrm{Cr} / \mathrm{Au}$ films. . . . . . . . . . . . . . . 127

7.4 Roughness of $60 \mathrm{~nm} \mathrm{Au} \mathrm{films} \mathrm{\ldots ...} \mathrm{.} \mathrm{.} \mathrm{.} \mathrm{.} \mathrm{.} \mathrm{.} \mathrm{.} \mathrm{.} \mathrm{.} \mathrm{.} \mathrm{.} \mathrm{.} \mathrm{.} 130$ 


\section{Units and Abbreviations}

\begin{tabular}{|c|c|}
\hline $2 \mathrm{D}$ & Two Dimensional \\
\hline $3 \mathrm{D}$ & Three Dimensional \\
\hline $\mathrm{AC}$ & Alternating Current \\
\hline $\mathrm{Ag} \mid \mathrm{Ag}^{+}$ & Silver|Silver Ion Quasi-Reference Electrode \\
\hline AFM & Atomic Force Microscope \\
\hline $\mathrm{cm}^{-1}$ & Inverse Wavelengths, Standard Units for Raman Shift \\
\hline $\mathrm{CM}$ & Clausius-Mossotti \\
\hline $\mathrm{CNT}$ & Carbon Nanotube \\
\hline CVD & Chemical Vapor Deposition \\
\hline $\mathrm{DC}$ & Direct Current \\
\hline DEP & Dielectrophoresis \\
\hline FE-SEM & Field Emission Scanning Electron Microscope \\
\hline FIB & Focused Ion Beam \\
\hline FTIR & Fourier Transform Infrared Spectrometer \\
\hline $\mathrm{G} / \mathrm{D}$ & Graphitic Peak Intensity to Disorder Peak Intensity Ratio \\
\hline $\mathrm{HOPG}$ & Highly Organized Pyrolytic Graphite \\
\hline IPA & Isopropyl Alcohol \\
\hline IR & Infrared \\
\hline MWCNT & Multi-Walled Carbon Nanotubes \\
\hline PECVD & Plasma-Enhanced Chemical Vapor Deposition \\
\hline PLD & Pulsed Laser Deposition \\
\hline PPF & Pyrolized Photoresist Film \\
\hline $\mathrm{RBM}$ & Radial Breathing Mode \\
\hline $\operatorname{sccm}$ & standard cubic centimeter per minute \\
\hline SEM & Scanning Electron Microscope \\
\hline SWCNT & Single-Walled Carbon Nanotube \\
\hline TCVD & Thermal Chemical Vapor Deposition \\
\hline TBABF4 & Tetrabutyl Ammonium Tetrafluoroborate \\
\hline TEM & Transmission Electron Microscope \\
\hline VLS & Vapor-Liquid-Solid \\
\hline YAG & Yttrium Aluminum Garnet \\
\hline
\end{tabular}




\section{Chapter 1}

\section{Research Overview}

\subsection{Background and Motivation}

In the first half of the 20th century, the field of electronics began in earnest with the spread of communication technologies and the advancement of radar [1]. The first transistor, invented in 1947 by John Bardeen and Walter Brattain at Bell Labs, was a point-contact resistor composed of a germanium substrate in contact with three gold wires [1]. Next to come, in 1948 by William Shockley, was the bipolar transistor consisting of alternating layers of p- and n-type germanium [1]. In 1954, germanium was replaced with the now ever-present silicon by Texas Instruments. The basis of most electronics, the field-effect transistors (FETs), were introduced in 1961, and not much has changed since...until recently $[1,2]$.

The one thing that has consistently changed is size. In order to keep the number of transistors per $\mathrm{cm}^{2}$ doubling roughly every two years, the transistors have to keep shrinking $[1,2]$. However, in order to halve the size of the transistors, the smallest parts of the FETs have to be diminished by a factor of 0.7 [2]. Clearly, this size reduction cannot continue forever before fundamental limits are reached. But it is precisely this size reduction that has been fueling the progress explosion driving electronics technologies for the past 60 years.

Few things define the modern world as much as electronics. From the personal to medical, military and scientific equipment, electronics have allowed progress in ways unimagined before their advent. But our world of ever-improving electronics has relied on the shrinking of transistors as 
the basis of increases in computational power. If we want this trend to continue, the manufacturing processes are going to have to change.

For example, Intel, the world leader in complementary metal oxide semiconductor (CMOS) FETs, has had to move away from silicon oxide dielectrics and silicon gates in order to achieve their current $45 \mathrm{~nm}$ transistors [2]. For an industry based around silicon technology to move away from silicon after so long shows how serious change is needed. Intel is pushing the limits by moving away from traditional materials, while staying with familiar architectures. But there are other approaches.

\section{Molecular Electronics}

One such approach is to make devices as small as we can: out of molecules. It has already been demonstrated that molecules forming junctions (between electrodes) can show a wide variety of electronic behavior from conductance switching $[3,4,5]$ to rectification $[6,7]$ and negative differential resistance [8]. Therefore, certain molecules have the potential to be used directly as components within electronic circuits. Devices based on these ideas have been realized to various degrees, even up to molecular memory with a density of $10^{11}$ bits per square centimeter [9]. Yet while such devices are being constructed, there is still a lot of effort being put into making them work. Small working yields and low switching limits before burning out are some of the problems that must be addressed before achieving viability and, unfortunately, the failure mechanisms and electronic transport properties are not properly understood $[4,10]$.

\subsection{Our Approach}

Carbon nanotubes (CNTs), nano-scale cylinders of graphitic carbon first discovered in 1991 [11], were found to have unique structural and electronic properties such as superior mechanical strength, high electrical and thermal conductivity and high chemical stability [12, 13]. While CNTs with single walls can be either semiconducting or semi-metallic depending on their chirality, multi-walled CNTs (MWCNTs, groups of concentric cylinders) are always semi-metallic, making them perfect candidates for nanoscale wires and interconnects. Already, CNTs have been integrated into nanoelectronic devices such as field effect transistors and single electron transistors $[14,15,16,17,18]$. 


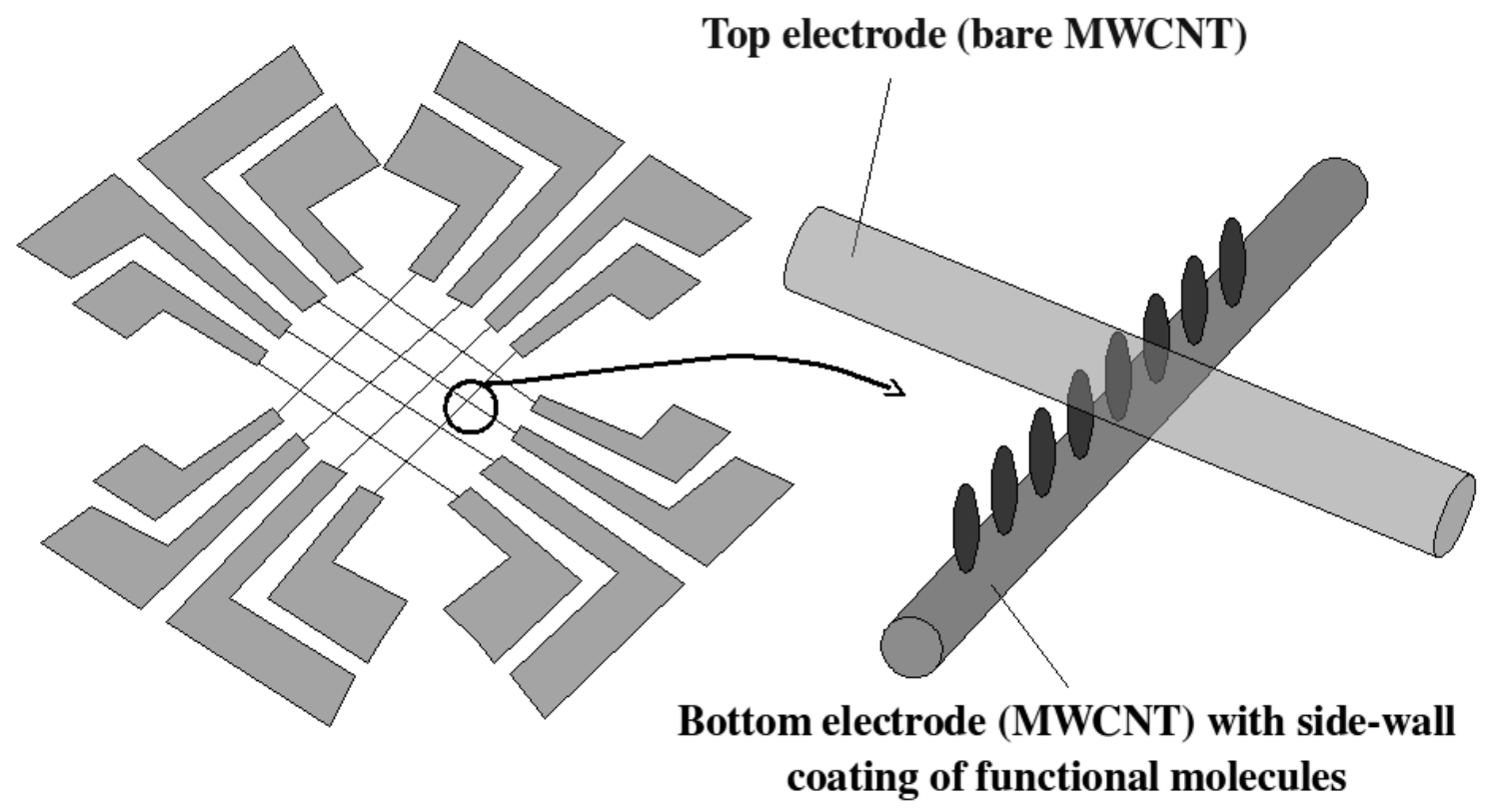

Figure 1.1: Schematic representation of the original molecular electronics device design. Carbon nanotubes connect electrode pads, forming arrays of crosses. The nanotubes in one direction have functionalized sidewalls, so that each CNT cross is a junction containing an organic molecule.

\section{Molecular Junction}

Out of all the nanomaterials being studied, CNTs have one unique strength that others do not: they are wholly carbon. When covalently functionalized with organic molecules, bonds to the molecule form via strong carbon-carbon bonds. It is our contention that the strength of these bonds will extend the lifetime of the organic molecular junctions. To that end, our main goal was to create a process of fabricating MWCNT-organic molecule-MWCNT junctions. Once we could make such junctions, we could freely swap out the molecule to ones specifically tailored to act as circuit components and test their effectiveness in future work. Our initial concept for the junction device is shown in Figure 1.1.

\section{Sensors}

The processes and techniques needed to reach our goal lead us naturally to test and build other devices, namely chemical and biological sensors. MWCNT's tubular structure means most atoms are located on or near the surface. Therefore any surface interactions are interacting with the bulk 
of the material, making them extremely sensitive to surface changes and leading to the ability to detect very low concentrations. With a wide variety of options for functionalizing the MWCNTs, we can create sensors of many different types such as DNA, glucose or gas sensors. Further, the small dimensions and high sensitivity of CNT based sensors make them perfect for "lab on a chip" applications where a variety of substances are tested for simultaneously, which has extremely high value in the military and medical fields. I will discuss my work on MWCNT-based glucose sensors.

\subsection{Dissertation Work}

My dissertation work was the first full project to take the group from its origins of nanomaterial growth through modification, placement and, ultimately, device construction - all done in-house. This meant building up group resources and techniques that we didn't yet have, including Raman and FTIR spectroscopy, scanning probe microscopy (AFM and STM), photolithography (choice of resists, design of masks, evolution of techniques) and electrochemistry (laboratory design, glove-box set-up, safety documentation and procedures). As such, my work touches on a wide variety of topics.

The flow of the research is shown in Figure 1.2. In order to make the final junctions, it was necessary to master MWCNT growth, (electro)chemical methods, photolithography/thin films and dielectrophoresis. The etching of MWCNTs was found to have a positive effect on their field emission, and could also be used to functionalize them to make devices such as glucose sensors.

The discussion of these topics is broken up as follows:

- Chapter 2 introduces the necessary background on carbon nanotubes.

- Chapter 3 discusses the growth methods used in my research, and details my first project, finding the proper conditions for growing bamboo-like multi-walled carbon nanotubes.

- Chapter 4 focuses on placement of carbon nanotubes, both by surfactant-assisted dispersion and surfactant-free dielectrophoretic deposition.

- Chapter 5 covers the acid etching of carbon nanotubes and some of the experiments that have come out of the investigation. 


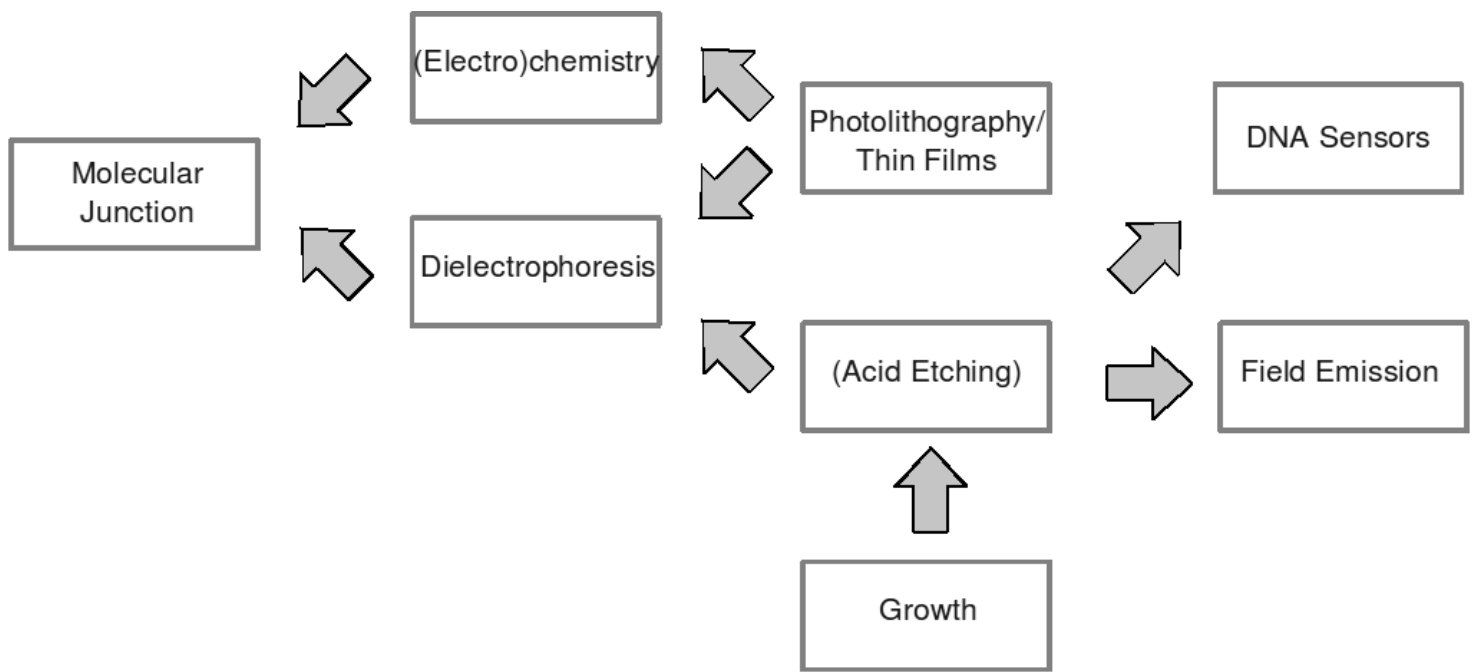

Figure 1.2: A flowchart showing the basic relationships between the investigated processes and goals.

- Chapter 6 discusses the initial design of the molecular junction, the problems we discovered over the course of the research, and the changes we made to accommodate them. Our work on the carbon-molecule-carbon is discussed.

- Chapter 7 involves the fabrication and testing of the gold-molecule-carbon junctions.

- Appendix A serves to explain a few techniques that are commonly used and central to this dissertation work for the reader and future researchers. 


\section{Chapter 2}

\section{Introduction to Carbon Nanotubes}

Carbon nanotubes (CNTs) are cylinders of graphitic phase carbon discovered in 1991 [11]. Massive amounts of research have gone into studying and using CNTs because they have impressive properties including superior mechanical strength, high electrical and thermal conductivity and high chemical stability[12, 13]. These characteristics have facilitated their integration into a wide array of devices and applications such as field effect transistors [14, 15, 16, 17, 18], chemical and biological sensors $[19,20]$, scanning probe microscopy tips [21, 22, 23], field emitters [24], interconnects [25, 26], in vivo drug delivery vectors [27], nanoelectrodes [28, 29] and more.

Given the innumerable topics available, it is impossible to cover them all. Therefore, this chapter serves only as an introduction to CNTs, with information relevant to the work done in this dissertation.

\subsection{Carbon and Bonding}

Carbon is a very flexible element. It can form open-chain or ring compounds by covalently bonding to other carbon in single, double or triple bonds [30]. Looking at free carbon atoms, we see the electronic structure is $1 s^{2} 2 s^{2} 2 p^{2}$, one closed shell and four valence electrons; it is the valence electrons that enable covalent bonding. In carbon, the energy difference between the $s$ and $p$ orbitals is small compared to the energy of the chemical bonds, so the orbitals can mix with each other in order to enhance the binding energy with its neighbors [31]. In general, one $2 s$ electron is promoted to $2 p$ 
so that the structure is more akin to $1 s^{2} 2 s^{1} 2 p^{3}$ and the orbitals hybridize in one of three ways to form different materials [13]. The different bonding types lead to different phases or allotrope's of carbon.

The first such hybridization is called $s p$ bonding. In this case, the $2 s$ orbital combines with one of the two $2 p$ orbitals (Figure 2.1). If we take a hybridization with the $2 \mathrm{p}_{x}$ as an example, the resulting hybrid orbitals can be represented by[31]:

$$
\begin{aligned}
& \left|s p_{a}\right\rangle=\frac{1}{\sqrt{2}}\left(|2 s\rangle+\left|2 p_{x}\right\rangle\right) \\
& \left|s p_{b}\right\rangle=\frac{1}{\sqrt{2}}\left(|2 s\rangle-\left|2 p_{x}\right\rangle\right)
\end{aligned}
$$

Only when a highly asymmetric bond is favourable will $s p$ bonding take place, so it does not occur in the crystalline allotropes of carbon. An example can be found in bonding structure of the hydrocarbon acetylene: $\mathrm{HC} \equiv \mathrm{CH}$. Here, the $\left|s p_{a}\right\rangle$ orbital of one carbon bonds covalently to the $\left|s p_{b}\right\rangle$ of the other; head-to-head overlap of this type is called a $\sigma$ bond [30,31]. The other two bonds that help constitute the triple bond are the parallel $p_{y}$ and $p_{z}$ orbitals of each carbon atom; side-by-side overlap is called $\pi$ bonding [30,31]. Single bonds are always $\sigma$, while doubles are one $\sigma$ and one $\pi$, and triples are one $\sigma$ and two $\pi[30]$.

In $s p^{2}$ hybridization, the $2 s$ orbital and two $2 p$ orbitals mix (Figure 2.2). If we choose the $\mathrm{x}$ and y direction for hybridization, the orbitals are represented by [31]:

$$
\begin{aligned}
& \left|s p_{a}^{2}\right\rangle=\frac{1}{\sqrt{3}}|2 s\rangle-\frac{2}{\sqrt{3}}\left|2 p_{y}\right\rangle \\
& \left|s p_{b}^{2}\right\rangle=\frac{1}{\sqrt{3}}|2 s\rangle+\frac{1}{\sqrt{2}}\left|2 p_{x}\right\rangle+\frac{1}{\sqrt{6}}\left|2 p_{y}\right\rangle \\
& \left|s p_{c}^{2}\right\rangle=-\frac{1}{\sqrt{3}}|2 s\rangle-\frac{1}{\sqrt{2}}\left|2 p_{x}\right\rangle+\frac{1}{\sqrt{6}}\left|2 p_{y}\right\rangle
\end{aligned}
$$

The $s p^{2}$ orbitals have large amplitude in the direction of the nearest carbon atoms, are all within a single plane and are separated in direction by $120^{\circ}$. The remaining $p_{z}$ orbitals are directed perpendicularly out of the plane of the hybridized bonds [32]. Planar sheets of all-carbon bonded solely by $s p^{2}$ orbitals are referred to as graphene, with layers of such sheets forming the familiar 

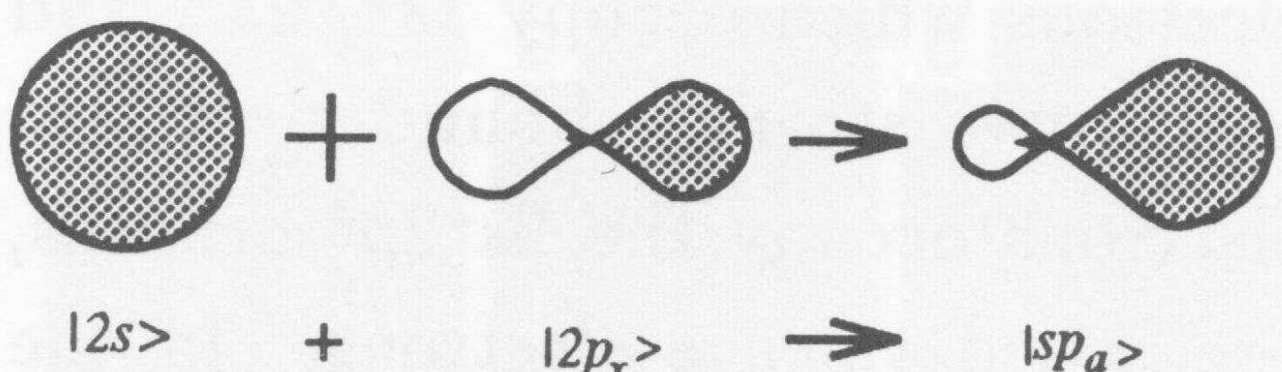

$12 p_{x}>$

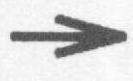

$\left|s p_{a}\right\rangle$
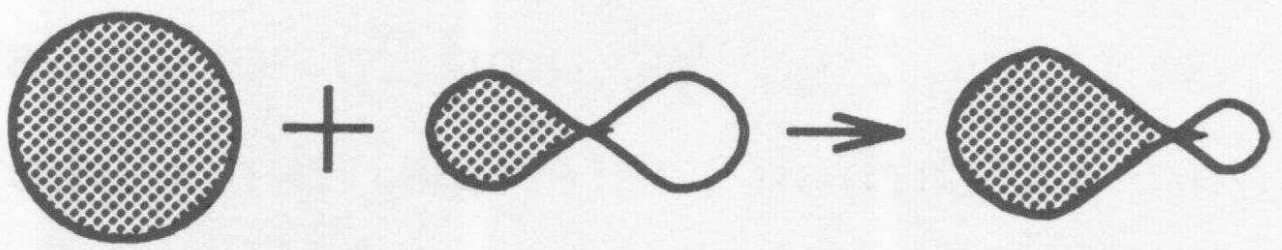

$12 s\rangle$
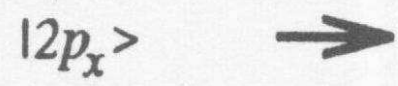

$\left|s p_{b}\right\rangle$

Figure 2.1: A simplified visual representation of the highly asymmetric orbitals formed by $s p$ hybridization. Figure reprinted with permission from Imperial College Press in reference [31].

graphite (Figure 2.3). In graphite, the $s p^{2}$ orbitals form strong $\sigma$ bonds between atoms in the same graphene layer while the remaining $\pi$ orbitals are responsible for the weak van der Waals bonding between different graphite planes [13]. It is the stacking of the $\pi$ bonds that provide pathways for electrons between graphene layers and helps account for the high conductivity of graphite [13]. Graphene sheets stack in what is known as ABAB stacking, or Bernal stacking, where the alternate planes are shifted relative to one another [13].

The last hybridization is a mixing of the $2 s$ orbital and all three $2 p$ orbitals. The resulting orbitals are in the following directions [31]: 


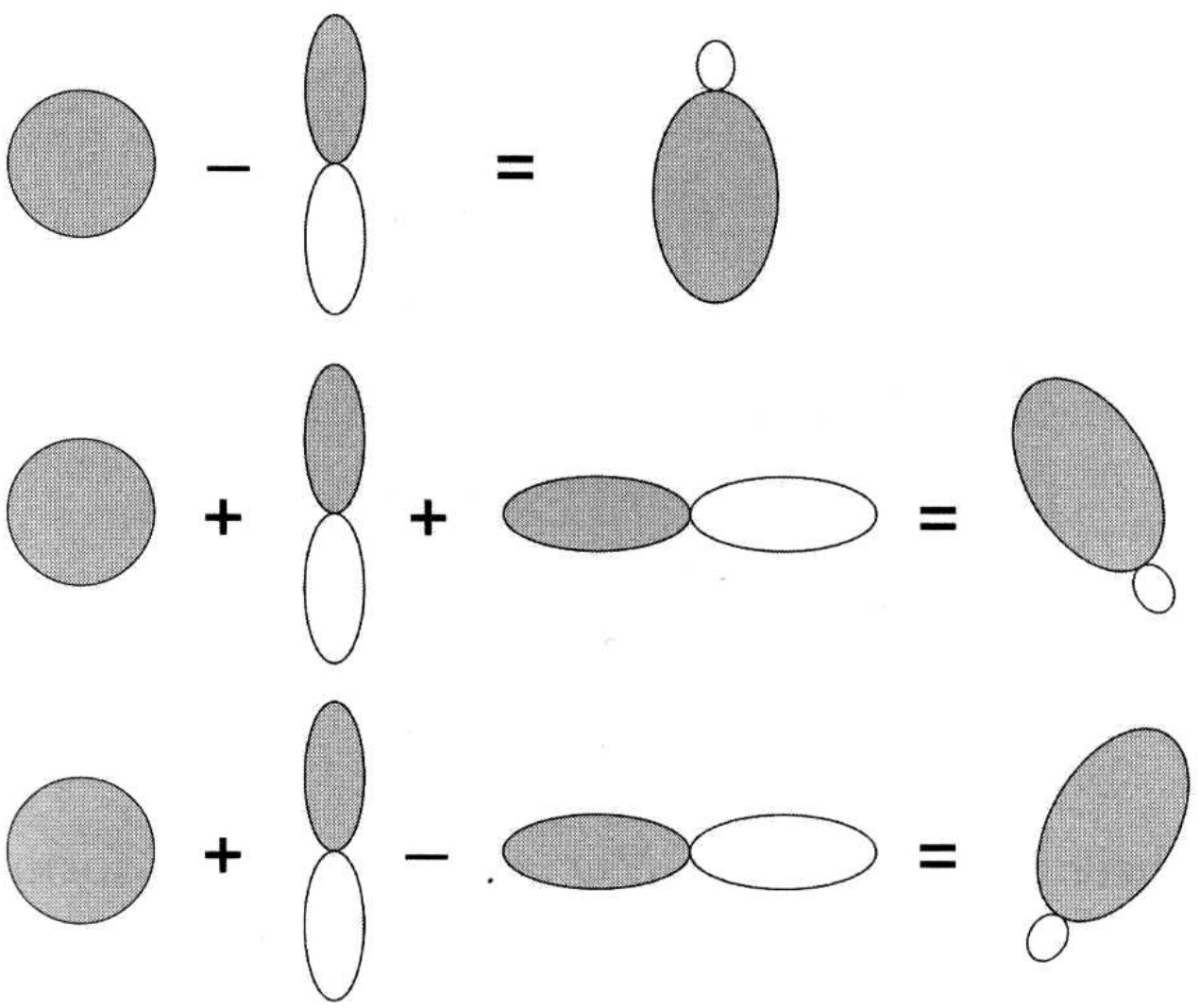

Figure 2.2: A visual representation of the orbitals formed by $s p^{2}$ bonding. Figure reprinted with permission from Elsevier from reference [32]. 


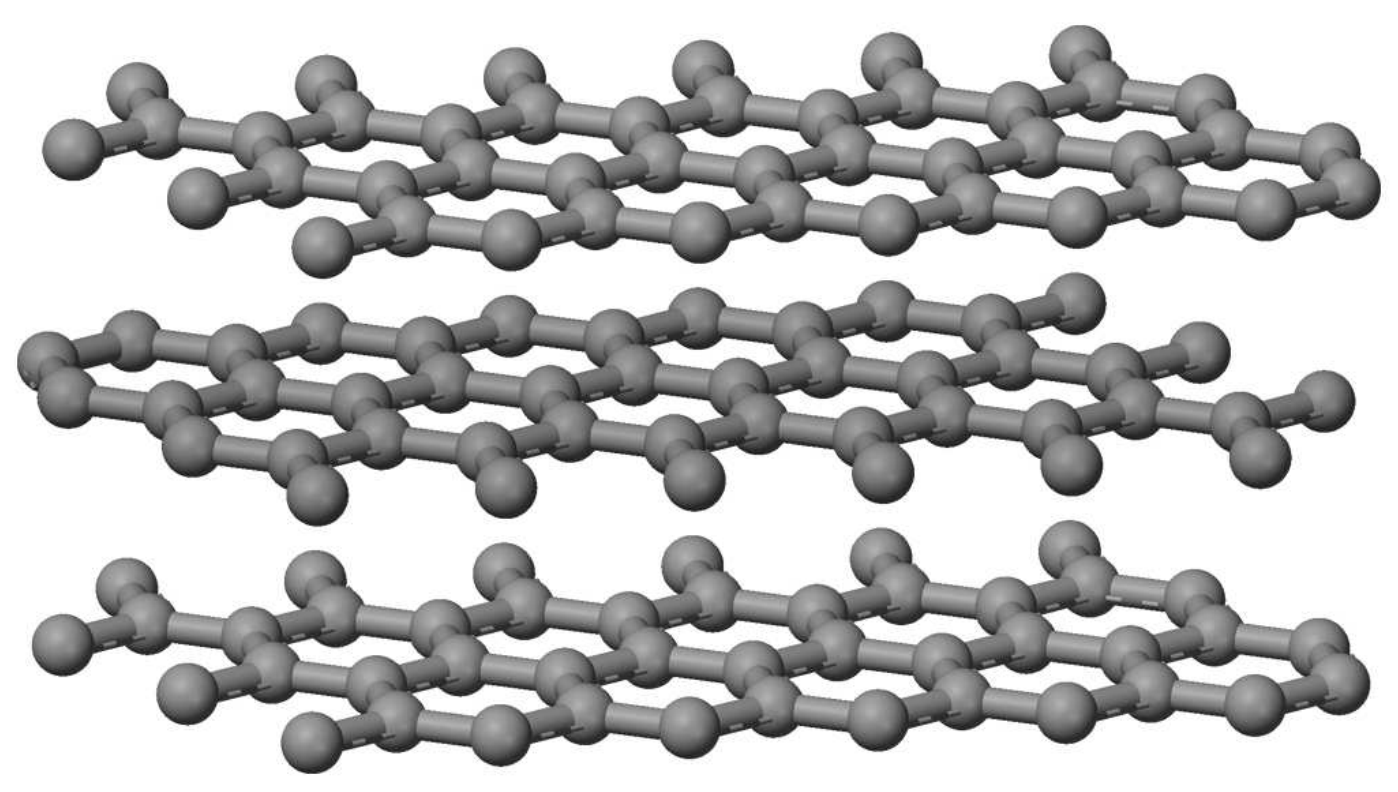

Figure 2.3: Planes of hexagonally-arranged carbon molecules form planar graphene sheets, while the sheets stack to form graphite.

$$
\begin{aligned}
\left|s p_{a}^{3}\right\rangle & =\frac{1}{2}\left(|2 s\rangle+\left|2 p_{x}\right\rangle+\left|2 p_{y}\right\rangle+\left|2 p_{z}\right\rangle\right) \\
\left|s p_{b}^{3}\right\rangle & =\frac{1}{2}\left(|2 s\rangle-\left|2 p_{x}\right\rangle-\left|2 p_{y}\right\rangle+\left|2 p_{z}\right\rangle\right) \\
\left|s p_{c}^{3}\right\rangle & =\frac{1}{2}\left(|2 s\rangle-\left|2 p_{x}\right\rangle+\left|2 p_{y}\right\rangle-\left|2 p_{z}\right\rangle\right) \\
\left|s p_{d}^{3}\right\rangle & =\frac{1}{2}\left(|2 s\rangle+\left|2 p_{x}\right\rangle-\left|2 p_{y}\right\rangle-\left|2 p_{z}\right\rangle\right)
\end{aligned}
$$

In this case, all bonds formed will be $\sigma$ bonds, three of the bonding directions are free, but the final direction must satisfy the orthonormality condition imposed by the $p$ orbitals [31]. Diamond is composed of $s p^{3}$ bonded carbon (Figure 2.4). Diamond, though incredibly hard, is a less chemically stable structure than graphite, and will convert to graphite at $1700^{\circ} \mathrm{C}$ at one atmosphere [13].

It is here we see the extreme importance of bonding to material properties. For example, even though both graphite and diamond are composed solely of carbon, the different hybridization leads to 


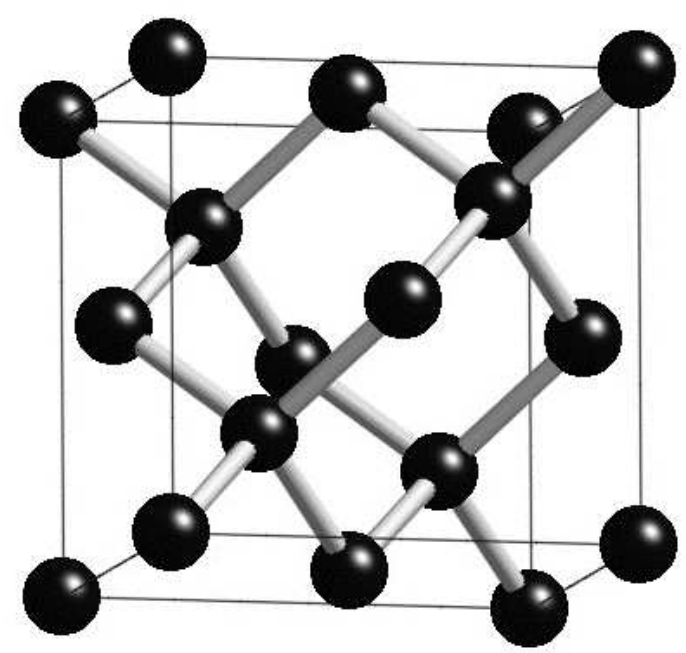

Figure 2.4: Diamond is an allotrope of carbon composed of $s p^{3}$ hybridized orbitals. Unlike the other hybridizations, the orbitals cannot all lie in the same plane leading to more complex geometries. Figure used with permission from reference [33].

vastly different properties: graphite is soft, conductive and opaque while diamond is hard, insulating and transparent. The extreme effect of bond and orbital type will be important later when discussing crystalline defects and bonding other molecules to the CNTs.

\subsection{Carbon Nanotubes Discovery}

Harry Kroto from the University of Sussex and Richard Smalley of Rice University, Houston, were analyzing carbon clusters formed by vaporization of carbon. In a series of experiments starting in 1985 they discovered that in their gas-phase carbon clusters, $\mathrm{C}_{60}$ was the dominant species. $\mathrm{The}_{60}$ molecules, called buckminster fullerenes, had exactly 60 carbon atoms, forming a closed structure [13]. Later, Wolfgang Krätschmer of the Max Planck Institute, Heidelberg, Donald Huffman of the University of Arizona and their coworkers discovered a simpler carbon arc technique for producing macroscopic quantities of fullerenes. This discovery set off an avalanche of research into carbon structures [13].

Carbon nanotubes (CNTs), another special geometry of carbon, had been speculated on by Richard Smalley [31] and experimentally observed in 1991 by Sumio Iijima of NEC Research Labo- 
ratory in Japan [11]. Iijima had been examining the synthesis product of an arc-discharge experiment under a high-resolution transmission electron microscope and accidentally discovered the presence of multi-walled carbon nanotubes (MWCNTs) in the carbon soot of the graphite electrodes [11]. Two years later, Iijima discovered single-walled carbon nanotubes (SWCNTs) [34].

\subsection{Carbon Nanotubes Structures}

Put simply, carbon nanotubes are cylinders of graphitic $\left(s p^{2}\right)$ carbon. To conceptualize a singlewalled carbon nanotube, it is easy to think of a single graphene sheet that is rolled up to form a seamless cylinder. Different ways of rolling up the sheet result in tubes of different chiralities. The conventional way of describing the structure is through the use of a vector $\vec{C}$ which joins two points on the lattice. The cylinder is formed by rolling the sheet such that these two points become superimposed [13, 31, 32].

The vector, shown in Figure 2.5, can be written as:

$$
\vec{C}=n \overrightarrow{a_{1}}+m \overrightarrow{a_{2}}
$$

where $n$ and $m$ are integers and $n \geq m$. For all tubes in which $m=0$, the tubes are said to be zig-zag. For the case of $\mathrm{n}=\mathrm{m}$, they are armchair. For any other values, the tubes are said to be chiral [13]. A visual representation of the "rolling" and chiralities is shown in Figure 2.5. Geometrically from the lattice it follows that $\left|\overrightarrow{a_{1}}\right|=\left|\overrightarrow{a_{2}}\right|=0.246 \mathrm{~nm}$. Therefore, the diameter of a SWCNT can be expressed as $[13,31,32]$ :

$$
d=0.246 \frac{\sqrt{n^{2}+n m+m^{2}}}{\pi}
$$

with the chiral angle is represented as [13]:

$$
\theta=\sin ^{-1} \frac{\sqrt{3 m}}{2 \sqrt{n^{2}+n m+m^{2}}}
$$

Electronically, SWCNTs are either semi-conductors or metallic based on chirality. Armchair SWCNTs are metallic as are all chiral nanotubes that satisfy the condition $n-m=3 q$ where $q$ is an integer; all other SWCNTs are semi-conductors $[13,32]$. However, as the CNT diameter increases, 
the band gap decreases until the tubes become metallic [32]:

$$
E_{\text {gap }} \approx \frac{2 \times 0.142 \mathrm{~nm} \times \gamma}{d}
$$

where $\gamma$ is the tight-binding parameter that represents the strength of nearest-neighbor interactions and $d$ is the radius of the semiconducting SWCNT. This is shown for zig-zag SWCNTs in Figure 2.6 .

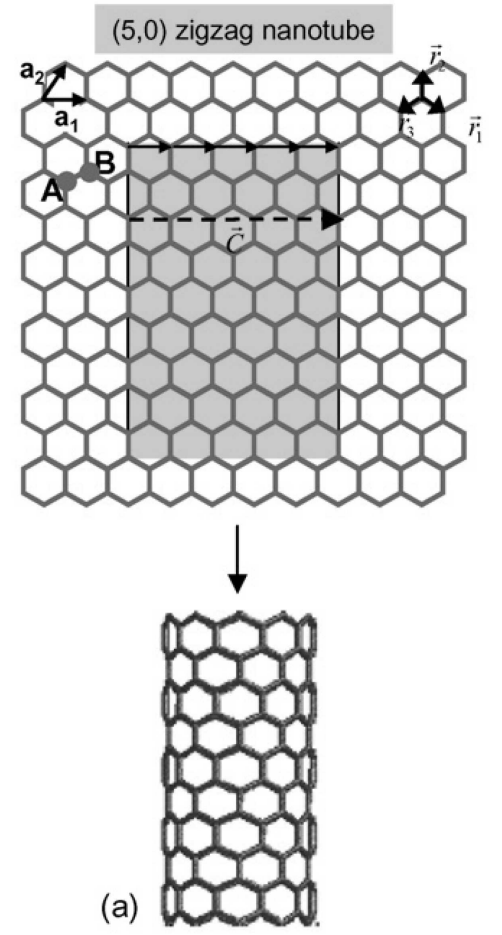

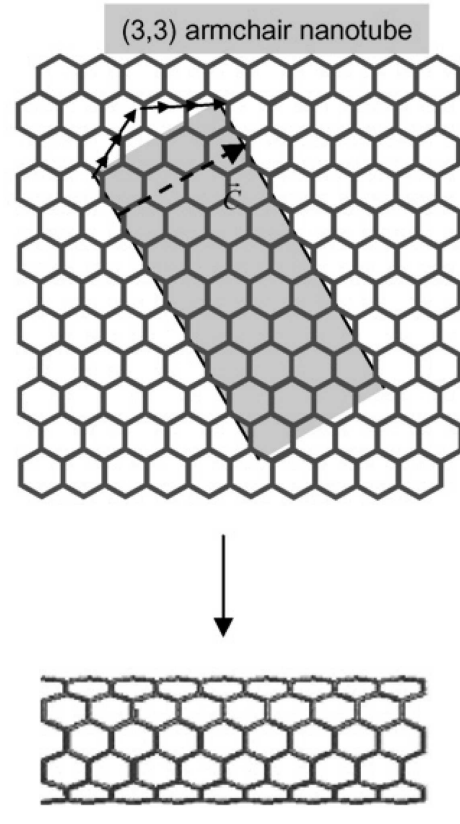

(b) (c)
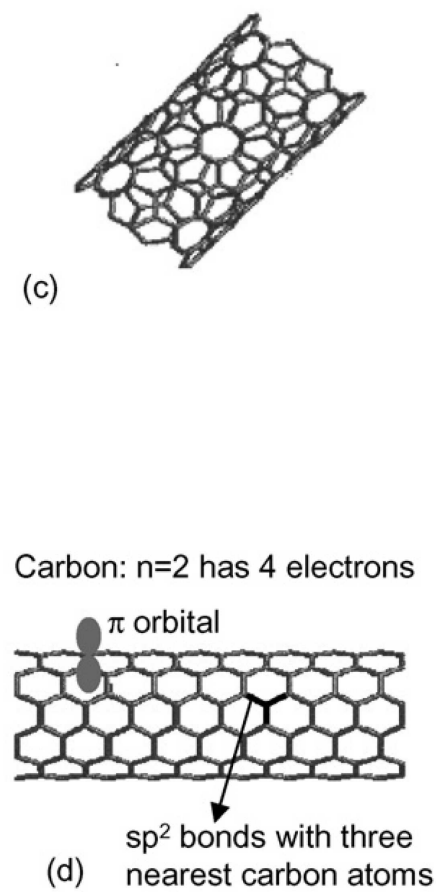

Figure 2.5: Folding graphene sheets to form CNTs. Figure reprinted with permission from Elsevier in reference [32].

It must be mentioned that when growing SWCNTs, it is impossible to control the chirality they grow with a distribution of chiralities [31]. Therefore, if a specific band-gap or conductance is desired, a selection process must be used post-growth in order to isolate the wanted species from the unwanted.

As well as having tubes with single walls it is possible to have concentric geometries, with many walls for a single tube. These structures are called multi-walled carbon nanotubes (MWCNTs). Due 


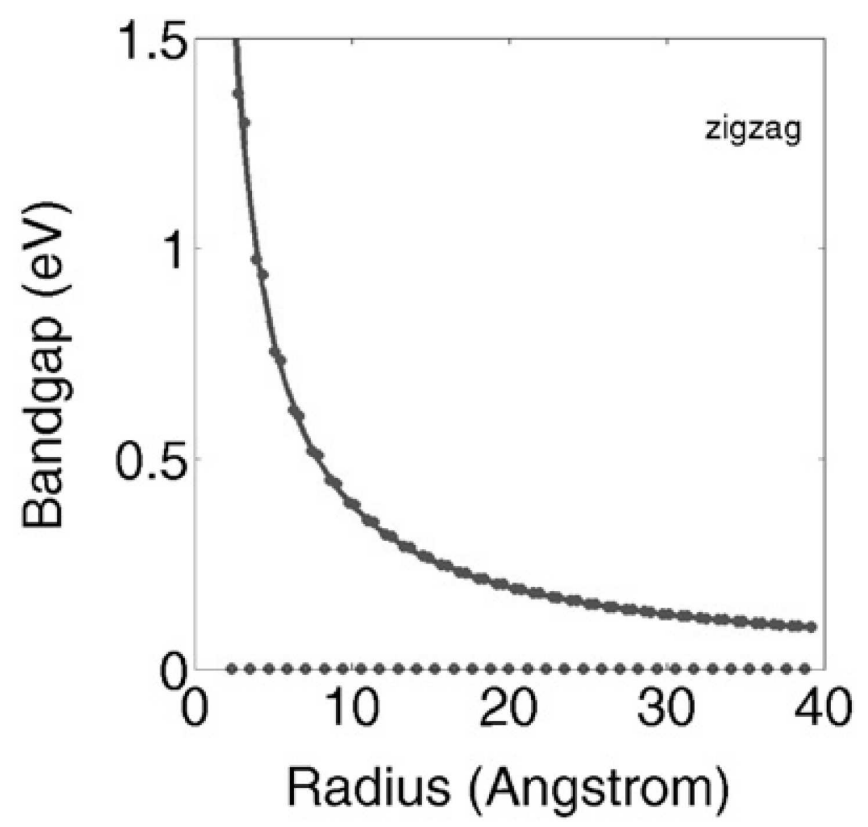

Figure 2.6: The bandgap of SWCNTs shrinks with increasing tube diameter. The curve shown here is for zig-zag SWCNTS. Figure used with permission from Institute of Physics from reference [35].

to the large diameters and high number of walls, generally all MWCNTs with more than two walls are metallic [32]. Due to the shape of the MWCNTs, it is impossible that the inter-wall spacing be the same of graphite, $0.334 \mathrm{~nm}$ [13]. For example, the closest to the graphite spacing two armchair walls can come is $0.352 \mathrm{~nm}$. The walls attempt to preserve this spacing as well as the ABAB stacking, but the curved shape makes it impossible to exactly achieve either [13]. Experimental measurements show that the inter-layer spacing can vary a lot, especially since there is no requirement that all walls have the same chirality. However, generally speaking, the spacing is on the order of $0.34 \mathrm{~nm}$ [13].

\subsection{Carbon Nanotubes Growth}

There are two main categories of CNT growth techniques, discussed below. 


\section{Sublimation/Desublimation of Graphite and Arc Discharge}

The first category uses graphite as a carbon source and extremely high (up to $4000{ }^{\circ} \mathrm{C}$ ) temperatures in order to make the graphite sublimate, then desublimate elsewhere. Possibilities include laser ablative, focused radiation, resistive heating and arc discharge techniques. As a popular and historically important method, arc discharge will be explained as an example of this approach [36].

Typically, carbon rod electrodes are separated by $\sim 1 \mathrm{~mm}$ with a voltage of $20-25 \mathrm{~V}$ between them and about 50-120 A flowing. The environment is roughly 500 Torr He with a small flow rate of $5-15 \mathrm{~mL} / \mathrm{s}$ for cooling. The length of the positive electrode decreases as carbon deposits on the negative electrode. Nanotube bundles are found in the inner region of the deposit where the temperature reaches its maximum of $2500-3000{ }^{\circ} \mathrm{C}$. The nanotubes are surrounded by a shell of nanoparticles, fullerenes and amorphous carbon, so a purification step is necessary in order to retrieve the tubes $[31,36]$. Another major drawback to this method is it is extremely difficult to make a continuous process, so while the CNTs produced are very pristine, their length is limited [36].

\section{Decomposition and Chemical Vapor Deposition}

The second category uses decomposition of a carbon source, be it gas (chemical vapor deposition), solid or an aqueous (hydrothermal synthesis) or organic solution [36]. Chemical vapor deposition (CVD) is a large category of related decomposition methods that take place at low temperatures of $500-1300{ }^{\circ} \mathrm{C}$, so it is more compatible for on-device growth, and can be continuous in growth, leading to extremely long tubes $[31,36]$. In CVD, a hydrocarbon gas is introduced and decomposed by temperature or plasma to create the carbon source. The growth methods employed in this dissertation were CVD methods and will be discussed in more detail in the following chapter. 


\section{Chapter 3}

\section{Carbon Nanotube Growth}

Growth of carbon nanotubes comprised the first projects in my dissertation research. First, I assisted in optimization of the growth conditions of MWCNT growth after furnace system modification, learning the growth methods. Second, working with the assistance of Vijaya Kayastha, I investigated under what growth conditions we could grow bamboo-like MWCNTs. Third, growth of MWCNTs by both thermal and plasma-enhanced chemical vapor depositions were carried out to produce the stock of MWCNTs I used during my greater dissertation project. Last, I contributed growth of these stock tubes for use in the field emission experiments conducted by Ben Ulmen and Vijaya Kayastha. In this chapter I will describe the growth methods and the carbon nanotubes produced by them.

\subsection{Thermal Chemical Vapor Deposition Process}

From start to finish, CVD growth of MWCNTs consists of three major steps:

(1) Deposition of the catalyst film

(2) Pretreatment

(3) Growth

\subsubsection{Deposition of the Catalyst Film}

Transition metals are generally used as catalysts for growing CNTs with $\mathrm{Fe}$, Ni, Co and Mo as popular choices $[13,31]$. The metal nanoparticles that will form from the film during step two 
Table 3.1: Select properties of common catalyst metals

\begin{tabular}{|l|l|l|l|l|}
\hline Bulk Property & Fe & Ni & Co & Mo \\
\hline \hline $\begin{array}{l}\text { Density at } 20^{\circ} \mathrm{C} \text { (liquid state) } \\
\left(\frac{\mathrm{kg}}{\mathrm{m}^{3}}\right)[37]\end{array}$ & $7874(7035)$ & $8902(7780)$ & $8900(7670)$ & $10220(9330)$ \\
\hline Melting temperature $\left({ }^{\circ} \mathrm{C}\right)$ & 1536 & 1453 & 1495 & 2617 \\
\hline $\begin{array}{l}\text { Carbon solubility }(\text { at. \%) at } \\
\text { melting temperature }[38]\end{array}$ & 20.2 & 10.7 & 13.9 & Up to 60-70 \\
\hline $\begin{array}{l}\text { Carbon diffusion coefficient } \\
\text { at } 1000{ }^{\circ} \mathrm{C}\left(\frac{\mathrm{m}^{2}}{\mathrm{~s}}\right)[39]\end{array}$ & $1.5 \times 10^{-11}$ & $2 \times 10^{-11}$ & $1 \times 10^{-11}$ & $\mathrm{~N} / \mathrm{A}$ \\
\hline $\begin{array}{l}\text { Equilibrium vapor pressure } \\
\text { at } 1216^{\circ} \mathrm{C}(\mathrm{Pa})[40]\end{array}$ & $5.5 \times 10^{-3}$ & $4 \times 10^{-3}$ & $4.7 \times 10^{-3}$ & $7.6 \times 10^{-11}$ \\
\hline $\begin{array}{l}\text { Surface tension } \\
\text { at } 20(2000){ }^{\circ} \mathrm{C}\left(\frac{\mathrm{N}}{\mathrm{m}}\right)[41]\end{array}$ & $1.95(1.72)$ & $1.86(1.57)$ & $2.1(1.68)$ & $2.2(\mathrm{~N} / \mathrm{A})$ \\
\hline
\end{tabular}

provide nucleation sites for growth and also assist in the carbon source decomposition. Choosing an appropriate catalyst is important and different optimizations of other growth parameters will be necessary for each choice. The desired characteristics are the ability to catalytically decompose the hydrocarbons and their carbon solubility. Table 3.1 lists some properties of common catalysts, showing how, with some exceptions for Mo, there is little variation.

Catalysts should also have good metal-support interaction, high surface area, large pore volume and, importantly, these properties must remain intact at the high temperatures necessary for growth [12]. A strong metal-support interaction allows the catalyst particles that form to remain dispersed with a high density of sites and prevents aggregation into large clusters. Large surface area means more catalytic sites and surface area for carbon diffusion.

Catalyst films are prepared by a variety of different techniques including the precipitation of metal salts (nitrates, sulfates, etc.), sputtering [42], laser deposition [43, 44] and liquid chemistry routes such as organic carriers [45, 46, 47]. For my thermal CVD growth experiments, all films were deposited on various types of silicon substrates by what is called the pulsed laser deposition (PLD) method.

The roots of pulsed laser deposition began when the first high-powered ruby lasers became available which in turn lead to massive study on the interactions of the laser beams with solid surfaces, liquids and gaseous materials. Since the highly intense laser beams vaporize the material readily, it was predicted that this phenomenon could be used to deposit thin films, and a few 


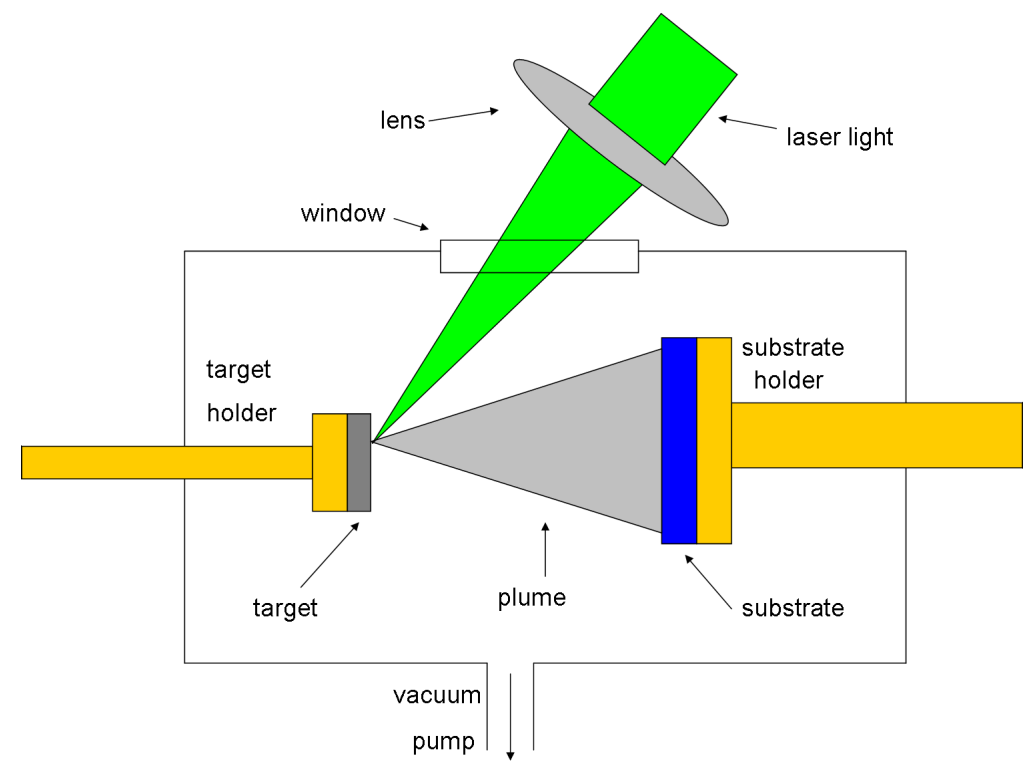

Figure 3.1: A schematic showing the pulsed laser deposition process. The substrates are mounted on a holder and mounted to face the metal target inside the vacuum. A laser fires pulses which vaporize the target and sends a plume of material toward the substrate causing it to deposit evenly.

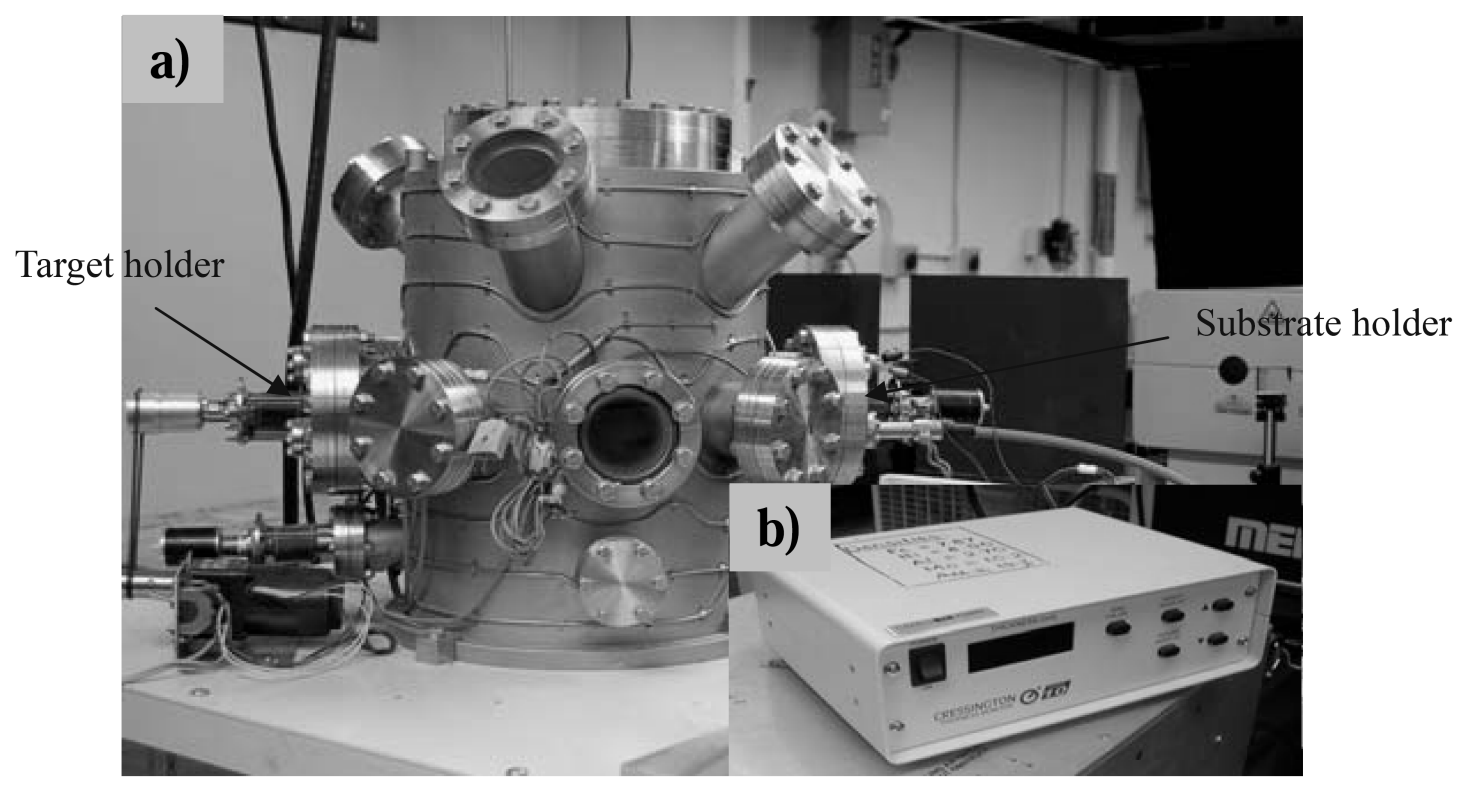

Figure 3.2: (a) The PLD vacuum chamber. (b) The display and controls for the thickness monitor. Figure reprinted with permission from reference [48]. 
years later, in 1965, Smith and Turner demonstrated it [49]. However, the following decades saw little progress and few publications. In the mid-1970's the Q-switch was developed, allowing short pulses with high peak power densities, thereby increasing the applicability of the technique to more materials [49]. But it was in 1987, high $\mathrm{T}_{c}$ superconducting films were successfully grown and this greatly reinvigorated interest in the PLD process and proved it to be a powerful technique [49].

Pulsed laser deposition is a straightforward technique. The setup involves a target holder and substrate holder in a vacuum chamber. An external high intensity laser is focused by lenses through a window in the chamber to the surface of the target, causing it to vaporize. The resulting plume of vaporized target material expands toward the substrate, depositing the film evenly (given proper distance and alignment) $[49,50]$. This is shown schematically in Figure 3.1 and the actual system is shown in Figure 3.2. Since the laser source is external to the chamber, there is a great flexibility in environments created within the chamber whether reactive gases, plasmas or hybrid techniques are used [49].

For our PLD technique, the chamber was under vacuum, and no gases were introduced. The laser source was a Nd:YAG laser emitting a beam with $\lambda=266 \mathrm{~nm}$ from $4^{\text {th }}$ harmonic generation. Deposition rate of the catalyst metal is varied by tuning the laser power. The thickness of the film was monitored in situ precisely using an oscillating quartz crystal film thickness monitor. For the research covered in this dissertation, the metal catalyst targets were either $\mathrm{Fe}$ or $\mathrm{Ni}$, and the substrates were either p-type silicon with $120 \mathrm{~nm} \mathrm{SiO}_{2}$, n-type 1-10 $\Omega$-cm resistivity low resistivity silicon or glass.

\subsubsection{Pretreatment of the Catalyst Films}

Once the films are deposited on the substrates they are loaded into the thermal CVD system, shown schematically in Figure 3.3 and shown in Figure 3.4. It is composed of a quartz tube, a furnace, a carbon source gas, buffer gases, a flow controller and a vacuum pump with temperatures for the furnace set using a temperature controller. Initial experiments controlled gas flow rates using a calibrated needle valve, but this has been replaced with a mass flow controller for more precise control.

Pretreatment takes place as follows. Once loaded, the substrate is annealed at high temperature in a specific gas environment and time period. This process, called pretreatment, causes the metal 


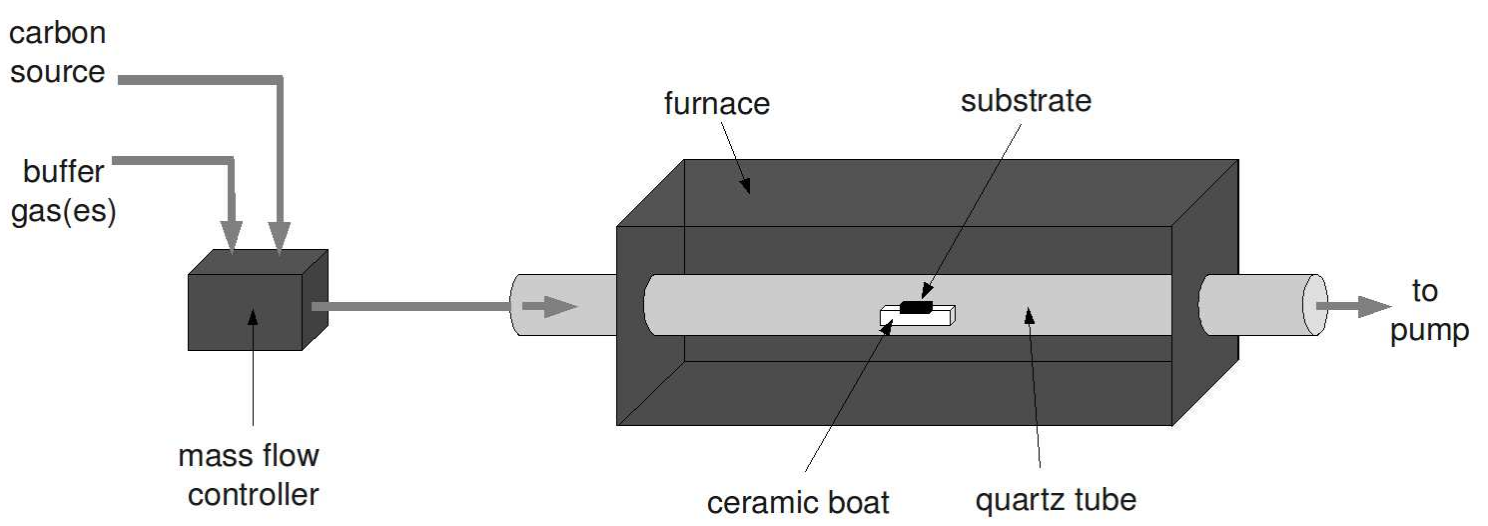

Figure 3.3: Schematic of the thermal CVD setup and components.

atoms in the catalyst film to undergo heat-induced surface diffusion, colliding with one another to form nano-scale catalyst particles. So pretreatment is the process in which the continuous metal film is converted into the nano catalyst particles which will serve as the growth sites for the carbon nanotubes. The pretreatment conditions, along with the catalyst metal and thickness, as we will see, have a large impact on the resulting carbon nanotubes grown.

Typical conditions for our pretreatment are a temperature range of $600-900{ }^{\circ} \mathrm{C}$ in the flow of $\mathrm{H}_{2}+\mathrm{N}_{2}, \mathrm{NH}_{3}$ or $\mathrm{H}_{2}$ gases for periods of $10-60$ minutes. It is important to note that although these pretreatment temperatures are far below the melting points of the metals given in Table 3.1, the films readily melt because as the size of the film decreases, so does the melting point $[51,52]$.

\subsubsection{Carbon Nanotube Growth and Mechanism}

In order to grow the CNTs, a carbon precursor gas is introduced into the furnace after pretreatment has been completed. This gas undergoes catalytic decomposition, releasing the carbon atoms necessary for growth. Commonly a buffer gas, such as $\mathrm{N}_{2}, \mathrm{H}_{2}$ or $\mathrm{NH}_{3}$ is included to dilute the precursor, increase pressure or enhance growth in some way. Unsurprisingly, choosing a carbon precursor gas is a critical decision. Less stable hydrocarbon gases such as acetylene and ethylene are common for MWCNT growth $[44,53,54]$ and more stable hydrocarbons such as methane $[55,56,57,58]$, carbon monoxide $[59,60]$ and alcohols $[61,62]$ are common for SWCNT growth. MWCNTs, which my research focuses on, have larger diameters than SWCNTs meaning the walls have less curvature 


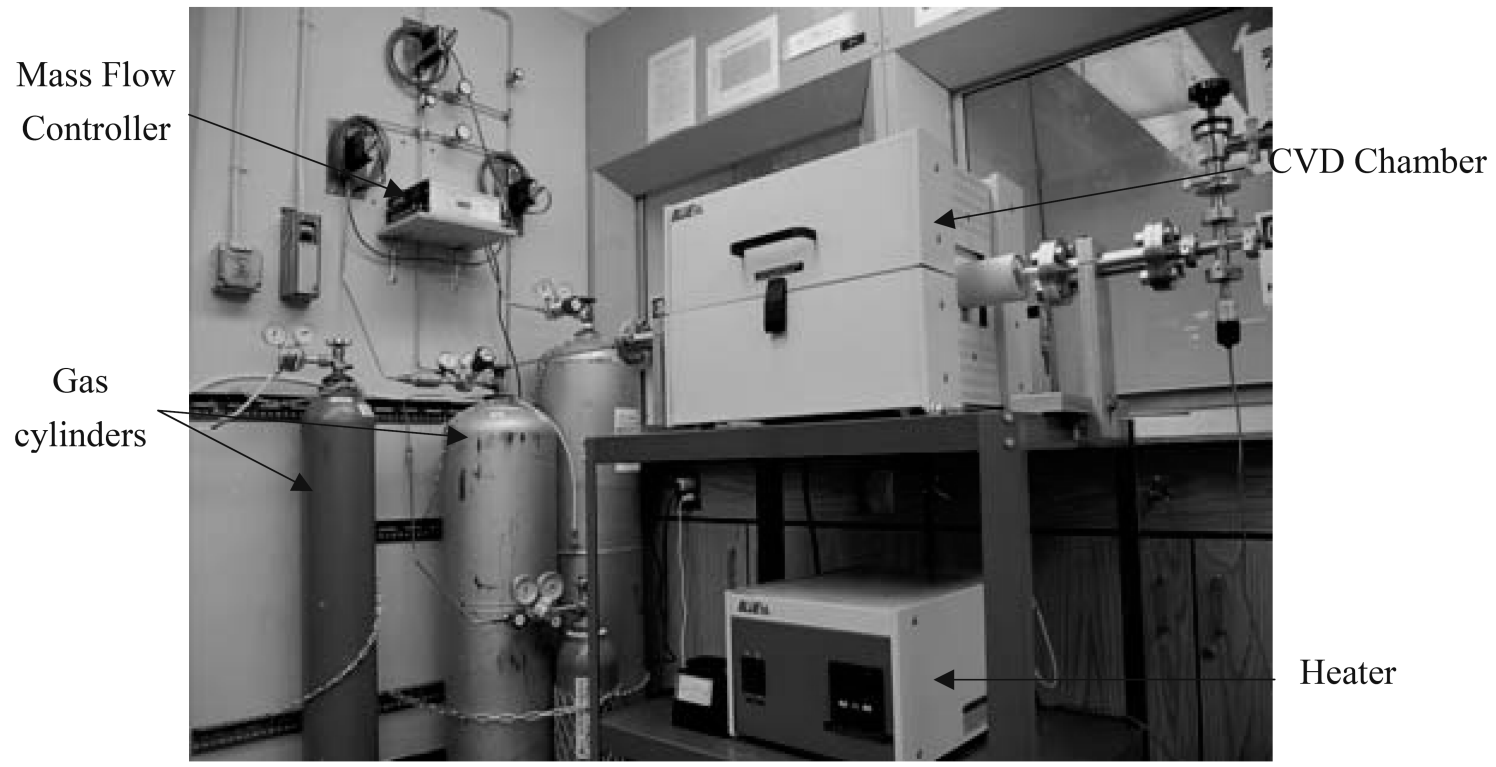

Figure 3.4: The thermal CVD system. Image reprinted with permission from reference [48].

and less strain energy is necessary to form them. The practical side of this is that MWCNTs can be readily grown at lower temperatures than SWCNTs, therefore less stable hydrocarbons are required. For my research, we utilized acetylene as the carbon precursor for thermal CVD growth.

The basic growth mechanism for our TCVD growth is referred to as the Vapour-Liquid-Solid (VLS) mechanism $[63,64]$. This is a broad mechanism that involves decomposition of the hydrocarbon gas (vapour) on the molten surface of the catalyst particle (liquid) which diffuse into the catalyst and, upon super-saturation, segregate into the crystal tubes (solid). The VLS mechanism describes the growth in general terms but does not address necessary specifics such as the decomposition of hydrocarbon molecules on the catalyst particles and on how this is important for continual CNT growth. Vijaya Kayastha et al. proposed a growth model combining VLS with the dissociative adsorption of acetylene; the details of this model are shown schematically in Figure 3.5 [43].

The first step in the growth of the CNTs is adsorption of the acetylene to the Fe catalyst particles, Figure 3.5 (a). Once in contact, the molecule breaks either one of its $\mathrm{C}-\mathrm{H}$ bonds or one of its C$\mathrm{C}$ bonds. Strong hybridization between $\mathrm{C}$ and Fe atoms results in the breaking of the remaining bonds because Figure 3.5 (b) step (D) is, according to first principle calculations, the most stable 
configuration [65]. Once the acetylene is completely decomposed, the carbon diffuses into the Fe particle.

As listed on Table 3.1, the melting point of bulk Fe is much higher than our growth temperatures. However, the melting temperature decreases with increasing amounts of dissolved carbon, to a minimum of $1148{ }^{\circ} \mathrm{C}$ when $16 \%$ (atomic \%) C [66]. Additionally, the melting point drops drastically when the size of the Fe particle shrinks [51, 52]. Give our nanoparticles are on the scale of $10-20$ $\mathrm{nm}$, the melting point will reduce to about $940-1220^{\circ} \mathrm{C}$, as described in Refernce [52]. Lastly, we must consider that the dissociative process the acetylene undergoes is exothermic, releasing $0.82 \mathrm{eV}$ of heat per $\mathrm{C}_{2} \mathrm{H}_{2}$ [65]. In tandem, we believe these three factors lead to the Fe catalyst particles behing molten but with a solid core in the range of our growth temperatures $\left(\sim 600-1000{ }^{\circ} \mathrm{C}\right)$. The absorbtion and diffusion will take place mostly in the liquid region, and will eventually supersaturate, Figure 3.5 (e), which results in the segregation and formation of the crystalline tubes, Figure 3.5 (f). Since the solid core undergoes far less carbon diffusion than the liquid, it does not nucleate CNT shells and leaves the hollow channel in the tube.

The key to all of this is balancing the rates of carbon absorption and segregation in order to achieve good continuous growth. If the rate of carbon influx is too low, super-saturation will be reached slowly and the rate of growth will be hindered. If, however, too much carbon is supplied, amorphous carbon will aggregate and will eventually cover the catalyst particle, preventing further growth.

Lastly, in very general terms, the CNT growth mechanism also take place in one of two modes, either base growth or tip growth [56]. During base growth, the catalyst particle remains adhered to the substrate and the nanotube forms above the particle. For tip growth, the carbon nanotube forms underneath the catalyst particle and, therefore, remains on the tip. Both growth modes are shown schematically in Figure 3.6. Which mode takes place depends on the metal-substrate interaction. For example, if we deposited the Fe catalyst onto pure silicon it would be able to form some iron silicides that would make their interaction strong, and the particle would remain bound to the surface leading to a base growth mode. Our TCVD growth is a base-growth mode. 

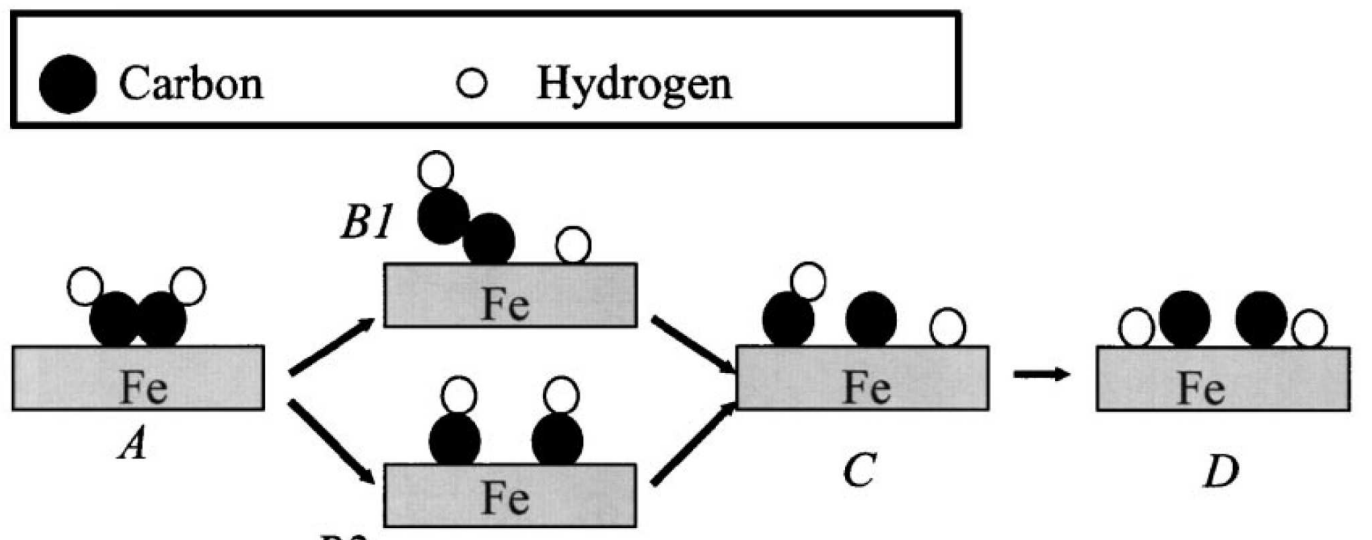

(a)

$$
\text { B2 }
$$

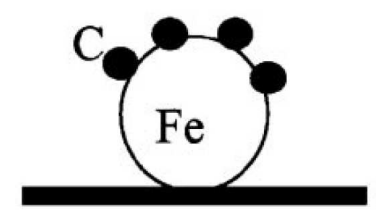

(b)

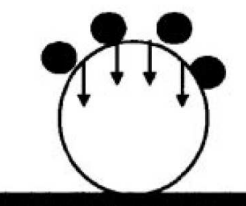

(c)

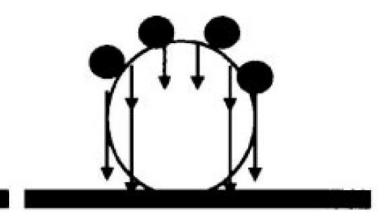

(d)

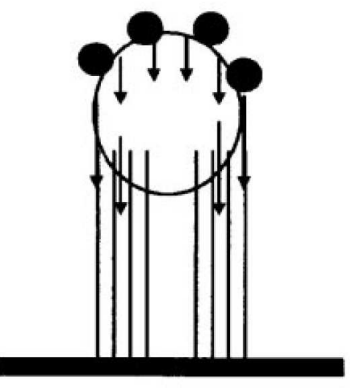

(e)

Figure 3.5: A schematic of the associative adsorption VLS growth mechanism in TCVD growth. (a) Adsorption of the acetylene onto the surface of the Fe catalyst particle. (b) Decomposition of acetylene. (c) Absorption of the carbon into the molten Fe. (d) The carbon atoms diffuse through the liquid Fe. (e) Super-saturation of the Fe with carbon. (f) Segregation of C atoms into the crystalline tubular structure. Reprinted with permission from [43]. Copyright 2004, American Institute of Physics Figure. 

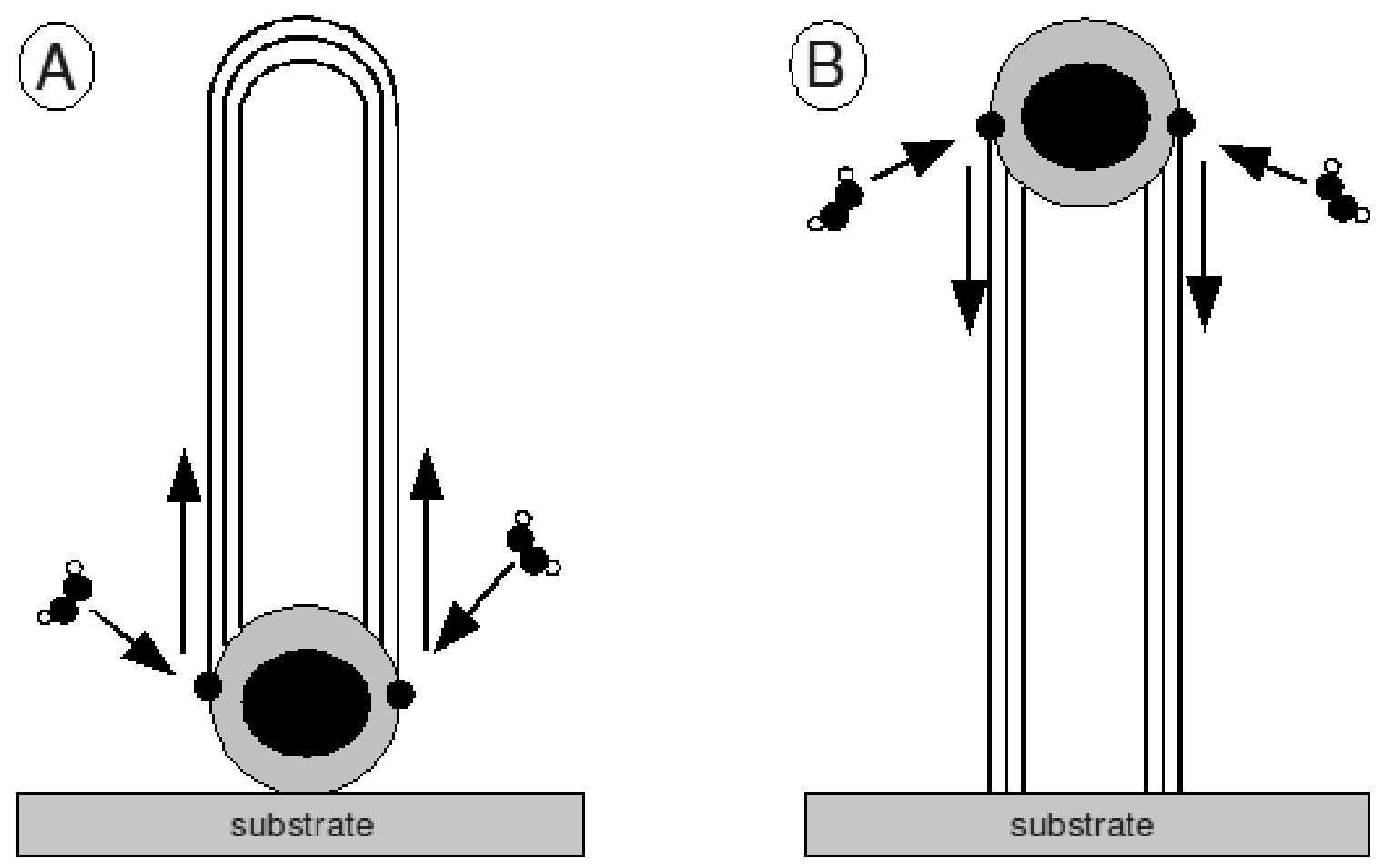

Figure 3.6: (A) In a base growth model the catalyst particle adheres to the substrate and the CNT forms above it. (B) In a tip growth model the carbon forms the hexagonal lattice of the walls underneath the catalyst particle, lifting it from the substrate. 
Table 3.2: Stock TCVD MWCNTs growth conditions

\begin{tabular}{|l|l|}
\hline Substrate & p-type silicon with $120 \mathrm{~nm} \mathrm{SiO}_{2}$ \\
Catalyst Film & $15 \mathrm{~nm} \mathrm{Ni}$ \\
Pretreatment & $300 \mathrm{sccm} \mathrm{H}_{2}, 10$ minutes, $700{ }^{\circ} \mathrm{C}$ \\
Growth & $50 \mathrm{sccm} \mathrm{C}_{2} \mathrm{H}_{2}+120 \mathrm{sccm} \mathrm{H}_{2}, 10$ minutes, $700{ }^{\circ} \mathrm{C}$ \\
Cooling & $300 \mathrm{sccm} \mathrm{Ar}, 45$ minutes \\
\hline
\end{tabular}
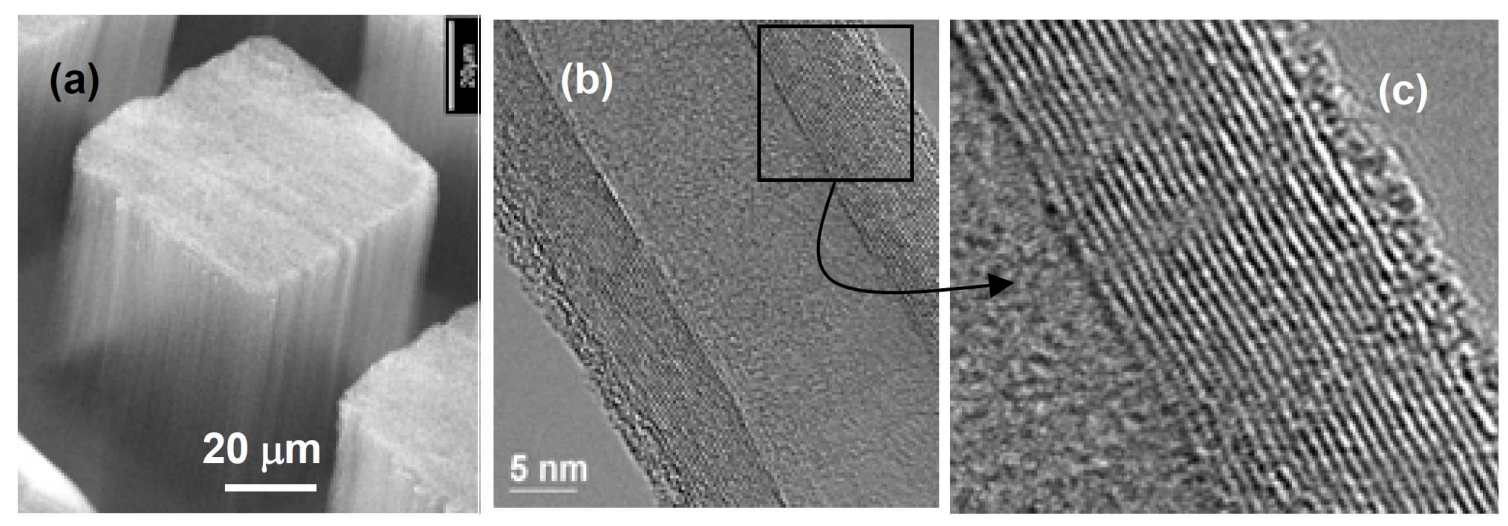

Figure 3.7: Stock TCVD MWCNTs. (a) SEM showing the vertically-aligned stock TCVD MWCNTs grown in arrays by patterning the catalyst film. (b) TEM of a single MWCNT. (c) Wall of a single MWCNT highlighting the high level of graphitic order. Figure reprinted from Reference [67], Copyright IEEE 2008.

\subsection{Stock Thermal CVD MWCNTs}

By the time I joined the research group we had the capability of growing very good TCVD MWCNTs. First, I had to learn to grow these tubes because they would form the stock with which I would carry out future experiments. The conditions for stock growth have varied slightly over the years with changes in the thermal CVD system, but the current stock growth conditions are given in Table 3.2. Images of stock tubes are shown in Figure 3.7 while Raman spectra is shown in in Figure 3.8. In general, the tubes grown in high density and vertical alignment, with lengths of $14 \mu \mathrm{m}$ or longer and diameters of $20-30 \mathrm{~nm}$. The ratio of the graphitic $\left(\sim 1590 \mathrm{~cm}^{-1}\right)$ and disordered $\left(\sim 1350 \mathrm{~cm}^{-1}\right)$ Raman peaks is roughly 1 . 


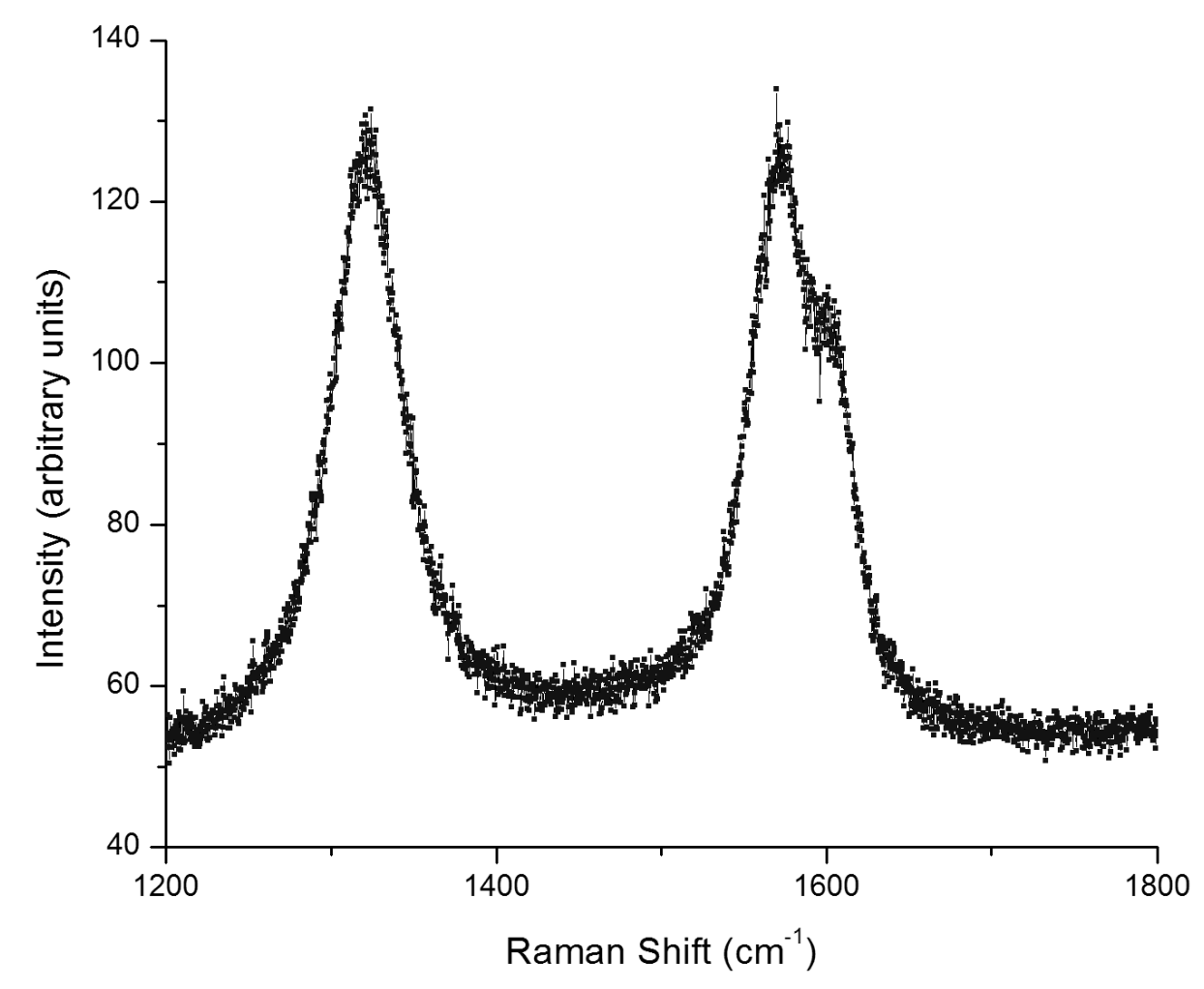

Figure 3.8: Raman spectra of the stock TCVD grown MWCNTs. The ratio of graphitic peak to disorder peak is about 1. 


\subsection{Bamboo-like Multi-walled Carbon Nanotubes}

A problem arises with carbon nanotubes when it is desired to functionalize them: if they retain perfect crystallinity, there are no open bonds to which other molecules can bond. One approach is to grow CNTs and induce defects afterward by methods such as electron beam irradiation [68, 69], ion beam and implantation [70,71], oxidation [72, 73], etc.. Another approach is to grow the CNTs with defects already present. Defects are naturally present in some degree in almost all CNTs, but tend to be random in location. It has been found that CNTs can be grown with a "bamboo" structure, and may contain periodic, open bonds on the sidewalls. Examples of TEM of bamboo CNTs can be found in the work of Lee et al., reference [74].

My first research project in the research group was to examine, along with Vijaya Kayastha, under what conditions we could produce bamboo-like MWCNTs by our thermal CVD system. Research has shown that a variety of catalysts can be used, including $\mathrm{Co}$, Fe and $\mathrm{Ni}[74,75]$ and with a variety

of precursor and carrier gases such as acetylene, methane, ammonia and argon [74, 75, 76]. Because such a large range of possibile conditions and the group's previous experience with MWCNT growth using Fe as the catalyst material, Fe was chosen for our catalyst. Thicker Fe films were used, and unless otherwise noted the results for films of 50 and $100 \mathrm{~nm}$ were identical. The results are discussed below.

The effects of temperature and pretreatment were explored first and the results are compiled in Table 3.3 and shown in Figure $3.9(\mathrm{~A})$ - (D). Pretreatment was always 20 minutes at the growth temperature and a total of $300 \mathrm{sccm}$ pretreatment gas was flowed, however we explored both $\mathrm{NH}_{3}$ and a 3:1 ratio of $\mathrm{H}_{2}+\mathrm{N}_{2}$ as the gas. Growth was conducted for 15 minutes under a combination of $55 \mathrm{sccm}$ of acetylene as the carbon precursor and $123 \mathrm{sccm} \mathrm{NH}$ as the buffer gas. Substrates were p-type silicon with a $120 \mathrm{~nm} \mathrm{SiO}_{2}$ layer. At low temperatures of $750{ }^{\circ} \mathrm{C}$ and below, no bamboo-like CNTs were observed. However, at 900 and $1000{ }^{\circ} \mathrm{C}$ bamboo-like tubes grow readily irrespective of which pretreatment is employed. When the temperature was increased to $1100{ }^{\circ} \mathrm{C}$ no growth takes place because the catalyst particles begin to interact with the $\mathrm{SiO}_{2}$ surface, Figure 3.9 (E). TEM confirmed the bamboo-like nature of the tubes by revealing compartments in the tube structure, Figure $3.9(\mathrm{~F})$. In order to better understand the temperature effect, 800 and $850{ }^{\circ} \mathrm{C}$ growth was investigated. Surprisingly, although lower temperatures show CNT growth and higher temperatures 
Table 3.3: Summary of temperature and pretreatment gas results for bamboo MWCNTs. Pretreatment time was 20 minutes at a flow of $300 \mathrm{sccm}$; growth time 15 minutes with $55 \mathrm{sccm}$ acetylene as carbon source and $123 \mathrm{sccm} \mathrm{NH}_{3}$ as buffer.

\begin{tabular}{|l|l|l|}
\hline Growth/Pretreatment Temp. & $\mathrm{NH}_{3}$ Pretreat & $3: 1 \mathrm{H}_{2}: \mathrm{N}_{2}$ Pretreat \\
\hline $750{ }^{\circ} \mathrm{C}$ & Growth, no bamboo & Growth, no bamboo \\
$800{ }^{\circ} \mathrm{C}$ & Little growth, no bamboo & Little growth, no bamboo \\
$850{ }^{\circ} \mathrm{C}$ & Almost no growth, no bamboo & Almost no growth, no bamboo \\
$900{ }^{\circ} \mathrm{C}$ & Growth, bamboo & Growth, bamboo \\
$1000{ }^{\circ} \mathrm{C}$ & Growth, bamboo & Growth, bamboo \\
$1100{ }^{\circ} \mathrm{C}$ & No growth & No growth \\
\hline
\end{tabular}

show bountiful bamboo-like CNT growth, $800{ }^{\circ} \mathrm{C}$ showed almost no growth and $850{ }^{\circ} \mathrm{C}$ showed no growth at all, Figure 3.10. Clearly a transition is taking place in the catalyst particle formation between 750 and $900{ }^{\circ} \mathrm{C}$. For bamboo-like growth, then, the desired temperature window is $900-$ $1000{ }^{\circ} \mathrm{C}$.

The immediate conclusions are that higher temperatures are better for forming the catalyst conditions necessary for bamboo-like growth. However, we next repeated the same experiments, changing the pretreatment gas to Ar. These experiments showed no bamboo growth, indicating clearly that nitrogen's presence is critical for bamboo. An example of MWCNTs grown via Ar pretreatment is shown in Figure 3.11 and clearly lacks bamboo-like CNTs indicating that nitrogen is critical to the process.

The next logical step is to see the effect of different buffer gases for the growth phase. Here the temperature was $900{ }^{\circ} \mathrm{C}$, pretreatment was $300 \mathrm{sccm} \mathrm{NH}$ for 20 minutes, as before, but the buffer gas flowed with acetylene was varied. Argon growth, 3.12 (B), shows a drastic reduction in the number of tubes with bamboo-like character; this was expected because of the importance of nitrogen. However, we see that if we flow $\mathrm{N}_{2}$ as the buffer gas the growth is almost completely ruined, $3.12(\mathrm{C})$. It appears that although the presence of nitrogen is critical to forming the proper catalysts for bamboo-like CNTs, too much will poison the growth. According to the literature, the inclusion of nitrogen in the process appears to enhance the bamboo-nature by incorporation into the carbon lattice; nitrogen introduces pentagonal defects causing curving in the sidewalls $[77,78]$. This also explains why too much nitrogen will poison growth, as too much nitrogen keeps the carbon lattice from forming. 

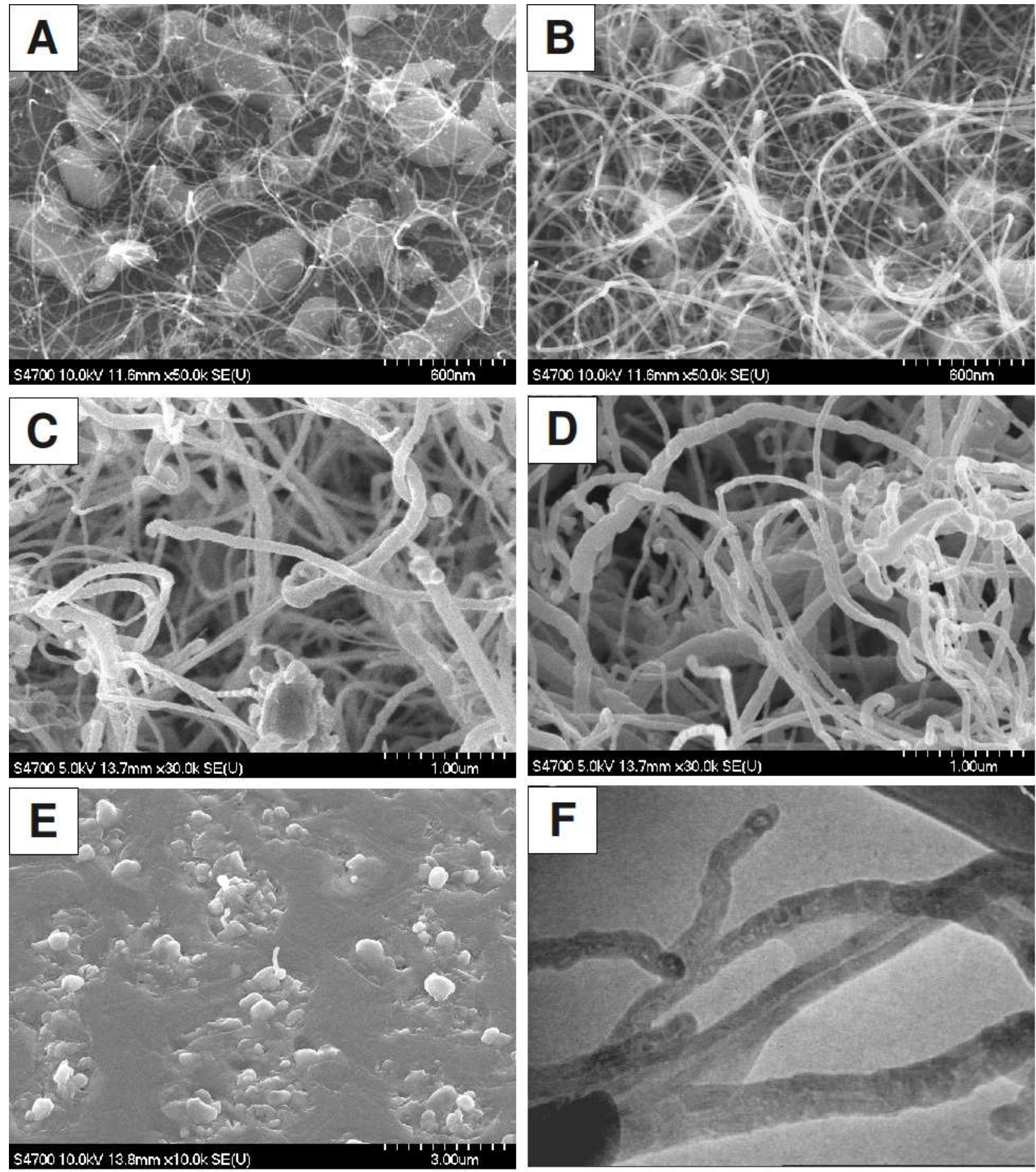

Figure 3.9: At or below $750{ }^{\circ} \mathrm{C}$ with $\mathrm{NH}_{3}$ as a buffer during growth, MWCNT growth was sparse and no bamboo-like tubes were observed either in (A) $\mathrm{NH}_{3}$ or (B) $\mathrm{H}_{2}+\mathrm{N}_{2}$ pretreatment conditions. At 900 (not shown) and $1000{ }^{\circ} \mathrm{C}$, bamboo-like tubes are numerous and readily grown in both $(\mathrm{C})$ $\mathrm{NH}_{3}$ and (D) $\mathrm{H}_{2}+\mathrm{N}_{2}$ pretreatment. (E) At $1100{ }^{\circ} \mathrm{C}$ the $\mathrm{SiO}_{2}$ surface begins reacting with the catalyst film, preventing growth. (F) TEM image revealing compartments in the tube structure. 

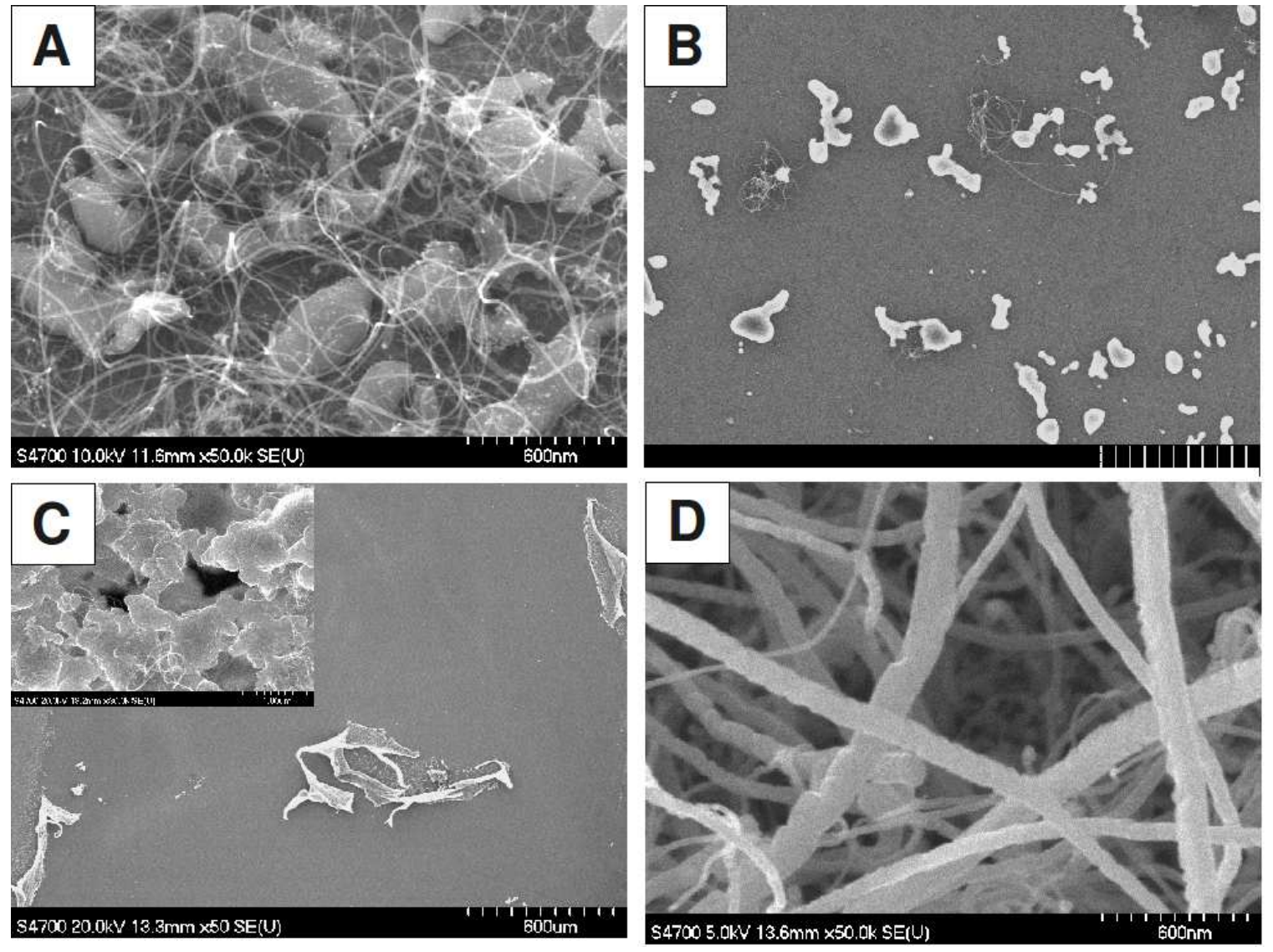

Figure 3.10: (A) Non-bamboo CNTs grow readily at $750{ }^{\circ} \mathrm{C}$. (B) At $800{ }^{\circ} \mathrm{C}$ growth is reduced as the catalyst forms into much larger clumped clusters. (C) At $850{ }^{\circ} \mathrm{C}$ growth is completely suppressed as massive catalyst particles separated by large differences dominate. (D) At $900{ }^{\circ} \mathrm{C}$ we observe growth of many carbon nanotubes of bamboo-like character. 

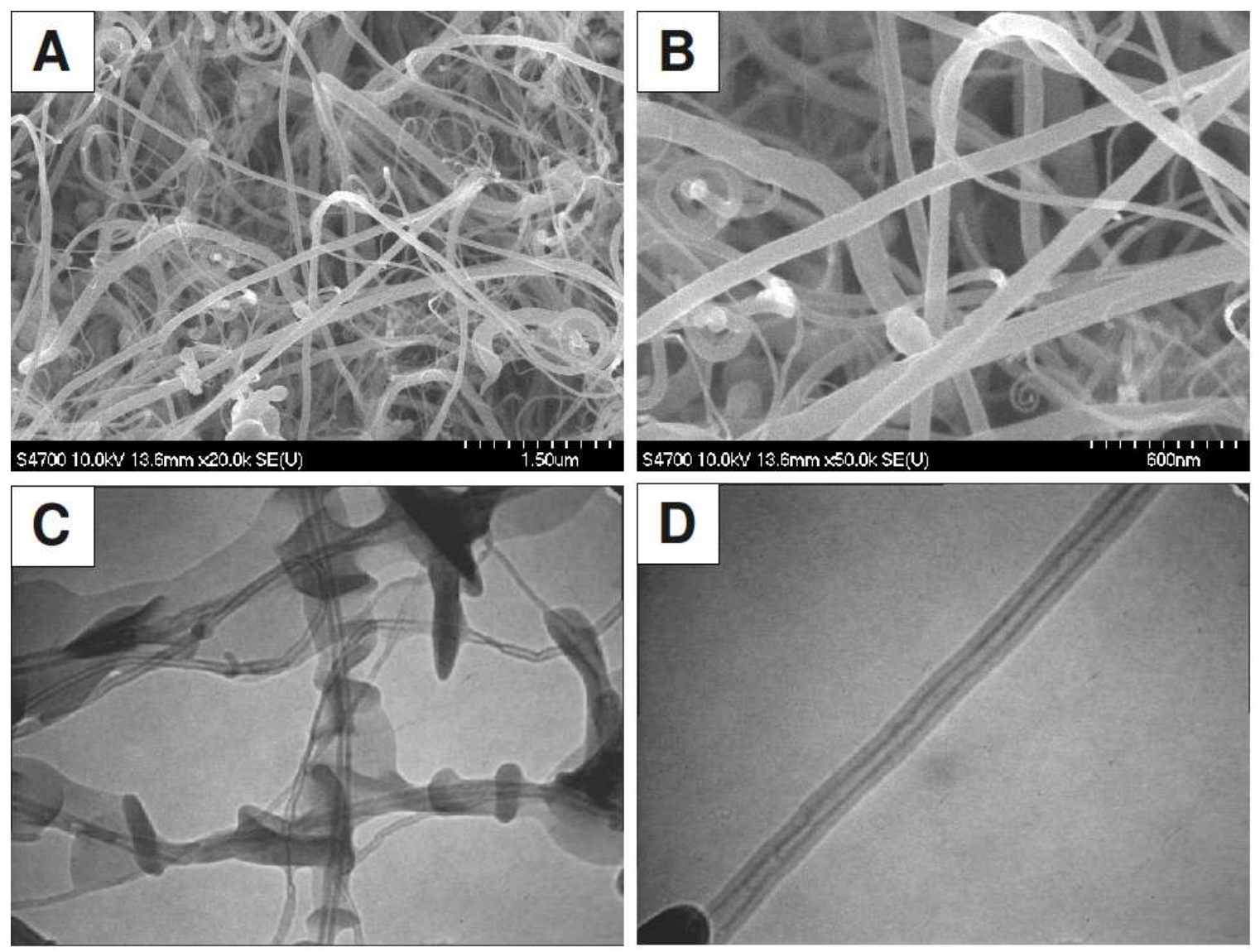

Figure 3.11: Catalyst films pretreated with $\mathrm{Ar}$ instead of $\mathrm{NH}_{3}$ or $\mathrm{H}_{2}+\mathrm{N}_{2}$ give way to MWCNTs with a lack of bamboo-like character. (A) - (B) SEM images of the growth at $900{ }^{\circ} \mathrm{C}$ with $\mathrm{NH}_{3}$ buffer gas. (C) - (D) TEM showing the interior channels.

Our ideal conditions are summarized in Table 3.4.

For certain applications, such as using the MWCNTs as field emitters or nanoelectrodes, it is desirable to have the MWCNTs grown directly on substrates that are conductive. To this end, we repeated our experiments to ensure we had the capability of making the bamboo MWCNTs grow directly on low resistance silicon substrates. Once again, pretreatment was always 20 minutes at the growth temperature of $900{ }^{\circ} \mathrm{C}$ with a total of $300 \mathrm{sccm}$ pretreatment gas, 15 min growth under a combination of $55 \mathrm{sccm}$ of acetylene as the carbon precursor and $123 \mathrm{sccm} \mathrm{NH}_{3}$ as the buffer gas. Surprisingly, we found that when the $\mathrm{H}_{2}$ and $\mathrm{N}_{2}$ mix was used, growth was almost completely suppressed. However, when pretreating with $\mathrm{NH}_{3}$, growth matched the $\mathrm{Si} / \mathrm{SiO}_{2}$ substrates; both 

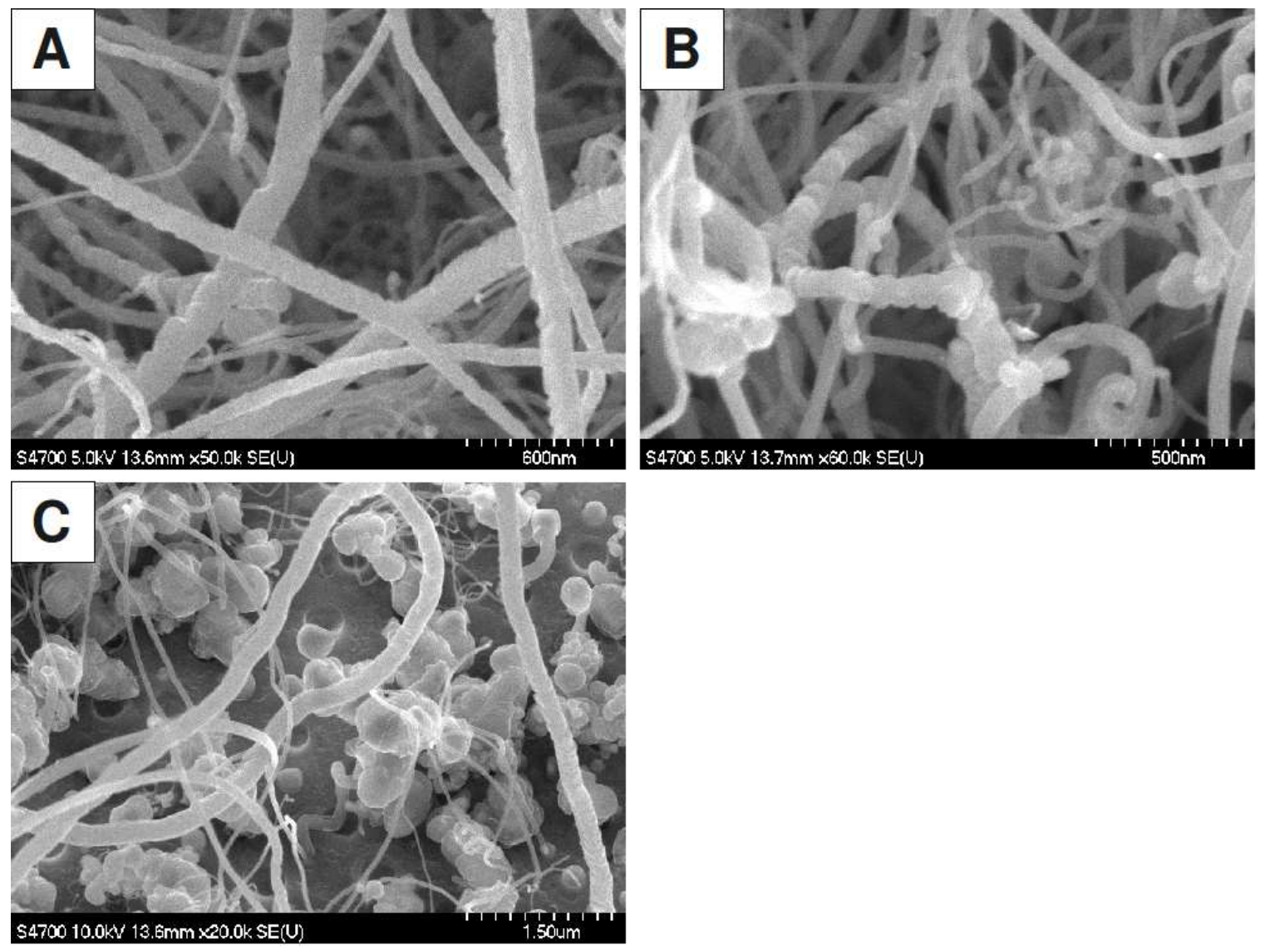

Figure 3.12: The effect of different buffer gases on growth. (A) $\mathrm{NH}_{3}$ - almost all tubes are bamboolike. (B) $\mathrm{Ar}$ - a much lower percentage of tubes show bamboo-like character. (C) $\mathrm{N}_{2}$ - growth of any kind is suppressed leaving sporadic growth.

Table 3.4: Summary of the ideal bamboo conditions for our thermal CVD chamber.

\begin{tabular}{|l|l|}
\hline Substrate & p-type Si with $120 \mathrm{~nm} \mathrm{SiO}$ \\
Catalyst & PLD deposited $50-100 \mathrm{~nm} \mathrm{Fe}$ \\
Temperature & $900-1000{ }^{\circ} \mathrm{C}$ \\
Pretreatment & $20 \mathrm{~min}, 300 \mathrm{sccm} \mathrm{NH} \mathrm{NH}_{3}$ or $3: 1 \mathrm{H}_{2}: \mathrm{N}_{2}$ \\
Growth & $15 \mathrm{~min}, 55 \mathrm{sccm}$ acetylene and $123 \mathrm{NH}_{3}$ \\
\hline
\end{tabular}




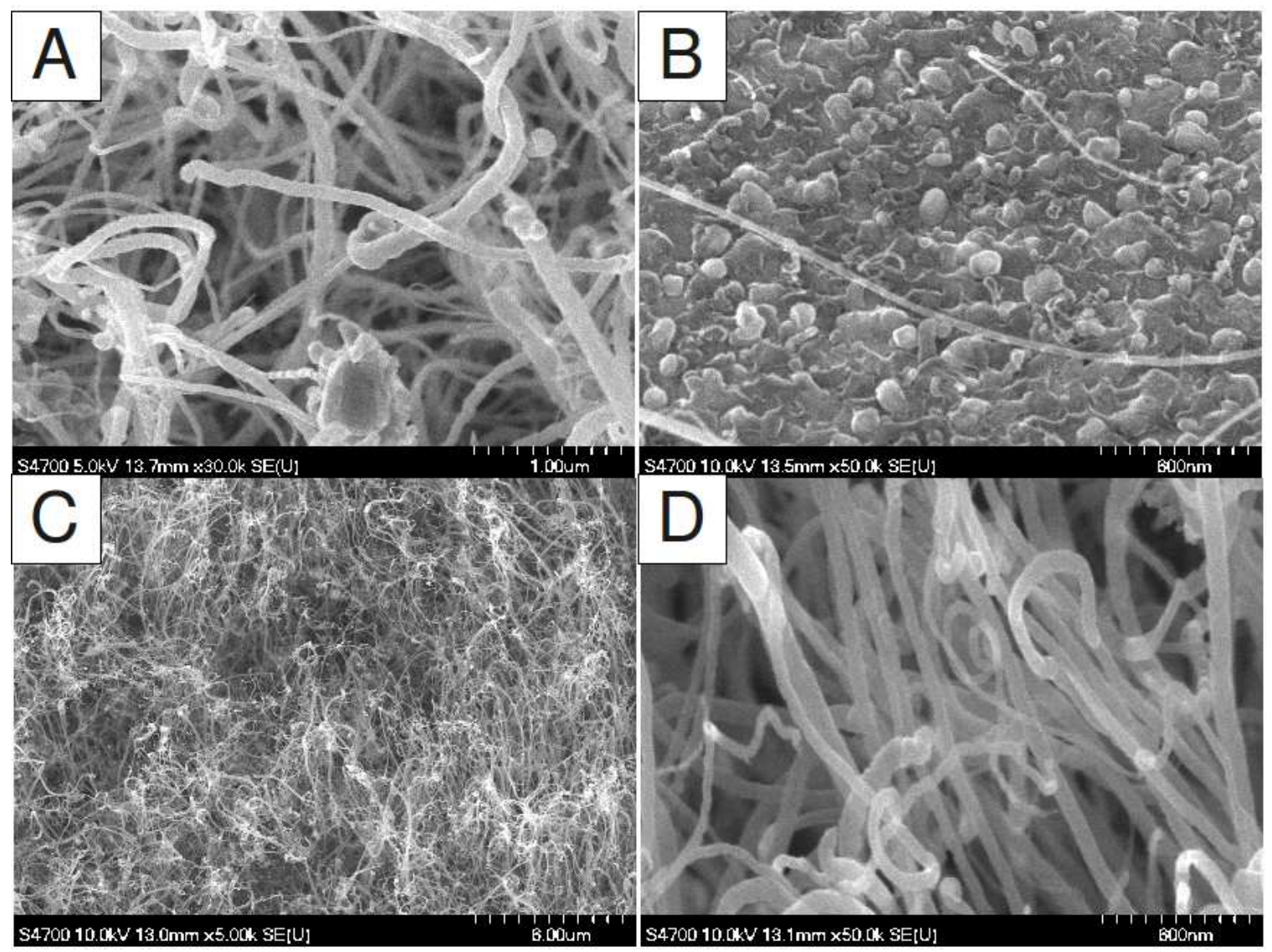

Figure 3.13: (a) Our best bamboo conditions on p-type $\mathrm{Si}$ with $120 \mathrm{~nm} \mathrm{SiO}_{2}$ are the same with $\mathrm{NH}_{3}$ or $3: 1 \mathrm{H}_{2}: \mathrm{N}_{2}$ pretreatment. Growth on low resistance with (b) $3: 1 \mathrm{H}_{2}: \mathrm{N}_{2}$ pretreatment shows almost no growth while (c)-(d) full growth take place after $\mathrm{NH}_{3}$ pretreatment.

results are shown in Figure 3.13. So while we are able to reproduce bamboo growth similar to the $\mathrm{Si} / \mathrm{SiO}_{2}$ substrates, the low resistance silicon growth is much more sensitive to the chemistry of the pretreatment gas. Investigating this further is beyond the scope of the present research. It should be noted that in following experiments, we found the bamboo MWCNTs break easily into very small lengths during ultrasonication which, unfortunately, limits their use in other projects.

\subsection{Plasma-Enhanced CVD Growth and Stock MWCNTs}

A second growth method is used to grow MWCNTs for our experiment. Plasma-enhanced chemical vapor deposition (PECVD) is a process similar to TCVD in that they are both VLS mechanisms. 
Our PECVD method uses dual radio frequency plasma generators, as shown in Figure 3.14. The top generator is used to transform our hydrocarbon precursor gas into a plasma, leaving our carbon in an ionic form. The second generator puts a bias on the associated electrode, which is in electrical contact with our substrate; this bias gives the substrate a negative charge, causing the positive carbon ions in the plasma to be attracted directly to the substrate. The details of the growth are decidedly more complicated because of two factors: (1) the plasma dissociates the gas before adsorption (2) the bias causes ions to impact the substrates and the growing MWCNTs with quite a bit of energy and, so the growth mechanism is not fully understood [79]. As with TCVD care must be taken to find the correct values for all parameters to ensure good growth. Bias is one of these new critical parameters which must be tuned carefully, because higher bias aligns the CNTs during growth, but too much results in resputtering and hampers the growth [80]. Predominantly acetylene, ethylene and methane are used as the carbon precursors for PECVD methods [79, 80].

As with our TCVD growth, we begin with catalyst-coated silicon substrates - note that growth works equally well on $\mathrm{SiO}_{2}$, silicon or low-resistance silicon. We use a dual PECVD chamber setup, Figure 3.14, that consists of two electrodes for a dual radio-frequency (RF) plasma. In this case, the top plasma is used to drive the decomposition of the source gas (methane) and the bottom plasma is used to initiate a bias voltage that accelerates the growth species toward the substrate, giving us separate control over primary gas decomposition and substrate biasing. The top electrode operates in RF mode while the bottom electrode operates in DC mode.

A metal plate holds the substrates in place on a boron-nitride ceramic heater; the plate is electrically connected to the bottom plasma by a wired connection to the bottom electrode and shares the same bias. The heater and its electrical connections are ceramic-coated to electrically isolate then from the plasma. A thermocouple is embedded inside the ceramic and in contact with the heater on which the substrates rest. A temperature controller is connected to the thermocouple to calibrate the heater temperature. Precise adjustment of gas flow into the PECVD chamber is accomplished by a mass flow controller. The system is designed to control the flow of different process gases simultaneously.

The stock PECVD tubes are $2-3 \mu \mathrm{m}$ in length with widths of roughly $50 \mathrm{~nm}$. Due to the electric field they grow in extreme vertical alignment. The hostile plasma environment ensure the graphitic order is lower than the TCVD tubes, as shown in Figure 3.16, and given their relatively 


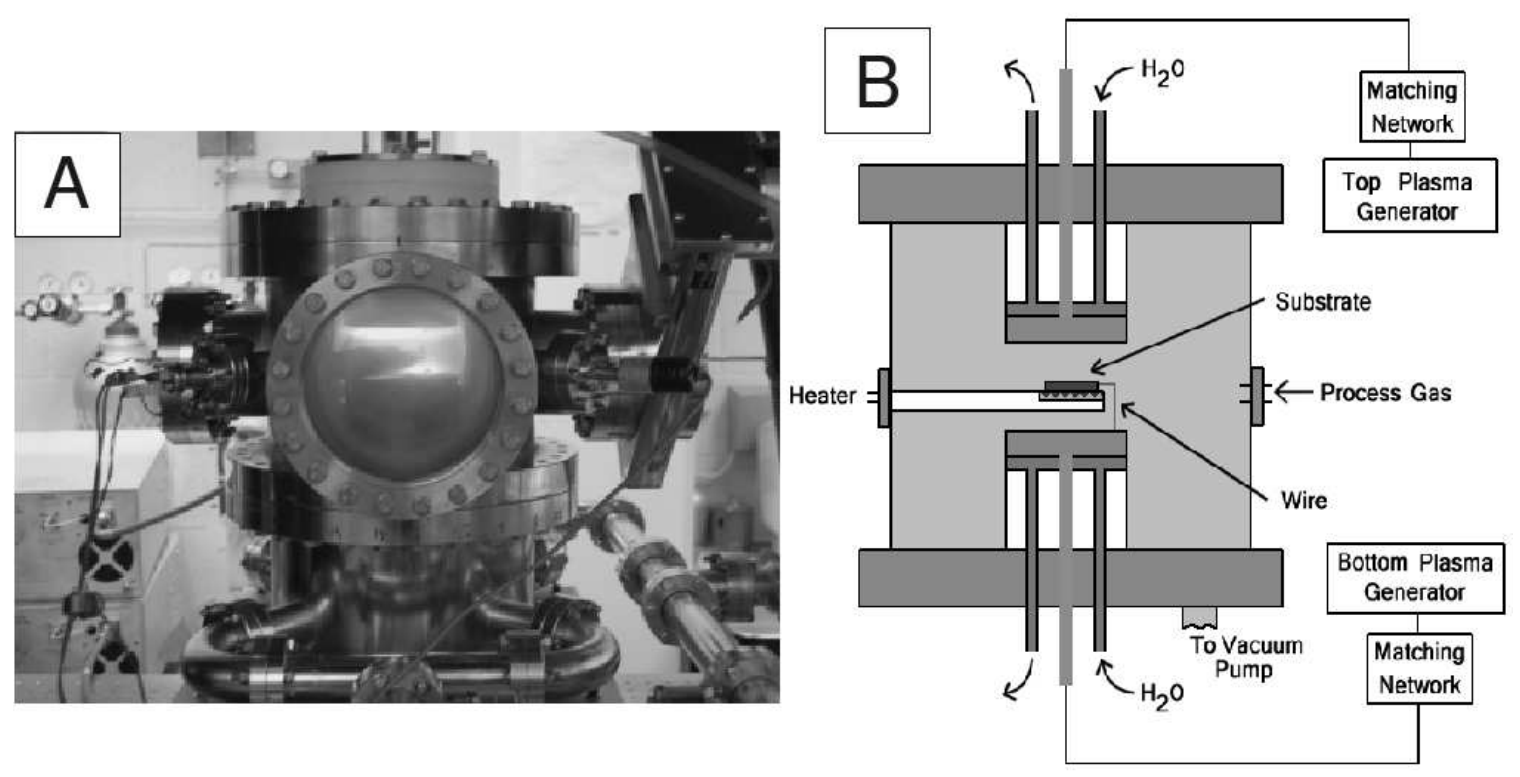

Figure 3.14: (a) The PECVD growth chamber. (b) Schematic of the chamber, showing the inner workings. Reprinted with permission from [81].

Table 3.5: Stock PE-CVD MWCNTs growth conditions

\begin{tabular}{|l|l|}
\hline Substrate & p-type silicon with $120 \mathrm{~nm} \mathrm{SiO}_{2}$ or n-type $1-10 \Omega$-cm resistivity \\
Growth & $125 \mathrm{sccm} \mathrm{CH}_{4}, 60$ minutes, $450{ }^{\circ} \mathrm{C}$ \\
Top Plasma Power & $150 \mathrm{~W}$ \\
Bottom Plasma Bias & $-150 \mathrm{~V}$ \\
\hline
\end{tabular}

small aspect ratio they tend to remain rigidly vertical under normal conditions. These properties make them ideal for any application which requires vertical arrays or for which tangling of long tubes is undesired. In general this makes them easier to deal with for dispersion and experiments tend to always involve the PECVD tubes first. The growth conditions are summarized in Table 3.5 and the tubes are shown in Figure 3.15.

\subsection{Summary}

Growth of bamboo MWCNTs, which have defective, ridged sidewalls, was demonstrated by a thermal CVD process. Nitrogen is necessary to the growth, but too much nitrogen suppresses all growth. In 

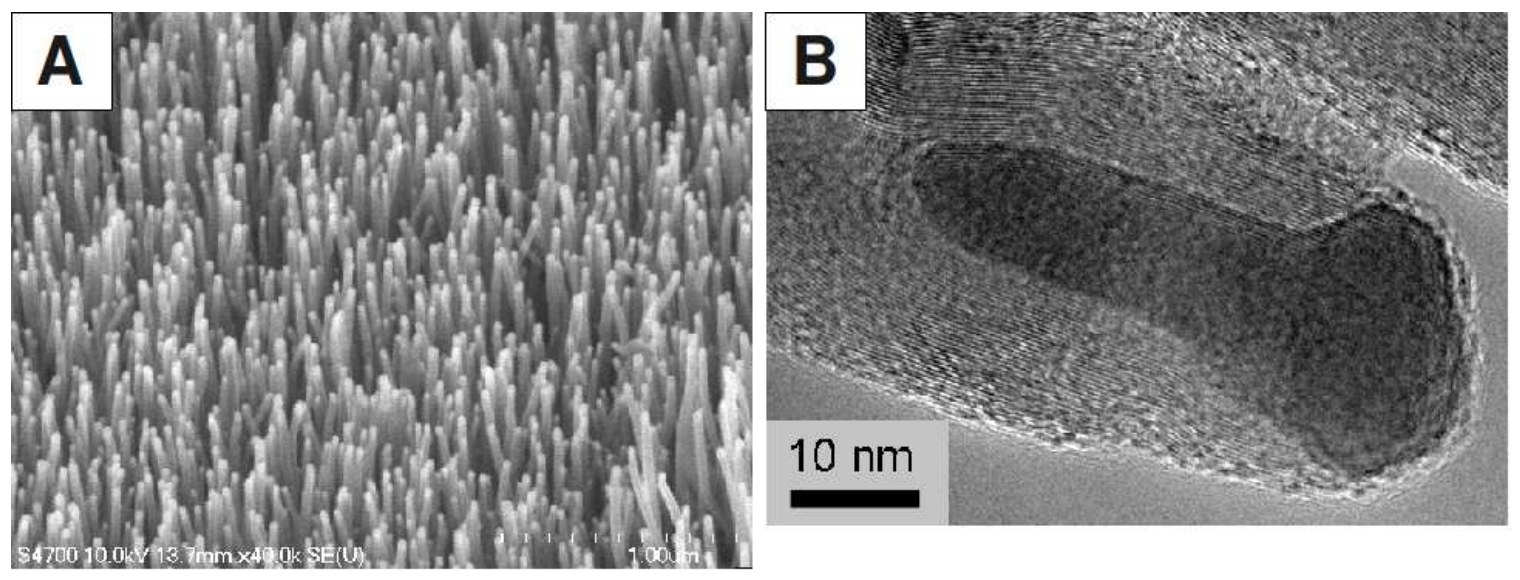

Figure 3.15: Stock PECVD grown MWCNTs. (a) SEM micrograph of the vertically aligned asgrown tubes. (b) TEM highlighting the crystalline nature of the walls and the embedded Ni catalyst particle.

the following experiments, we found the bamboo MWCNTs break too easily into very small pieces during ultrasonication which, unfortunately, limits their use in other projects. The chemistry seems to be somewhat different between $\mathrm{SiO}_{2}$ and low-resistance silicon surfaces, but we have succeeded on both. In addition, the growth conditions and characteristics of the "stock" TCVD and PECVD MWCNTs were described. The stock MWCNTs will serve as the base MWCNTs for the following projects. 


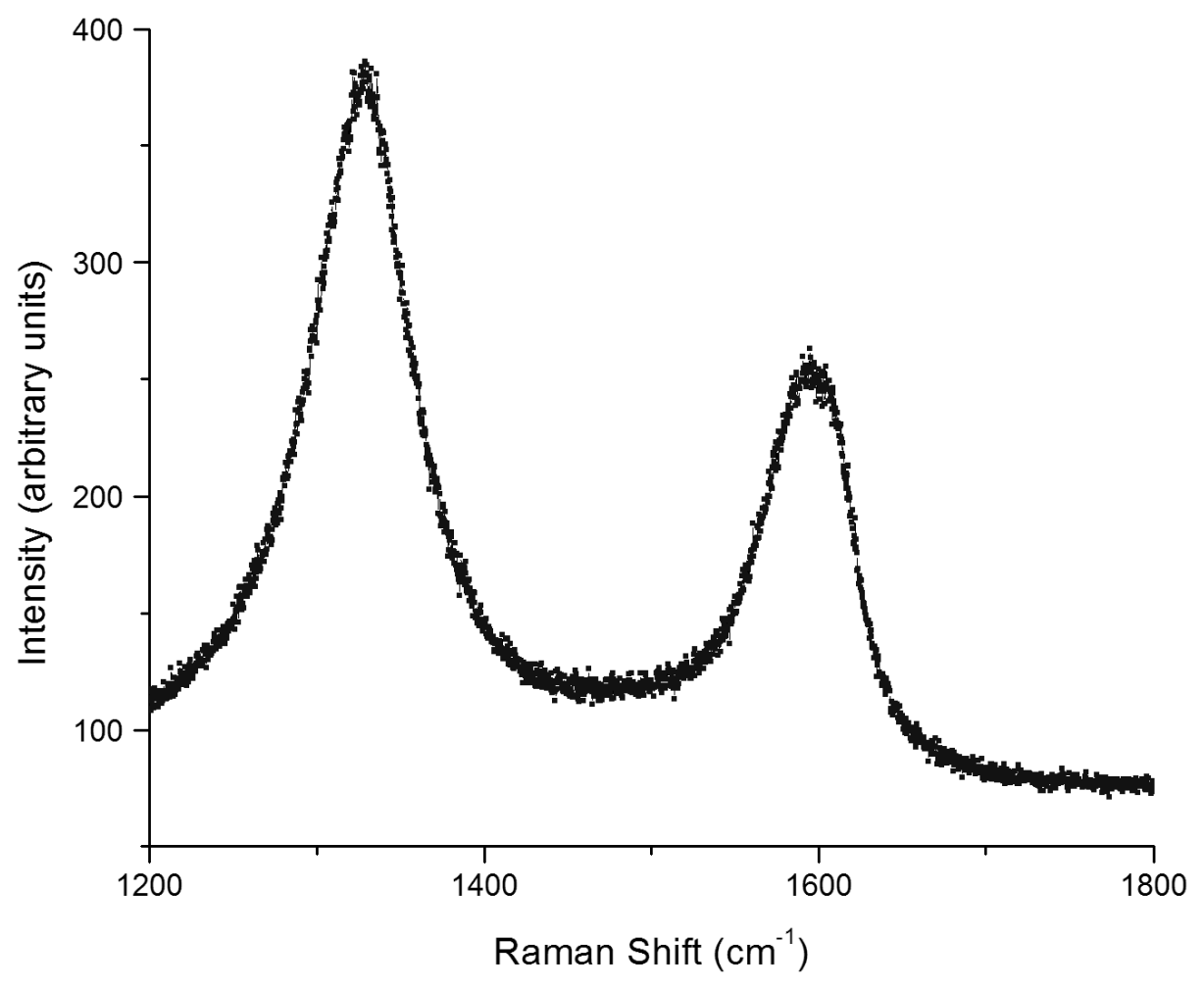

Figure 3.16: Raman spectra of the PECVD grown MWCNTs. These tubes are considerably less graphitic and more disordered than the TCVD tubes. 


\section{Chapter 4}

\section{Horizontal Dispersions \&}

\section{Dielectrophoretic Deposition}

Carbon nanotubes are used in a wide variety of devices, including, but not limited to, field effect transistors $[14,15,16,17,18]$, chemical and biological sensors [19, 20], scanning probe microscopy tips [21, 22, 23] and field emitters [24]. However, for any CNT device, the most basic and important challenge is proper placement. Initial investigations into placing CNTs by gas flow, spin coating, electrophoresis, surfactant-assisted dispersions and dielectrophoresis. Based on these experimental tests, we chose to focus on surfactant-assisted dispersions and dielectrophoresis, which are discussed in this chapter.

\subsection{Horizontal Dispersion}

\subsubsection{Introduction}

The extremely low solubility of CNTs in conventional solvents is continually a road block, hindering the progress of CNT-based applications [82]. Due to strong van der Waals interactions between CNTs, the tubes aggregate into bundles in conventional solvents [83], and research is underway looking for methods to create homogeneous dispersions $[84,85,86]$. In order to use the carbon nanotubes we grow, we must separate them from one another. The thermal-CVD MWCNTs, in 
particular, are long, flexible and tend to become tangled in solution. In this section, a surfactantassisted approach to disperse the tubes is investigated. This work was a research project conducted along with Shun Wu for her Masters Degree thesis [48] and with the collaboration of undergraduate Robert Niffenegger.

\subsubsection{Mechanism and Goals}

Many approaches have been reported to make quality CNT dispersions, including both physical and chemical methods $[84,85,86]$. Physical methods generally involve only mechanical ultrasonication or centrifugation. This often results in shortening of the tubes and, in the case of centrifugation, a massive reduction in tube concentration, which is not favorable to most device applications. The chemical approach entails coating the CNTs with surfactants or polymers that assist the dispersion process $[84,85,86,87]$. While these techniques are successful in enhancing CNT solubility, they also functionalize the MWCNTs, altering their properties [88].

Our goal is to disperse thermal-CVD grown MWCNTs without damaging their structure or changing their properties (including length). The approach is based on reports of non-destructive methods involving ambiphilic surfactants interacting non-covalently $(\pi-\pi$ bonding) with the CNT surface [89]. The ambiphilic nature of these surfactants comes from their structure where one end of the molecule is hydrophobic in nature (and interacts with the CNTs) and the other is hydrophilic (and interacts with the solvent) [90]. Importantly, most of these surfactants have a low melting point of $200-300{ }^{\circ} \mathrm{C}$ and can be removed by oxygen annealing at temperatures low enough to preserve the CNTs [91]. We chose to use Sodium Dodecyl Sulfate (SDS) (Johnson Matthey), a charged surfactant that will help separate the CNTs in solution by repulsion of the charged hydrophobic ends, which also enhance the CNT solubility by creating a hydrophilic "shell" around the tube [87, 92].

Creating the MWCNT dispersion was done as follows. A scalpel was used to remove $1 / 4$ of the as-grown TCVD MWCNTs (stock conditions) from a substrate of dimensions $1.6 \mathrm{~cm} \times 1.1 \mathrm{~cm}$ and were placed directly into a bottle containing $10 \mathrm{~mL}$ of 200 proof ethanol. The large clumps of CNTs were initially separated by shaking the solution for $2-3$ minutes, after which it was divided equally into ten separate containers. A varying number of drops $(\sim 1 / 30 \mathrm{~mL}$ each) of surfactant solution (concentration $0.05 \mathrm{mg} / \mathrm{mL}$ ) were added to each bottle, then ethanol was then added until the total volume was $10 \mathrm{~mL}$. Before dispersing the solution onto the silicon substrates, the bottles 


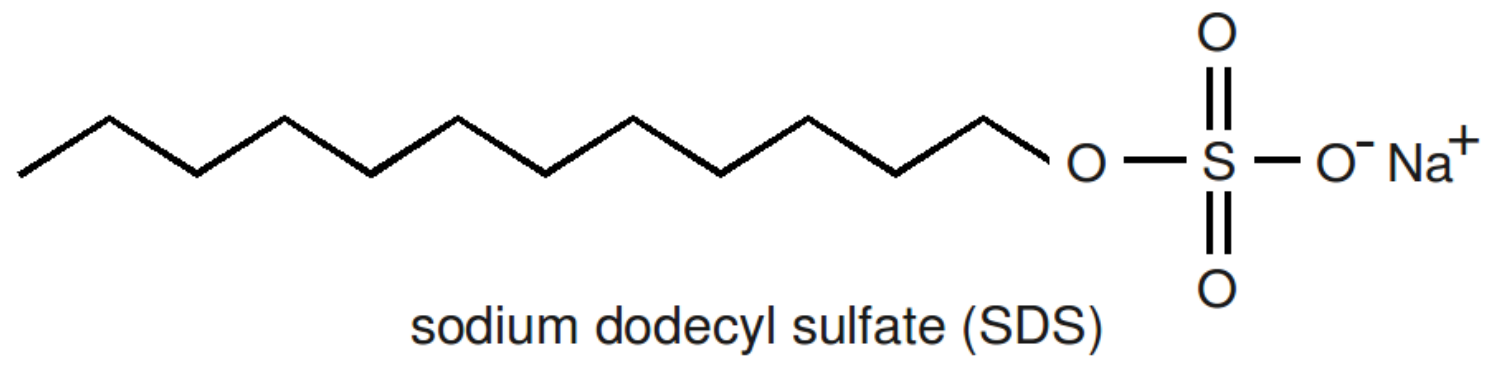

Figure 4.1: Chemical structure of sodium dodecyl sulfate, $\mathrm{CH}_{3}\left(\mathrm{CH}_{2}\right)_{11} \mathrm{OSO}_{3} \mathrm{Na}$.

Table 4.1: SDS Experimental Parameters

\begin{tabular}{ccccc}
\hline \multirow{2}{*}{ Solution Number } & \multicolumn{3}{c}{ Surfactant } & \multirow{2}{*}{ Sonication } \\
\cline { 2 - 4 } & Drops & Weight & Concentration & \\
\hline \hline 1 & 20 & $0.033 \mathrm{mg}$ & $11.4 \mu \mathrm{M}$ & $15,30 \mathrm{~min}$ \\
2 & 40 & $0.0667 \mathrm{mg}$ & $23.1 \mu \mathrm{M}$ & $15 \mathrm{~min}$ \\
3 & 80 & $0.1333 \mathrm{mg}$ & $46.2 \mu \mathrm{M}$ & $15 \mathrm{~min}$ \\
4 & 120 & $0.2 \mathrm{mg}$ & $69.3 \mu \mathrm{M}$ & $15,30,60 \mathrm{~min}$ \\
5 & 160 & $0.2667 \mathrm{mg}$ & $92.4 \mu \mathrm{M}$ & $15,30,60 \mathrm{~min}$ \\
\hline
\end{tabular}

were treated in an ultrasonicating bath for differing lengths of time (130 Watt Ultrasonic Processor, Sonics \& Materials Inc.). The experimental conditions are summarized in Table 4.1.

\subsubsection{Effect of SDS Concentration}

For this series of experiments, the ultrasonication time was limited to 15 minutes. Representative SEM images for each concentration are presented in Figure 4.2. The trend shows that dispersion is enhanced with increasing SDS concentration, up to 120 drops $(69.3 \mu \mathrm{M})$ after which we observe no improvement and merely more SDS deposited on the substrate. Too little surfactant means the MWCNTs are not sufficiently modified by SDS molecules to reach optimal dispersion. However, there is an SDS concentration that is reached at which each MWCNT is fully covered in SDS and can no longer accept more molecules. In order to get a more generally usable result, we now estimate the weight ratio of MWCNTs to surfactant for the optimal condition, as follows.

As mentioned earlier, the growth area of the MWCNTs was $1.6 \mathrm{~cm} \times 1.1 \mathrm{~cm}$ and each bottle receives $1 / 10$ of $1 / 4$ of this growth area, or $0.044 \mathrm{~cm}^{2}$. The height of the VA-MWCNTs were estimated from SEM images taken at a $45^{\circ}$ tilt $(\sim 42 \mu \mathrm{m})$; the density of graphite, $2.0 \mathrm{~g} / \mathrm{cm}^{3}$ was 


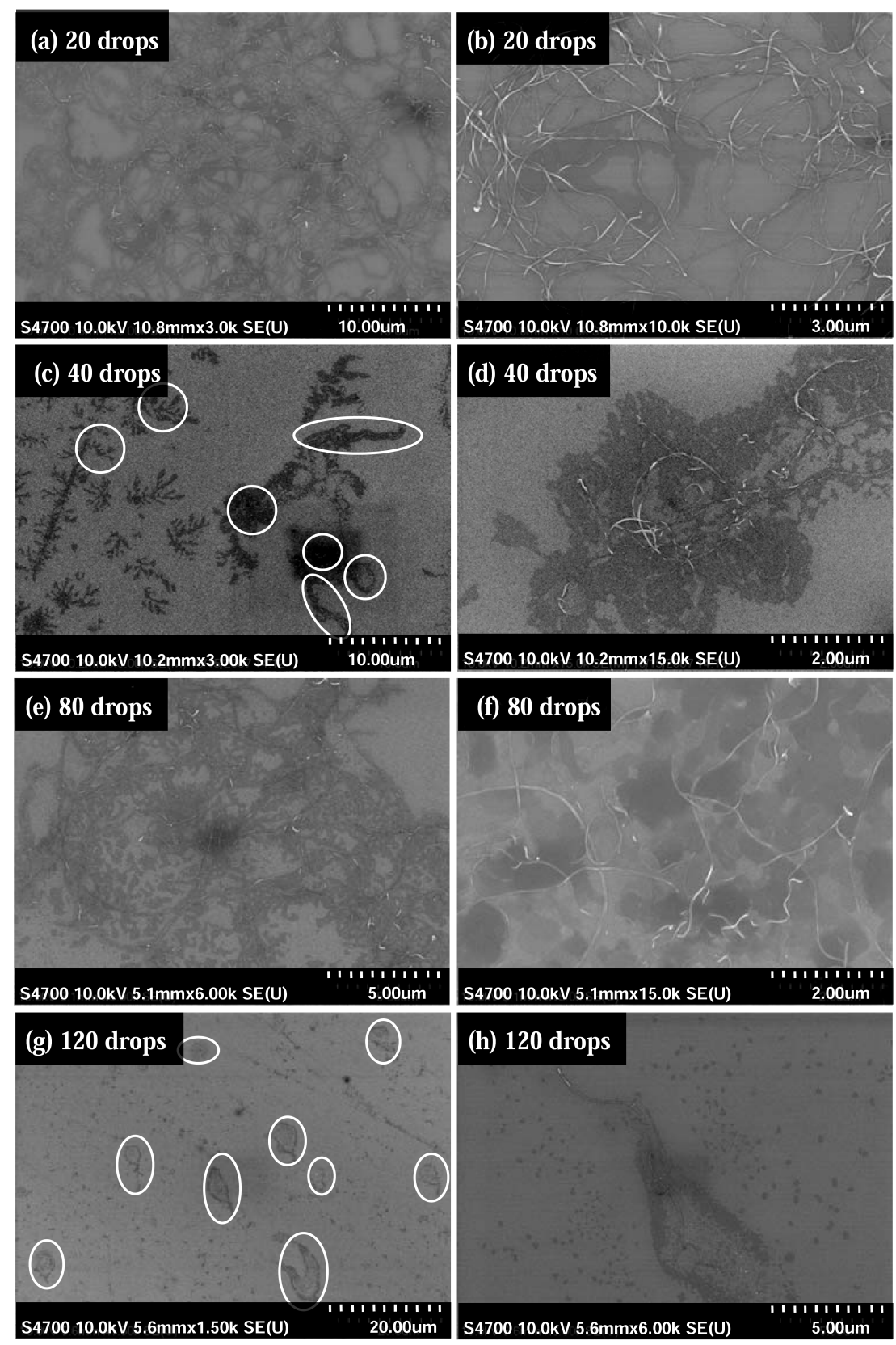

Figure 4.2: Effect of SDS concentration of MWCNT dispersions. Representative SEM of dispersed CNTs after 15 min sonication with surfactant concentration of (a) \& (b) 20 drops $(11.4 \mu \mathrm{M})$, (c) \& (d) 40 drops $(23.1 \mu \mathrm{M})$, (e) \& (f) 80 drops $(46.2 \mu \mathrm{M})$ and (g) \& (h) 120 drops $(69.3 \mu \mathrm{M})$ SDS solutions. The 160 drop $(92.4 \mu \mathrm{M})$ result is not shown because there is no improvement over 120 drop $(69.3 \mu \mathrm{M})$. Circles indicate individual or few MWCNTs. Note that the scale bars vary from image to image. Reprinted with permission from reference [48]. 


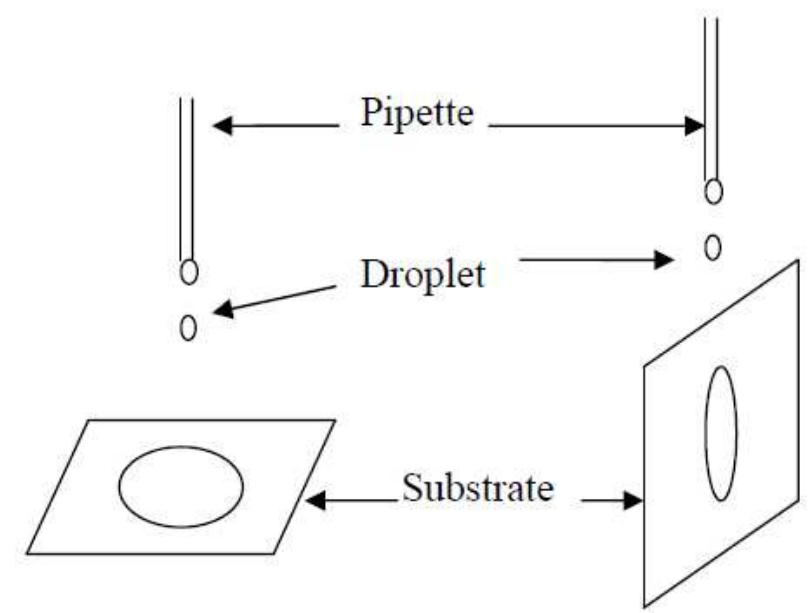

(a) Horizontally

(b) Vertically

Figure 4.3: Comparison of horizontal and vertical MWCNT dispersion methods. Reprinted with permission from reference [48].

used to approximate their density. Lastly, a factor of $1 / 6$ was applied to account for three factors, (1) the space between the VA-MWCNTs, estimated to be $50 \%$ of the total volume, (2) the MWCNTs are only $2 / 3$ graphite by volume because of the inner channel and (3) $1 / 2$ the weight of the MWCNT is actually from the metal catalyst particle. The optimal weight ratio of MWCNT to surfactant is estimated to be 0.3 .

\subsubsection{Effect of Dispersion Method}

In addition to dispersing the MWCNTs onto a horizontal substrate, the effect of what we call vertical dispersion was investigated; the two methods are schematically compared in Figure 4.3. The vertical method utilizes gravity to create fluid flow down the surface of the substrate. The substrate is generally propped up against surface at about $80^{\circ}$ from horizontal as the solution is dispensed onto the substrate. Figure 4.4 shows representative SEM comparing the horizontal and vertical dispersion methods. As before, increasing SDS concentration improves the dispersion, with two differences. First, with increasing SDS the MWCNTs dispersed via the vertical method show improving preferential alignment with the fluid flow direction; it is important to note that this is true toward the top and center of the substrate, but not at the bottom where the majority of the 


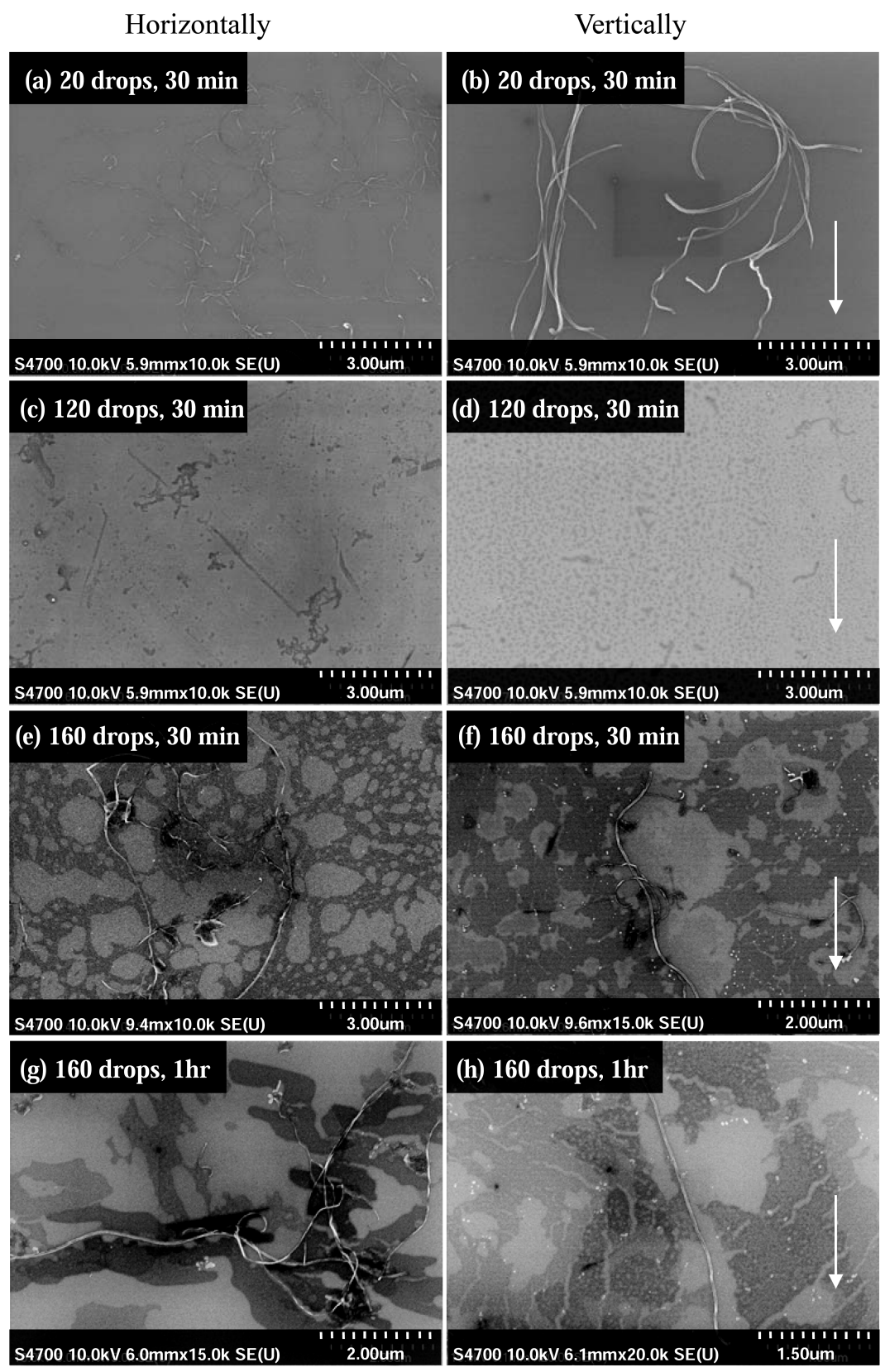

Figure 4.4: Representative SEM comparing horizontal and vertical MWCNT dispersion results. Unlike the horizontal dispersion, increasing the number of drops to 160 leads to better dispersion and enhances preferential alignment. Reprinted with permission from reference [48]. 
solution gathers and the tubes bundle and show no direction preference in that region. Second, it is 160 drops, not 120, that show the best result here. We theorize that the increasing amount of SDS on the substrate surface somehow help the MWCNT to adhere to the substrate and the increased adhesion inhibits curling.

One major problem with this method is that the majority of the solution quickly flows to the bottom of the substrate, and the majority of the CNTs go with it. Therefore, the density of the dispersed CNTs is much lower that the horizontal method. This can be remedied by using successive drops on the substrate, letting the first dry before the next is applied. However, the result is a much larger amount of SDS on the substrate surface. In order to reduce this effect, the substrate is very gently placed in a bath of pure ethanol to remove some of the excess SDS (and a small percentage of MWCNTs). By repeating the drop, dry, bath process, the density of dispersed MWCNTs on the substrate can be tuned.

This method was used along with photolithography to create single or few MWCNT connections between microelectrode pads. Such geometries can be used to measure the properties of the CNTs or make sensor devices. SEM of the results are shown in Figure 4.5. We went on to use this technique to measure the I-V of a single thermal CVD grown MWCNT between patterned glassy carbon electrodes, shown in Figure 4.6. This same method can be applied for measuring any nanomaterial that can span the $2 \mu \mathrm{m}$ gap. Subfigure (D) shows why surfactant-assisted dispersion is undesirable for contaminant-sensitive applications - the I-V shows non-linearity induced by surfactant contamination.

\subsubsection{Surfactant-free Dispersions}

Investigations into the duration of ultrasonication were also carried out. The ultrasonicator directs high intensity ultrasonic energy into the suspension so that the particles in the suspension are vigorously agitated. Whenever the MWCNTs suspension are sonicated, the tubes may break, resulting in shorter tubes. The only trend observed during the SDS experiments is that for different concentrations of surfactant, the optimum sonication time may be different. In general, for the concentrations that we used in our experiments, the optimum sonication duration was below $1 \mathrm{hr}$ (around $30-45$ minutes).

For many applications, such as the molecular electronics devices and sensors, surfactants are 


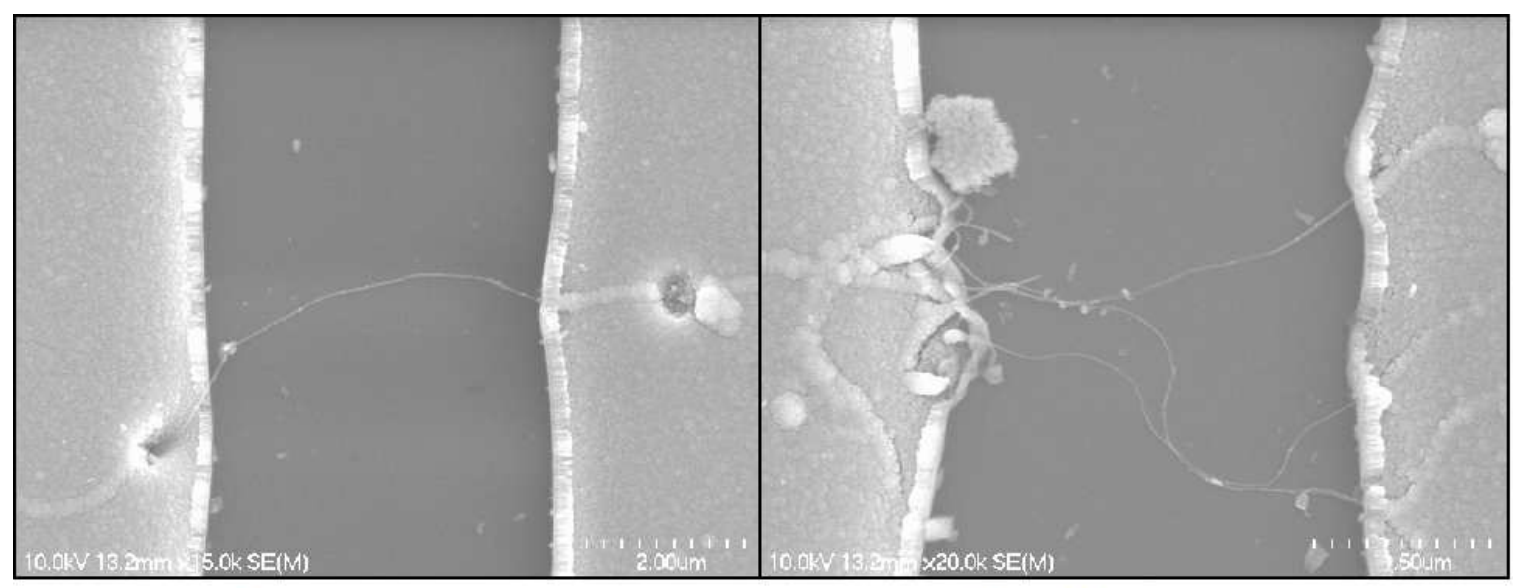

Single connection

Multiple connection

Figure 4.5: Single and few MWCNT connections between photolithographically patterned electrodes made using the vertical dispersion method.

a contaminant that cannot be afforded. While SDS can be removed by annealing, we have no way of testing the effectiveness of the removal or its side effects, such as residual contamination. In addition, having SDS layers on the substrate surface is often not desired since it can reduce metal film adhesion. To this end, a long investigation was undertaken with the goal of optimizing surfactant-free dispersions of thermal CVD MWCNTs.

The ultrasonicating bath used in the SDS experiments seemed unable to achieve our desired result. After a long investigation of various times (up to 6 hours) and MWCNT concentrations, the tubes were always either still tangled, broken into small pieces, or a combination of tangled and broken pieces. We invested in a new sonicator that is placed directly into the solution (Sonics, Vibra Cell), Figure 4.7. At first, it looked to be the same as the sonicating bath, however we discovered that low density, high sonication amplitude and long sonication times yielded the results we were looking for.

For optimal DEP, a solution of $4 \mathrm{~mm}^{2}$ stock MWCNT growth area per $20 \mathrm{~mL}$ ethanol sonicated at $80 \%$ amplitude for 3 hours is desired. The ethanol will evaporate over this period and will need to be refilled every 20 minutes or so, in order to minimize the variation of solution volume. A calculation like the one in section 4.1.3 was done, estimating the concentration of carbon nanotubes to be $0.56 \mathrm{mg} / \mathrm{mL}$. 

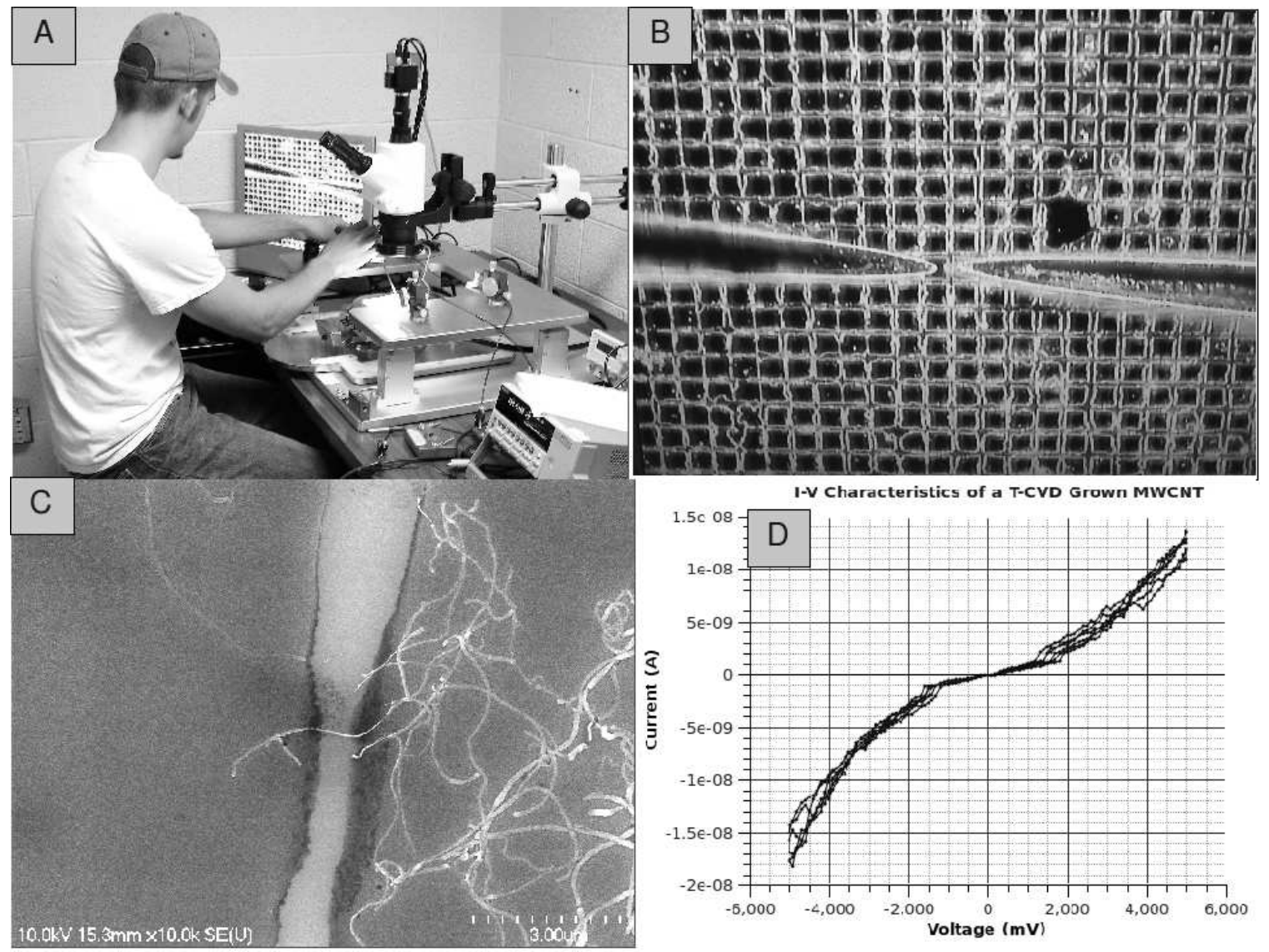

Figure 4.6: I-V characteristics of a TCVD grown MWCNT between two glassy-carbon electrodes. (A) The microprobe station used to make the connections to the microelectrode pads. (B) Optical microscope image from the probe system display showing the array of electrode pads and the probes about to contact the pads. (C) SEM image (taken after measurement) of the tested MWCNT. (D) Current-voltage characteristics of the MWCNTs through multiple current sweeps, showing non-linear response due to surfactant contamination. 


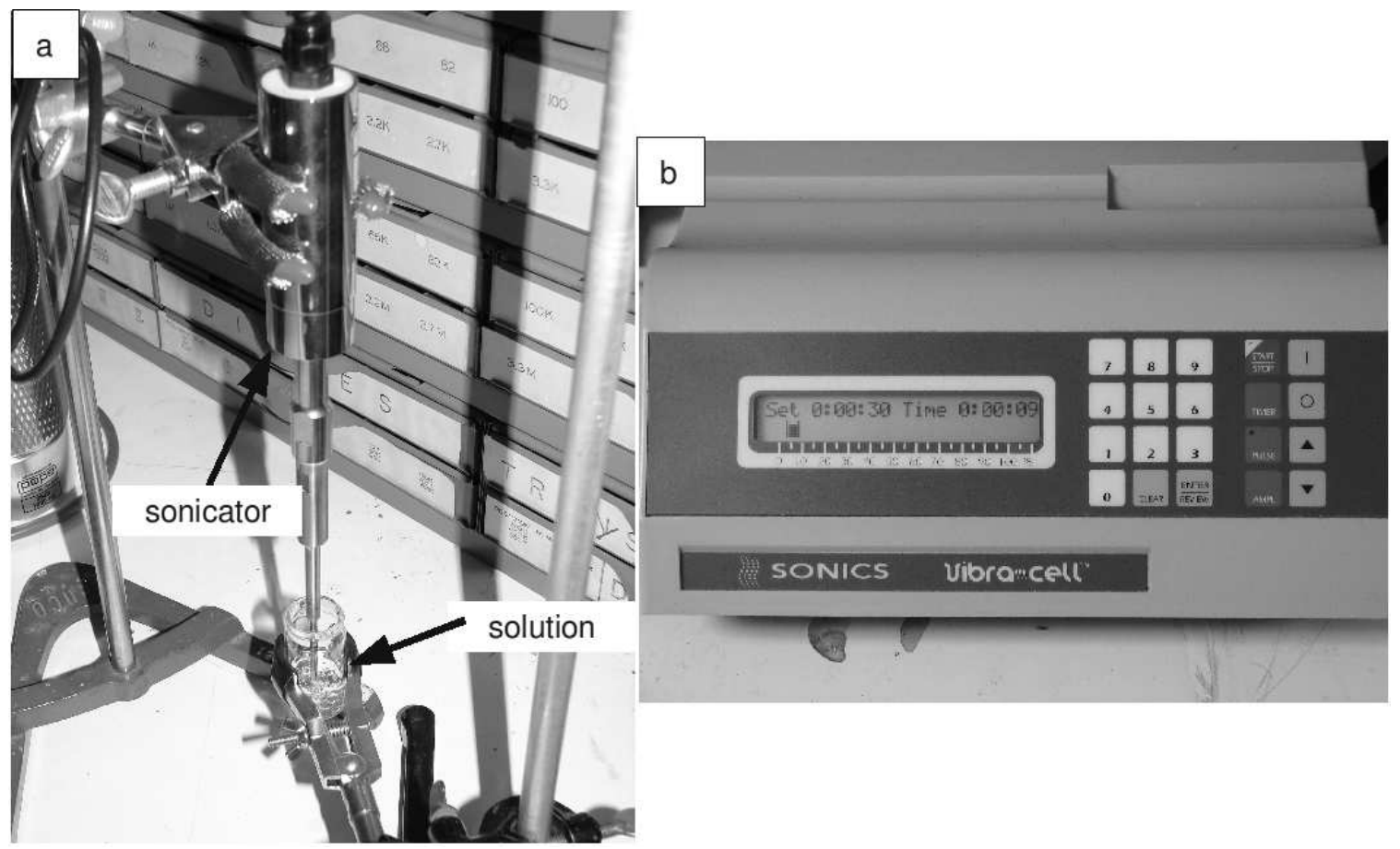

Figure 4.7: (a) The direct sonicator is placed directly within the solution. (b) The controls for the sonicator.

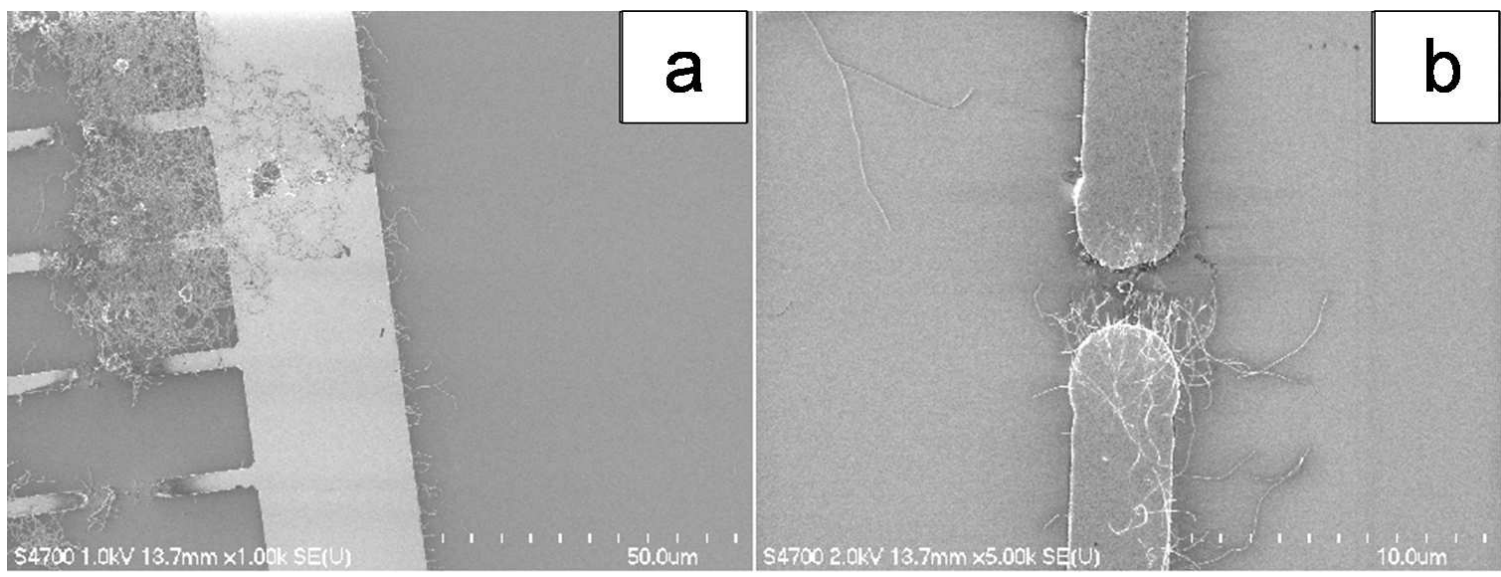

Figure 4.8: (A) At 2.5 hours of sonication and below, the CNTs remain mostly in large clumps. (B) At 3.5 hours and above the majority of tubes are broken into small fragments too small to span the gap. 


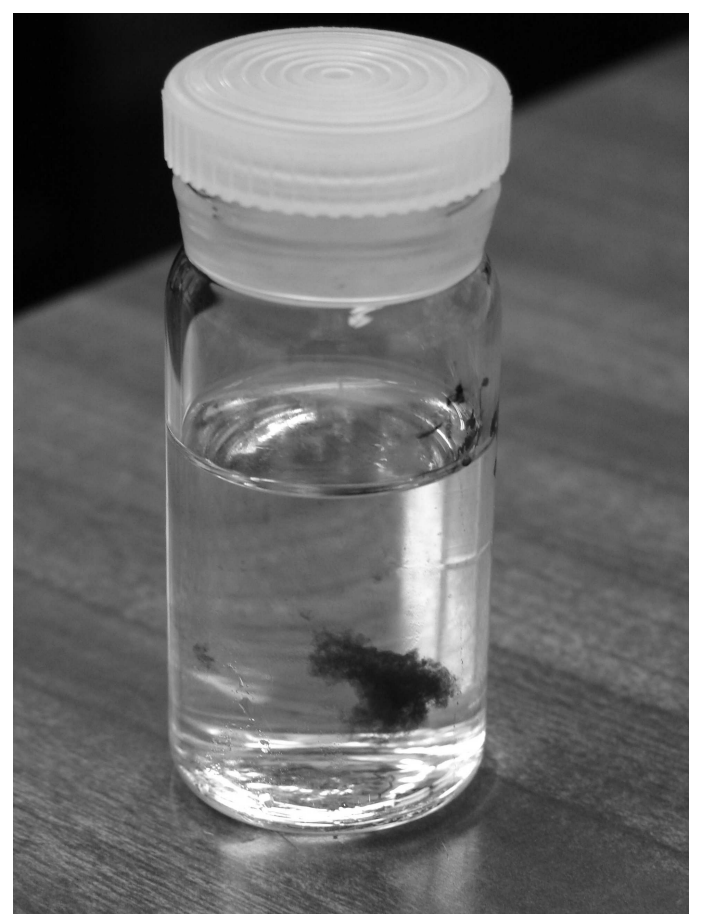

Figure 4.9: Carbon nanotubes in dispersion begin condensing immediately after condensation, and the aggregate becomes visible within 15-20 minutes. 
We found that for times under 3 hours, the MWCNTs are still very tangled and in large clumps, and for 4 hours and above the tubes have broken into pieces too small for use, Figure 4.8.

Of vital importance, because there is no surfactant preventing MWCNT aggregation, the dispersion begins decreasing in quality immediately after the sonication is stopped, Figure 4.9. It is not advisable to resonicate a solution that has been used because it results in breaking the tubes into small pieces. The procedure must be, therefore, to prepare fresh solutions for each experiment, sonicate for 3 hours and disperse immediately. If the solution must be drawn multiple times, the sonication should be immediately restarted in between each drawing.

While there was no time for a formal investigation, it was found that if that the concentration of MWCNTs is increased, the necessary sonication time must be increased. It takes a longer time for the tubes to be shaken out of the larger tangles, and it seems the tubes do not break until they are separated.

It should be noted that this method is very sensitive to contaminants. At one point, the dispersions we made for use in dielectrophoresis were not untangled using our normal CNT concentrations and sonication conditions. After some short investigation, it was discovered that someone had switched our ethanol supply from 200 proof to $95 \%$ ethanol. This difference was enough to stop the process from working.

We chose to focus on ethanol dispersions, but we did investigate water. Unfortunately, because the large amount of time necessary for a drop of water to evaporate during dielectrophoresis, the electrode patterns are subjected large resisitive heating. This heating causes severe damage during dielectrophoresis, as shown in Figure 4.10.

The surfactant-free dispersions of TCVD MWCNTs was absolutely vital to getting single and few MWCNTs between electrodes, and will be utilized for dielectrophoresis in Section 4.2.5, and later in making molecular junctions.

\subsection{Dielectrophoretic Deposition}

\subsubsection{Background Theory}

Many techniques have been used to place CNTs, but each have drawbacks, some of which will be discussed here. Spin coating, for example, is cheap and simple, but lacks control of position and 


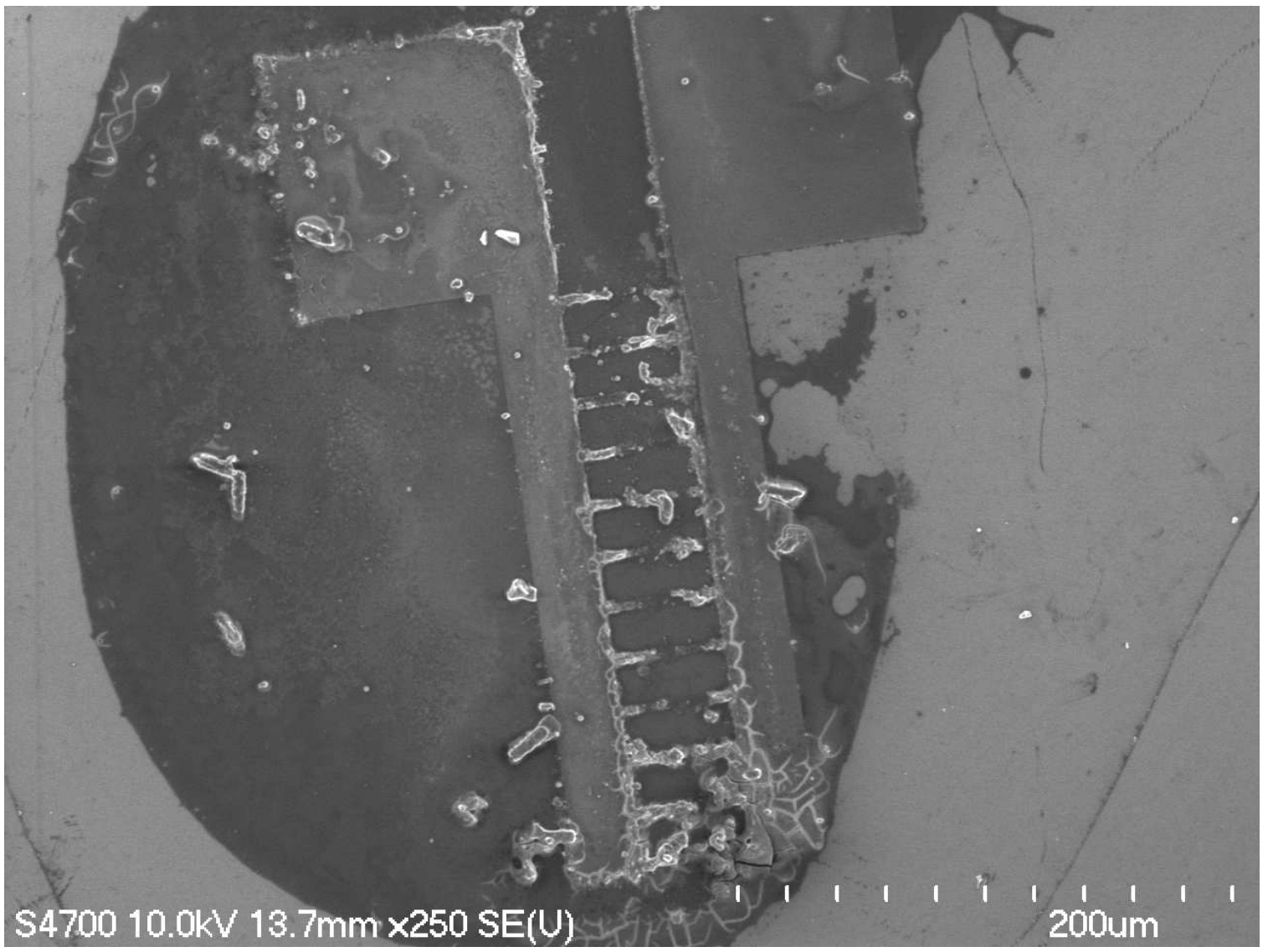

Figure 4.10: Dielectrophoresis in water requires much longer current flow times, and the resistive heating damages and destroys the electrodes. 
distribution $[18,93]$. Direct growth of CNTs requires high temperatures and has the additional disadvantages of poor selectivity and non-compatibility [94, 95]. Manual attachment allows precise placement, but is unviable for devices of any significant density due to the enormous amounts of time necessary to place individual CNTs. Dielectrophoresis (DEP), on the other hand, is quick, cheap and can result in well-aligned CNTs in the position of interest. Of extreme importance to any of these placement techniques is the ability to create suspensions of CNTs in liquids, which is difficult due to their general low solubility and tendency to aggregate. This chapter will address MWCNT suspensions and their use in dielectrophoresis.

Whenever a polarizable object is placed within an electric field, charges are induced on the material, with a positive charge on one side and an a negative charge on the reverse side. The electric field will act to pull the positive charge in one direction and the negative charge in the reverse direction. In the case of a uniform electric field, these two forces are balanced and the object experiences no net force. However, if the field is non-uniform, the forces will be out of balance and the object will experience a net force [96].

In order to get a feel for how this happens, let us first consider a simple system consisting of a particle in an electric field, with the assumption that the particle's presence does not influence the field. As in Figure 4.11, the negative and positive portions of the dipole are separated by a vector $\vec{d}$ and located by the position vectors $\vec{r}$, and $\vec{r}+\vec{d}$, respectively. This dipole is described by [96, 97]:

$$
\vec{p}=q \vec{d}
$$

The force enacted on the dipole by the electric field is given by:

$$
\vec{F}(\vec{r})=q \vec{E}(\vec{r}+\vec{d})-q \vec{E}(\vec{r})
$$

Here is commonly enacted the dipole approximation by simply assuming that $|\vec{d}|$ is much less than the characteristic dimension of the field uniformity, allowing the expansion [97]:

$$
\vec{E}(\vec{r}+\vec{d})=\vec{E}(\vec{r})+\vec{d} \cdot \nabla \vec{E}(\vec{r})+O\left(d^{2}\right)+O\left(d^{3}\right)+\ldots
$$




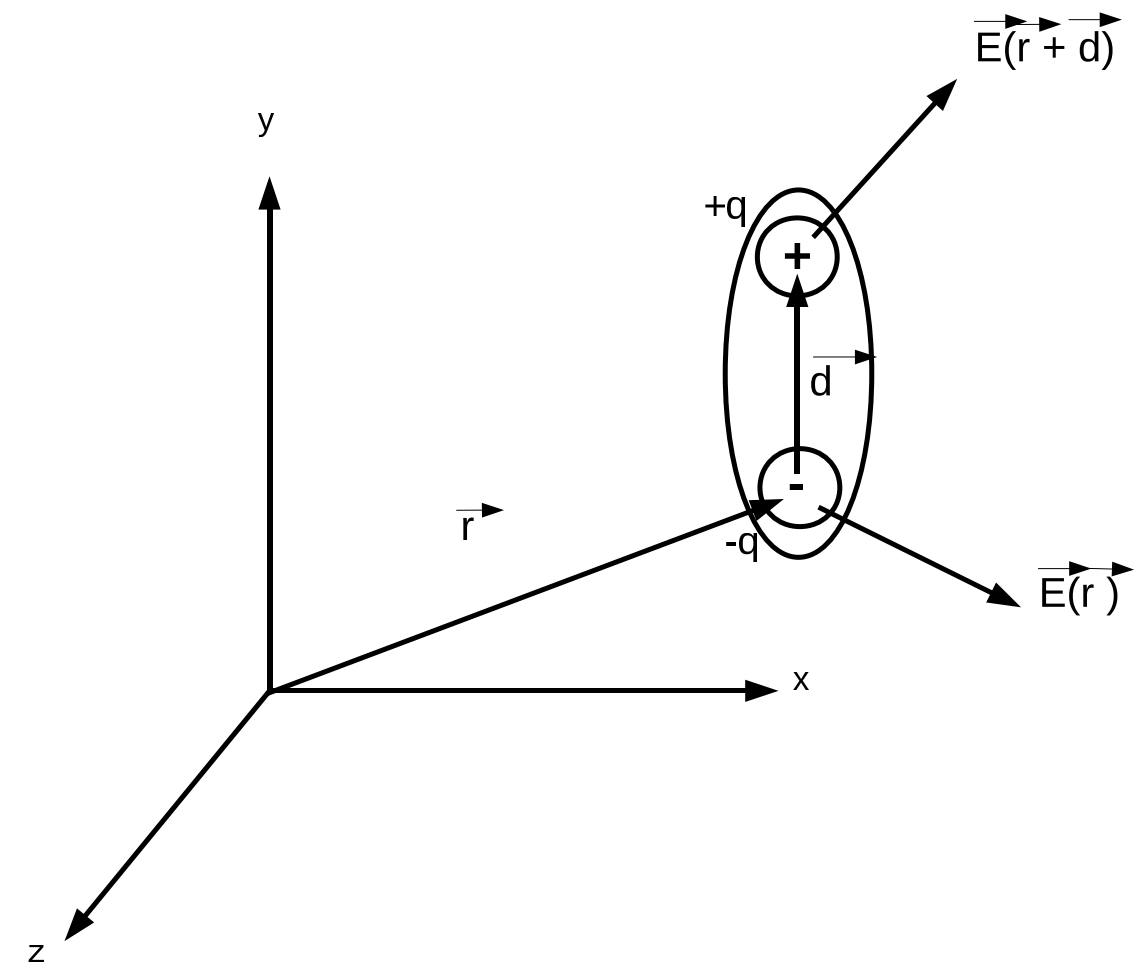

Figure 4.11: A dipole in a non-uniform electric field, showing the vectors and labeling.

$$
\vec{F}(\vec{r})=q[\vec{E}(\vec{r})+\vec{d} \cdot \nabla \vec{E}(\vec{r})]-q \vec{E}(\vec{r})+O\left(d^{2}\right)+O\left(d^{3}\right)+\ldots
$$

If the limit $|\vec{d}| \rightarrow 0$ is taken such that Equation 4.1 remains finite, we can drop all terms of order $d^{2}$ and above, which yields the familiar expression of the force on a dipole [97]:

$$
\vec{F}_{\text {dipole }}=\vec{F}_{d}=q \vec{d} \cdot \nabla \vec{E}(\vec{r})=\vec{p} \cdot \nabla \vec{E}(\vec{r})
$$

As we can see in this simple system, the dipole experiences no force unless the electric field is non-uniform. Further, since strength of the induced dipole is proportional to the strength of the externally applied electric field, the force is a function of $\nabla E^{2}$ and, therefore, is independent of field direction and can be driven by either DC or AC fields [98].

A more complicated model is necessary to consider non-point objects, like carbon nanotubes, in a medium. A common approach is to model the CNTs as slender prolate ellipsoids and to use 
the Rayleigh electrostatics approximation [99]; the key assumptions are that the CNTs are small compared to the spatial change of the electric field $\left(|\nabla \vec{E}|>>l_{c n t}|\nabla \nabla \vec{E}|\right.$, where $l_{\text {cnt }}$ is the length of the CNT), and that the induced field internal to the CNTs is uniform [98]. The dipole moment of the CNT can be determined using [97, 98, 99]:

$$
p_{c n t}=V_{c n t} \epsilon_{m} \vec{E} \cdot \vec{P}
$$

where $V_{c n t}$ is the volume of the CNT, $\epsilon_{m}$ is the permittivity of the medium, $\vec{P}=\operatorname{Re}\left[K^{\|}\right] \vec{u} \vec{u}+$ $\operatorname{Re}\left[K^{\perp}\right](\vec{I}-\vec{u} \vec{u})$ is the polarizability tensor, $\vec{u}$ is the unit vector along the CNT axis and $\operatorname{Re}\left[K^{\|, \perp}\right]$ is the real parts of the radial (or perpendicular) and axial (or parallel) Clausius-Mossotti factors [98]. The Clausius-Mossotti (CM) factors depend on the differences between the complex permitivities of particle material and the medium are given by [98]:

$$
K^{\|, \perp}=\frac{\epsilon_{c n t}^{*}-\epsilon_{m}^{*}}{\epsilon_{m}^{*}+\left(\epsilon_{c n t}^{*}-\epsilon_{m}^{*}\right) L^{\|, \perp}}
$$

where $L^{\|, \perp}$ are depolarization factors dependent on geometry. Carbon nanotubes are generally modeled geometrically to be prolate ellipsoids with high aspect ratios, so for this case the factors are written as [98]:

$$
L^{\|}=\frac{4 r_{c n t}^{2}}{l_{c n t}^{2}}\left(\log \left[\frac{l_{c n t}}{r_{c n t}}\right]-1\right) \quad L^{\perp}=\frac{1-L^{\|}}{2}
$$

where $r_{c n t}$ is the $\mathrm{CNT}$ radius. As expected, the CNTs are more polarizable along the longer axis of the ellipsoid. The permittivities (dielectric functions) of the materials are frequency-dependent in AC fields because the charges must move and dissipate energy; this is written as [98, 100]:

$$
\epsilon^{*}(\omega)=\epsilon-i \frac{\sigma}{\omega}
$$

where $\omega=2 \pi f$ is the angular frequency of the AC field. The CNTs are assumed to be a homogeneous material with the same $\epsilon_{c n t}$ and $\sigma_{c n t}$ for both CM factors because the anisotropy in the dielectric constants is negligible [98]. Further, $\epsilon_{c n t}$ and $\sigma_{c n t}$ are treated as being independent of frequency, since the values should only begin to deviate from their DC values in the Terahertz range [101].

Very importantly, the Clausius-Mossotti factors in Equation 4.7 have frequency dependence in 
both magnitude and phase. The phase angle represents a lag in the induced dipole relative to the changing external field due to a finite charging time [98]. The build-up and decay time are expressed by the Maxwell-Wagner relaxation time constant [97]:

$$
\begin{aligned}
\tau^{\|, \perp} & =\frac{\left(1-L^{\|, \perp}\right) \epsilon_{m}+L^{\|, \perp} \epsilon_{c n t}}{\left(1-L^{\|, \perp}\right) \sigma_{m}+L^{\|, \perp} \sigma_{c n t}} \\
\omega^{C} & =\frac{1}{\tau^{\|}}
\end{aligned}
$$

Here $\omega^{C}$ represents the critical frequency at which the charge within the medium can no longer keep up with the switching of the AC field and beyond which the dipole moment is reduced; for carbon nanotubes, the critical frequency of the axial direction is what matters. Note that is is possible for the $\mathrm{CM}$ factors to be or become negative in certain situations. If conditions are such that the particle is more polarizable than the medium, the DEP force pushes the particle to regions of higher electric fields in a process called positive dielectrophoresis. If the medium is more polarizable, the particle is pushed to the weaker regions of electric field; this is called negative dielectrophoresis [96].

We can consider time-averaged force on the carbon nanotubes as well. Again, in a non-uniform $\vec{E}$, the force on the induced dipole is give by [96]:

$$
\vec{F}=(\vec{p} \cdot \vec{\nabla}) \vec{E}
$$

In this case, the time-averaged force on a tube aligned with the field lines can be given by [100]:

$$
\left\langle F_{D E P}\right\rangle=\frac{1}{2} \pi r_{c n t}^{2} l_{c n t} \epsilon_{m} R e\left(K_{\|}\right) \nabla|E|^{2}
$$

This expression makes it explicit that the force the CNT feels should be proportional to the strength of the field and the CM factor and, therefore, has a dependence on frequency.

Another effect the that must be considered is the torque caused by the electric field that rotates the CNTs and aligns them with the field. The torque can be expressed as [102]:

$$
\tau=\vec{p} \times \vec{E}=l_{\text {cnt }} q E \sin \theta
$$


where $\theta$ is the angle between the CNT axis and the direction of $\vec{E}$ [102].

The time averaged torque is given by [100]:

$$
\langle\tau\rangle=\frac{1}{6} \pi r_{c n t}^{2} l_{c n t} \epsilon_{m} E^{2} \sin \theta \cos \theta \operatorname{Re}\left(K_{\|} K_{\perp}\right)
$$

\subsubsection{Research Goals and Collaborators}

The research goals for dielectrophoresis were:

- Develop and refine the capability to perform dielectrophoresis with carbon nanotubes beginning with simple planar gap electrodes. This included patterned metal electrode deposition (PLD, plasma sputtering, shadow masks, FIB cuts, photolithography mask development), probe station and electronics set-up, and study of MWCNT dispersions.

- Understand the influence of the relevant parameters, namely: medium, AC field strength and AC field frequency.

- Use dielectrophoresis to create clean MWCNT connections between microelectrodes.

- Use dielectrophoresis to create a MWCNT cross in order to fabricate molecular electronic devices (discussed in following chapters).

Initial work (up through planar gaps) was done alongside Vijaya Kayastha. Many times during the course of the research, we conferred with Libao An to compare notes and discuss ideas.

Our beginning point was the work has done on by Yamamoto et al., Reference [103]. They found that for $\mathrm{AC}$ electric fields from $10 \mathrm{~Hz}-10 \mathrm{MHz}$, MWCNTs (length 1-5 $\mu \mathrm{m}$, diameter 5-20 nm) dispersed in IPA were attracted and aligned between $\mathrm{Al}$ electrodes with $400 \mu \mathrm{m}$ gaps. The degree of alignment was found to increase with both MWCNT length and ac frequency. Graphite impurities had a different response, and this could be used for purification [103].

Our research aims to optimize DEP deposition of MWCNTs for use in making CNT-based devices. This entails optimization of CNT growth, dispersion, AC DEP parameters (field strength, frequency) and electrode design and fabrication. In particular, the Clausius-Mossotti factors relate to the dielectrophoretic force by Equation 4.13 and are frequency dependent, so dependence on field strength and frequency are expected. More specifically, for carbon nanotubes the theory predicts that as frequency increases, the CM factors (and the force) will be reduced above a critical frequency $[98,100,104]$. An example of such a prediction for spherical particles $\left(\epsilon_{p}=8.854 \times 10^{-8} \mathrm{~F} / \mathrm{m}\right.$, 


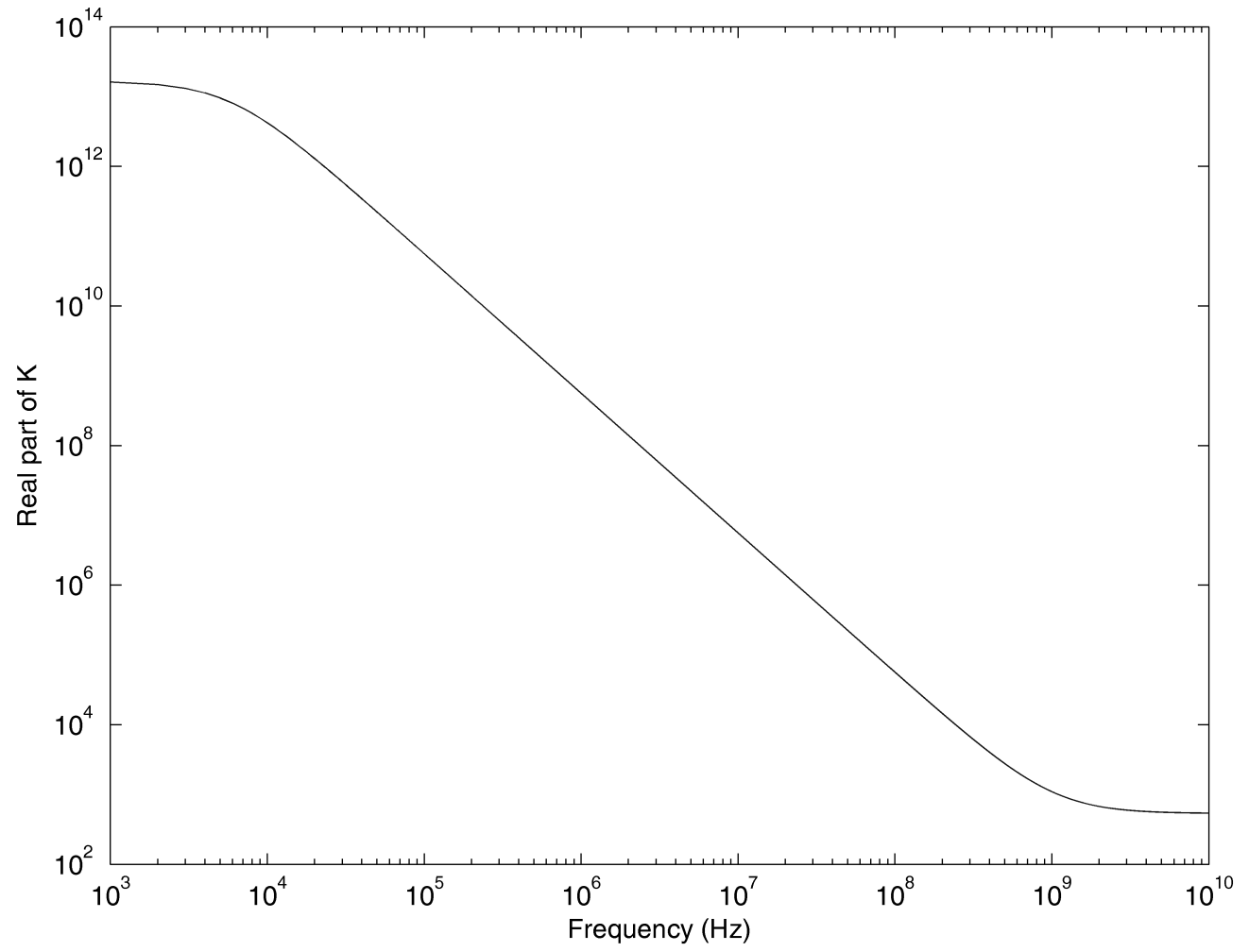

Figure 4.12: Example plot of the real part of the Clausius-Mossotti factor for a spherical metallic particle.

$\epsilon_{m}=1.647 \times 10^{-10} \mathrm{~F} / \mathrm{m}, \sigma_{p}=1 \times 10^{8} \mathrm{~S} / \mathrm{m}$ and $\left.\sigma_{m}=6 \times 10^{-6} \mathrm{~S} / \mathrm{m}\right)$ is shown in Figure 4.12. However, the approximations that theory is predicated on are, by the definition of our experimental system, not true. The question then is: will the predictions still hold? If so, we can use them to tune our deposition density.

The initial DEP experiments took place using a WAVETEK Sweep Generator model 22 as the AC electric field source. This was connected to two tungsten microprobes used to contact the electrodes. The fields were always turned on before the drop of suspension was placed on the gap, and were turned off right after the drop of solution completely dried. All experiments were conducted using the stock thermal and plasma-enhanced MWCNTs grown in-house and described earlier in this dissertation. The results were studied using a Hitachi S4700 field-emission scanning electron microscope (FESEM). 


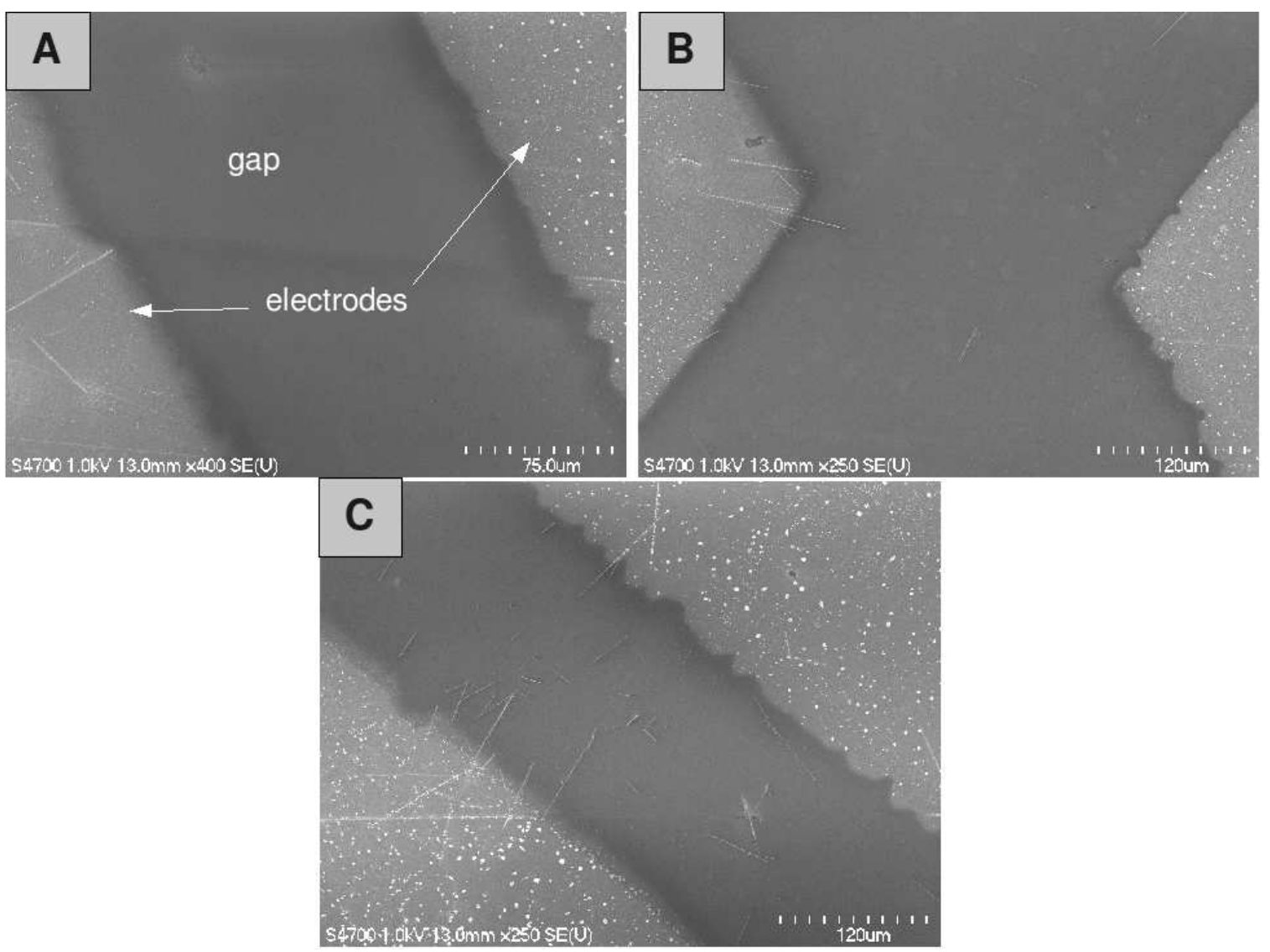

Figure 4.13: SEM images of initial electrode gaps made by PLD shadowmasking. ZnO nanotubes were found to prefer alignment parallel or perpendicular to the field.

\subsubsection{Initial DEP Experiments}

The very first experiments, conducted with Vijaya Kayastha, were simple proof-of-concept attempts and at the time we had no photolithographic capability. Electrodes and gaps were made by depositing metal by PLD with one or more optical fibers serving as shadowmasks to form the gaps. We found we could make electrically isolated iron pads and attempt dielectrophoretic deposition. Figure 4.13 shows some of the initial results with $\mathrm{ZnO}$ nanotubes. We did observe some preferential alignment, mostly parallel to the field or perpendicular to it.

The gaps were much larger than desired, at $120 \mu \mathrm{m}$, but we discovered ways of reducing using materials available in the lab, ultimately by using human hair, down to about $60 \mu \mathrm{m}$. Both $\mathrm{Si} / \mathrm{SiO}_{2}$ and glass substrates were shown to work. 


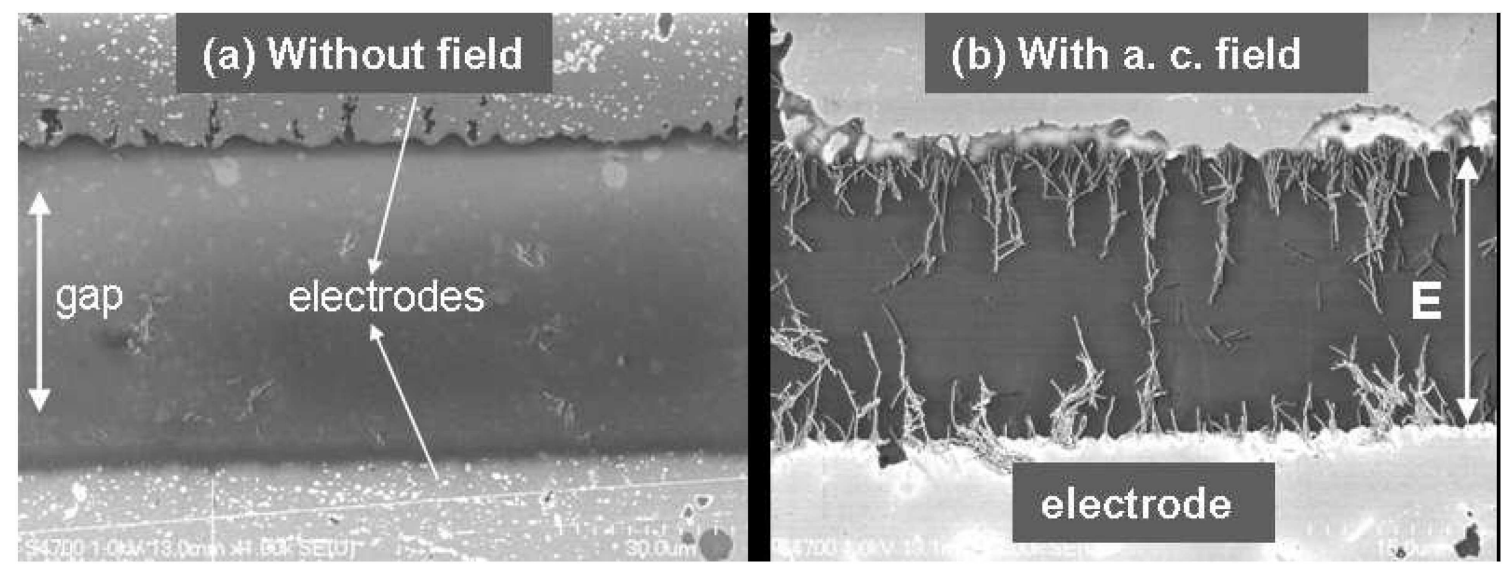

Figure 4.14: Comparison of CNT deposition in a flat electrode gap with and without applied AC electric field. Reprinted (with author's rights) from Reference [105].

We were able to show using shadowmask gaps that the AC field is responsible for alignment of the CNTs. This was done by comparison of tubes in the gap deposited without and with a $10 \mathrm{~V}$ peak to peak $\left(\mathrm{V}_{p p}\right), 5 \mathrm{MHz}$ electric field. Figure 4.14 shows the difference: without field the tubes are randomly placed and with field they are well oriented in the direction of the field and contacting the electrodes. In addition, the MWCNTs are capable of forming chains by attaching tail-to-tip once one tube has contacted the electrode.

\subsubsection{Flat Electrode Gaps}

\section{Experiments}

Once we gained the ability to use the focused ion beam (FIB) to make cuts, the first precise "flat gap" experiments were done along with Vijaya Kayastha; here was a simple electrode configuration of a uniform gap between two flat Fe electrodes. Electrodes were patterned on glass substrates by pulsed laser deposition of an iron film to a thickness of $50 \mathrm{~nm}$. The gaps of roughly $1 \mu \mathrm{m}$ were formed by focused ion beam (FIB) milling. The CNT dispersion was made from MWCNTs grown by a dual plasma-enhanced chemical vapor deposition (PECVD) process [106],grown directly on a silicon substrate. These MWCNTs had lengths and widths of roughly $2 \mu \mathrm{m}$ and $50 \mathrm{~nm}$, respectively. After growth, the $7 \mathrm{~mm}$ diameter growth region was removed and was placed in ethanol for dispersion to a density estimated to be $10^{8}$ MWCNTs per mL. The suspension was placed in an ultrasonicating 
bath for 2 hours to facilitate dispersion.

Before deposition, the electrodes were connected by a microprobe station to a WAVETEK Sweep Generator (model 22), and the AC field was turned on. Next, a $40 \mu \mathrm{L}$ drop of the ethanol/CNT dispersion was applied directly to the gap giving it time to completely dry before the applied field is turned off.

In order to observe the effect of applied field strength, the frequency was fixed to $2 \mathrm{MHz}$ while varying the voltage from $3-20 \mathrm{~V}_{\text {peak-to-peak }}\left(\mathrm{V}_{p p}\right)$; Figure 4.15 shows the results of this test. Clearly the density (number of CNTs per unit length of the electrode gap) of tubes increases along with increasing field strength. Alignment is generally good with no apparent dependence on the field strength. This is, therefore, one method of controlling the spacing between CNTs, which may be important for fabrication of density-controlled parallel nanoelectronic devices.

Next, the voltage was held at $20 \mathrm{~V}_{p p}$ and the frequency varied in order to study the effects of $\mathrm{AC}$ frequency on DEP; the results are shown in Figure 4.16. Low frequencies (50 Hz) showed many CNTs and contaminants clumping in the gap, while a few span the gap with little to no alignment. Increasing the field to $1 \mathrm{MHz}$ showed increased alignment; most tube bundles show better alignment than the clumps seen at $50 \mathrm{~Hz}$. At $5 \mathrm{MHz}$ the CNTs show alignment, but a remarkably lower density; the bundles of tubes are farther apart and contain fewer tubes per bundle. The density is further reduced at $10 \mathrm{MHz}$. This shows that the degree of alignment and CNT density can be tuned by controlling the AC frequency of the applied field.

All results were reproduced and confirmed by DEP with CNTs grown by thermal CVD processes as well. We were able to confirm that the TCVD tubes retained the tendency of the deposition density to decrease once the AC frequency has passed a critical frequency, as shown in Figure 4.17. Here a $20 \mathrm{~V}_{p p}$ field was used and clearly shows lower density at $5 \mathrm{MHz}$ than $3 \mathrm{MHz}$.

\section{Analysis}

The basic mechanism for the dielectrophoretic deposition is simple, inhomogeneous fields bring about nanotube migration toward the regions where the field is the highest: between the electrodes. Once a tube touches an electrode, it acquires the potential of the electrode and can attract CNTs to itself, leading to the chains observed by the SEM. Since higher electric fields result in higher field-induced forces on the CNTs, higher AC voltage yields a higher density of oriented tubes. 

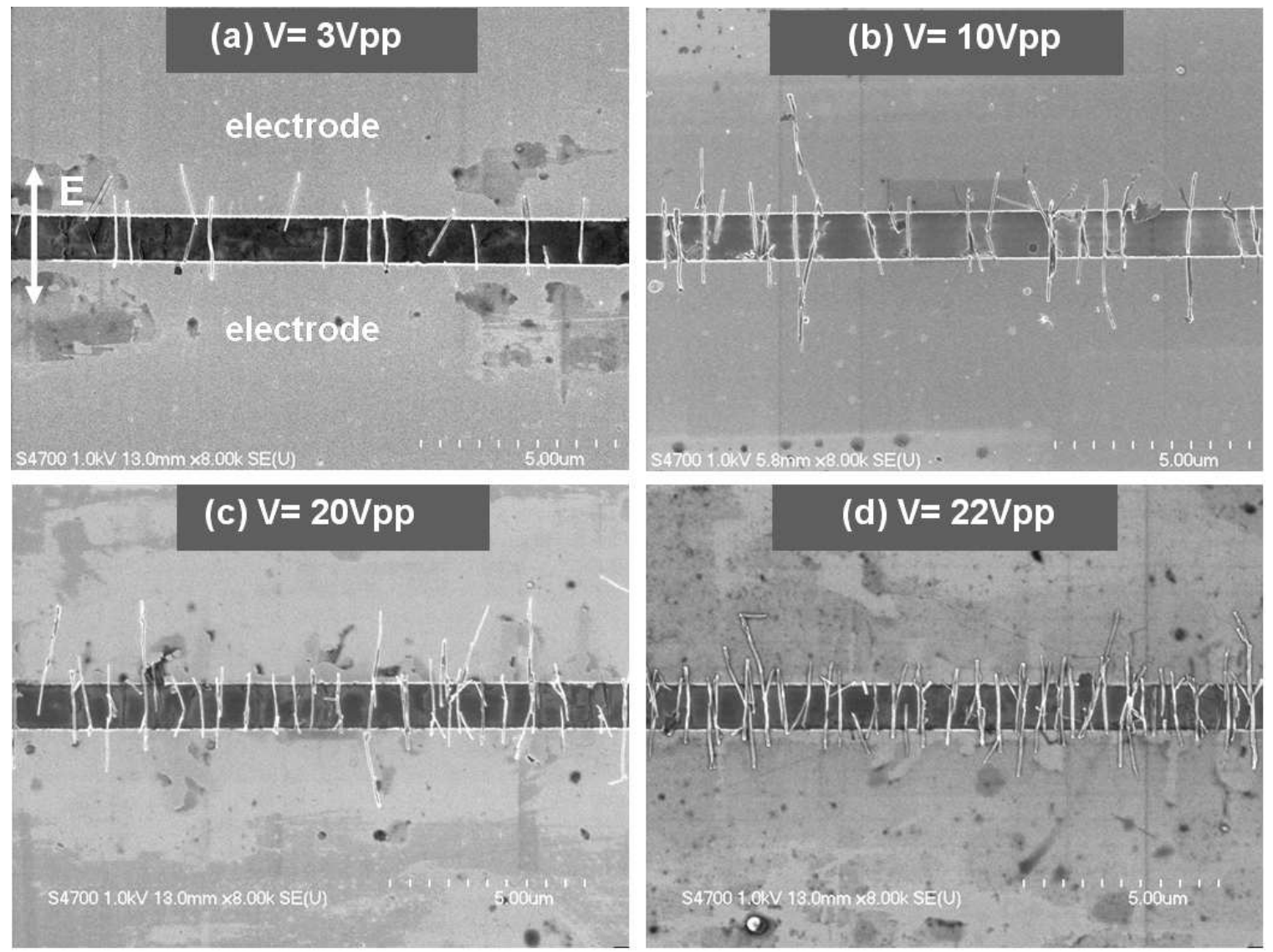

Figure 4.15: Effect of varying AC voltage on CNT deposition. Frequency was kept fixed at $2 \mathrm{MHz}$. As field strength increases from (a) $3 \mathrm{~V}_{p p}$ to (d) $22 \mathrm{~V}_{p p}$, the density of deposited tubes increases. 

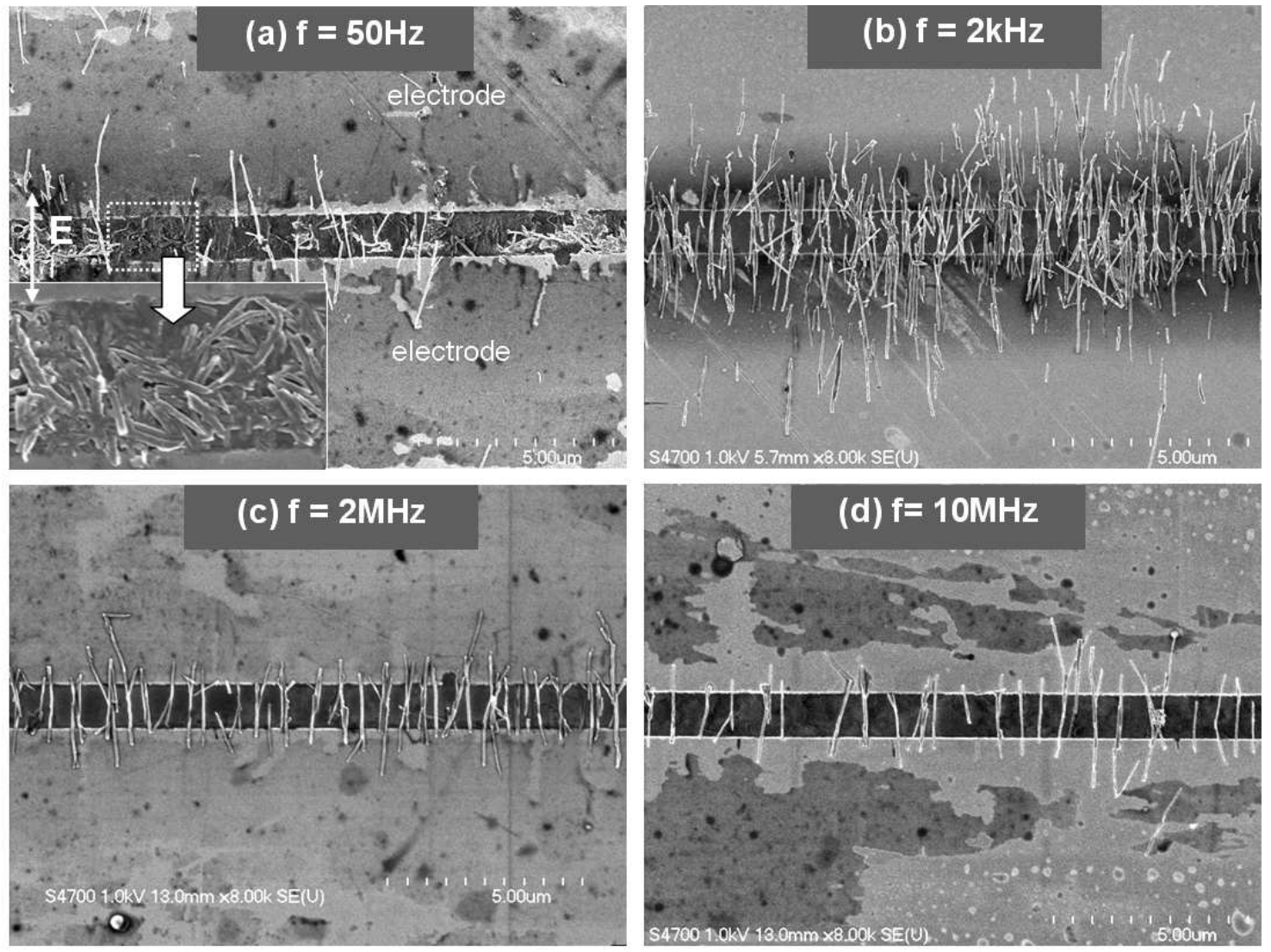

Figure 4.16: CNT deposition with varying AC frequency. Voltage was kept fixed at $20 \mathrm{~V}_{p p}$.(a) 50 $\mathrm{Hz}$ is dirty and random in alignment and placement with very few tubes spanning the gap. (b) 2 $\mathrm{kHz}$ shows an extremely high tube density and alignment, while the contamination appears to be gone. At (c) $2 \mathrm{MHz}$, the density of tubes is much lower, and continues to fall at (d) $10 \mathrm{MHz}$. 

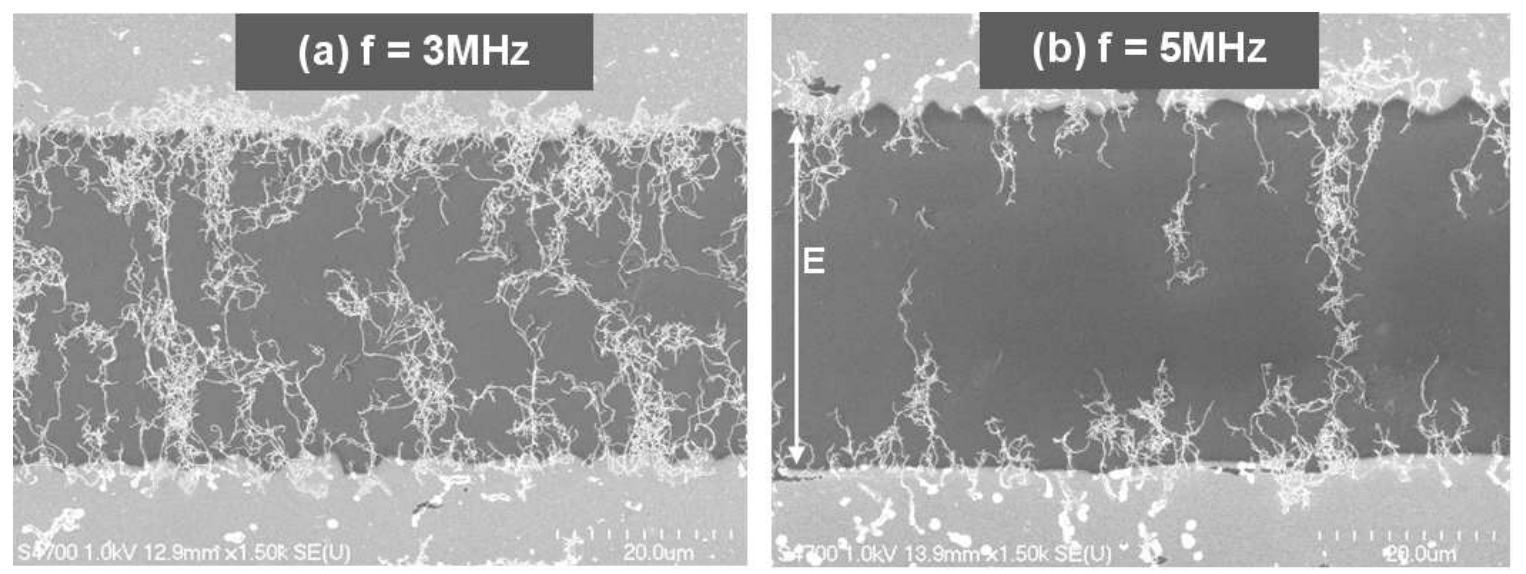

Figure 4.17: Effect of varying the frequency on thermal CVD grown MWCNTs. Voltage was fixed at $20 \mathrm{~V}_{p p}$. The same reduction in density with increasing frequency is seen to take place with TCVD tubes.

However, the relationship is more difficult with frequency. It is clear that deposited CNT density rises, peaks and then continues falling with as frequency increases. If we look to the theory described earlier, the Clausius-Mossotti factor does indicate there should be a fall as frequency increases. This occurs because the response of the electrons in the CNTs can no longer keep up with the alternating AC field and, as a result, the induced dipole moment shrinks. Smaller induced dipole means lower force, so the CNTs do not have time to reach the gap in the numbers they could before the critical frequency.

A calculation for the $\mathrm{CM}$ factor and $\omega_{C}$ was carried out using the following values: $\epsilon_{c n t}=$ $8.854 \times 10^{-8} \mathrm{~F} / \mathrm{m}[107], \epsilon_{\text {ethanol }}=2.1516 \times 10^{-10} \mathrm{~F} / \mathrm{m}[108], \sigma_{c n t}=2.221 \times 10^{4} \mathrm{~S} / \mathrm{m}[109]$ and $\sigma_{\text {ethanol }}=1.3 \times 10^{-7} \mathrm{~S} / \mathrm{m}[110]$, with dimensions equal to the TCVD grown MWCNTs, $r=0.02 \mu \mathrm{m}$ and $l=13 \mu \mathrm{m}$. The resulting curve of the real part of the CM factor is shown in Figure 4.18. Recalling that the $\mathrm{CM}$ factor is proportional to the dielectrophoretic force, we can see that, quantitatively, the force does not change appreciably over the frequency range investigated. It is clear that while the CM theory's qualitative prediction is correct, the quantitative results are not accurate. Of course, this makes sense because, as mentioned earlier, the assumptions within the CM theory are explicitly violated by the conditions of our DEP experiments. The model also does not account for there being multiple dipoles in close proximity. There is also experimental evidence that the process is influenced by the substrate, and that the for dispersions without surfactant, solvated molecules affect 


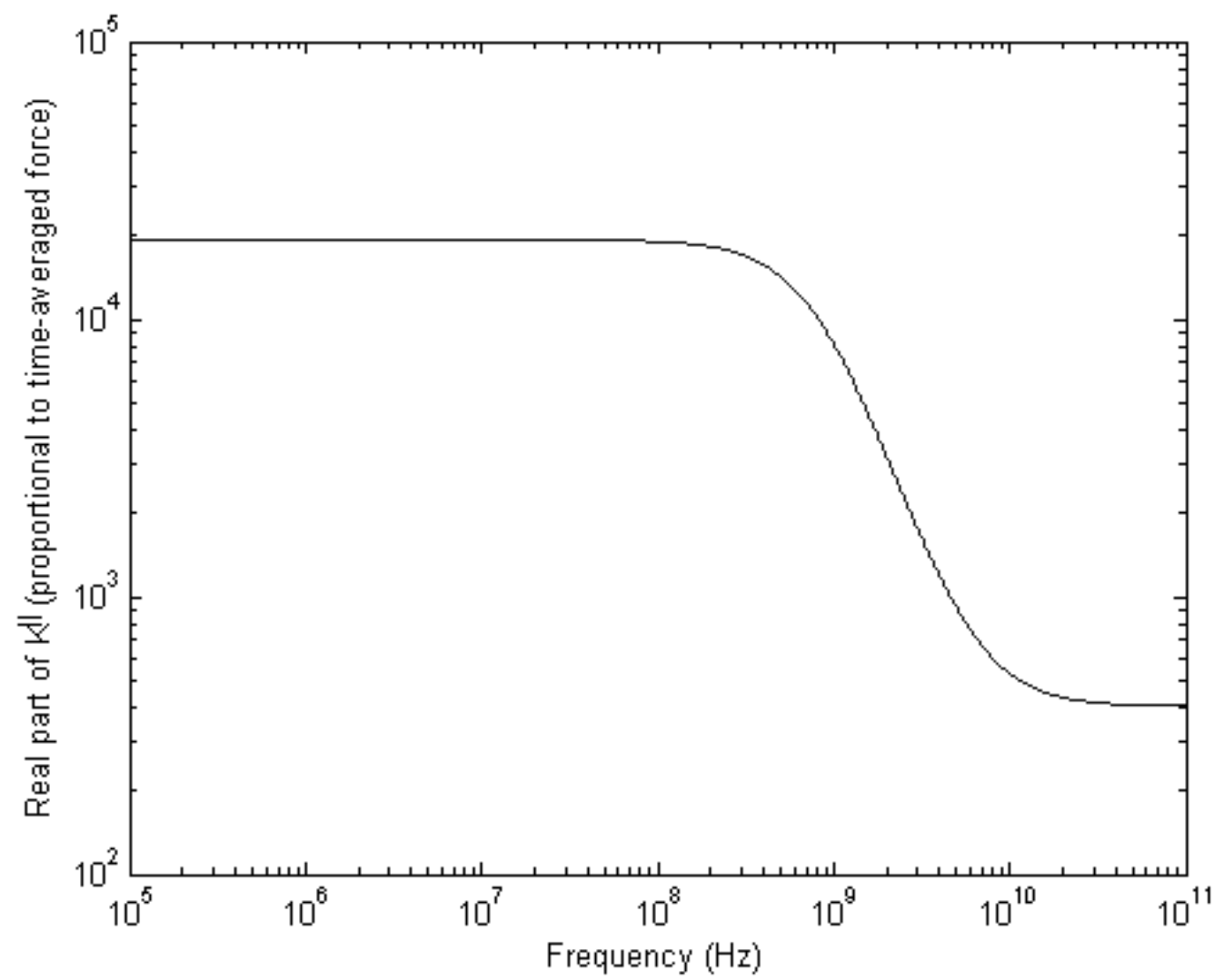

Figure 4.18: The Clausius-Mossotti factor's dependence on frequency for stock TCVD MWCNTs in ethanol. The calculated critical frequency is well outside the experimental range.

the permittivity of the CNTs [111]. There are many layers still not understood or properly modeled.

Yet fundamentally, the mechanism still makes sense for our system, that the frequencies become too high for the electrons to keep up with the switching, resulting in a reduced dipole moment and reduced force. The complication is that there are more parameters than the model takes into account, so we have no predictive power as to what frequency ranges will give us tunability over deposition density. We cannot assume it will be the same for different geometries, we have to test it again for the microelectrode fingers.

We have accounted for the drop in density as frequency increases, but what about the poor alignment and lower density at low frequency? These can be explained by by considering an electrical double layer forming near the electrode surfaces. As a voltage is applied, ions and molecules with 
induced dipoles move to the surface of the electrode. Specifically adsorbed particles form what is referred to as the inner Helmholtz plane on the electrode surface [112]. Beyond the inner Helmholtz plane is a layer of diffuse attracted molecules called the outer Helmholtz plane [112]. If a double layer is given time to form, it can screen nearly all of the applied voltage $[112,113,114]$. Importantly, formation of the double layer takes time; the effect we see is due to this finite charging time. When the switching of electrode polarity is very slow, such as in the $\mathrm{Hz}$ range, the double layer has time to form. As the AC frequency increases, the double layer becomes less and less well formed until we reach a frequency beyond which it has no time to form and no longer screens the electric field $[112,113,114]$; from this point on the effect of the Clausius-Mossotti factor dominates the deposition density. We expect to see this effect again when we move on to the microelectrode finger geometries.

The last observed phenomenon requiring explanation is that of frequency dependent contamination. At $50 \mathrm{~Hz}$ it is clear there are large amounts of impurities gathered in the gap and coating some of the CNTs, but this effect is notably absent at higher field frequencies. The answer to this is related to the Clausius-Mossotti factors for the contaminant particles. While the MWCNTs we consider always have positive CM factors because they are so much more polarizable than the solvent they are deposited in, this is not necessarily the case for other particles. It is known that many particles, particularly small, less conductive ones, have CM factors which switch sign as frequency increases. This critical point occurs when the particles become less polarized than the solution and begin to experience negative dielectrophoresis. Therefore, at higher frequencies the impurities move to the areas of lowest field strength, leaving the areas of interest clean.

We have now explained the observed effects of AC field strength and AC field frequency.

\subsubsection{Microelectrode Fingers}

Because of the mismatch between experimental observation and theoretical prediction, and the fact that model doesn't account for all parameters, a major question still remains: will we retain the ability to tune the density of deposited MWCNTs within the same frequency range when the geometry is changed from simple planar electrodes to more geometrically complex microelectrode fingers? 


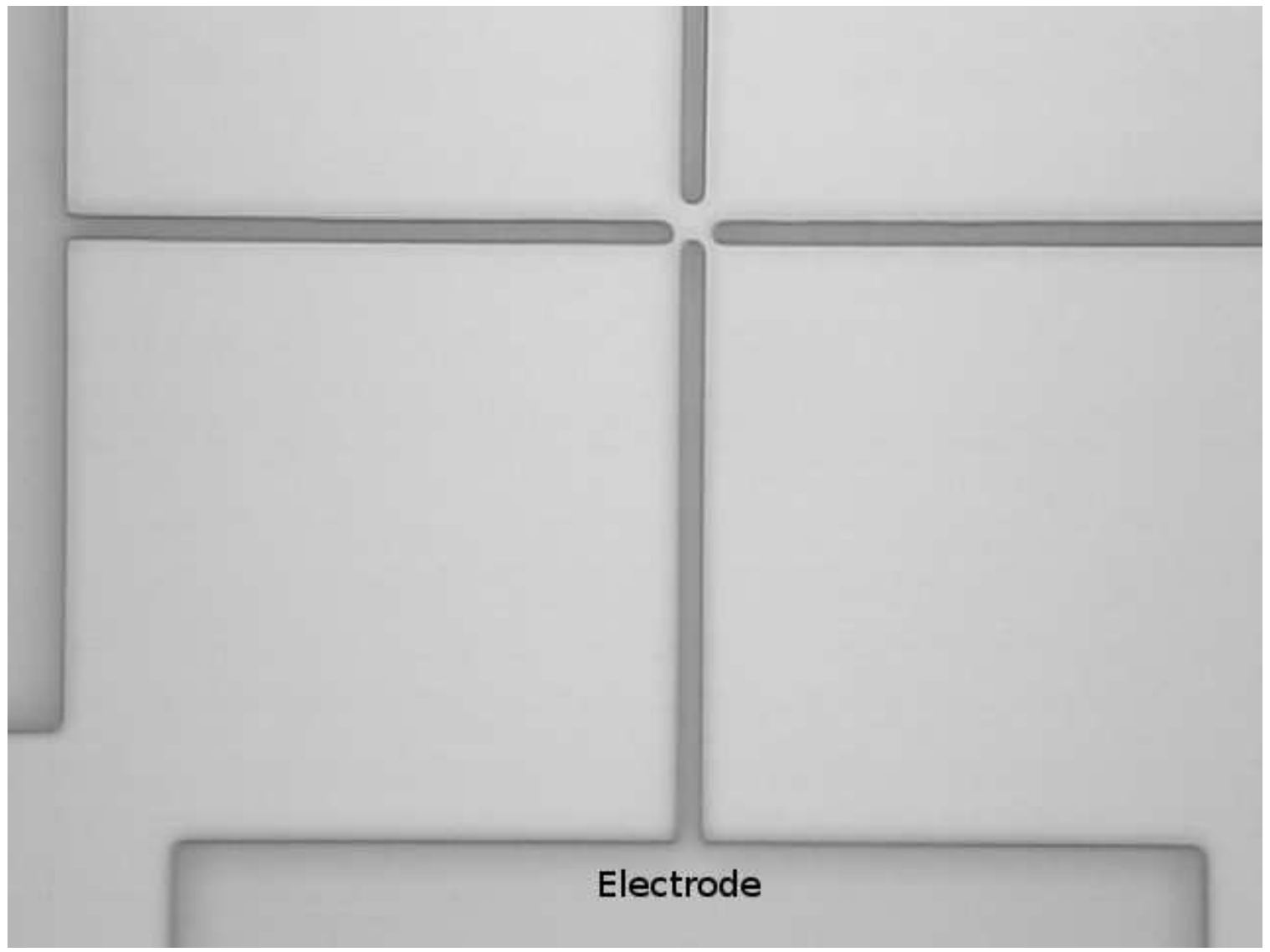

Figure 4.19: The initial DEP pattern was simple and designed for a two-step process to make a CNT cross. The width of the fingers is $3 \mu \mathrm{m}$.

\section{Microphotolithography Development}

The original pattern for the microelectrode fingers was a simple cross geometry, with long fingers connecting to large touchpads, shown in Figure 4.19. While necessary for the CNT cross and molecular electronics, it has a major drawback of having only one gap per experiment, leading to extremely low yield and a slow progress. Also, it was almost impossible to use this pattern as intended because the second pair of electrodes causes deposition direction to be altered by their presence. Dr. Craig Friedrich and Libao An had designed a pattern of microelectrode arrays which gave more data per experiment, and gave us permission to use it; this led to much more efficient experiments. The array pattern is shown in Figure 4.20.

From cutting substrates through patterning, film coating and liftoff, a single substrate patterned 


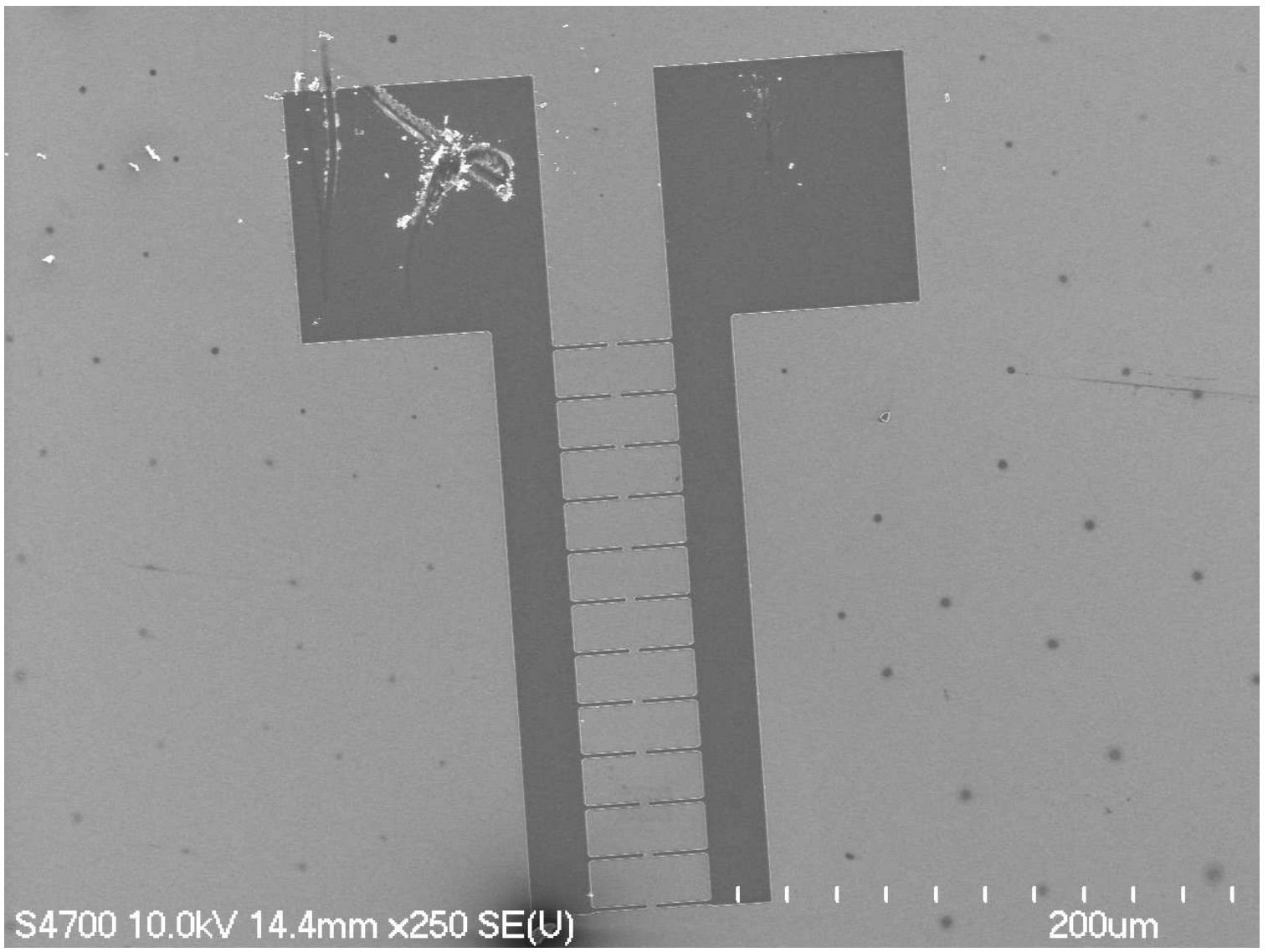

Figure 4.20: An SEM image of a microelectrode finger array pattern. The gap between the fingers is $2 \mu \mathrm{m}$. The large squares are touchpads used to connect the microelectrodes to an external circuit through the use of a probe station. The scratches are due to contact with microprobes. 
for dielectrophoresis is a significant time investment. During the course of investigating the microelectrode fingers, it was necessary to increase the yield of usable devices that came out of the processes. The major consideration is the cleanroom used to do the photolithography. First, it has no environmental controls, so humidity and temperature vary greatly from season to season, changing the exposure and development of photoresist. Also, the resists and UV lamp affect the necessary conditions as they age. Reoptimization led to a lot of time that could have been used for experiments being used just to find the new conditions. I made the decision to find a more consistent resist. After a great deal of experimentation and testing, we found the best course of action was to move away from the original, thicker resists (such as Shipley SC1827) to less viscous ones (like Futurrex PR-1000A). The downsides are that development of SC1827 was a longer process, allowing finer tuning while the less viscous resists have a much quicker development time, sometimes of only a few seconds. However, the less viscous resists are very even throughout the year, likely due to the higher levels of solvent in the resist.

Second, there is an inhomogeneity in the developed resists. Across a substrate patterned with the repeated microelectrode arrays, the gap pattern changes as you move across the substrate. This is a major problem, greatly reducing the number of usable patterns per patterned substrate because many of the patterns are shorted, with no gaps, shown in Figure 4.21.

The solution to this problem was to design a new mask with redundancies. Each DEP experiment contaminates a certain radius on the substrate, making it unusable for trials. Therefore, it results in no loss of usable space to make the microelectrode arrays bundled in small groups of differing gaps, shown in a schematically in Figure 4.22 .

\section{PECVD Experiments}

The microelectrode fingers are fabricated using photolithography techniques, which are described in more depth in Appendix A. The substrate is p-type silicon with a $120 \mathrm{~nm} \mathrm{SiO}_{2}$ layer, which are cleaned with acetone and then isopropyl alcohol (IPA) and then baked at $120{ }^{\circ} \mathrm{C}$ to remove any residual moisture. HMDS was spin coated at $4000 \mathrm{rpm}$ for $40 \mathrm{~s}$ in order to limit moisture from contaminating the surface and to improve film adhesion. Next, the positive photoresist PR-1000A (Futurrex) was applied and spun at $4000 \mathrm{rpm}$ for $60 \mathrm{~s}$, and then resulting photoresist (PR) film is baked at $120^{\circ} \mathrm{C}$ for 120 s. A mask aligner (EVG) was used in hard contact mode in order to expose 


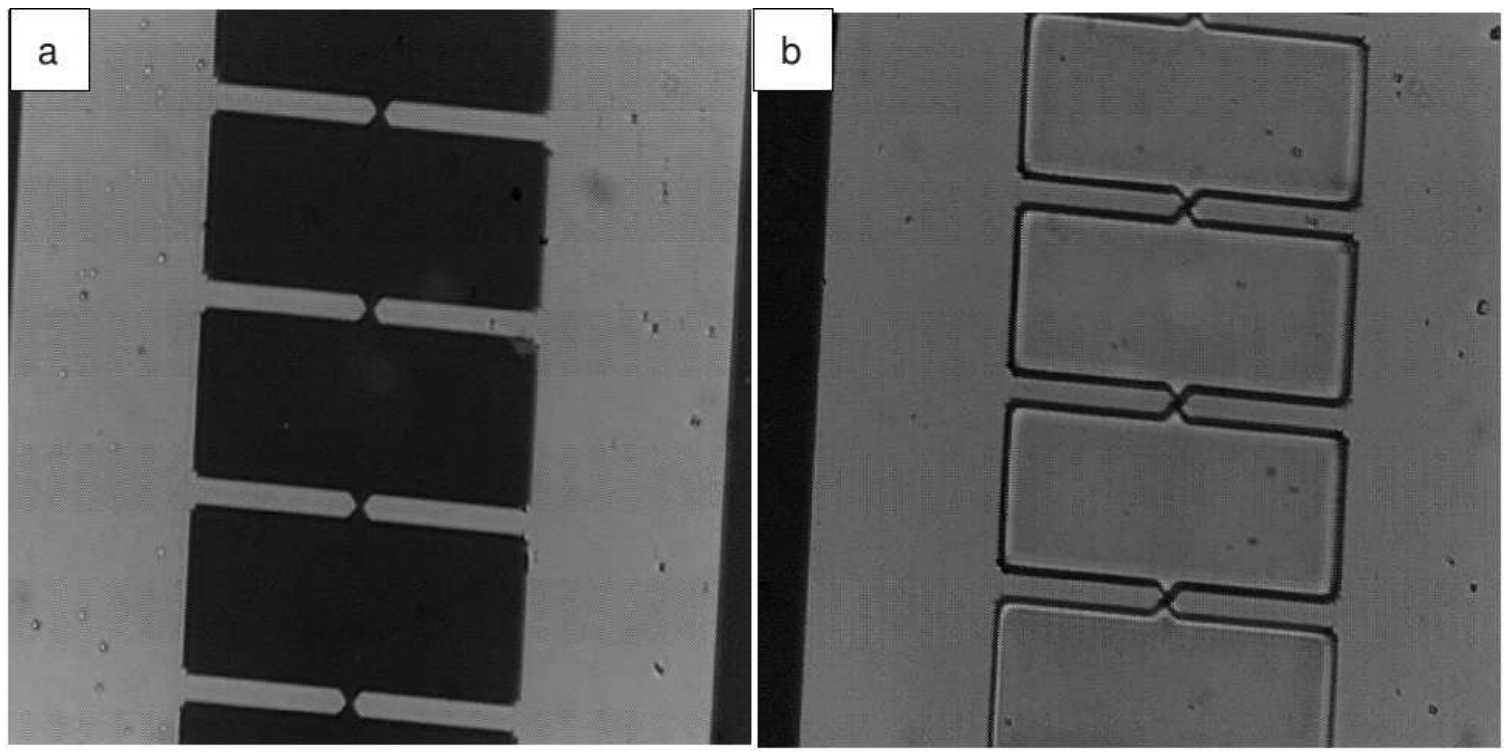

Figure 4.21: Over the surface of a substrate, the gap between fingers changes, making some unusable because of shorting. (a) and (b) are optical images of adjacent patterns; (a) has a proper gap and (b) is shorted.

the PR film for a time that varies by season (generally $7-12$ seconds). The substrates are developed in MF 319 developer for 10 - 60s (season dependent) before being rinsed in deionized water. Nickel was sputter coated to a thickness of $300 \mathrm{~nm}$ before lift-off. The lift-off step is accompanied by gentle mechanical scrubbing to finish the removal of excess metal, completing the fabrication. An example of a fully fabricated microelectrode finger array is shown in Figure 4.20. The arrays of patterns were inspected optically for flaws and a list of approved (unbroken, unshorted) patterns was compiled.

As always, initial MWCNT solutions were made using the stock PECVD grown tubes. The entire $3.85 \mathrm{~mm}^{2}$ growth area was dispersed into $50 \mathrm{~mL}$ of ethanol by immersing the growth substrate into the solution during sonication. The sonicating bath was continued for 2 hours to facilitate unbundling of the CNTs. Before dielectrophoresis, the solution was re-sonicated for 30 seconds before each deposition. A wave generator and a probe station completed the external circuit, starting the AC field before the introducing the solution. A $10 \mu \mathrm{L}$ biological syringe was used to place a roughly $5 \mu \mathrm{L}$ drop of the MWCNT-ethanol solution directly on the pattern and allowed to dry before the AC field was turned off.

With the PECVD tubes we tested to see if we saw the same frequency dependent phenomenon in 


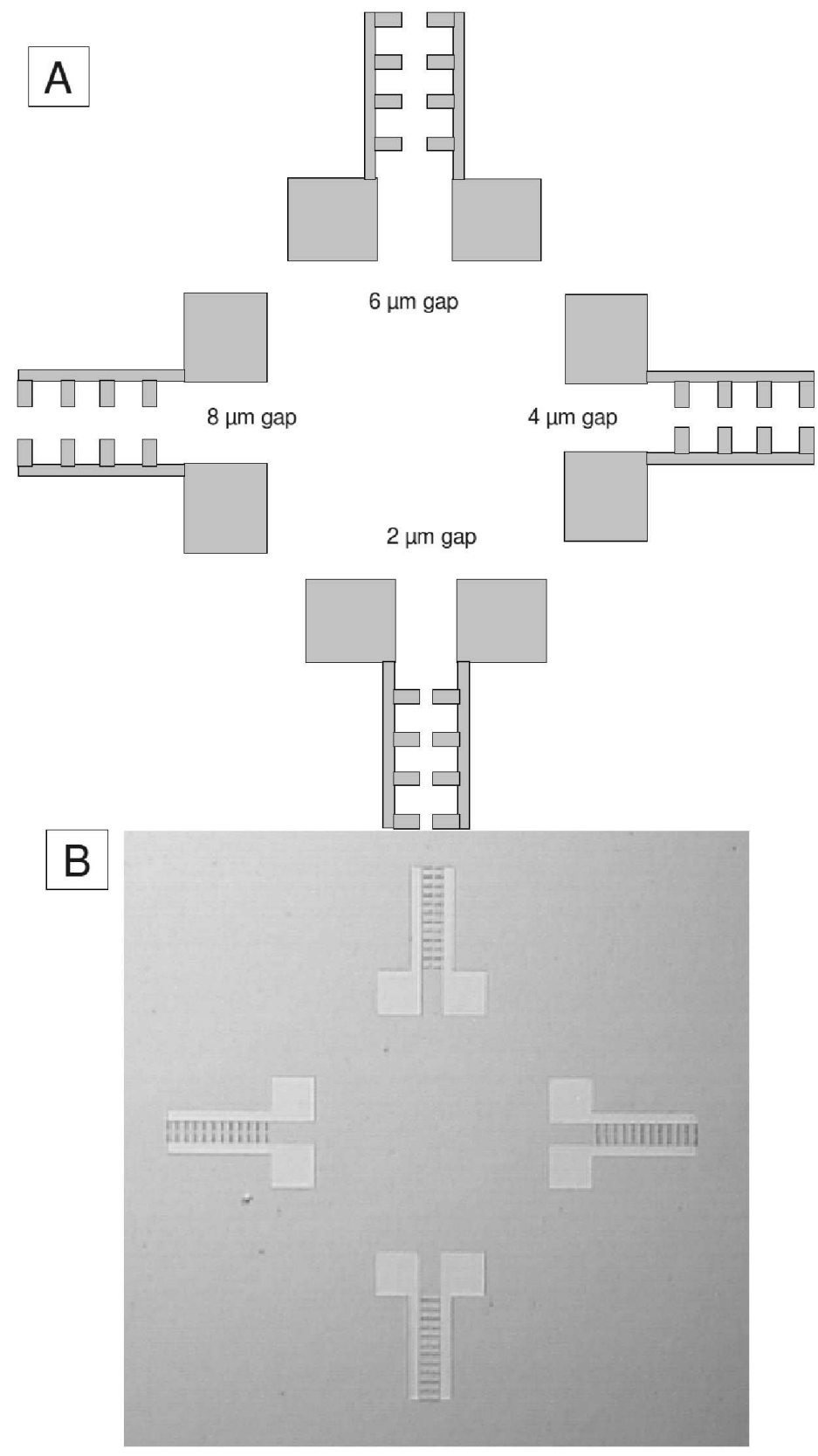

Figure 4.22: (A) A schematic of the improved microelectrode finger array pattern, designed to have a tolerance for the changing gap size as the pattern is repeated over a macroscopic substrate size. (B) The actual pattern deposited on a substrate. 


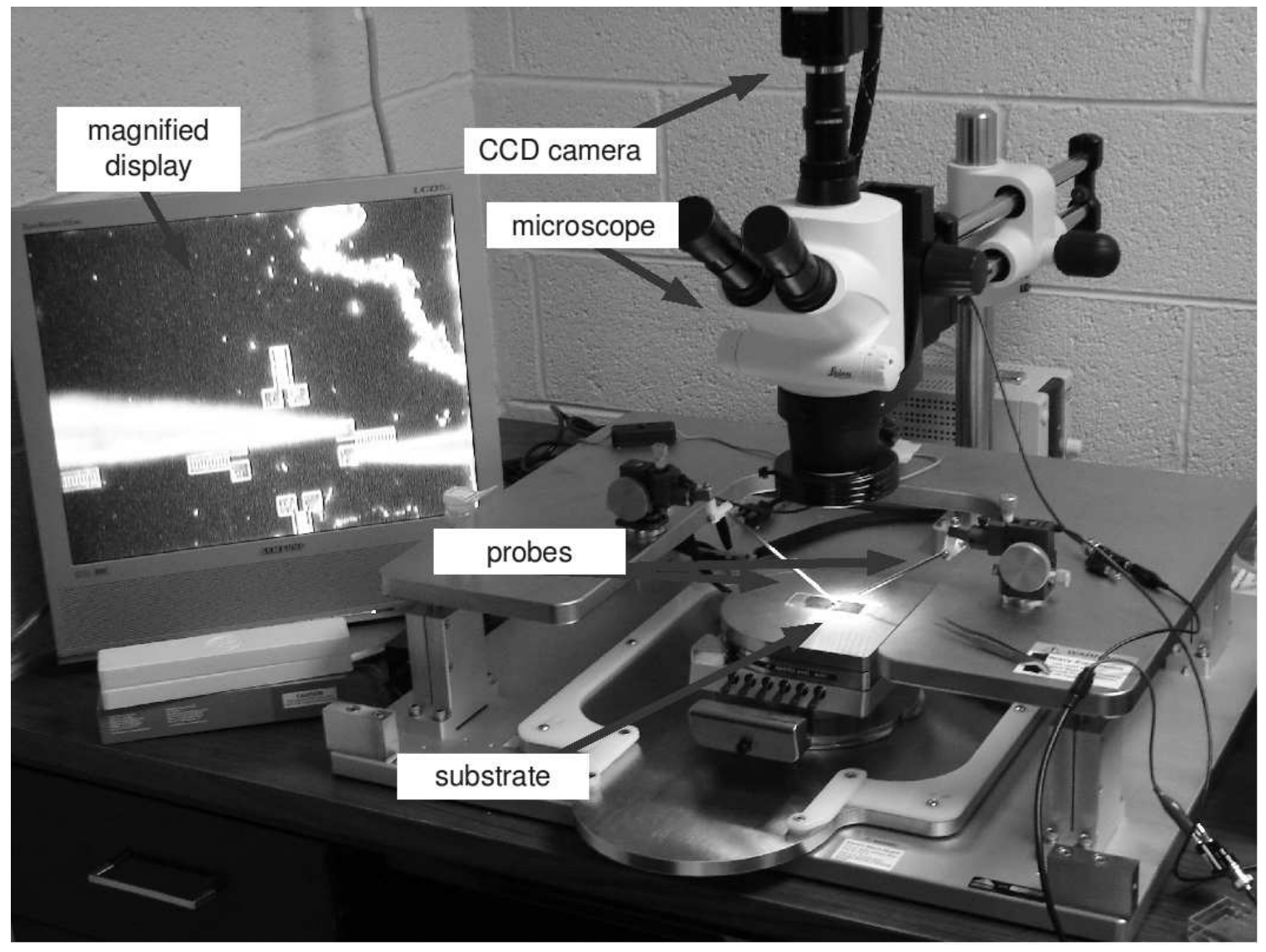

Figure 4.23: The experimental setup of the dielectrophoretic deposition. 


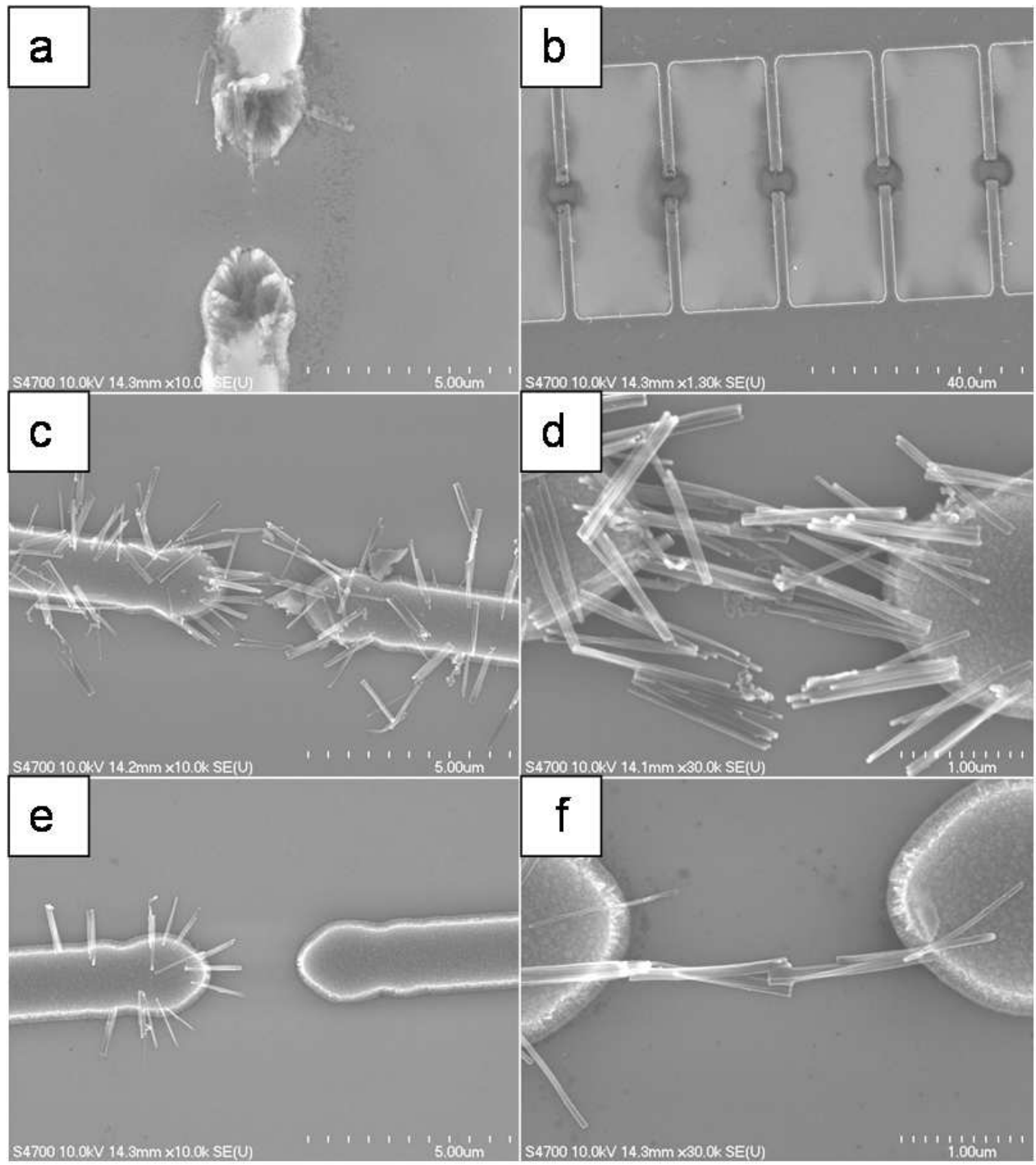

Figure 4.24: Effect of frequency for dielectrophoretic deposition of PECVD grown MWCNTs. Voltage was held at $30 \mathrm{~V}_{p p}$. (a)-(b) Deposition at $50 \mathrm{~Hz}$ shows little deposition and a very localized accumulation of impurities. (c)-(d) Deposition at $2 \mathrm{kHz}$ is extremely dense with no impurities in the region of interest. (e)-(f) Deposition at $2 \mathrm{MHz}$ is significantly less than at $2 \mathrm{kHz}$. 
the new microelectrode array geometry. Representative SEM of the different conditions are shown in Figure 4.24. All the previous observed trends are retained. Low frequencies still have poor deposition and contamination, Figure 4.24(a)-(b). In the kHz range, Figure 4.24(c)-(d), deposition is clean and very dense, but drops off considerably in the MHz range, Figure 4.24(e)-(f). So we know now that we have two routes of controlling the density of dielectrophoretically deposited MWCNTs, by voltage and by frequency in the range of $\mathrm{kHz}$ to $\mathrm{MHz}$. All that remains is to show this result with clear, clean connections made my thermal CVD MWCNTs, the goal all along.

\section{TCVD Experiments}

Having shown the result for DEP of PECVD tubes on microelectrode fingers and having achieved quality surfactant-free dispersions of TCVD MWCNTs by this point, it was time to combine the techniques. The experiments were carried out in the same manner with two exceptions. First, the CNT dispersions were made by $4-9 \mathrm{~mm}^{2}$ of TCVD growth area in $20 \mathrm{~mL}$ of 200 proof ethanol. Second, the electrodes were patterned with $20 \mathrm{~nm}$ Cr under $40 \mathrm{~nm} \mathrm{Au}$. The final result is shown in Figure 4.25.

Here we have unequivocally reached our goal of clean, untangled, surfactant-free dielectrophoretic deposition of thermal-CVD grown MWCNTs spanning the electrode gap with density tunable by both $\mathrm{AC}$ field strength and AC frequency. Figure 4.25(a) shows a maximum dispersion with a high $\left(32 \mathrm{~V}_{p p}\right)$ field strength and a $50 \mathrm{kHz}$ frequency. By either decreasing the field strength to 3.2 $\mathrm{V}_{p p}$, Figure $4.25(\mathrm{~b})$, or increasing the frequency to $2 \mathrm{MHz}$, Figure $4.25(\mathrm{c})$, the density of deposited

MWCNTs drops appreciably. Under these conditions, roughly $10 \%$ of the gaps do not have a connection, and preliminary numbers suggest that roughly $1 / 3$ of the gaps have single connections, and the remaining $2 / 3$ are few $(2-4$ tube) connections. By doing both at once, Figure $4.25(\mathrm{~d})$, deposition is almost completely suppressed.

\subsection{Summary}

Surfactant-assisted dispersion of MWCNTs was investigated under both horizontal and vertical configurations. Vertical dispersion resulted in tubes having a preference for aligning along the direction of fluid flow, and increasing the surfactant density reduced the curling of tubes. The 

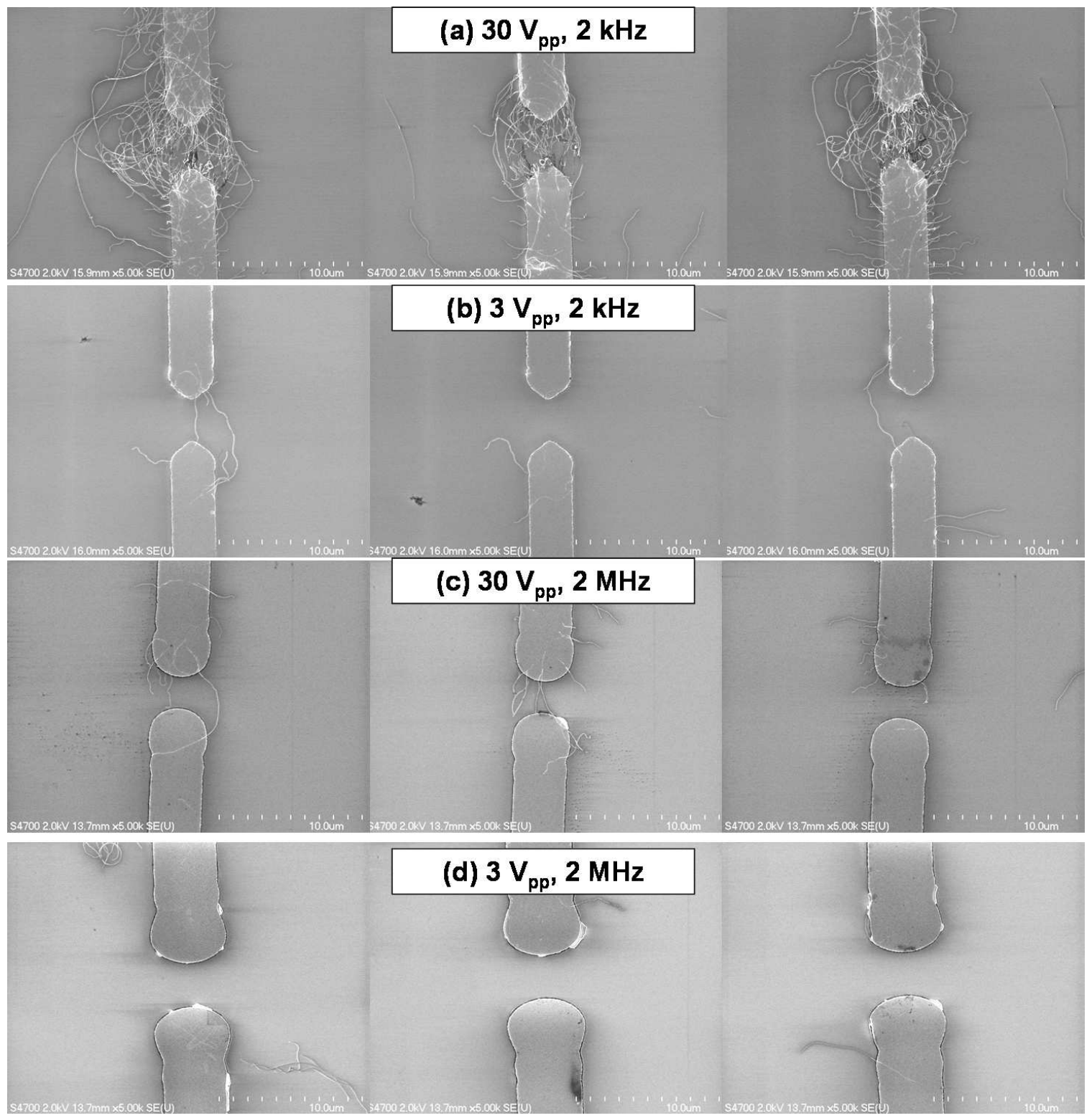

Figure 4.25: Representative SEM showing the tunability of DEP deposited MWCNT density by both AC field strength and AC frequency, and the types of connections found at each condition. (a) The maximum achievable deposition density for dispersions of this concentration. By (b) decreasing the field strength (c) or increasing the frequency we can tune the density to lower values. By doing both at once, CNT deposition is almost completely suppressed (d). 
vertical dispersion technique was used with photolithography to fabricate devices in which one or more MWCNTs connect electrodes.

Dielectrophoresis was investigated and compared to the current theory. Experiments shows that for MWCNTs deposited in flat electrode gaps (1) higher voltages mean more deposition, (2) low frequency $(\mathrm{Hz})$ has large amounts of contamination and few tubes, (3) medium frequencies $(\mathrm{kHz})$ show no contamination and very high tube deposition and (4) high $(\mathrm{MHz})$ frequencies are also clean, but have significantly fewer tubes deposited than the $\mathrm{kHz}$ range. We found that while theory can qualitatively explain these observations, because the experimental conditions invalidate many assumptions of the theory and there are many effects not accounted for, quantitatively they do not match. Because of the mismatch, it was unclear whether or not the results would be the same for microelectrode fingers. However, combining our surfactant-free dispersion of TCVD MWCNTs with our DEP experiments, we have shown we have clean, tunable MWCNT deposition that can result in single or few MWCNTs spanning the gap. 


\section{Chapter 5}

\section{Carbon Nanotube Modification and Bundling}

For many MWCNT based devices it is necessary to modify the CNTs chemically or physically, to tailoring the properties of the resulting device. Some of these methods were examined initially for the purposes of enhancing field emission and to investigate functionalized carbon nanotubes as background for making sensors and molecular electronic devices. This chapter describes the methods I have examined and some of the results and important observations that work has borne out. Acid etching of carbon nanotubes was a project that I started, then involved Benjamin Ulmen and is being carried on now by Archana Pandey.

\subsection{Acid Etching}

During the growth of the MWCNTs, the metal catalyst particle remains. For experiments dealing with field emission and electrochemistry, metal particles cause unwanted effects and are undesired. A simple acid bath is used to preferentially etch the catalyst particle, leaving the tubes intact. For the PECVD tubes, the catalyst particle is at the tip, so etching leaves tubes that are open at the top. Nickel is easily etched by nitric acid, so the process for etching the PECVD MWCNTs is as follows: (1) five minutes immersion in nitric acid, (2) rinsing in toluene to remove residual acid, (3) 


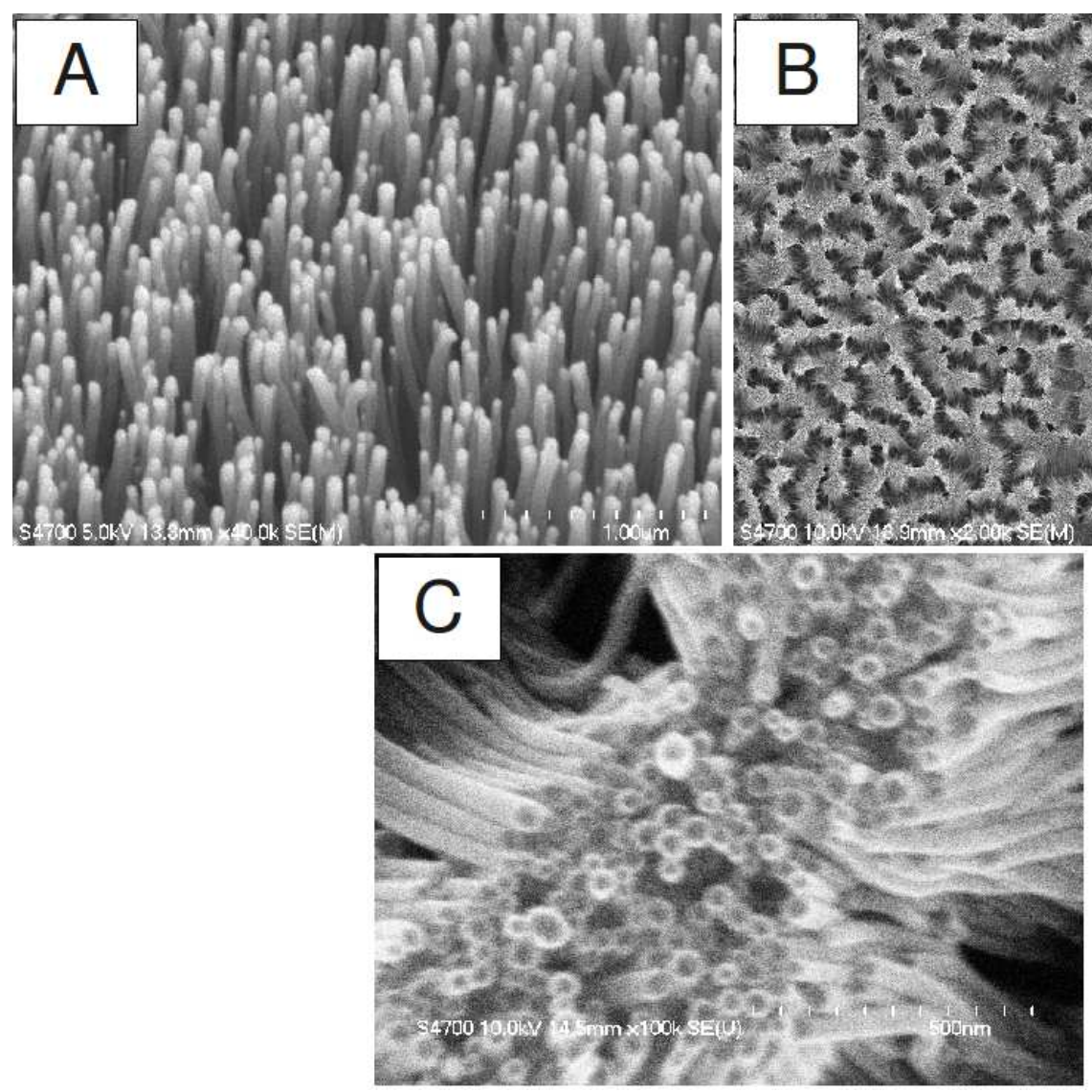

Figure 5.1: SEM images of PECVD grown MWCNTs (A) pre and (B)-(C) post catalyst removal by acid bath. (B) The tubes bundle into ridges all over the sample. (C) A closer view of the bundled tubes reveal the opened tips.

rinsing in deionized water to remove the toluene. SEM images before and after etching are shown in Figure 5.1.

While the particles are clearly removed, every sample exhibits a bundling of the tubes brought on by the immersion in liquid. Attempts to minimize the bundling by various insertion and extraction methods did nothing to prevent it. This is a very important side-effect and has impacted our field emission (as discussed below) and molecular electronic devices (in the chapter Molecular Electronics Devices I). 


\subsection{Enhancement of Field Emission by Etching}

Electron field emission is a process in which electrons are emitted from a sharp conductive tip due to a high electric field. The properties of semi-metallic carbon nanotubes, such as their high electrical and thermal conductivity, make them excellent candidates for commercial field emitters. In addition, their high aspect ratio enables a large electric field enhancement and yields emission at moderate electric fields. As en example of application, the size of the carbon nanotubes makes possible ultraflat display technologies by utilizing arrays of CNTs as electron guns for each subpixel in the display $[115,116]$. However, though prototype devices have been demonstrated, their commercial potential has not been fully realized, mainly due to problems with emission stability [117, 118].

The work on field emission was started by Vijaya Kayastha and Benjamin Ulmen, and is being continued by Archana Pandey. My contributions to the projects were (1) investigation of the etching process, (2) growing and etching MWCNTs for use in the field emission experiments and (3) training others in the etching process. The test system used was a "hanging" electrode design, maintaining a precise spacing between the bottom electrode (cathode) and hanging top electrode (anode) of 1000 $\pm 10 \mu \mathrm{m}$. A schematic of the cylinder and photo of the chamber is shown in Figure 5.2. The PECVD MWCNT films were grown on low resistivity silicon $(1 \Omega \mathrm{cm})$ and held in place and electronic contact with silver paste. The chamber is evacuated to $2.0 \times 10^{-7}$ mbar before and during experiments.

We investigated the effect of tip removal and bundling by comparing field emission of as-grown PECVD MWCNTs with etched and bundled MWCNTs. Importantly, the growth conditions for all samples were identical and the Raman spectra showed no statistically significant difference in the graphitic order of the MWCNTs before and after etching. More specifically, when using Raman to find the graphitic to disordered peak ratio, we always find a variance ( $\pm 10 \%$ of the mean) on a single sample from measured position to position. Any observed changes (more or less graphitic order) after etching fell well within this variance. We therefore concluded that etching process did not have a significant impact on the graphitic order of the MWCNT arrays.

After conducting the experiments, it was found that the threshold field, defined as the electric field necessary to emit electrons at the level of $1 \mu \mathrm{A} / \mathrm{cm}^{2}$, was not affected by the process (the threshold field varies from device to device and is in the range of $2.6-3.1 \mathrm{~V} / \mu \mathrm{m}$, but it remains consistent on each device before and after etching). However, the bundled tubes showed a higher 

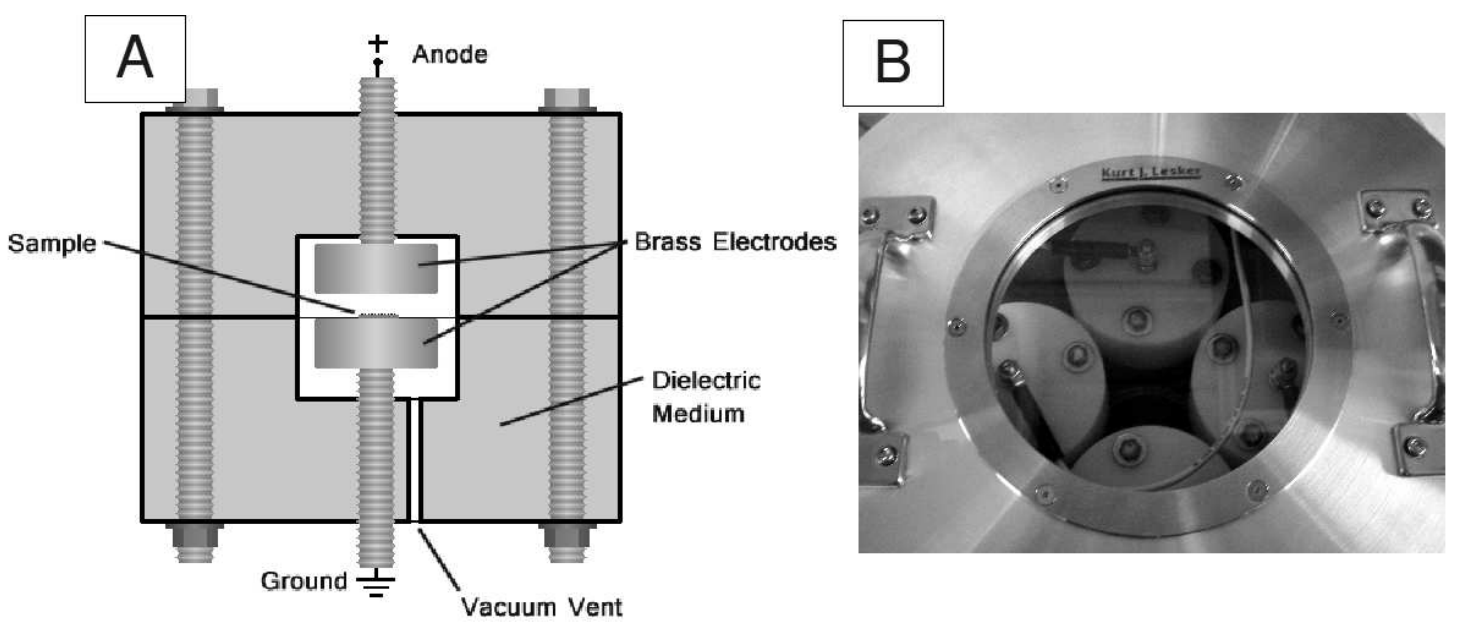

Figure 5.2: (A) Schematic of a field emission cylinder. (B) Photo of the field emission chamber through the top window, with several cylinders showing. Reprinted with permission from Reference [81].

long-term stability; the results of the study are show in Figure 5.3.

We believe the enhancement in stability comes from two major factors. First, residual metallic catalyst particles have a lower melting point than the $\mathrm{CNTs}\left(\sim 1452{ }^{\circ} \mathrm{C}\right.$ for bulk nickel versus $>3650$ ${ }^{\circ} \mathrm{C}$ for graphite) creates some unknown effects on the emission stability when significant Joule heating (heating induced by current flow) was introduced. The actual mechanism is still under investigation.

Second, the bundling plays a major role. Archana Pandey ran simulations using the $\mathrm{COMSOL}^{T M}$ MULTIPHYSICS software to investigate the bundling. Using parameters matching the PECVD MWCNTs used in the experiments and an applied field of $5 \mathrm{~V} / \mu \mathrm{m}$, she found the following. For an array of evenly spaced, vertically aligned MWCNTs, Figure 5.4(A), the highest field is on the edges (points 2 and $3, \sim 1.036 \times 10^{7} \mathrm{~V} / \mathrm{m}$ ) and not the center (point $1,6.713 \times 10^{6} \mathrm{~V} / \mathrm{m}$ ) because of the screening effect of neighboring tubes. When considering a bundle of tubes in a pyramid shape, Figure 5.4(B), the local field at the tip, point 2, is $5.9743 \times 10^{4} \mathrm{~V} / \mathrm{m}$, while the edges, points 1 and 3 , are $4.058 \times 10^{4} \mathrm{~V} / \mathrm{m}$ and $3.712 \times 10^{4} \mathrm{~V} / \mathrm{m}$. These values are two-orders of magnitude lower than the arrays of individual vertically-aligned MWCNTs. Lower local fields means a lower current density, which means less Joule heating and less thermally-induced stresses on the bundles. This lower, less stressed condition leads to the enhancement in long-term field stability. More complete results can be found in our publication, given as Reference [119]. 


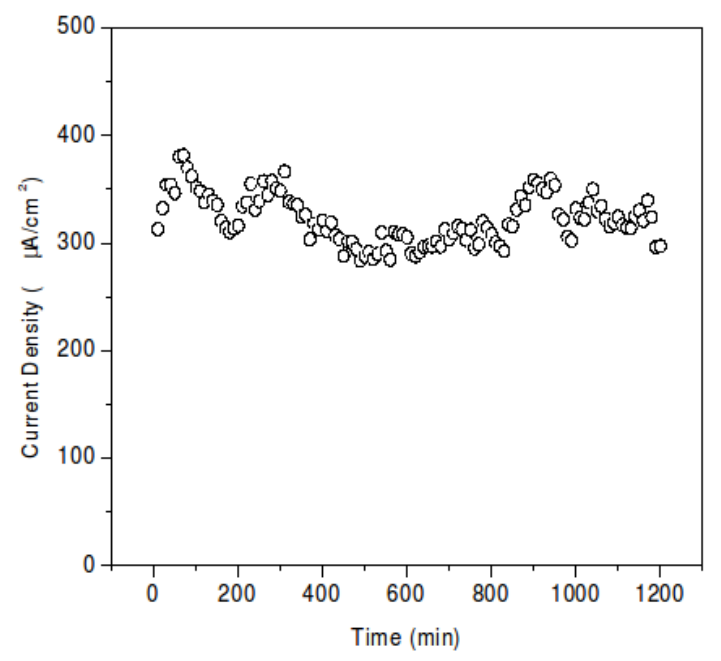

Stability graph for without etch $20 \mathrm{sccm}$

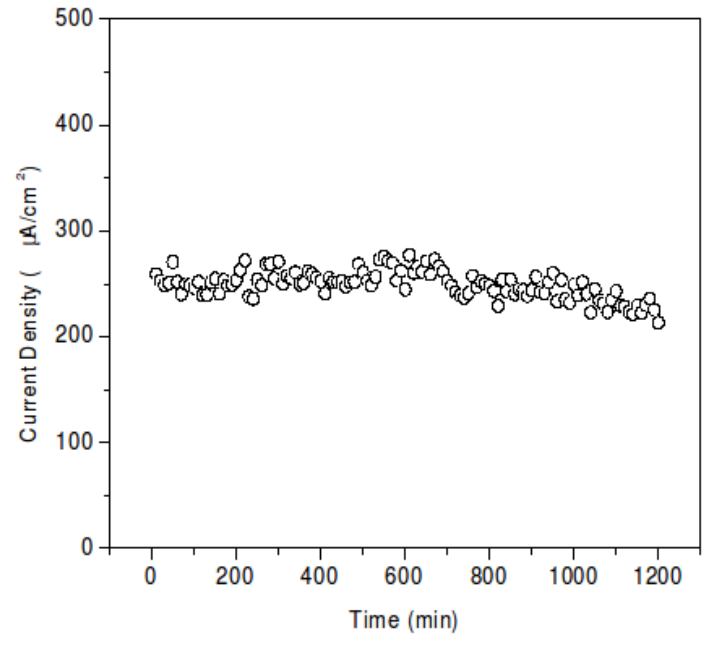

Stability graph for etch $20 \mathrm{sccm}$

Figure 5.3: A comparison of field emission stability for (A) as-grown PECVD MWCNTs and (B) etched and bundled PECVD MWCNTs. The etching and bundling process enhances the stability of emission.

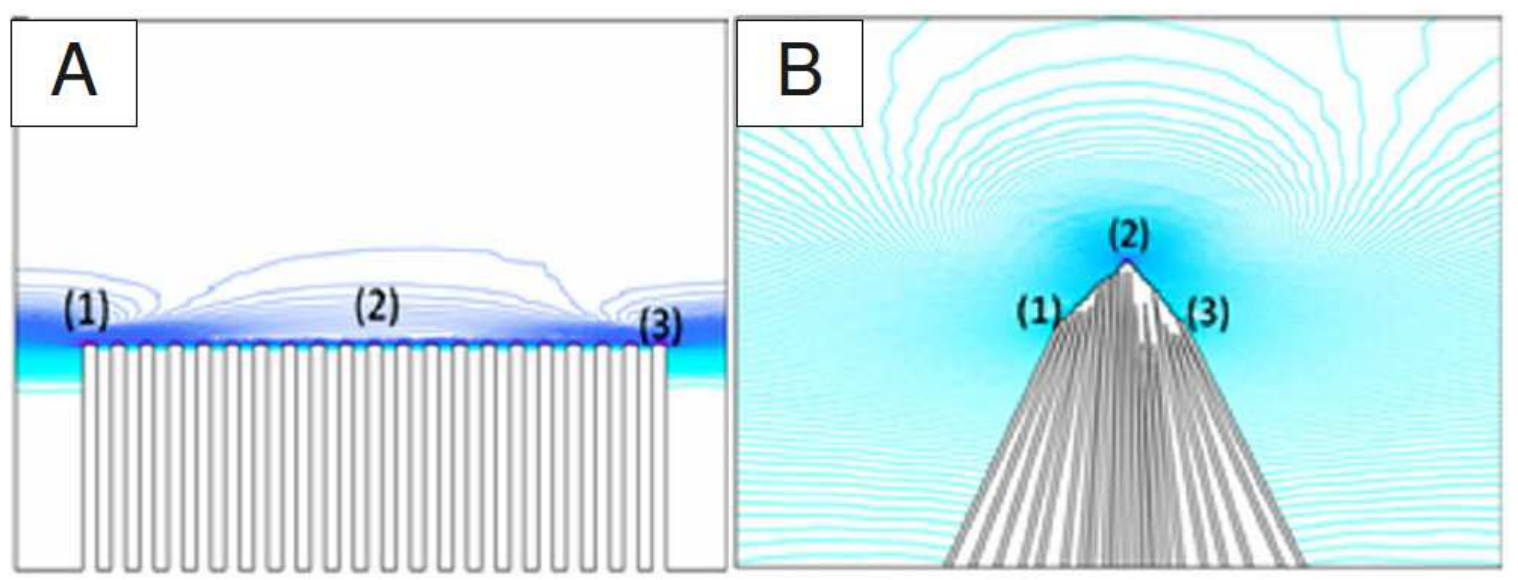

Figure 5.4: Simulated potential maps for (A) vertically-aligned MWCNT arrays and (B) conicallybundled MWCNTs. COMSOL simulations included with permission from Archana Pandey. 


\subsection{Carboxylation of Carbon Nanotubes}

Modifying the surface of carbon nanotubes is necessary for devices ranging from biological/chemical sensors to molecular electronic devices. Carboxylation, formation of a $-\mathrm{COOH}$ group, on the MWCNTs surface was the first investigation into functionalization because carboxyl groups are a very common bridge to countless functionalities. It was known that acid treatment removes amorphous carbon from the sidewalls and also can form the carboxyl groups on defect sites or any other place where there is an open bond, such as the opened tips [120,121]. We investigated this carboxylation process with our PECVD grown tubes.

Samples were immersed in a 3:1 sulfuric:nitric acid bath, for up to 48 hours. The carboxyl group should have a very strong signal in FTIR spectra, but none were observed. We believe that the layer of bundled tubes presented too small a cross-section to get an appreciable signal from the sample without a technique like attenuated total reflection, for which we lacked the necessary facilities. In order to (1) test that the carboxylation had taken place and (2) show that we had the capacity to use the - $\mathrm{COOH}$ functionality as a bridge to other modification, a fluorescent-labeled biomaterial was used, as detailed below. This work was done with the assistance of Dr. Dongyan Zhang, who carried out the labeling.

Bovine serum albumin (BSA, Sigma) was labeled with fluorescent isothiocyanate (FITC) using an EZ-LABEL ${ }^{T M}$ FITC Protein Labeling Kit (PIERCE). The FITC labeled BSA was then diluted with $25 \mathrm{mM}$ 2-[N-morpholino] ethanesulfonic acid (MES, Sigma) buffer to a concentration of 1 $\mathrm{mg} / \mathrm{mL}$.

The PECVD-grown MWCNT films were functionalized with carboxylic groups by immersion in a 3:1 mixture of sulfuric and nitric acids for $24 \mathrm{~h}$ at room temperature. $4.2 \mathrm{M}$ N-hydroxysuccinimide (NHS, Alfa Aesar) and 31.7 M 1-Ethyl-3-(3-diisopropylethyl-amine) carbodiimide hydrochloride (EDC, Alfa Aesar) were prepared separately by using a $25 \mathrm{mM}$ MES buffer. These NHS and EDC solutions were then used to activate the MWCNT film by dripping droplets of the NHS solution (total $100 \mu \mathrm{L}$ ) and EDC solution (total $200 \mu \mathrm{L}$ ) alternatively on the film. The MWCNT film was then incubated in a humidity chamber at $30{ }^{\circ} \mathrm{C}$ for 30 minutes to form stable active esters on the MWCNTs. The activated MWCNT film was then removed from the EDC/NHS mixture and added with $100 \mu \mathrm{L}$ FITC-labeled BSA and incubated overnight ( 24 hours) at room temperature in a 
darkroom. During the process, the active ester reacted with the amine groups of BSA to form amide bonds between the MWCNTs and the proteins. The incubated MWCNT film was then thoroughly washed and rinsed with deionized water to remove any un-bonded FITC-labeled BSA.

The labeling process was carried out for MWCNT samples that had been carboxylized by acid bath and a control group that had not. The signal of the FITC-label BSA covalently bonded on the MWCNTs was detected by photoluminescence using a Raman spectroscopy system with a HeCd excitation laser (wavelength $=325 \mathrm{~nm}$ ). The results are shown in Figure 5.5. The etched MWCNTs showed a fluorescent peak distinctly lacking in the unetched sample. We have shown that the carboxylation by acid bath is a success.

A number of caveats must be mentioned. First, even though we achieved functionalization, the signal is weak and undetectable by FTIR, even though the-COOH's IR signal is very strong. Second, we have no indication of the spatial distribution of functionalization sites on the MWCNTs; namely, whether they cover the sidewalls or are only found on the opened tips. However, we know from SEM analysis (such as Figure 5.1) most of the sidewalls are covered and all the tips are open. The carboxyl groups should preferentially modify the tips since they have the highest concentration of available dangling bonds. Therefore, the weak signal is probably mostly at the tips, and very few are on the sides. For many purposes, such as molecular junctions, this is not ideal.

\subsection{Glucose Sensors}

With the proof that we could carboxylize carbon nanotubes, we began to tackle devices. The first such device is a glucose sensor.

In 2007 the CDC estimates $7.8 \%$ of the US population had diabetes, and the percent is rising [122]. Such high numbers lead to a large demand for highly selective, sensitive glucose sensors. It has been reported that arrays of polymer-embedded MWCNTs can be modified to fabricate glucose sensors of this type that are selective when in solution with other chemicals present in vivo [28]. While Lin et al. focused on the selectivity of the device, we present further characterization, with initial results on detection limits and GOx site density.

This project is led by Archana Pandey, who carried out all the experiments. I serve as an advisor on this project; specifically, I helped Archana design the experimental setup, chose and ordered the 

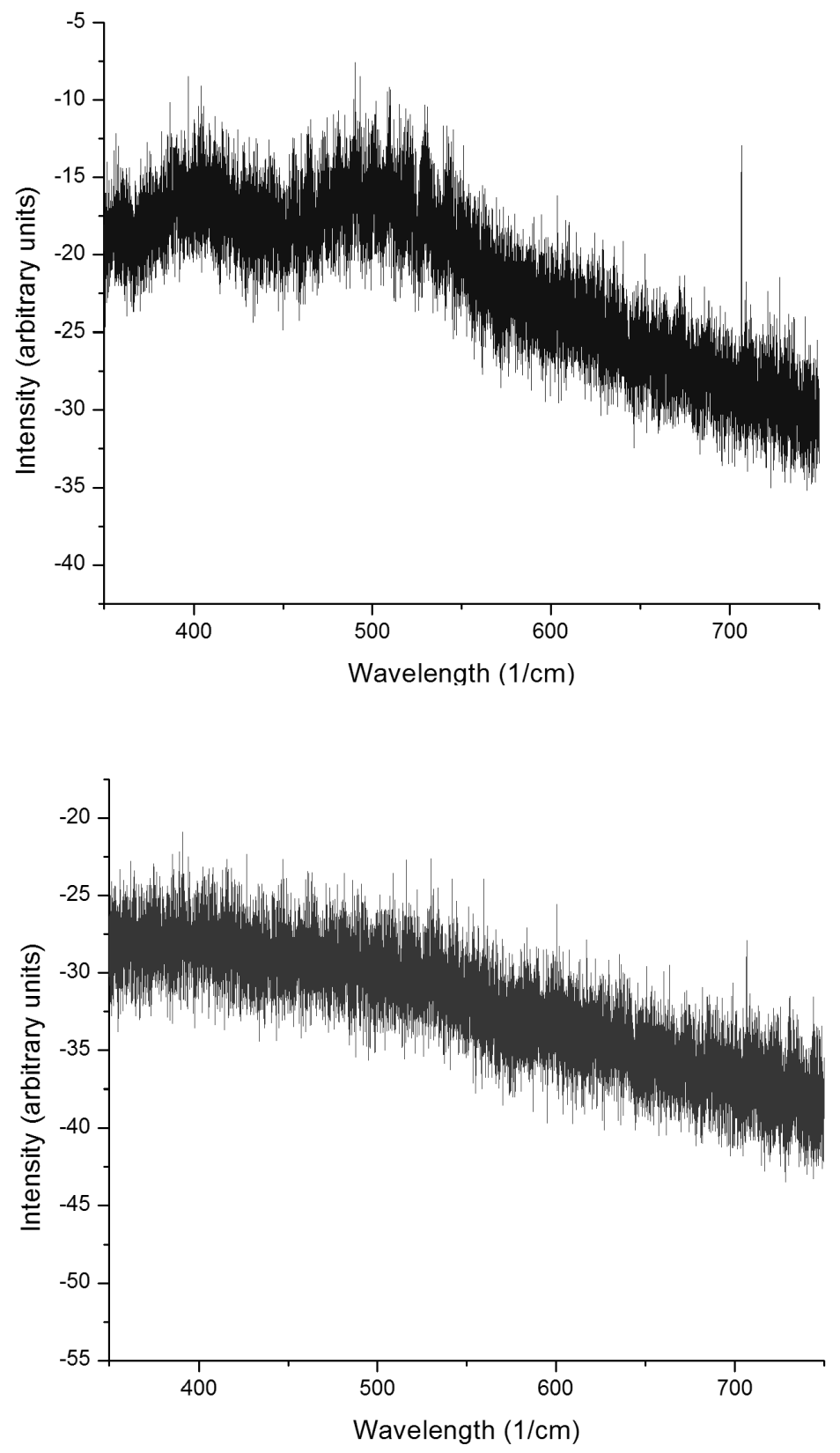

Figure 5.5: (Top)Representative PL spectra of FITC-labeled BSA functionalized MWCNTs containing a clear peak around $500 \mathrm{~cm}^{-1}$. (Bottom) Representative spectra of the control groups showing no peaks. 
materials necessary for the device and electrochemical cell, trained her on the electrochemistry and assist in compiling the data, running calculations and helping determine what further experiments needed to be done as the research progresses.

While preparing CNT based glucose biosensors, the common and most extensively used enzyme, glucose oxidase (GOx), is used to modify the CNTs [123, 124, 125, 126, 127, 128]. GOx is an enzyme discovered by Müller [129] as the catalyst responsible for the oxidation of glucose to gluconic acid in the presence of dissolved oxygen [130]. Since CNTs possess fast electro-catalytic speed and electron transfer rate, it is believed that CNTs can capture the electrons from the deeply embedded redox centers in GOx and act as transducers in CNT based glucose biosensors [131].

GOx is a dimer with two molecules of flavin adenine dinucleotide (FAD). The GOx enzyme binds to $\beta$-D-glucose and aids in breaking it into its metabolites. In the GOx catalyzed redox reaction, FAD works as the initial electron acceptor and is reduced to $\mathrm{FADH}_{2}$ - it is this accepting of electrons that we measure. Once $\mathrm{FADH}_{2}$ becomes oxidized by molecular oxygen $\left(\mathrm{O}_{2}\right)$, the oxygen is then reduced to hydrogen peroxide $\left(\mathrm{H}_{2} \mathrm{O}_{2}\right)$. The redox reaction catalyzed by $\mathrm{GOx}$ is:

$$
\text { Glucose }+\mathrm{O}_{2} \stackrel{\text { GOx }}{\longrightarrow} \text { Gluconolactone }+\mathrm{H}_{2} \mathrm{O}_{2}
$$

The first step in fabricating a sensor of this type is developing the array of embedded MWCNTs that serve as the nanoelectrodes for the sensor. As depicted in Figure 5.6, vertically aligned MWCNTs were grown directly on Ni-coated silicon substrates by our plasma-enhanced chemical vapor deposition technique, Figure 5.6(A). The entire substrate was then dip-coated in a mixture of poly(methyl methacrylate) (PMMA) and its developer (to reduce viscosity) to fully embed the MWCNTs, Figure 5.6(B). The film is then annealed at $100{ }^{\circ} \mathrm{C}$ to remove the solvents and harden the film. Finally, the surface was mechanically polished in order to remove excess PMMA and the MWCNT tips, exposing the opened MWCNTs, Figure 5.6(C).

Once the nanoelectrode arrays are completed, the exposed portion of the tubes needs to be carboxylized. The first generation of devices we made used the acid bath method described in Section 5.3 to form the carboxyl group and yielded working devices. This was later modified to electrochemical modification which is a faster process. The electrochemical carboxylation was done was making the nanoelectrode array the working electrode in an aqueous electrochemical cell, as 

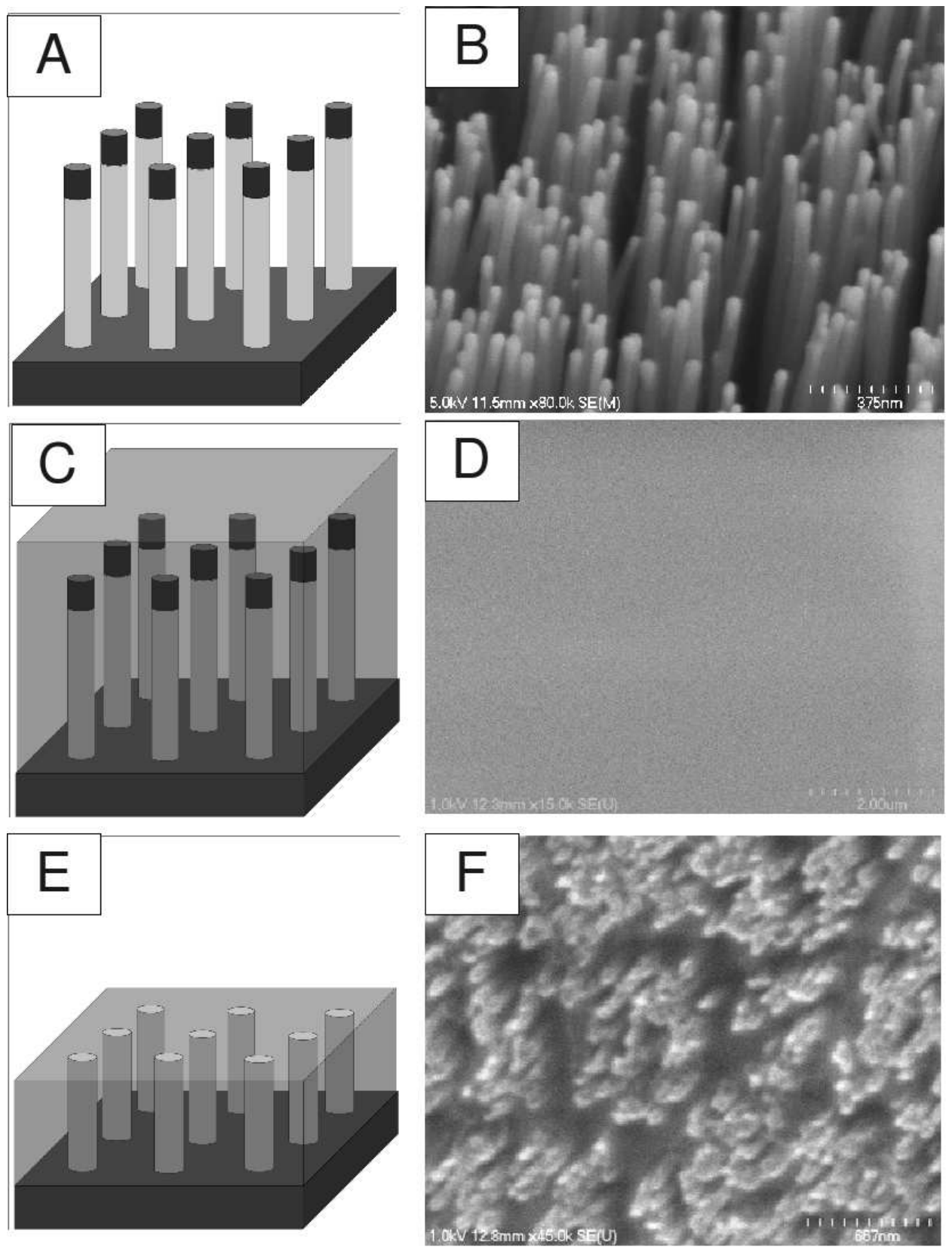

Figure 5.6: Diagrams and SEM of the nanoelectrode array fabrication process. (A)-(B) First, vertically aligned MWCNTs are grown by a plasma enhanced CVD method. (C)-(D) Next, the nanotubes are embedded by immersion in PMMA and baked to harden. (E)-(F) Finally, the surface is mechanically polished to remove the catalyst particles at the carbon nanotube tip, leaving opened tubes. SEM images included with permission of Archana Pandey. 

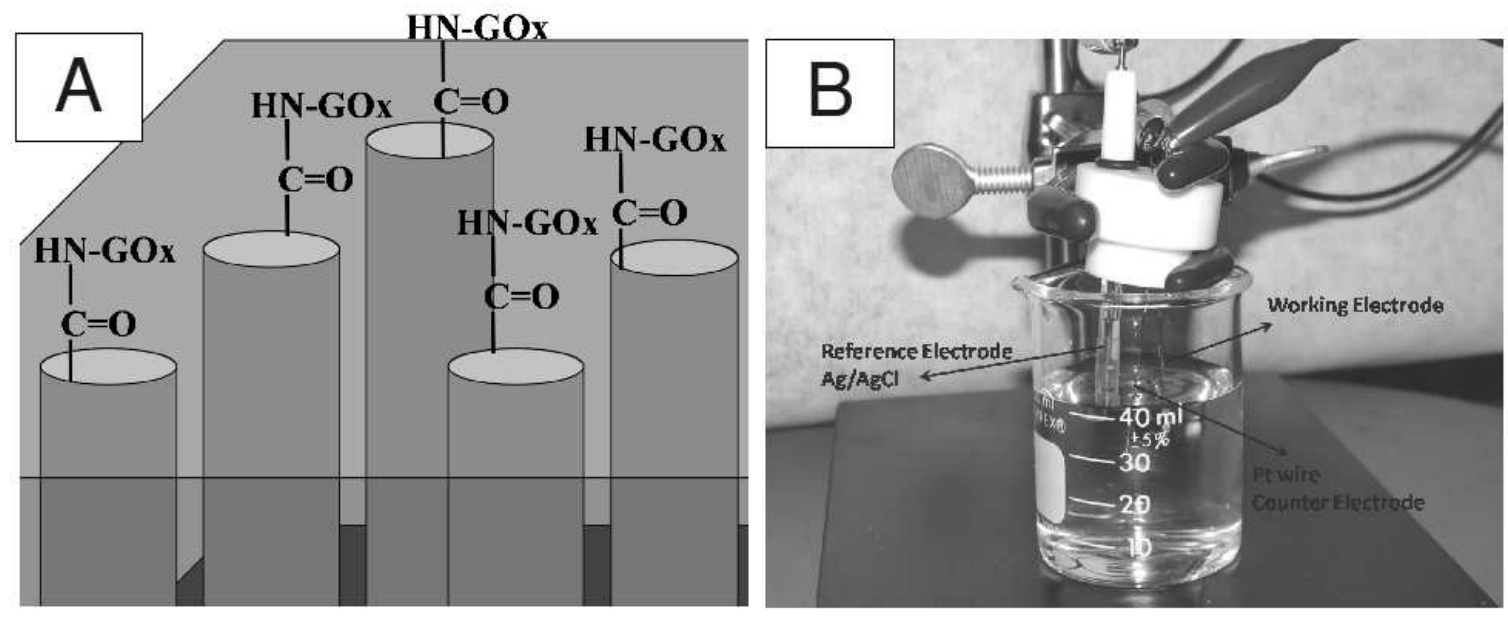

Figure 5.7: (A) Diagram illustrating the immobilization of GOx onto the open tips of the carbon nanotube nanoelectrode array. (B) The three electrode aqueous electrochemistry cell composed of the nonoelectrode array working electrode, platinum wire counter electrode and $\mathrm{Ag} / \mathrm{AgCl}$ reference electrode.

shown in Figure 5.7(B). $1 \mathrm{M} \mathrm{NaOH}$ was added to the cell and a $2.8 \mathrm{~V}$ was applied to the working electrode (vs. $\mathrm{Ag} / \mathrm{AgCl}$ ) for 2 minutes to complete the modification.

With the - $\mathrm{COOH}$ groups in place, the final chemistry took place as detailed by Lin et al. [28]. Briefly, the arrays were immersed in a solution of water, 1-ethyl-3-(3-dimethylaminopropyl)carbodiimide (EDC) $(10 \mathrm{mg} / \mathrm{mL})$ and N-hydroxysulfo-succinimide (sulfo-NHS) $(15 \mathrm{mg} / \mathrm{mL})$ allowing the reaction to take place for 3 hours at room temperature in order to form amide linkages between the amine residues and carboxylic acid groups. Afterward, the samples were rinsed thoroughly with deionized water. Finally, the arrays were immersed in $20 \mathrm{~mL}$ of GOx solution $(0.1 \mathrm{M})$ prepared in phosphate buffer solution ( $\mathrm{pH}$ 7.2). GOx immobilization was allowed to occur at room temperature for 3 hours. The immobilization is shown in the cartoon in Figure 5.7(A).

For testing, the glucose sensor is incorporated into a three-electrode electrochemical cell as the working electrode, employing a phosphate buffer solution $(20 \mathrm{~mL}, \mathrm{pH} 7.2)$ as the solvent, a platinum wire as the counter electrode and an $\mathrm{Ag} / \mathrm{AgCl}$ reference electrode. The amperometric response was obtained using a computer controlled potentiostat (CHI 600D); all cells were allowed at least 500 seconds to equilibrate before adding the glucose to the system and observing current changes induced by glucose - GOx binding. 


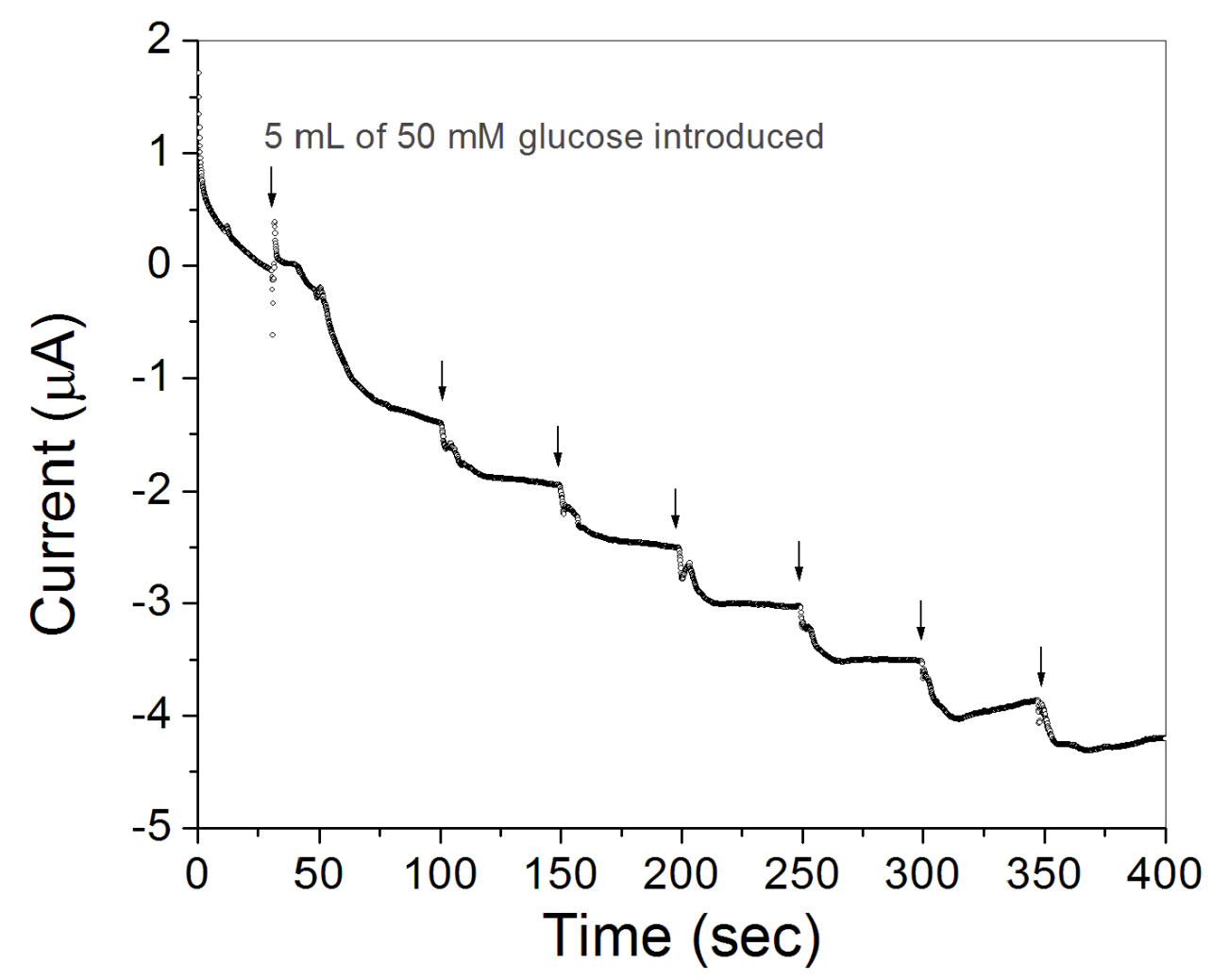

Figure 5.8: Amperometric response of the glucose sensor to successive additions of glucose. Each arrow indicates the addition of $5 \mathrm{~mL}$ of $50 \mathrm{mM}$ glucose solution to the $20 \mathrm{~mL}$ cell and induces increasing negative current.

The system's response to successive additions of $5 \mathrm{~mL}$ of $50 \mathrm{mM}$ solution is shown in Figure 5.8. It is clear that for each addition of glucose, the current becomes increasingly negative as the GOx-modified electrodes accept electrons from the glucose. For the range of concentrations considered in this report, the sensor response is linear in nature, as shown in Figure 5.9. This allows a linear regression to calculate the detection limit. For three standard deviations above the blank, we estimate the detection limit to be $14.4 \mu \mathrm{M}$. This gives the glucose sensor a sensitivity of $410 \mathrm{nA} / \mathrm{mM} / \mathrm{cm}^{2}$, which is competitive with most other glucose sensors being developed [132]. Importantly, this calculation uses the growth area and not the exposed area of MWCNTs. This means that the sensitivity could be increased even further by increasing the MWCNT density. 


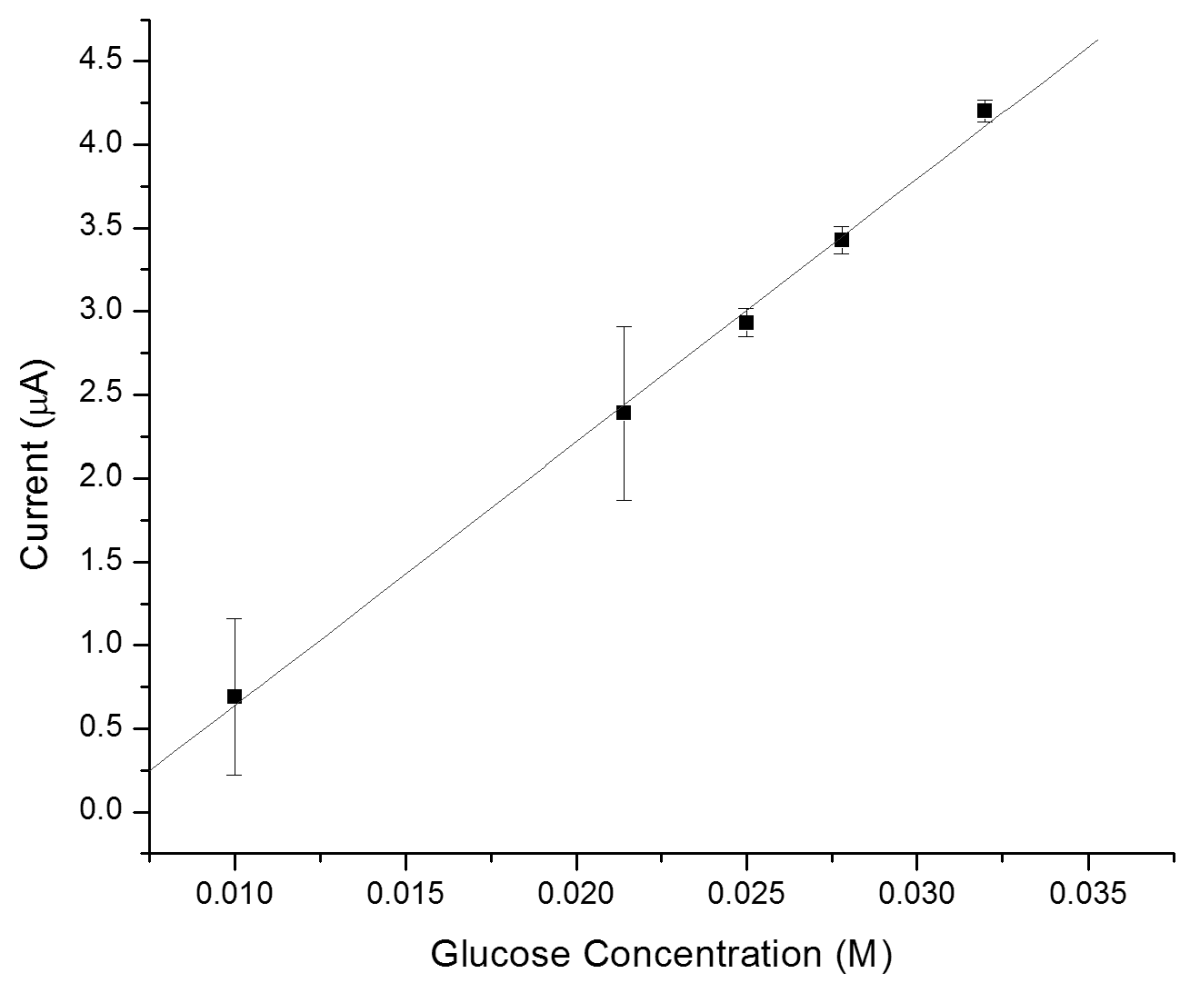

Figure 5.9: Sensor current response for different glucose concentrations. The points are derived from the data in Figure 5.8 with the value as the mean value of each plateau and the error bars represent the average deviation from the mean for each plateau. 
Figure 5.10 shows the response of glucose sensors to differing amounts of glucose. For the 0.44 $\mathrm{mM}$ and $0.87 \mathrm{mM}$ solutions, Figures 5.10(A) and (B), the response is short and the current returns to its initial value. When the concentration is a much higher $20 \mathrm{mM}$, the current increase is very pronounced, but never decays to its initial value within the $1500 \mathrm{~s}$ measurement. Initial order of magnitude calculations indicate that the average rate of glucose consumption for the cell is on the order of $1 \times 10^{-4} \mathrm{mmol} / \mathrm{s}$. Since the longest experiments for our $20 \mathrm{mM}$ solutions were $3100 \mathrm{~s}$ (not shown) and did not fully consume the glucose, we can estimate the device contains at least 0.31 mmol of GOx able to react with glucose. This corresponds to a lower bound of GOx site density of roughly $0.81 \mathrm{mmol} / \mathrm{cm}^{2}$. This initial calculation for the bound of the consumption rate is for the $20 \mathrm{~mL}$ solution size only; we have not yet investigated the effect of different solution volume for the same number of moles of glucose.

For more information on the glucose devices, including new analysis of area's affect on sensitivity, the cyclic voltammetry involved and the devices storage lifetime over a period of 8 months, see our upcoming publication or Archana Pandey's upcoming dissertation.

\subsection{Summary}

PECVD-grown MWCNTs were etched by immersion in an acid bath. This process was shown to remove the catalyst particle, but also to cause the tubes to bundle together in pyramid-like hills. The etching and bundling process was shown to improve the field emission stability of carbon nanotubes because of the removal of the easy-to-melt catalyst particle and the reduced local field the bundle is subjected to.

Acid etching was also used to carboxylize the MWCNTs. The carboxyl groups were not detectable by our FTIR, but were shown to exist by labeling them with a fluorescent molecule. Having proved we could carboxylize the MWCNTs, we used the method to make a first generation of glucose sensors. The functionalization method was replaced by an electrochemical method, and the sensors were shown to have a good response, with sensitivities comparable to existing devices, but with the potential to increase them further. 


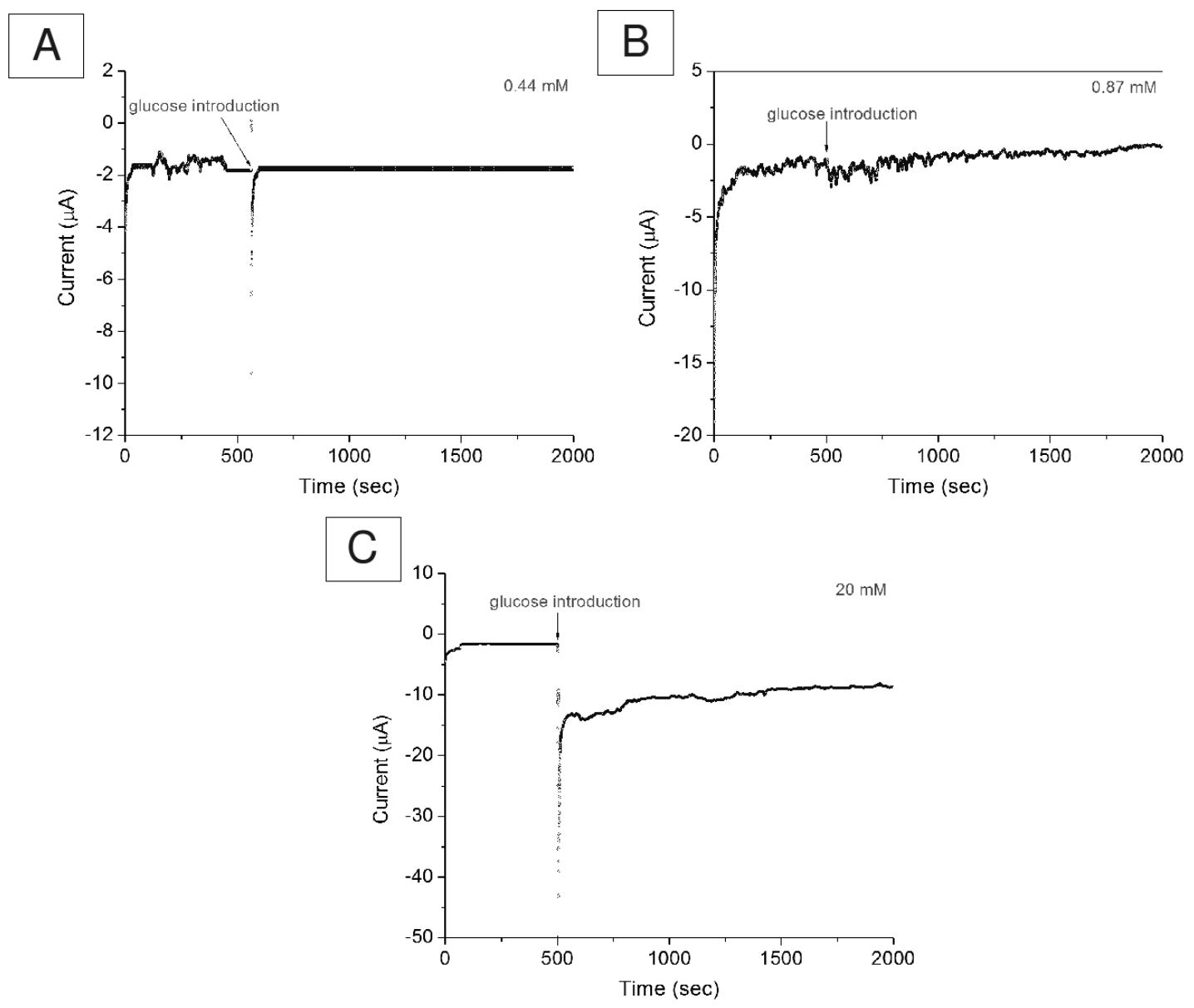

Figure 5.10: Amperometric response and recovery of glucose sensors after the addition of glucose in the amounts of (A) $0.44 \mathrm{mM}$, (B) $0.87 \mathrm{mM}$ and (C) $20 \mathrm{mM}$. With addition of glucose, the current increases in the negative direction, then decays to its original value over time as the glucose reacts and is used up by the GOx. 


\section{Chapter 6}

\section{Molecular Electronics Devices I}

\subsection{Background}

The term molecular electronics, sometimes called molectronics, can mean one of two things:

1. electronics taking advantage of the special properties of molecules, such as liquid crystal displays

2. electronic components constructed on the molecular scale with devices containing one or a few molecules

Some people handle this distinction by calling things falling into the first category molecular materials for electronics and the second as molecular scale electronics [133]. The devices under investigation here will not initially be molecular scale electronics, but will be able to be scaled down to such dimensions.

The origin of molecular scale electronics began with Robert Mulliken. Mulliken worked with Friedrich Hund to formulate the outline of a theoretical method for solving the Schrödinger equation for molecules, which Mulliken in 1932 named molecular orbital (MO) theory [134]. Following this, Lennard-Jones introduced the linear combination of atomic orbitals (LCAO) approach to develop molecular orbitals, which was extended by Mullikin into the self-consistent field (SCF) method which could calculate electronic states of small molecules [134]. This Nobel prize winning work serves as a basis for all later advances in theoretical calculation of molecular properties [134]. 
Mulliken also studied molecular donor-acceptor, charge-transfer complexes. In particular, he investigated internal charge-transfer in excited states of small molecules [134]. The molecules became ionic by electron transfer from one center to another. Mulliken explained that the ground state was a "supramolecular" orbital $\Psi_{0}$ consisting of a linear combination of an orbital $\phi_{D}$ (considered an electron donor, D) and an orbital $\phi_{A}$ (considered as acceptor, A), such that:

$$
\Psi_{0}=\alpha \phi_{D}+\left(1-\alpha^{2}\right)^{1 / 2} \phi_{A}
$$

where $\alpha$ is the atomic orbital (orthonormal) amplitude. The complex is stabilized by charge-transfer, and the excited state is:

$$
\Psi_{1}=\left(1-\alpha^{2}\right)^{1 / 2} \phi_{D}-\alpha \phi_{A}
$$

Modern modeling of molecular junctions, a molecule between two electrodes, use Mulliken's principles [134]. While molecular electronics was under study in the intervening years, it was the development of the scanning tunneling microscope in 1981 that led to a massive increase in investigation due to the ability to "see" the molecules for the first time.

Some of the more recent breakthroughs in the field include [134]: Reed and Tour demonstrated a molecular wire with the break-junction technique [135] and negative differential resistance [136], Metzger gave experiment proof of a working molecular rectifier [137], Joachim et al. demonstrated single-molecule transistors [138], Dekker et al. carbon nanotube transistors [139], Park et al. "single atom" single-electron transistors [140], several groups showed nanotube logic gates [141, 142, 143], and Heath and Stoddart et al. at HP fabricated 64-bit molecular RAM [144]. Work continues moving forward today, with new avenues opening all the time, and old ideas requiring study and refinement.

\subsection{Motivation and Initial Design}

As discussed in the introductory chapter of this dissertation, the silicon-based transistor is reaching its fundamental limits, and further increases in component density will require a change in approach. Further, as we move from traditional device architectures to new ones, we learn that we can make 
Table 6.1: Comparison of different novel RAM technologies.

\begin{tabular}{|l|l|l|l|l|}
\hline & Magnetoresistive & Ferroelectric & Phase & Molecular \\
& RAM $[145,146,147]$ & RAM $[148,149]$ & RAM[150, 151] & RAM $[9]$ \\
\hline Availability & Commercial & Commercial & Commercial & Research \\
Capacity & $4 \mathrm{Mbits}$ & $128 \mathrm{Mbits}$ & $1 \mathrm{Gbit}$ & $10^{11} \mathrm{bits} / \mathrm{cm}^{2}$ \\
Bit Size & $180 \mathrm{~nm}$ & $252 \mathrm{~nm}$ & $220 \times 400 \mathrm{~nm}$ & $30 \mathrm{~nm}$ \\
Read/Write Time & $35 \mathrm{~ns}$ & A few ns & $\sim 100 \mathrm{~ns}$ & tenths of seconds \\
Maximum Cycles & Unlimited & $>10^{12}$ & $>10^{8}$ & $<10$ \\
Main Attributes & Low Power, & Fast, Low Power, & Stackable bits, & High Density, \\
& Doesn't Degrade & Non-volatile & Non-volatile & Low Power, \\
& & & & Volatile \\
\hline
\end{tabular}

devices that have characteristics optimized for different applications. The simplest device made using transistors is memory, composed of bits, a component that can be put into one of two readable states. Some of the characteristics of novel types of memory available and under study are summarized in Table 6.1, and are discussed below.

Magnetoresistive and ferroelectric RAM are non-volatile memory, representing new technology that would be highly desirable for applications in which stability is the largest concern. Currently, magnetoresistance has a higher density, but slower read/write time than ferroelectric memory. Phase RAM is an example of memory that is very different from traditional technologies, where the bits are stored as glass elements change phase from amorphous to crystalline and back [150, 151]. In addition, phase RAM has been shown to move into three dimensional architectures by stacking device layers directly atop one another in order to increase bit density [151]. Molecular electronics is more of a broad term than the others, but the work cited in Table 6.1 represents some of the pinnacle work thusfar and was done by Heath et al.. Their memory array was composed of silicon nanowirerotaxane-titanium nanowire junctions and show clearly the pros and cons of the molecular approach - the potential for small required voltage and operational power, and extremely high component densities, but with the drawbacks of volatility, slow switching times and extremely low switching lifetimes [9].

A large amount of work is still required to understand the failure mechanisms and extend switching lifetimes of molecular junctions. It is the problem of low switching lifetime that we plan to investigate. For this reason, we decided to take up the approach of Richard McCreery et al. at Ohio State with carbon films and organic switches. McCreery's approach was to construct molecular devices utilizing strong bonds, such as the carbon-carbon bond, in the hopes that it would create more 


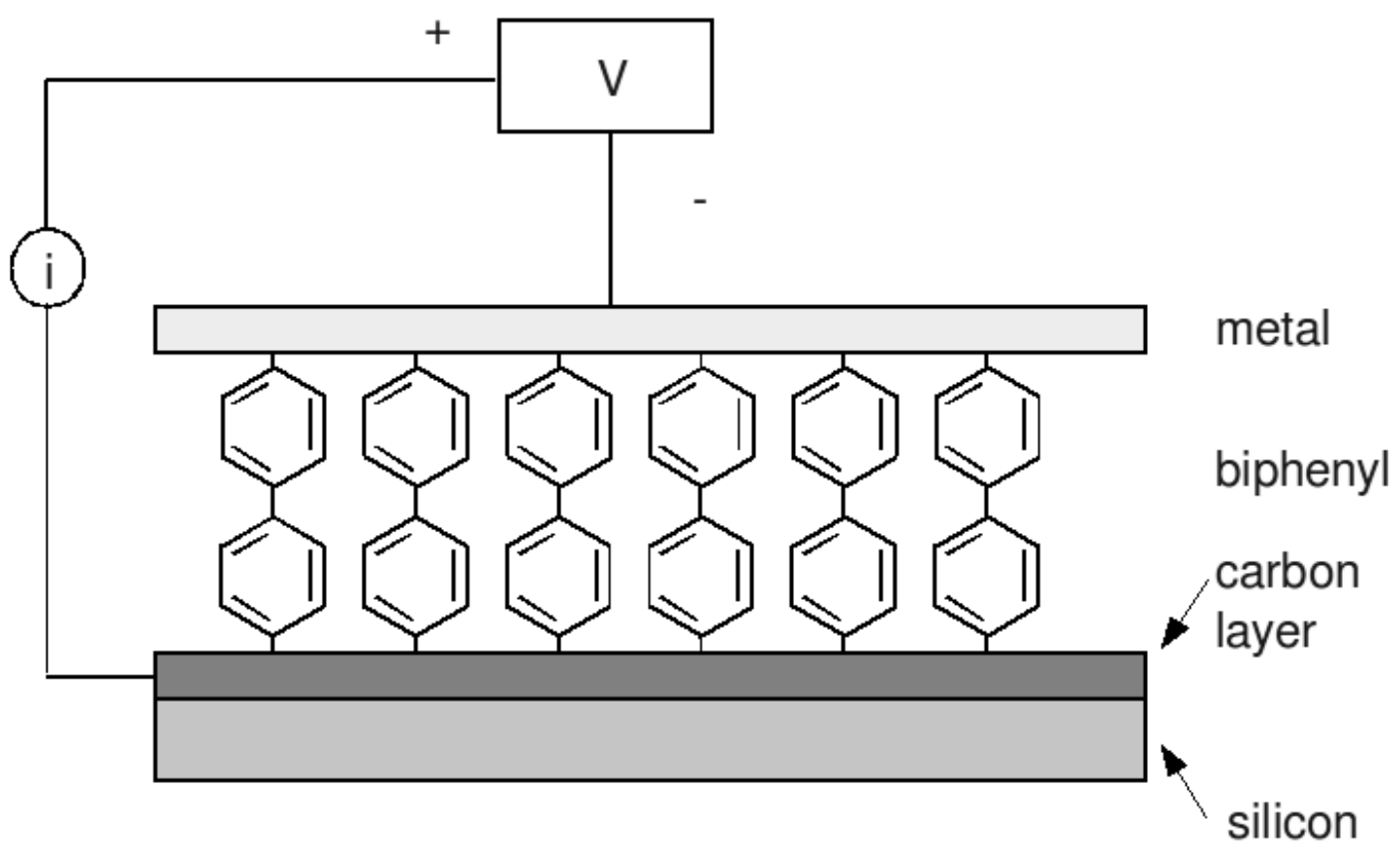

Figure 6.1: Schematic representation of the kinds of molecular junctions studied by McCreery et al.. A carbon layer is deposited on a substrate which is then functionalized by electrochemistry forming an organic monolayer on the surface, in this case biphenyl. A top electrode is formed by mercury drop or evaporation and is connected to an outside circuit for testing.

robust devices [152]. An example of a junction studied by McCreery is shown in Figure 6.1. His work focused on carbon films functionalized by non-aqueous electrochemistry. In order to complete the junction, a top contact was made using either STM, mercury drop or evaporated titanium. The carbon layer and top contact are connected to an external circuit in order to test the current-voltage response. McCreery found a variety of organic molecules exhibit switching behaviour between high and low conductance states upon sufficient applied voltages, two examples of which are shown in Figure 6.2.

Out of the molecules he investigated, we chose biphenyl $\left(\mathrm{C}_{12} \mathrm{H}_{10}\right)$ to act as our molecular switch because of its very clean switching behaviour, as shown in Figure 6.2(bottom). We would take his design a step further to create junctions composed of CNT-biphenyl-CNT formed by functionalized carbon nanotube crosses. If the test junctions worked, the geometry to be extended to arrays of junctions, forming the basis for high density arrays that always keep the strong carbon-carbon bonds, 

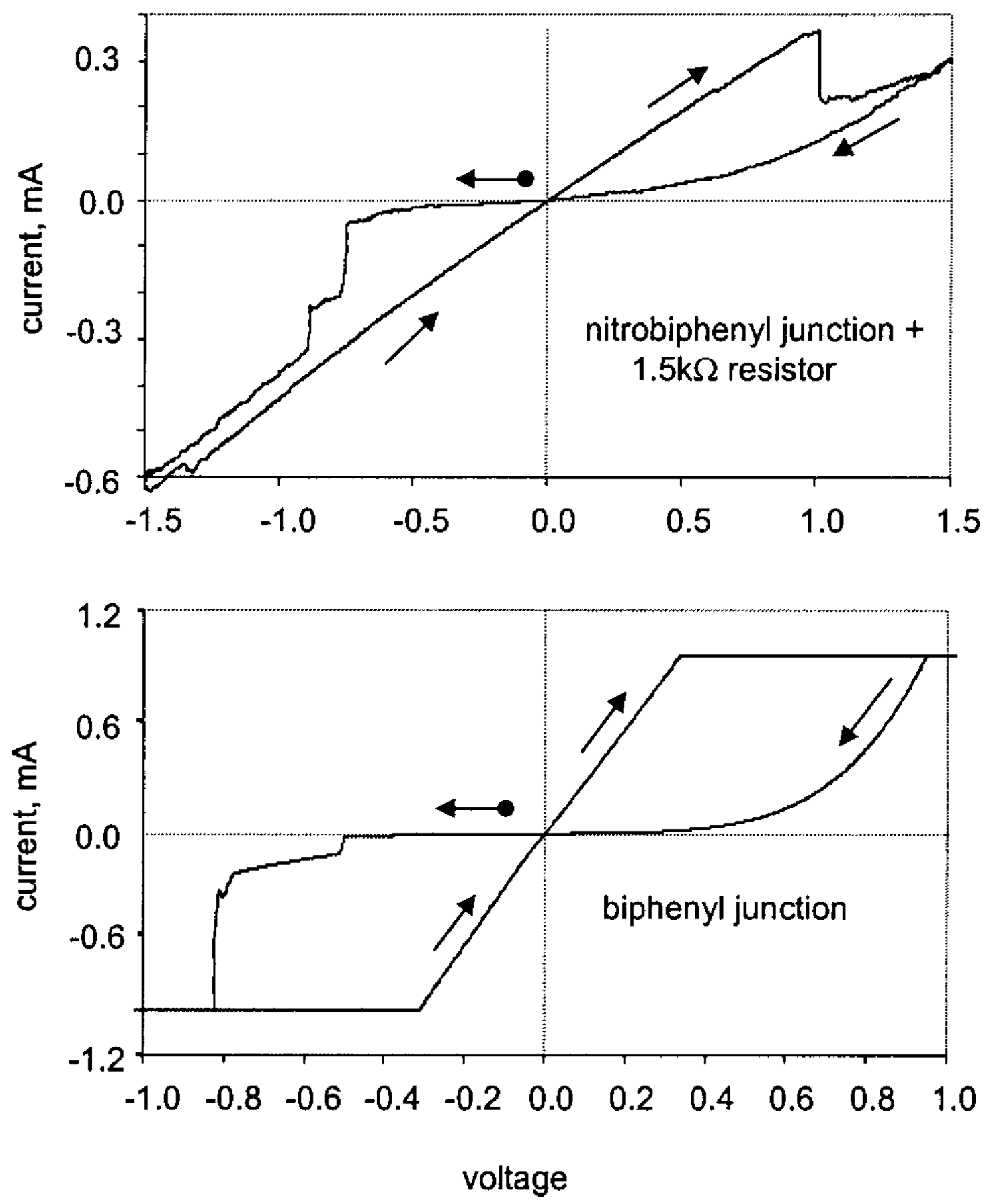

Figure 6.2: Room-temperature current/voltage behavior of nitrobiphenyl (top) and biphenyl (bottom) molecular junctions, scanned at $0.1 \mathrm{~V} / \mathrm{s}$. Arrow with circle indicates initial scan direction. The nitrophenyl junction had a $1.5 \mathrm{k} \Omega$ resistor in series to prevent overcurrent damage, and the biphenyl junction was limited to $1 \mathrm{~mA}$ by the potentiostat. Scan rate was $0.1 \mathrm{~V} / \mathrm{s}$. Reprinted with permission from the Electrochemical Society, Reference [153]. 


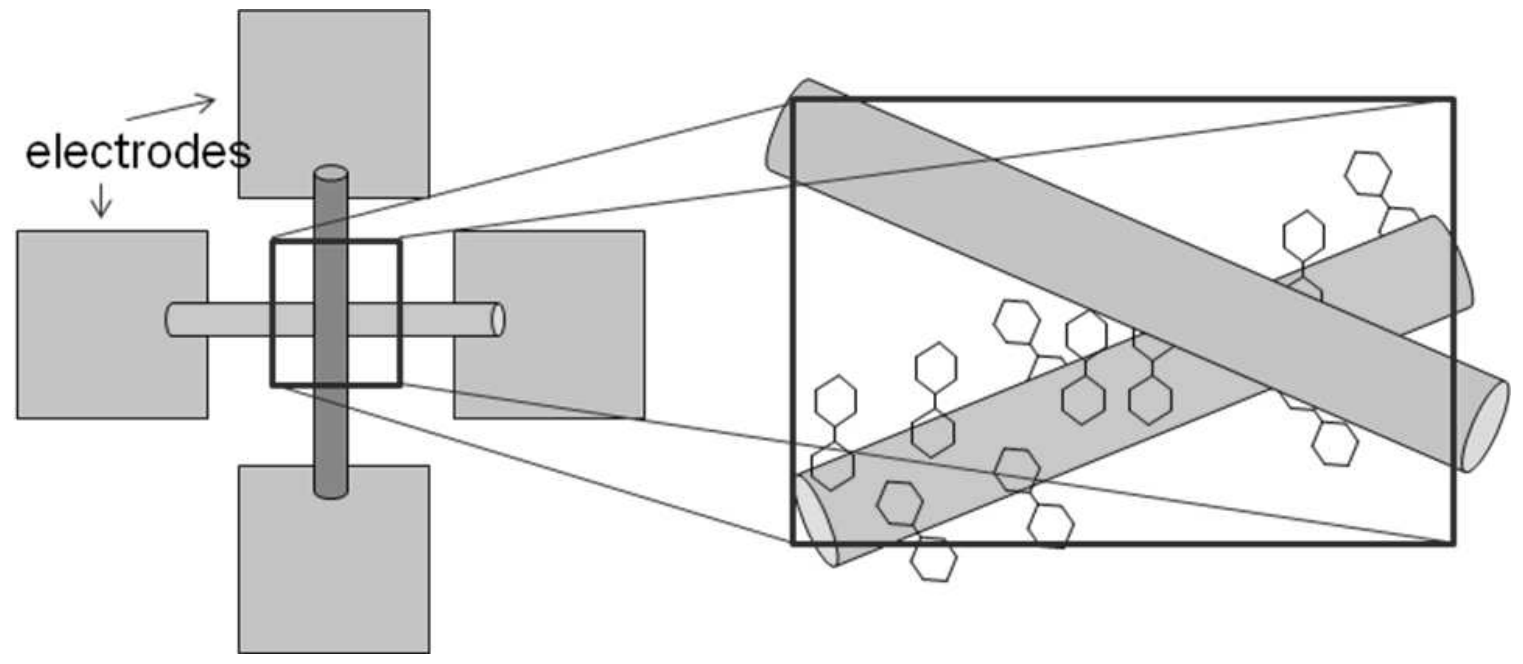

Figure 6.3: Schematic cartoon of initial junction design. The bottom CNT is functionalized and deposited via dielectrophoresis, then another dielectrophoresis deposits the second, unfunctionalized CNT normal to the first, creating a cross junction.

which should keep the device more stable than those previously reported.

This project was by far the most challenging I worked on, and required mastering the projects described in the previous chapters. In order to reach this goal, I had to master growing carbon nanotubes, functionalizing them, placing them by dielectrophoresis, designing a photomask and ultimately combining all the techniques. Our design concept was to be like one shown in Figure 6.3, and the fabrication is as follows. First, the device geometry would be patterned by photolithography on an insulating substrate, followed by pyrolysis to reduce the photoresist to a glassy carbon film. Next, a growth substrate of MWCNTs would be used as the working electrode in an electrochemical cell such that the MWCNTs would be functionalized by the irreversible reduction of biphenyl ions on the sidewalls. Then, a two-step dielectrophoresis process would take place, one with functionalized MWCNTs and one with unfucntionalized MWCNTs, so that the two species would cross, with biphenyl connecting them electronically. This junction forms a molecular bit, like the one shown in Figure 6.3, able to read and write a high or low conductance state.

However, as research progressed on each project leading up to the final device, we encountered problems that would make the device extremely difficult to make and to which no simple solutions could be found. These are discussed below. 


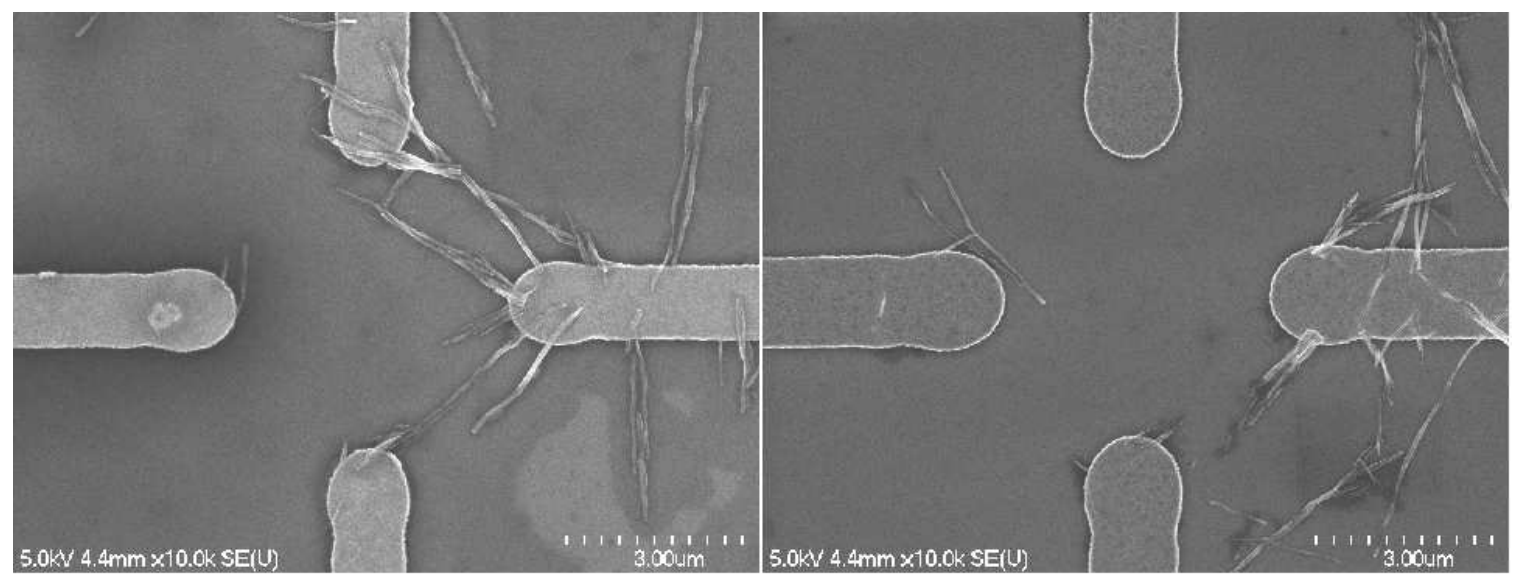

Figure 6.4: When attempting to connect opposite electrodes via dielectrophoresis in a four-electrode pattern, the tubes short to the floating potential electrodes instead of being attracted to the desired electrode.

\section{MWCNT Cross}

The MWCNT cross is what is achieved by a two-step dielectrophoretic deposition using two pairs of electrode fingers perpendicular to one another, and is a necessary component of the initial device design. The cross is, perhaps, one of the trickiest goals of the project and has two major problems. First, when using the four electrode configuration designed for a two-step cross configuration, the MWCNTs short to the floating potential electrodes and not the electrode we desire, as demonstrated in Figure 6.4. Libao An (of Dr. Friedrich's group) and I spent a good amount of time trying to implement or conceive of work-arounds for this problem (including connecting the electrodes by resistors to reduce the potential step), but came up with nothing that worked or could be simply implemented. Second, our studies indicate that for any deposition you do achieve, if a second deposition is attempted in the same device pattern it removes the first MWCNT. It would require a very large amount of work to overcome this problem. The envisioned solution is a multi-step process in which one pair of electrodes is patterned and DEP is done. Then, the same electrodes are covered with a high-dielectric material that can be removed later without damaging the tubes, but the gap remains open. The second pair of electrodes is then patterned, and dielectrophoresis takes place across them, forming the junction. Finally, the dielectric is removed. This is, however, beyond the capabilities we have at MTU at present. 


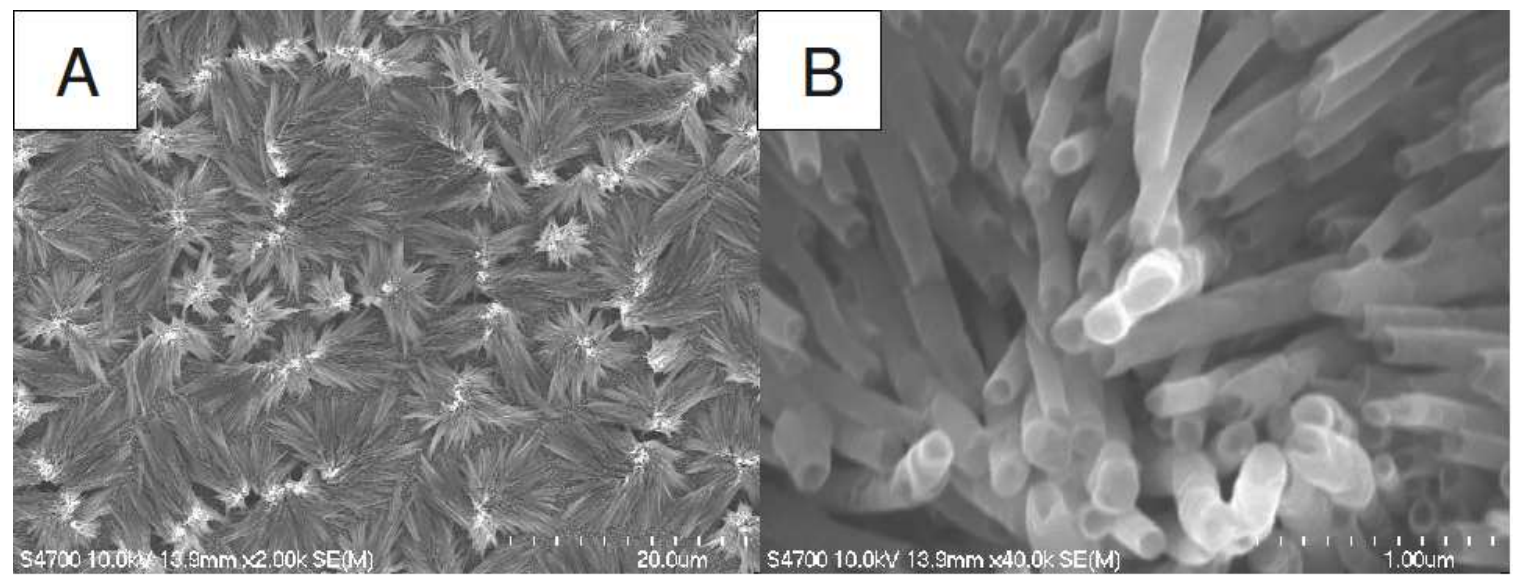

Figure 6.5: SEM micrographs of MWCNTs after immersion in liquid, showing how they collapse into large pyramids. (A) All over the substrate, the MWCNTs have collapsed, covering up most of the available CNT surface area. (B) A higher-magnification image showing how close the clumped MWCNTs are.

\section{Electrochemistry}

The electrochemistry aspect of the device design had from the beginning two major difficult-toanswer questions and one major problem. The first difficult-to-answer question is whether or not the carbon nanotubes are in sufficiently good electronic contact with the substrate to be properly biased as working electrodes. Second, if the bias is good enough, would the electrochemistry take place on the sidewalls when the opened tips contain already opened bonds that could be interacted with at a much lower energy cost? Ultimately, we never answered either of these because the major problem made them moot. When conducting the etching and dispersion experiments, we learned that MWCNTs in solution form themselves into bundles. Since the desired functionalization was to take place on the sidewalls, the vast majority of target area would be screened by the bundling action of the MWCNTs, preventing it from taking place, as shown in Figure 6.5. Even if we solved all the preceding issues, there was no guarantee the functional groups on the sidewalls would survive the several hours of ultrasonication necessary to disperse the MWCNTs. 


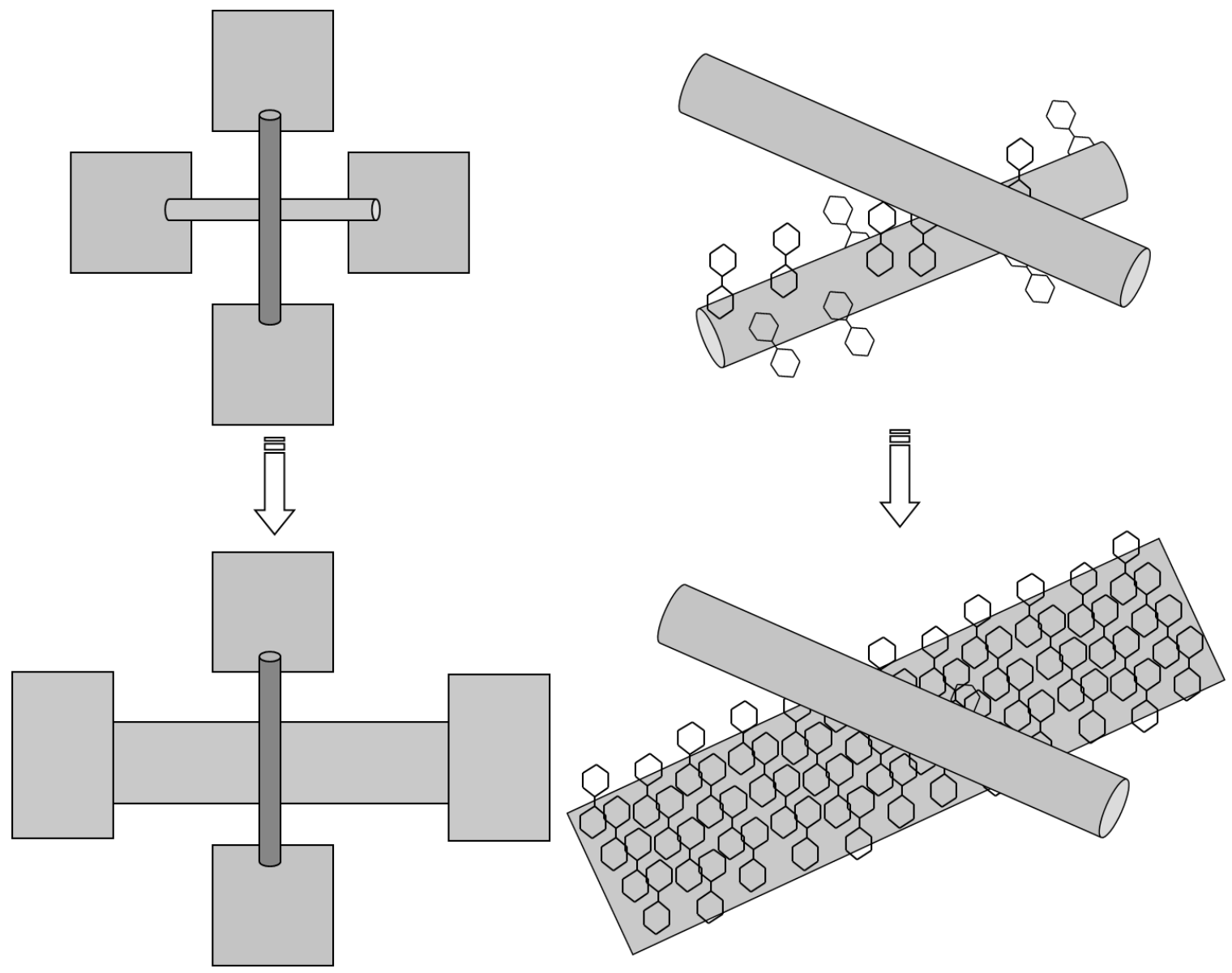

Figure 6.6: Schematic diagram showing the alterations from the previous (top) to the new (bottom) device design. In brief, one CNT has been replaced with a more easily functionalized thin film strip that also removes the need for the problematic two-step dielectrophoretic deposition.

\subsection{Design Modifications}

The experimental evidence strongly suggested the original device design was prohibitively difficult for a single dissertation project. Therefore, a new, altered design was needed that addressed the difficulties encountered thusfar. The solution I reached was to replace one half of the MWCNT cross with a photolithographically patterned electrode strip. This thin film electrode could be functionalized directly, bypassing the electrochemical problems, and would eliminate the need for a two-step dielectrophoresis procedure. The change in design is shown schematically in Figure 6.6.

This new geometry allowed us more freedom in components, while still staying true to the original 
design intent. If the device was patterned in glassy carbon, the carbon-organic-carbon device could still be constructed. However, we could also pattern the electrodes with gold and investigate a goldthiolorganic-carbon device. This second type of device is still characterized by very strong bonds, so is true to the goal of the initial design. Importantly, the new gold-thiolorganic-carbon junction can later be extended to the original device design of a nanomaterial cross-junction by using gold nanowires as one of the wires in the cross.

Design for the new device testbed pattern had the following requirements:

1. The pattern must contain a macroscopic touchpad that could be contacted with alligator clips in order to electrochemically functionalize the electrode strip.

2. The pattern must contain different sizes of features (strip width, finger dimensions, gaps) because we had no way of testing how features would behave under the pyrolysis necessary to make the glassy carbon films, and to account for the observed micron-scale variation in the photolithography process.

3. Because of the possibly large resistance, we wanted to minimize the length of 'thin' features as much as possible.

Though I had used Cadence software for previous mask design, the university had no licensed copies during this time. Therefore, the design files for the mask patterns and overall mask map were made using the freeware Electric (GNU). The testbed consists of a macroscopic touch-pad with branches extending outward in repeated patterns; the overall design of the testbed is shown in Figure 6.7. The branches are composed of two types. The first pattern type comprises roughly $2 / 3$ of the branches of the device and are shown in Figure 6.8. This pattern type is a strip flanked by two micro-touchpads with electrode fingers extending inward toward the strip. As you travel down the branch, the gap size changes, to account for different sized materials and the variance in photolithography. As you travel from strip to strip, the thickness changes, with a minimum of 2 $\mu \mathrm{m}$, stepping by 2 to a maximum of $6 \mu \mathrm{m}$ before repeating. This was to account for the fact we had no way of testing the resistance through strips of thin film without already having the pattern. The idea with these patterns is that the MWCNTs literally form a cross with the strip, and reach from one microelectrode pad to the other. 


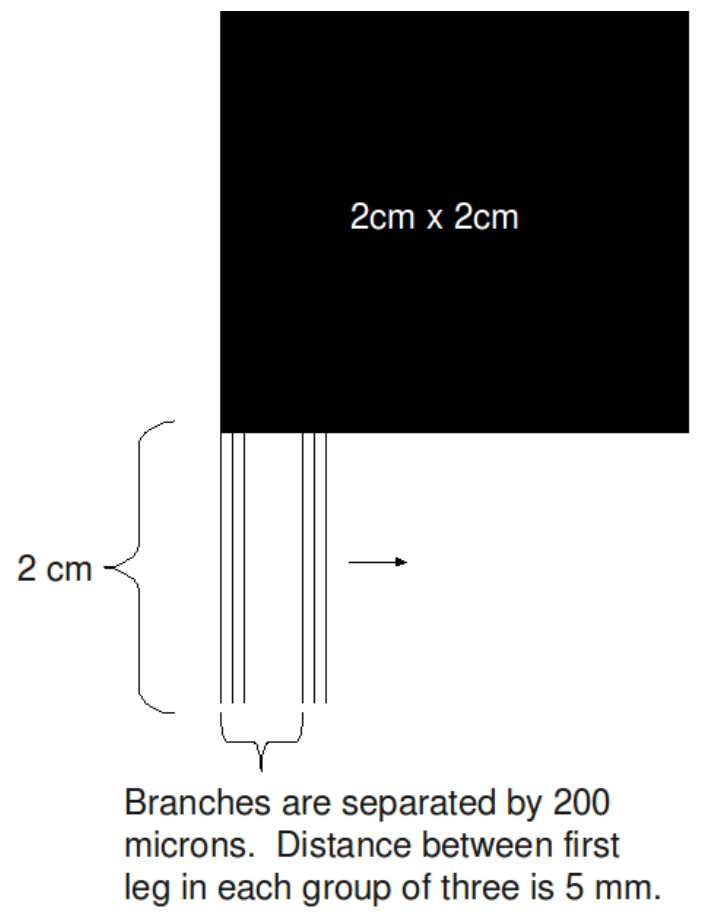

Figure 6.7: Design of the overall testbed pattern. A macroscopic touchpad is included so the branches can all be biased using electrochemistry. The branches vary in width in groups of three before repeating.

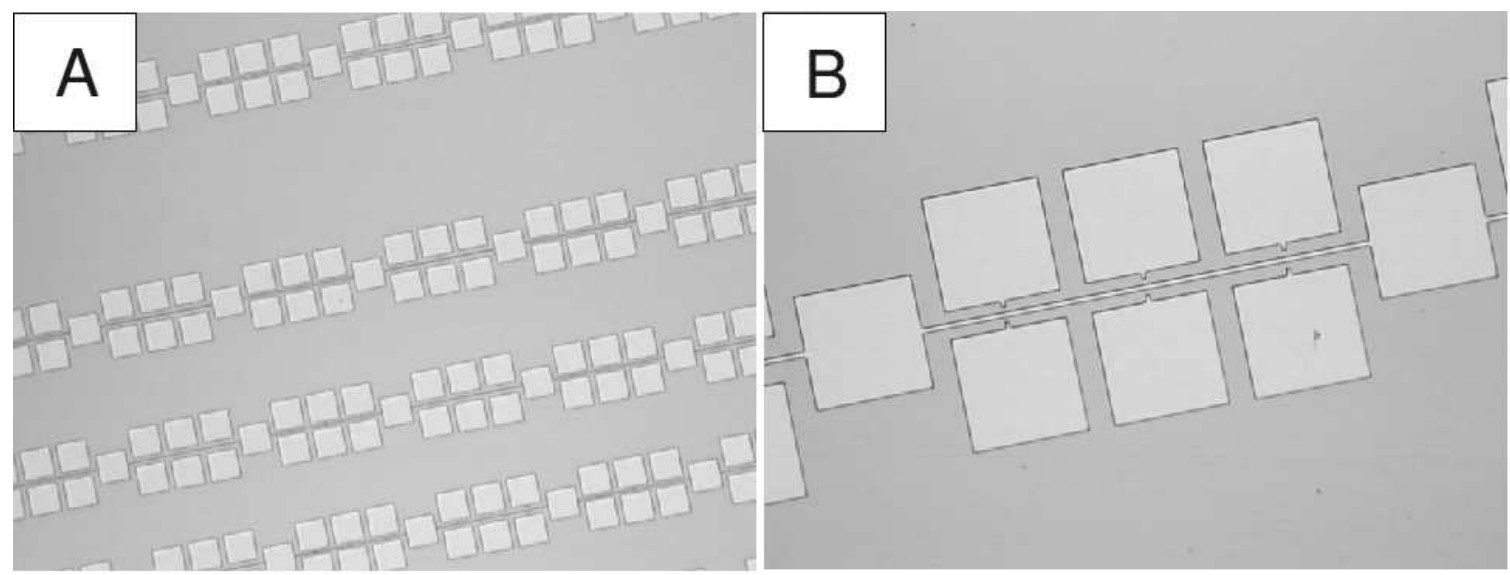

Figure 6.8: Optical microscope images of the first testbed device pattern; the large square pads are $100 \times 100 \mu \mathrm{m}$. (A) The strip "branches" are grouped in threes, with a repeated pattern of 2,4 and $6 \mu \mathrm{m}$ widths. (B) A higher-magnification image of a single branch. A series of different gap sizes from 2 to $10 \mu \mathrm{m}$ repeats after 6 gaps. 


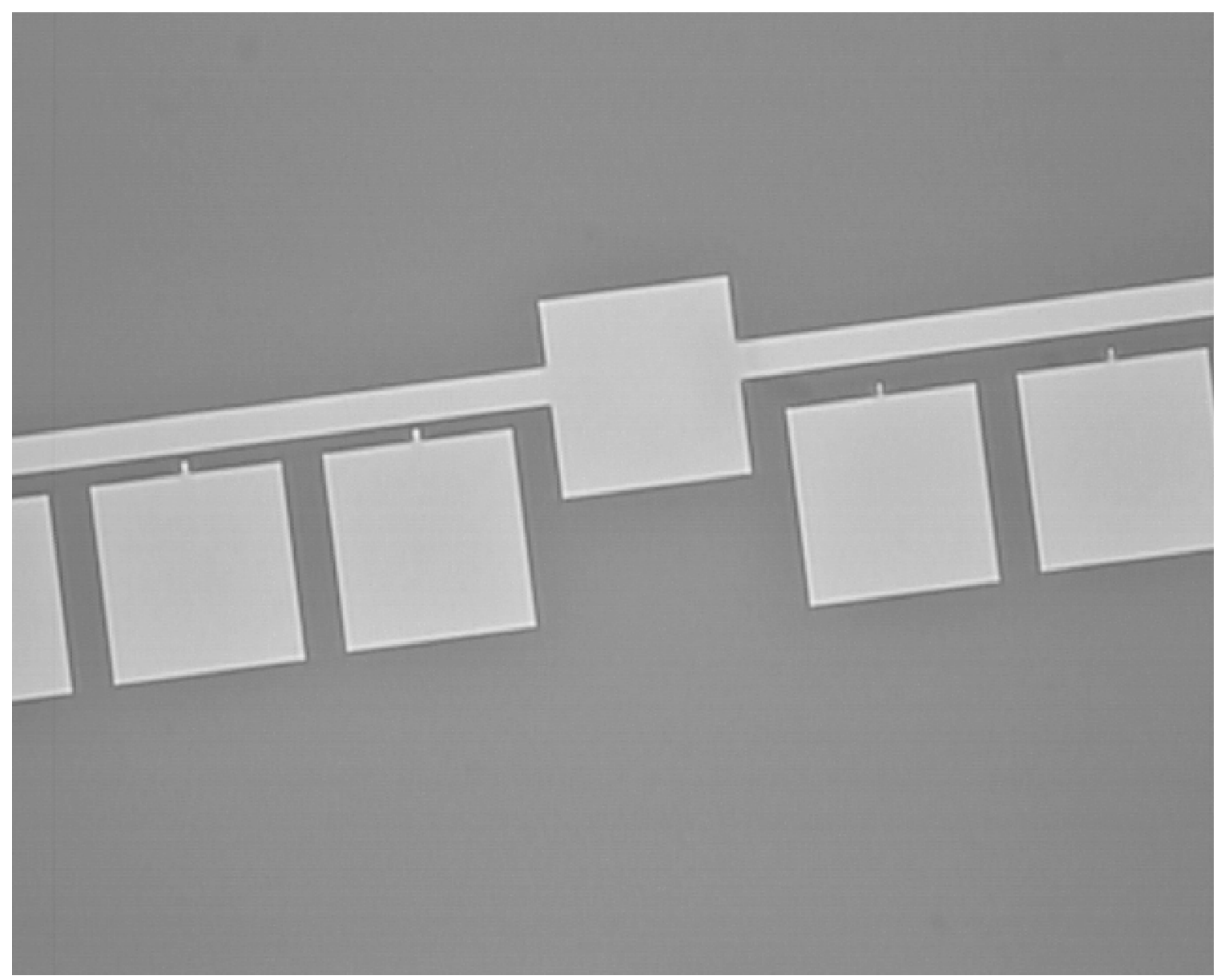

Figure 6.9: Optical microscope image of the second pattern type. The square pads are $100 \times 100$ $\mu \mathrm{m}$.

A second, simpler pattern type comprising the remaining branches was also included, with only a single microelectrode pad and a very thick strip. This geometry, shown in Figure 6.9, should be easier to use for testing, but could not be used to make an array of junctions.

\subsection{Carbon-Organic-Carbon Approach}

Both a carbon-organic-carbon and gold-thiolorganic-carbon approaches were investigated, but ultimately a decision had to be made as to which to follow, and the gold option was chose. Work done on the carbon device is detailed in this section, with gold-thiolorganic-carbon described in the 
following chapter.

\section{Glassy Carbon Electrodes}

The first requirement is patterned, atomically flat (RMS roughness $<.5 \mathrm{~nm}$ ) to serve as the electrodes. Carbon films were patterned as described in R.L. McCreery group's work, such as in Reference [154]. In brief, photoresist (such as Futurrex PR-1000A) is spin-coated for 60 seconds and 4000 rpm on to a substrate and soft-baked for 2 minutes at $120^{\circ} \mathrm{C}$. Pyrolysis was carried out in a quartz tube furnace by first flushing with the forming gas mixture (100 sccm, 95\% $\mathrm{N}_{2}+5 \% \mathrm{H}_{2}$ ) for $20 \mathrm{~min}$ at room temperature. Next, while the gas flow continued, the temperature was increased by the rate of $50{ }^{\circ} \mathrm{C} / 5$ minutes up to $1000{ }^{\circ} \mathrm{C}$. This temperature was held for $60 \mathrm{~min}$ before allowing the system to cool with the carrier gas was kept flowing until the samples cooled to room temperature. Raman spectra of the spin-coated photoresist is shown in Figure 6.10(a) and after pyrolysis in Figure 6.10(b); for comparison, Raman spectra of glassy carbon is shown in Figure 6.11.

The resulting films measured with a Veeco Dimension 3000 atomic force microscope in tapping mode, with a scan rate of $2 \mathrm{~Hz}$ and an amplitude setpoint of $80 \%$. The roughness of the films was found to fall below .5 nm, within our target range. A typical scan is shown in Figure 6.12.

With the capability of making PPFs, the next step was to make patterned electrodes from the films. The procedure was the same as the PPFs, except photolithographic exposure and development were done before the pyrolysis, leaving shaped electrodes. We didn't know if the dimensions and shapes would hold during the pyrolysis, but they did. It was also shown the patterned electrodes worked with the dielectrophoretic deposition of MWCNTs, shown in Figure 6.13. The electrode geometry and MWCNTs are ready for a device, now all that remains is functionalizing the electrodes.

\section{Electrochemical Functionalization}

The electrochemical functionalization of the glassy carbon electrodes was to be done by irreversible reduction of ionic biphenyl in solution, with the glassy carbon as the working electrode. The components of the non-aqueous cell are the glassy carbon working electrode, a $\mathrm{Ag} / \mathrm{Ag}^{+}$quasi-reference electrode, a Pt wire counter electrode, acetonitrile solvent, tetrabutylammonium tetrafluoroborate as the supporting electrolyte and bipheynl diazonium salt as the analyte. Only plastic, teflon or teflon-coated spatulas and beakers were used to prevent contamination by metals or the residuals 

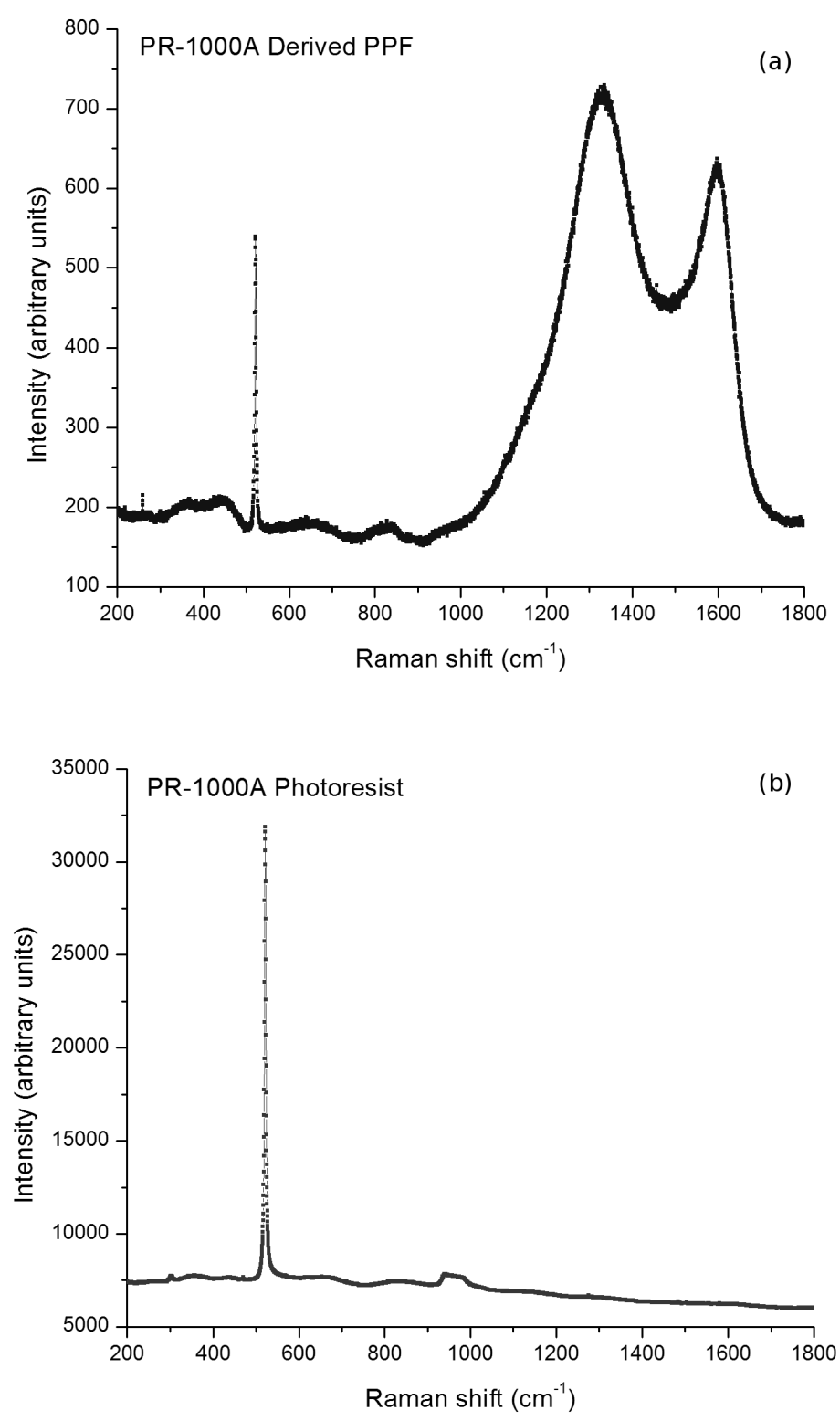

Figure 6.10: Raman spectra illustrating the formation of glassy carbon films under pyrolysis from photoresist. The peak at $520.7 \mathrm{~cm}^{-1}$ is the peak from the silicon beneath the photoresist. (a) Spectra of a PR-1000A photoresist. (b) Spectra of the pyrolyzed photoresist film. 


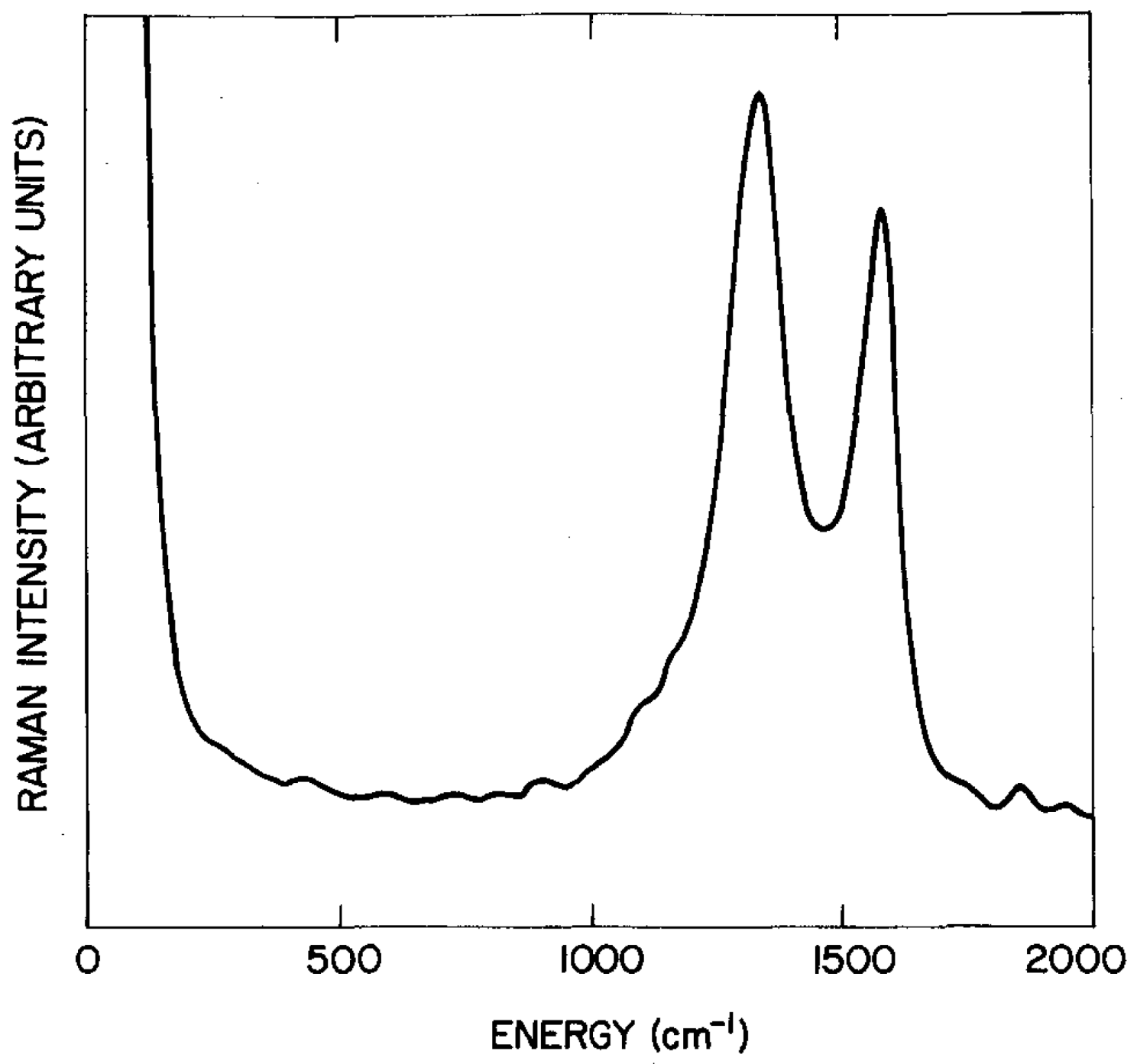

Figure 6.11: Raman spectra of glassy carbon. Reprinted with permission from Reference [155]. Copyright 1974, American Institute of Physics. 


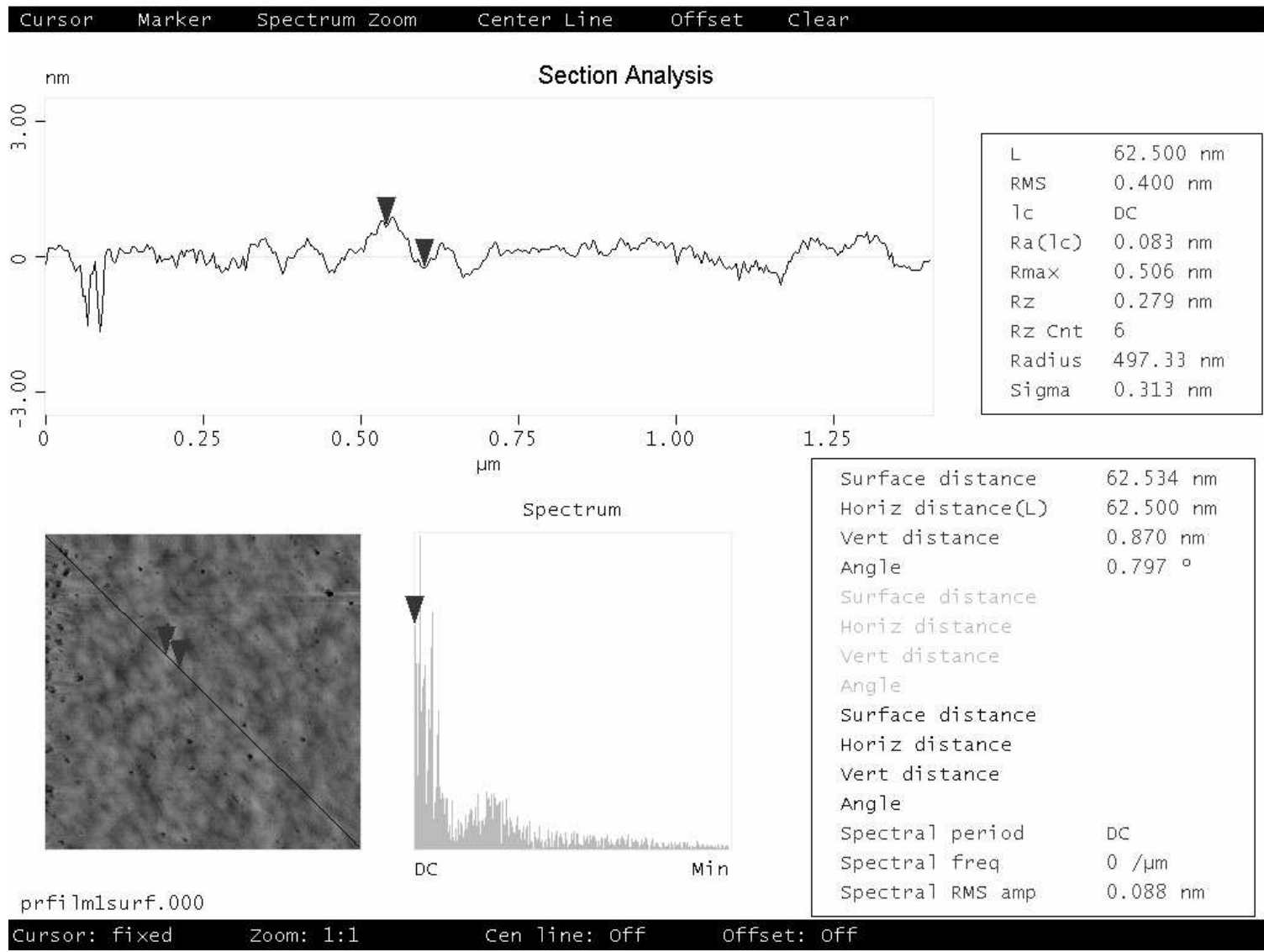

Figure 6.12: An example AFM scan of a PPF. The roughness for this line profile was .4 nm, values were generally between .1 and $.4 \mathrm{~nm}$, well within our target of below $.5 \mathrm{~nm}$. 


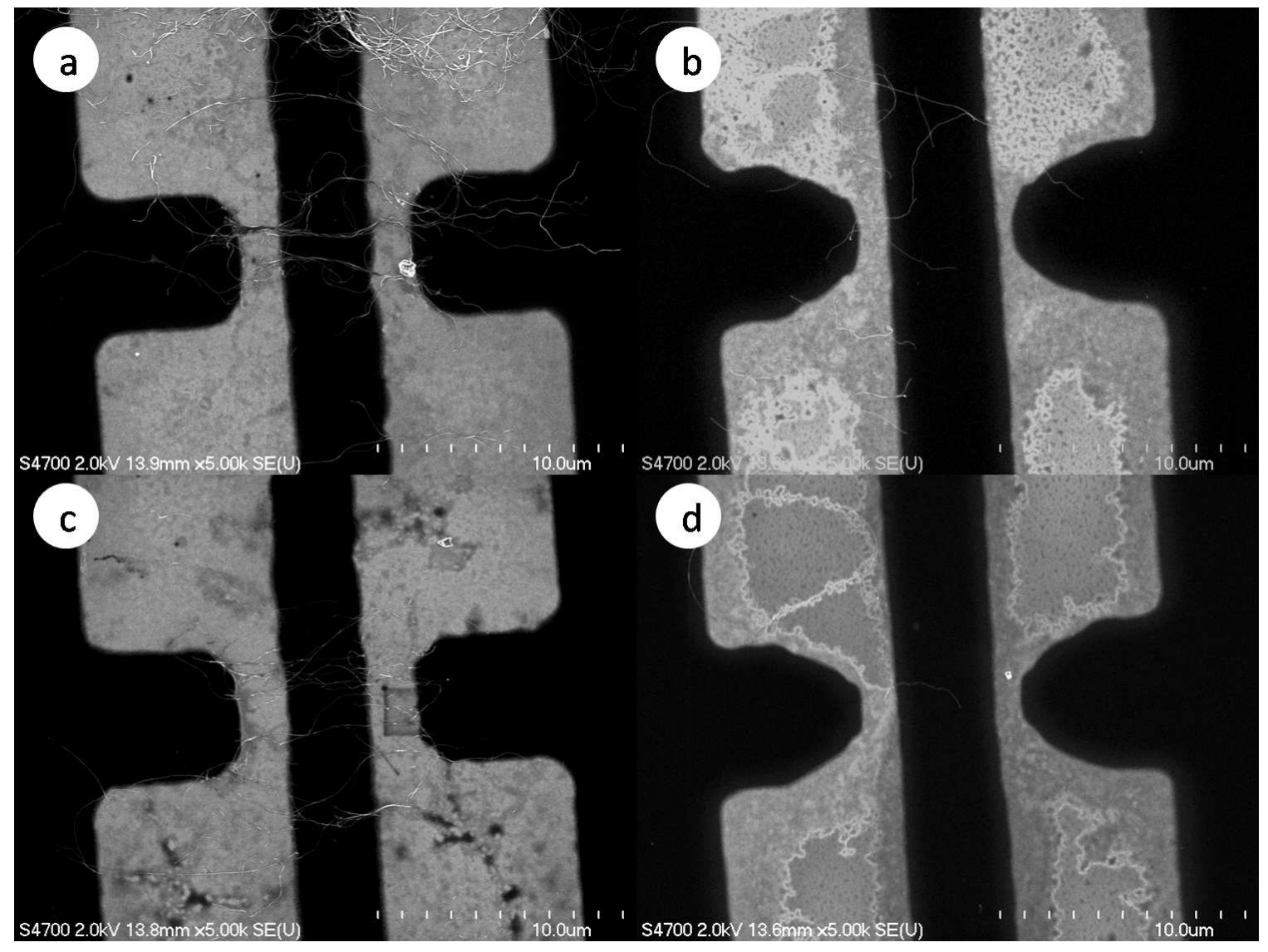

Figure 6.13: Initial tests of the strip-CNT test patterns were done using PPF as the electrode material. The solution concentration was $16 \mathrm{~mm}^{2} / 20 \mathrm{~mL}$ ethanol. Dielectrophoresis was performed at $50 \mathrm{kHz}$, with sonication time and field strength varying as follows. (a) $2 \mathrm{hrs}, 32 \mathrm{~V}_{p p}$, (b) $2 \mathrm{hrs}$, $3.2 \mathrm{~V}_{p p}$, (c) $3 \mathrm{hrs}, 32 \mathrm{~V}_{p p}$, (d) $2 \mathrm{hrs}, 3.2 \mathrm{~V}_{p p}$. 


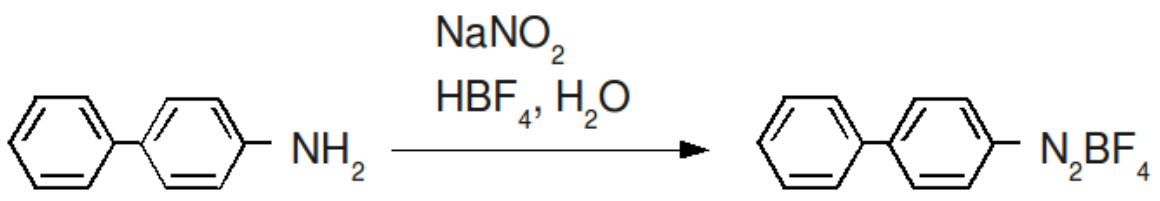

\section{In solution, it then breaks into the aryl diazonium ion and $\mathrm{BF}_{4}$ :}

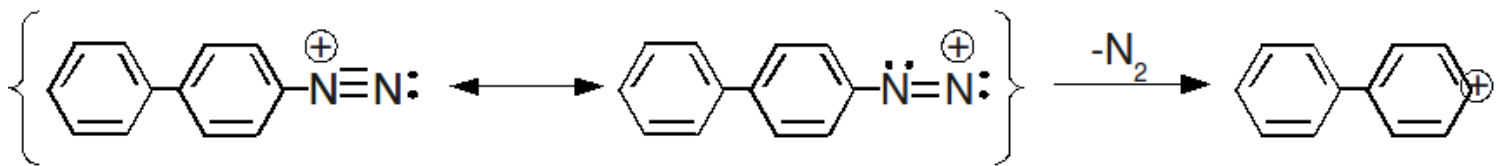

Figure 6.14: Steps of biphenyl diazonium salt synthesis. The process ends as the salt decays into a biphenyl ion in solution.

caused by HF in the glass.

The $\mathrm{Ag} / \mathrm{Ag}^{+}$quasi-reference electrode was prepared by dissolving $10 \mathrm{mM}$ of $\mathrm{AgNO}_{3}$ in $0.1 \mathrm{M}$ n-tetrabutylammonium tetrafluoroborate $(\mathrm{TBABF} 4)$ in acetonitrile. The solution was placed in a nitrogen environment glovebox and was deaerated for 10 minutes by bubbling nitrogen. After deaeration, it was dispensed into the electrode casing using a plastic syringe, before inserting the silver wire and cap. The reference is stored in deaerated $0.1 \mathrm{M}$ TBABF4 in acetonitrile. The reference and $\mathrm{Pt}$ wire counter electrodes were purchase from CHI.

The derivatization is performed as outlined as in References [156] and [157].

1. Samples are cleaned with acetonitrile.

2. Derivatization is performed using $1 \mathrm{mM}$ solutions of the biphenyl diazonium salt $\left(\mathrm{C}_{6} \mathrm{H}_{5} \mathrm{C}_{6} \mathrm{H}_{4} \mathrm{~N}_{2} \mathrm{BF}_{4}\right)$ in $0.1 \mathrm{M}$ TBABF4 in acetonitrile.

3. The diazonium salt solution was deaerated with nitrogen for at least $10 \mathrm{~min}$ before derivatization.

4. The electrodes were introduced to the solution, forming the electrochemical cell.

5. Derivatization of the surfaces was performed using cyclic voltammetry with a scan rate of 200 $\mathrm{mV} / \mathrm{s}$ for one cycle between +0.4 and $-0.8 \mathrm{~V}$ versus $\mathrm{Ag} / \mathrm{Ag}+$.

6. The samples were rinsed with acetonitrile afterward. 
The diazonium salt needs to be derived by procedures detailed in Reference [156]. The steps are as follows:

1. $0.5 \mathrm{~g}$ of the amino precursor (4-aminobiphenyl. $\mathrm{C}_{12} \mathrm{H}_{11} \mathrm{~N}$ ) was weighed into a three-necked 50 $\mathrm{mL}$ round bottom flask.

2. $10 \mathrm{~mL}$ of $50 \%$ fluoroboric acid $\left(\mathrm{HBF}_{4}\right)$ was added and the mixture was stirred with a magnetic stirring bar.

3. A 3:1 molar ratio of $\mathrm{NaNO}_{2}$ (relative to the amino precursor) was weighed in a separate container and just enough water was added to dissolve it at room temperature.

4. A thermometer was inserted into the three-necked flask and both solutions cooled to $0{ }^{\circ} \mathrm{C}$ by sitting in an ice bath.

5. The cold $\mathrm{NaNO}_{2}$ was added dropwise, always keeping the temperature below $4{ }^{\circ} \mathrm{C}$.

6. After all the $\mathrm{NaNO}_{2}$ is added, the mixture is stirred for $\sim 30$ minutes.

7. The insoluble diazonium salt was filtered using a Buchner funnel.

8. Very quickly, anhydrous ether is used to remove the sediment from the round bottom flask and is likewise filtered.

9. The filtered material is recrystallized by dissolving in $0{ }^{\circ} \mathrm{C}$ acetonitrile followed by slow addition of cold anhydrous ether.

10. Filter one more time, then use immediately.

Representative derivatization scans at $0.2 \mathrm{~V} / \mathrm{s}$ are shown in Figure 6.15 for films and Figure 6.16 for device testbed patterns. Both scans show irreversible processes, which indicates that the desired process is taking place. As expected, the level of current is lower for the device pattern since it has less biased surface available for redox reactions.

\subsection{Junctions}

Though the cyclic voltammagrams from the derivitization are consistent with what we expect (an irreversible process), the I-V characteristics of the tested junctions are not. FTIR shows no sign of 


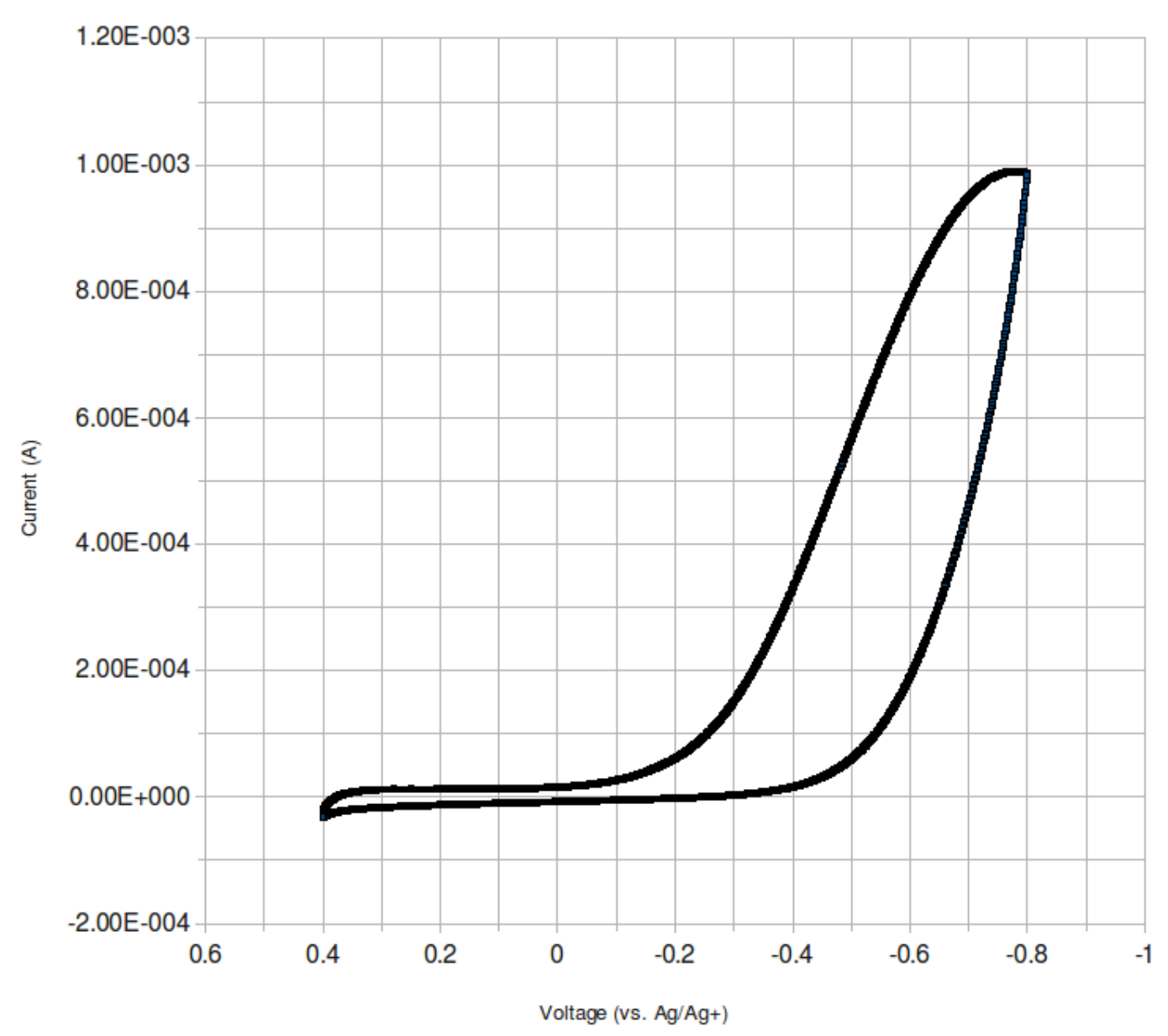

Figure 6.15: A representative derivatization scan for a PPF film at $0.2 \mathrm{~V} / \mathrm{s}$. The curve indicates an irreversible process. 


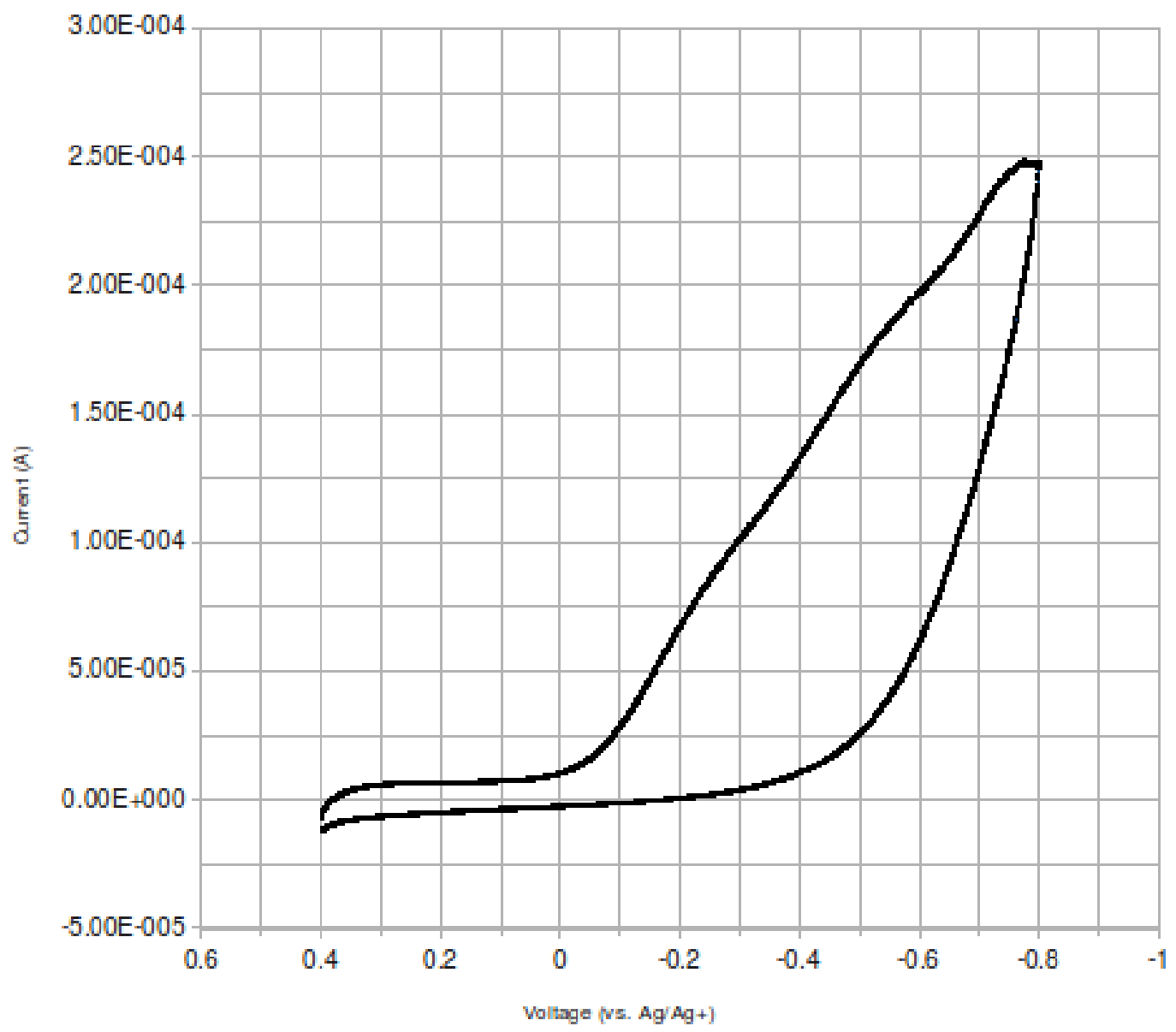

Figure 6.16: A representative derivatization scan for a PPF device testbed pattern at $0.2 \mathrm{~V} / \mathrm{s}$. The curve indicates an irreversible process. 
biphenyl and STM is not conclusive; the STM never displayed the expected switching characteristics of biphenyl, sometimes showed rectification and sometimes just showed open or shorted circuits. These curves showed no trend, the first scan at a given point could be any of the curve types and each point would cycle through a few of the curves in a seemingly random order. Since our experience with STM was new, samples were sent to the Army Research Laboratory in Aberdeen Proving Ground for independent testing by Mark Griep; they observed the same phenomenon (examples or some STM scans are shown in Figure 6.17. This is not enough to rule out that biphenyl is present, however. The observed non-stable behaviour could be due to biphenyl, but the results are inconclusive.

Most telling, however, is that when the PPFs are tested by making a mercury drop top contact (a molecular junction of glassy carbon-biphenyl-mercury), or the devices are tested with MWCNTs, the curves always show completely linear, ohmic contact, not the expected switching behaviour or any of the other curve shapes observed by STM. One possibility is the biphenyl monolayer is present, but not fully formed. If this were the case, the mercury would surely short to the glassy carbon layer and give us linear, ohmic curves. Another possibility is that the CV curves are not showing what we think they are. Since we have no reference CV curves, we do not know for certain if the reaction taking place is what we think it is, so it is possible that the irreversible reaction we observed was not the one we desired. All experiments were repeated several times with the same result. After extensive literature searches, the experiments were run again with even more stringent steps taken to avoid contamination by electroactive particles, but there was no change. While we were investigating the problems with our technique, we were also investigating Au-ODT-MWCNT junctions and, when time became scarce, we decided to focus on the those junctions. The Au-ODT-MWCNT option was chosen because we believed we had a higher chance of making complete monolayers within the allotted time. However, once whenever the problem with the BP device is identified, every piece is already in place.

\subsection{Summary}

The original concept of the molecular junction device needed to be revised to account for problems we learned about as we progressed toward being able to fabricate them. The new device uses a strip of functionalized thin film as the bottom electrode and a MWCNT as the top. The new geometry allows 

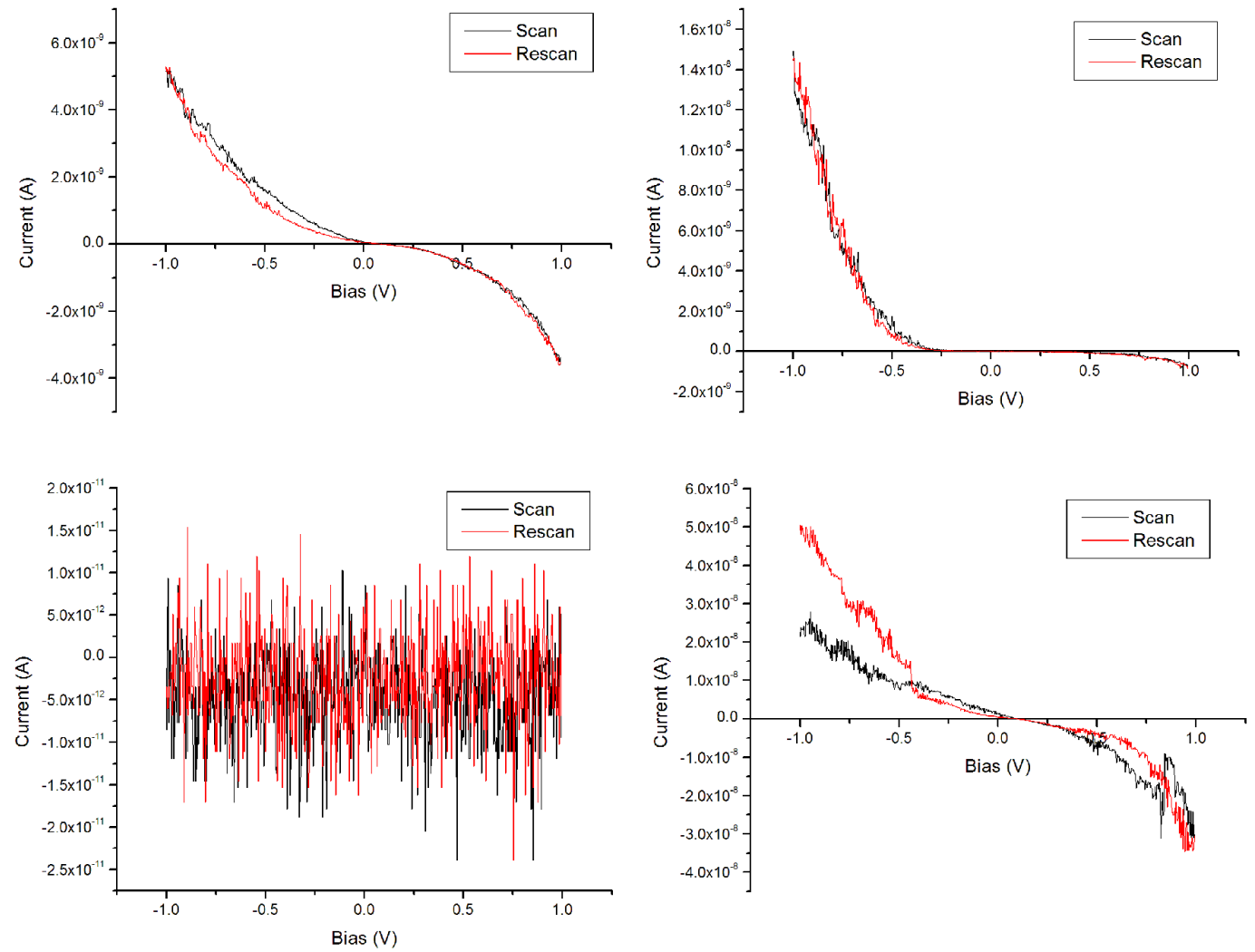

Figure 6.17: STM of biphenyl functionalized carbon films. None of the scans showed the expected switching character of biphenyl. Sometimes the films showed weak hysteresis, sometimes not. Sometimes the film responded like an insulator with an open circuit showing, sometimes showing rectification. 
us to test Au-thiolorganic-MWCNT junctions as well as glassy carbon-organic-MWCNT junctions. In both cases, we keep the key design components of strong bonds and scalability.

The MWCNT-biphenyl-MWCNTs junctions were fabricated, but continue to show ohmic behaviour and not the expected high and low current states. An ongoing investigation as to why it is not behaving as expected is underway, but ultimately we chose to spend our remaining time on the Au-ODT-MWCNTs because we thought it was more likely to work within the time remaining for the project. 


\section{Chapter 7}

\section{Molecular Electronics Devices II}

This chapter discusses the work done to develop a fabrication technique for making molecular junctions between gold and carbon nanotube contacts. Because the device geometry, as shown in the previous chapter, was designed as it was as a single step fabrication process, the strip and electrodes will all be gold, so the actual junction studied will be $\mathrm{Au} \rightarrow$ molecule $\rightarrow$ MWCNT $\rightarrow$ molecule $\rightarrow$ $\mathrm{Au}$; this can easily be changed by a two-step photolithography pattern so the electrode fingers are not gold, while the strip is.

\subsection{Self-Assembled Monolayers}

First introduced by Zisman in 1946 [158], self assembled monolayers (SAMs) are self-forming films made on solid surfaces by specially-tailored surfactants. The films form by spontaneous chemical synthesis at the surface that slowly moves toward an equilibrium state $[159,160]$. The surfactants used to form the monolayers are designed so that one end has specific, favourable interaction with the surface of interest [160]. In the 1980's, work by Nuzzo and Allara introduced the thiol-Au systems [161], one of the most popular SAM systems in use today, particularly alkanethiols, because they represent an easy path to linking inorganic and organic materials [162].

Each surfactant molecule can be divided into three parts, shown schematically in Figure 7.1(a): that is the head (linking) group, the backbone (main chain) and the terminal specific (active) group [162]. Monolayers can be deposited by vapor or solution phases [159, 160, 162]. A simplified picture 


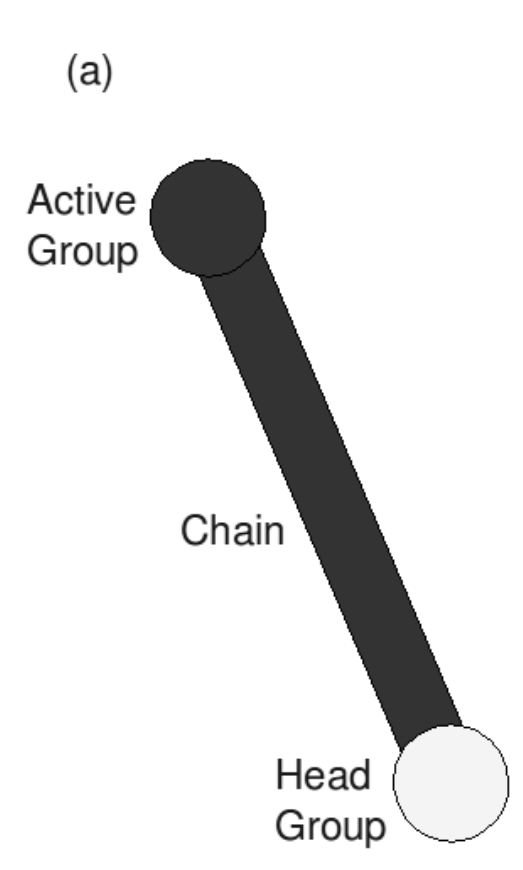

(b)

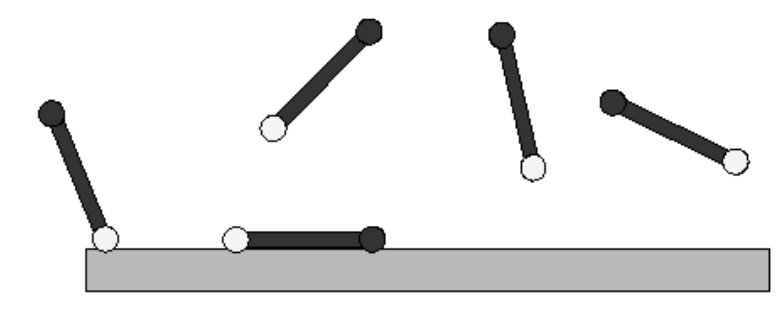

(c)

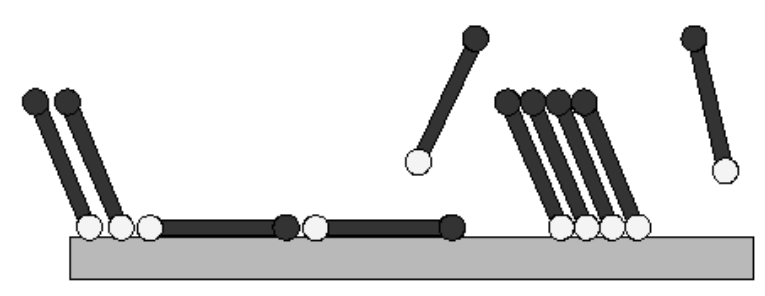

(d)

Figure 7.1: Cartoon showing the SAM molecule and deposition process. (a) A representation of a SAM molecule showing the three vital parts: head, chain and active group. (b) SAM deposition begins as SAM molecules are transported to the targeted surface. (c) Adsorption takes place, forming nucleation sites. (d) Over time, the nucleation sites grow and the molecules rearrange until a dense equilibrium structure is formed.

of the assembly process is shown in Figure 7.1(b)-(d) and is described as follows. Initially, the molecules transport to the solid surface. Next, the head groups begin specifically adsorbing to the target surface. Finally, nucleation sites of close-packed molecules continue to grow until a dense equilibrium film structure is formed $[159,162]$.

\subsection{Octadecanethiol}

The molecule chosen to test our junction is called octadecanethiol (ODT), $\left(\mathrm{CH}_{3}\left(\mathrm{CH}_{2}\right)_{16} \mathrm{CH}_{2} \mathrm{SH}\right.$ ), whose thiol head group will bind strongly (40 kcal/mol [159], for comparison, C-C is $83 \mathrm{kcal} / \mathrm{mol}$ ) to the $\mathrm{Au}$ electrode surface, and whose terminal -CH will interact with the MWCNT wire laying atop it. ODT was chosen because it is a robust monolayer capable of linking metal films to MWCNTs, 
however, with some reported exceptions [163], it does not exhibit I-V characteristics that are directly useful as an electronic component; though molecular scale insulation is important to circuit design. ODT has a HOMO-LUMO gap of $\sim 6.033 \mathrm{eV}$ so the monolayer should behave as an insulator [164, 165] with current flow coming from tunneling through the junction [166]. If we can show the junctions retain the insulating characteristics of ODT, we can then swap ODT with another specifically-tailored SAM molecule for specific applications [164].

ODT should bond to the Au surface via the reaction [159]:

$$
\mathrm{R}-\mathrm{S}-\mathrm{H}+\mathrm{Au}_{n}^{0} \rightarrow \mathrm{R}^{-\mathrm{S}^{-}} \mathrm{Au}^{+} \cdot \mathrm{Au}_{n}^{0}+\frac{1}{2} \mathrm{H}_{2}
$$

ODT is $27 \AA$ long [167], and studies have shown the lattice constant of a monolayer on $\mathrm{Au}(111)$ surfaces is 4.6 - $4.7 \AA$, and the alkyl chains show almost no tilt (the axial direction is almost perpendicular to the substrate surface)[159]. The most commonly used Au substrates for SAMs are single-crystal, or evaporated thin films on glass or mica $[160,162]$, however our substrates with with single crystal silicon, with a thermally evaporated $\mathrm{SiO}_{2}$ layer of $120 \mathrm{~nm}$.

To form the ODT monolayer, gold deposited substrates are immersed in 2mM of ODT solution, and kept in a nitrogen environment glove box for $48 \mathrm{hrs}$. The samples were then rinsed with ethanol to remove any physisorbed overlayers and are dried in nitrogen. When in storage, the samples are kept in the nitrogen environment.

\subsection{STM Tests}

Before moving on to patterned junctions, ODT monolayers were examined by a scanning tunneling microscope (STM) in order to determine (1) if the ODT deposition was successful and (2) what the characteristic I-V curves look like. This work was carried out by Abhishek Prasad and Ravi Chintala with my assistance.

Gold films of $100+\mathrm{nm}$ thickness were deposited by PLD for the control and SAM substrates. The I-V was measured using a Veeco Dimension 3000 AFM with an STM add-on. The measurement parameters are summarized in Table 7.3.

Images of the films were obtained in a constant-current mode with a tunneling current of $(0.8 \mathrm{nA}$ $1 \mathrm{nA}$ ) to ensure no tip-induced changes were observed and the images obtained were free of artifacts 
Table 7.1: Summary of STM measurement parameters

\begin{tabular}{|l|l|}
\hline Current Setpoint: $800 \mathrm{pA}$ & Tip Bias: $500 \mathrm{mV}$ \\
Integral Gain: 0.5 & Proportional Gain: 0.7 \\
Scan Rate: $2 \mathrm{~Hz}$ & \\
\hline
\end{tabular}

before measuring I-V curves. The gold films were electronically connected to the STM stage by carbon tape, adding additional resistance on the order of $300 \mathrm{k} \Omega$ to the circuit [167]. The stage applies the bias voltage, while the tip serves as a ground. The I-V curves are shown in Figure 7.2.

It is clear that the current levels are much higher without ODT present, Figure 7.2. When we look to the curve for the ODT sample, we see wide region centered around 0 bias voltage $(\sim \pm 1.5 \mathrm{~V})$ in which almost no current is flowing (the current gap), and outside of which the current rises rapidly. This was result was consistent over different scans and samples, and consistent with other reports [165], so now we are satisfied that the SAMs are present and that the observed I-V curves are characteristic of ODT.

\subsection{Initial Patterned ODT Junctions}

The next step was to start fabricating and testing junctions. The design junctions tested here will be double junctions, meaning that the current passes through an ODT monolayer twice, as shown in Figure 7.3. Since we are currently incapable of a precise two-step deposition, this is a necessary evil for now. SEM of an actual junction is shown in Figure 7.4.

The electrodes were patterned by a photolithographic process using the strip device pattern discussed in the previous chapter. First, HMDS followed by photoresist (Futurrex PR-1000A) was spin coated at $4000 \mathrm{rpm}$ for 1 minute on a $\mathrm{SiO}_{2}$ surface and baked for 1 minute at $120{ }^{\circ} \mathrm{C}$. The resist was exposed along with a photomask, and developed in a 2:1 developer (MF 319):deionized water and open windows were electrodes will be. A film of $20 \mathrm{~nm} \mathrm{Cr}$ and $40 \mathrm{~nm} \mathrm{Au}$ was deposited by plasma sputtering, then the resist was lifted off by ultrasonication in acetone followed by ethanol, leaving only the desired electrode geometry. $\mathrm{Cr}$ is generally deposited beneath gold films on $\mathrm{SiO}_{2}$ in order to give the gold film better adhesion. Following liftoff, the ODT SAM was deposited over a 48 hour period in a nitrogen environment glovebox. After rinsing ethanol to remove excess condensate, 

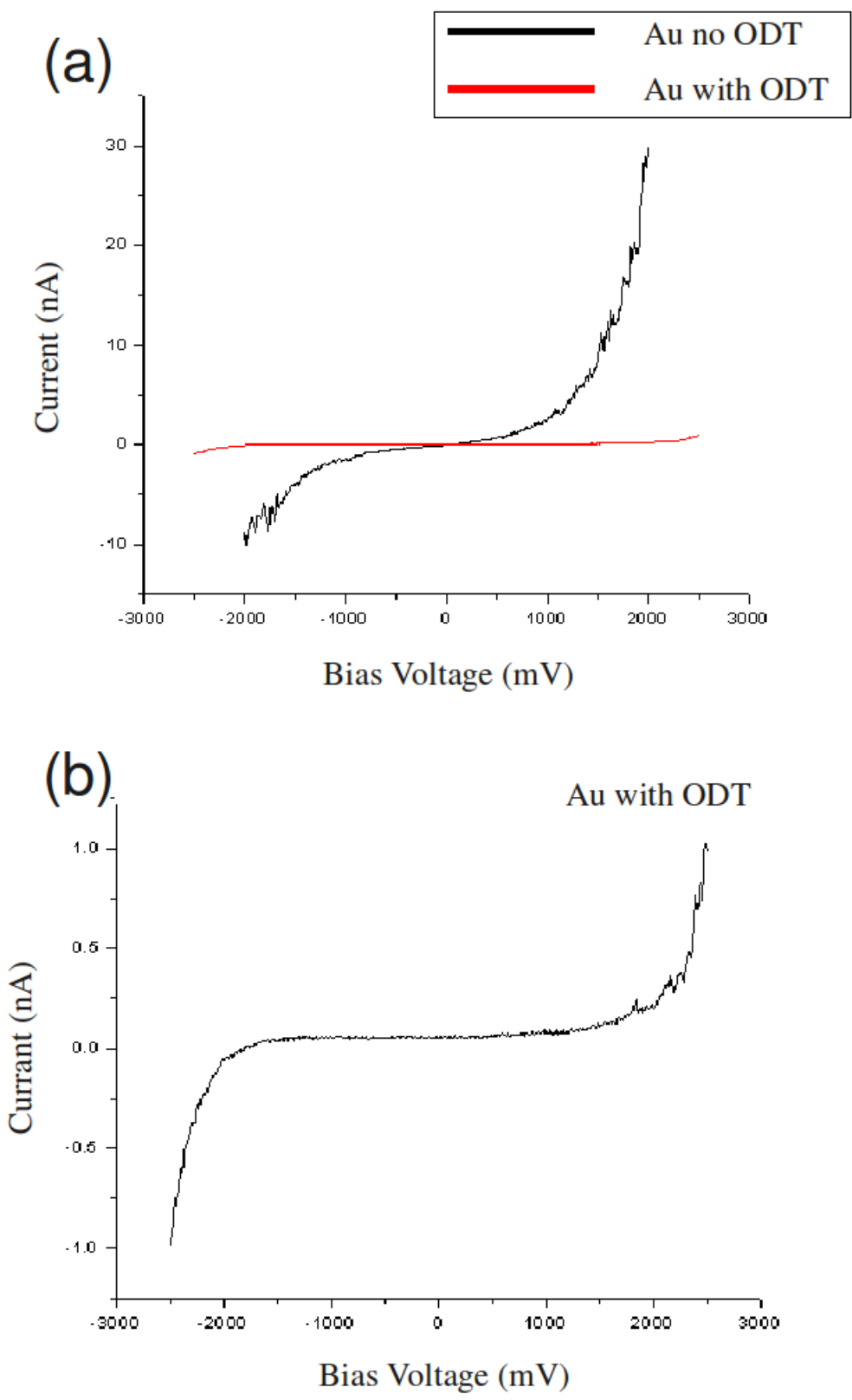

Figure 7.2: (a) Comparison of STM I-V curves of gold films on silicon substrates without (lower resistance) and with (higher resistance) ODT SAM. (b) The I-V of the ODT substrate showing the characteristics of ODT, namely much higher resistance and a region centered around 0 bias showing little to no current flow. Reprinted with permission from reference [167]. 


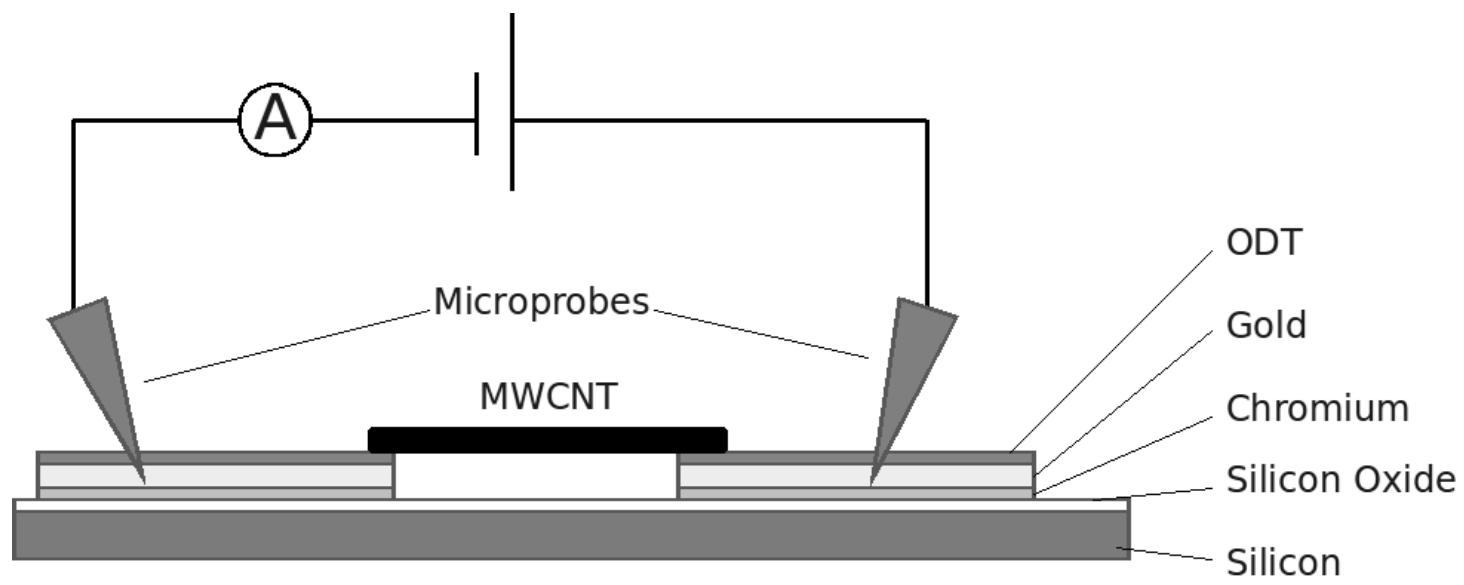

Figure 7.3: Schematic profile of the ODT double junction device. Current flow from the first probe $\rightarrow$ gold $\rightarrow$ ODT $\rightarrow$ MWCNTs $\rightarrow$ ODT $\rightarrow$ gold $\rightarrow$ second probe.

the junctions were completed by using a dielectrophoresis (as described in the relevant chapter) of T-CVD MWNCTs at $30 \mathrm{~V}_{p p}$ and $2 \mathrm{kHz}$. Testing of the devices was done with a Keithley $4200 \mathrm{SCS}$ and a microprobe system at ambient conditions.

For those junctions tested, every completed junction (ones with MWCNTs spanning connecting the electrodes, as in the left side of Figure 7.4) displayed I-V characteristics like those shown in Figure 7.5. Not a single completed junction showed any of the character we expected to see from the ODT monolayer. Interestingly, junctions that were tested and were later shown by SEM to be incomplete, or open, showed curves like those shown in Figure 7.6. These curves were repeatable, did not depend on polarity, remained after the probes were lifted and recontacted and looked different than a completely open circuit. The curves were shown to correspond to the condition of when the electrode fingers were within $2 \mu \mathrm{m}$ of the strip (Figure 7.4 right side and 7.6(a)), or $3 \mu \mathrm{m}$ (Figure 7.6(b)) of the strip. 10 and $8 \mu \mathrm{m}$ were open circuits, and the other distances were not tested. The 2 and $3 \mu \mathrm{m}$ gaps were later tested by control devices with identical fabrication but without the presence of ODT; the I-V showed an open circuit.

Junctions were also tested using the dielectrophoresis patterns. Like the other junctions, these simply showed ohmic behaviour (Figure 7.7), indicating the MWCNTs were shorting to electrodes without having to pass through the ODT layer.

We were, at this stage, certain of two things. First is that the ODT is present and is responsible 


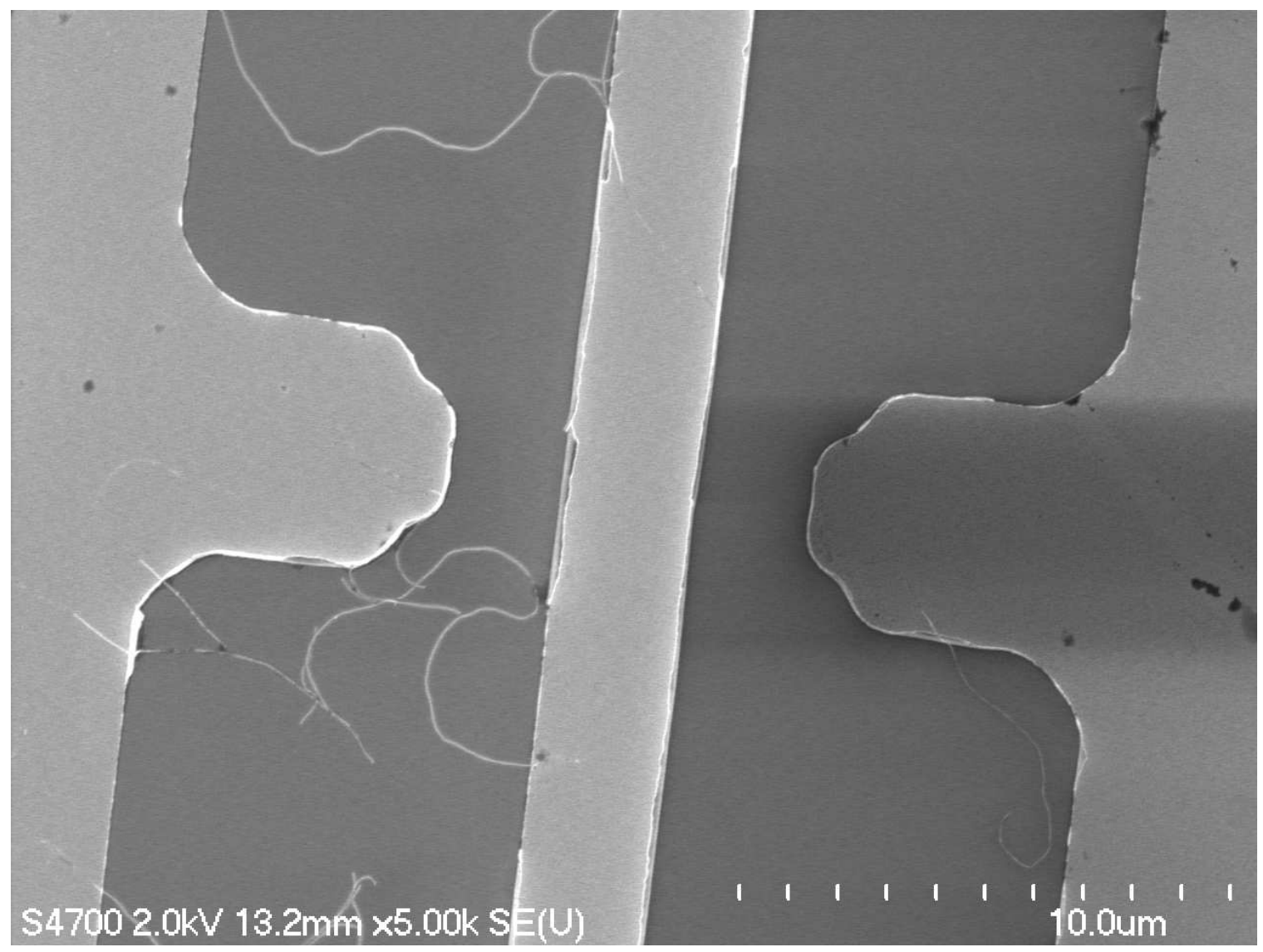

Figure 7.4: SEM of fabricated junction. The left side is a completed junction whose current is shown in Figure 7.5. The right side is open, and its I-V is shown in Figure 7.6(a). 


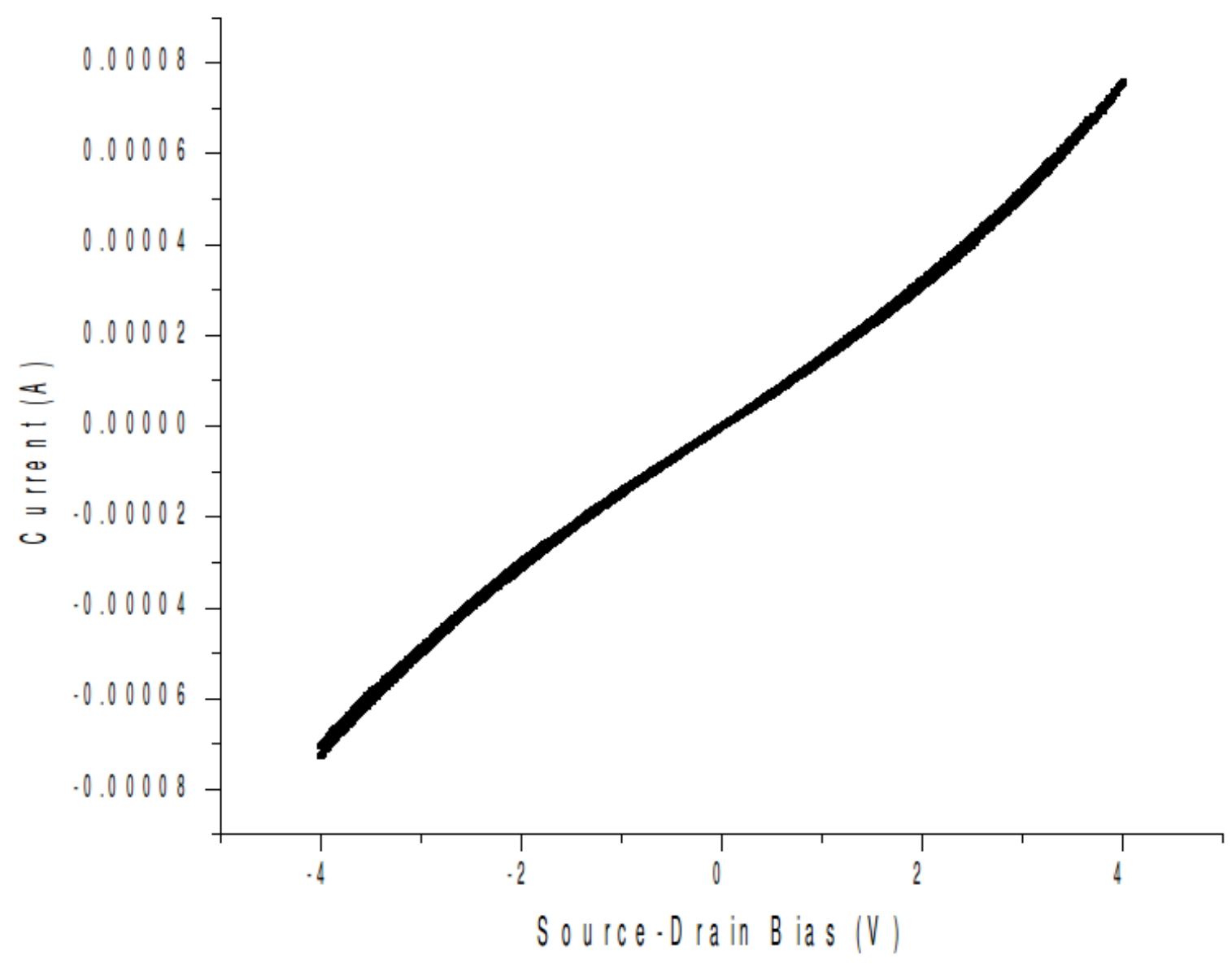

Figure 7.5: Representative I-V of initial junctions. For all tested junctions that were verified later by SEM to have MWCNTs completing the device, the current had this shape. 

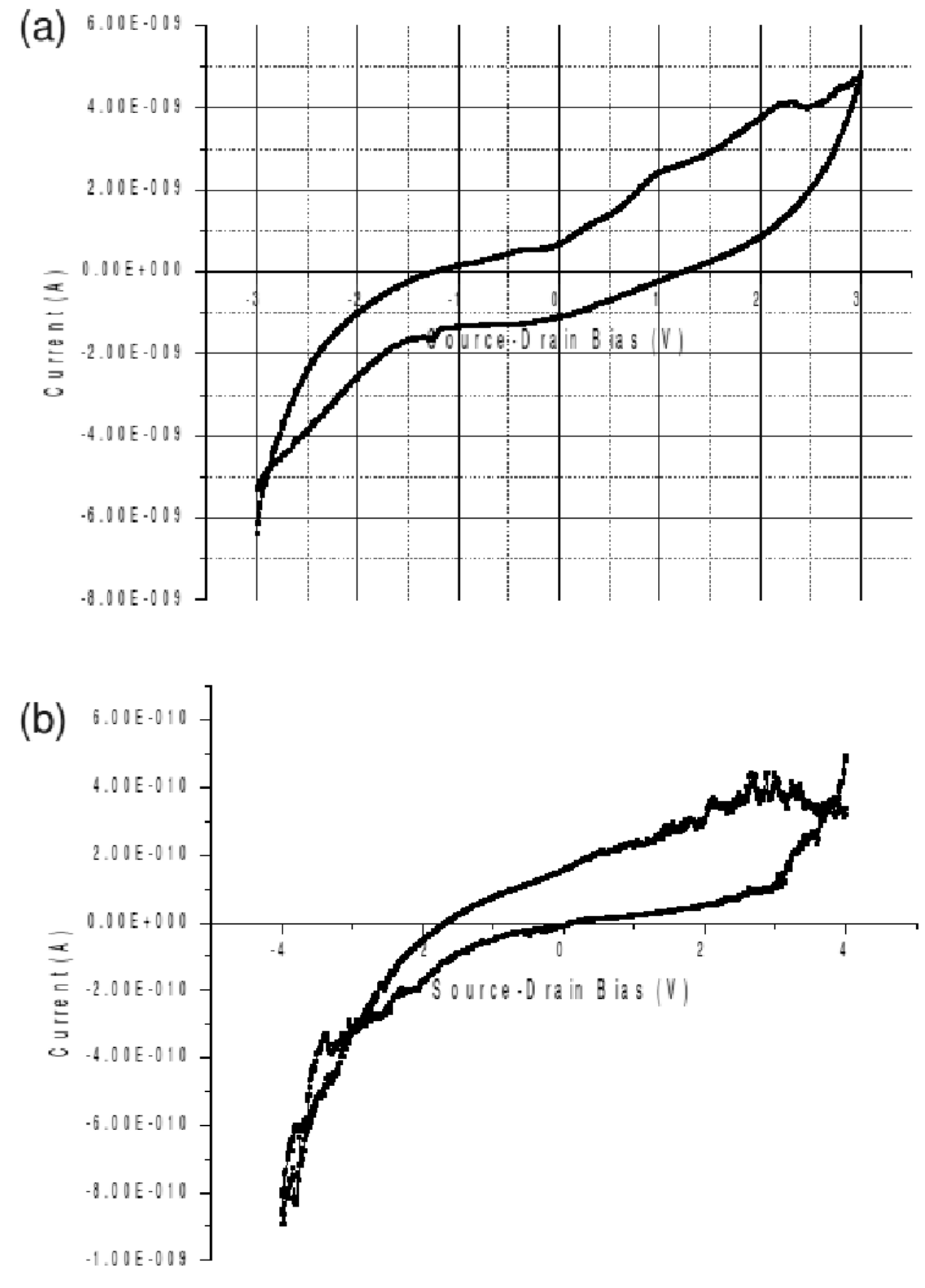

Figure 7.6: Representative I-V of incomplete, open initial junctions. Open junctions showed hysteresis with a magnitude dependent on the width of the gap. (a) Hysteresis of a $2 \mu \mathrm{m}$ gap, (b) hysteresis of a $3 \mu \mathrm{m}$ gap. 


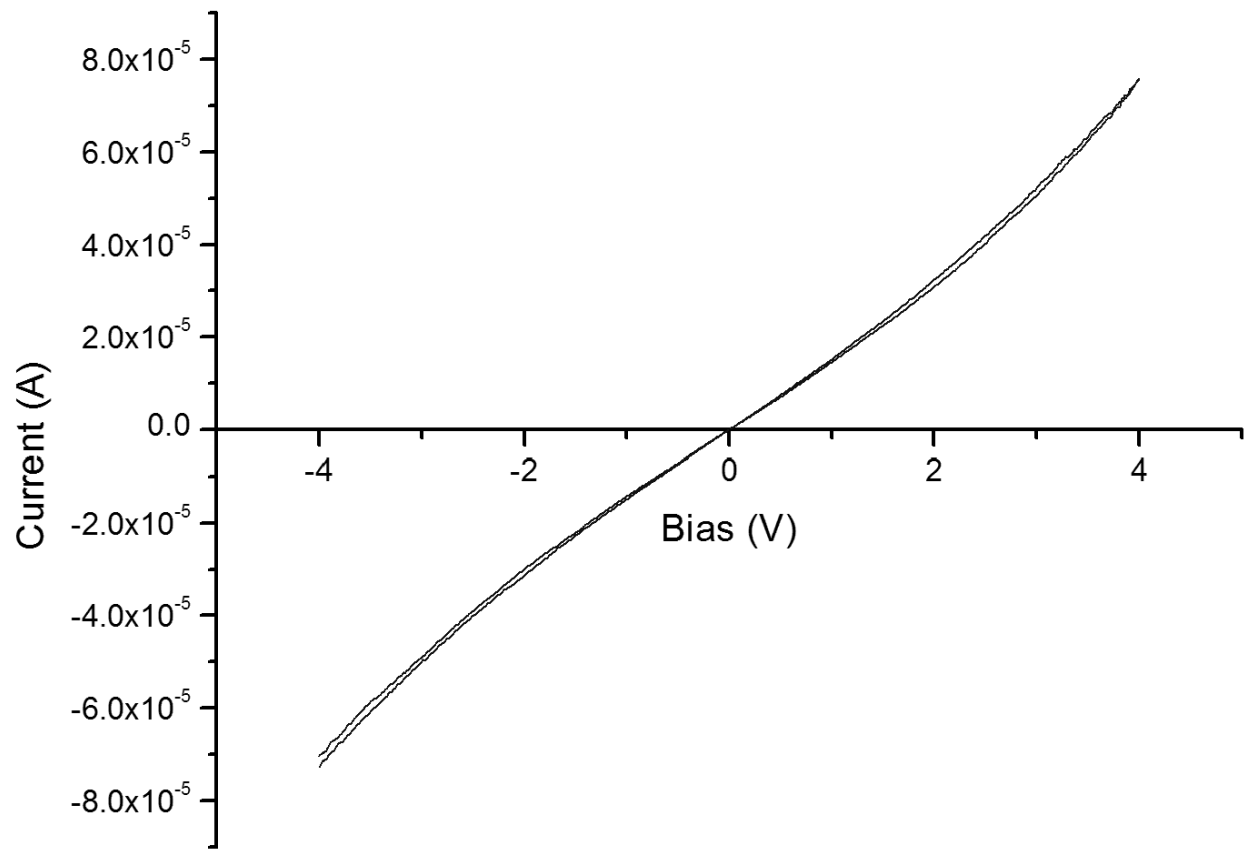

Figure 7.7: I-V of a potential ODT junction on the DEP pattern. Like the other devices in which MWCNTs span the gap, this one shows shorted character. 
for the observed hysteresis. Second, the device junctions are faulty, because every complete junction is indistinguishable from merely laying MWCNTs across gold electrodes. Now the goal became addressing whatever faults in the fabrication we could in order to raise our working device yield.

\subsection{Device Fabrication Improvements}

It is not surprising to have a low yield of working junctions. Heath et al. had $25 \%$ of their junctions stable enough to be usable [9], while Song et al. reported only about $8 \%$ of their break junctions behaving as desired [166]. Fabrication times are long and only one student was assigned to this project; therefore, in order to see results our fabrication process would have to be optimized.

The identified major areas of improvement were:

1. The film may be too rough for a complete, well-formed monolayer.

2. Lift-off leaves very sharp edges which will be difficult to form a complete monolayer on.

3. MWCNT may be shorting to the Cr layer.

The sharp and rough edges left by the lift-off procedure are shown in Figure 7.8. In order to address this issue, a different photolithographic approach would be taken, using etching instead of lift-off. This will result in much smoother edges. The etching process can be used to address the possibility of the MWCNTs shorting to the Cr layer, as well. In this case, we can slightly over-etch the chromium, so it will not extend to the edges of the electrodes, preventing contact. The goal was now to make the flattest films possible combined with photolithographic etching to raise our chances of working junctions.

\subsubsection{Comparison of Deposition Methods}

To begin with, we had to find the deposition method that gave us the initially flattest films. Therefore we investigated three methods and a variety of pre-cleans. For the following analysis, a Veeco Dimension 3000 AFM was used in tapping mode, with an amplitude setpoint of $80 \%$ the free value. Each scan was repeated at 5 different locations for scan sizes of $5 \times 5$ and $1 \times 1 \mu \mathrm{m}$; roughness analysis of a $0.5 \times 0.5 \mu \mathrm{m}$ regions was done using the $1 \times 1 \mu \mathrm{m}$ scans. The uncertainties are simply 


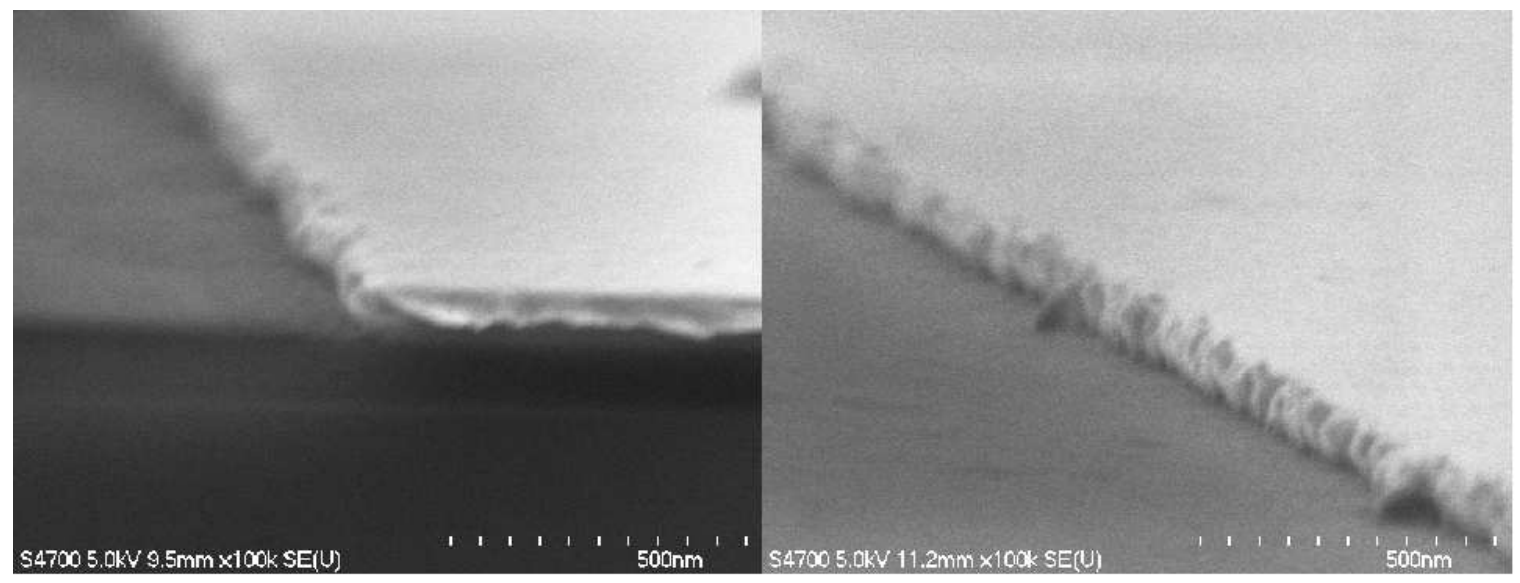

Figure 7.8: SEM of $60 \mathrm{~nm}$ thick Au electrodes, highlighting the sharp and rough edges produced by the lift-off process.

the average deviation from the mean. Two of the measurements, the $250 \mathrm{~nm}$ e-beam and $40 \mathrm{~nm}$ $\mathrm{Cr} / 300 \mathrm{~nm} \mathrm{Au}$, were only measured once at each scan size. The raw average RMS roughness measurements and uncertainties are tabulated in Table 7.5.1. "AI" means the substrate was cleaned before deposition by ultrasonic bath in acetone, then isopropyl alcohol. "PlC" means the substrate was cleaned by plasma before deposition. "PrC" means the substrate was cleaned by a piranha clean (a mix of sulfuric acid $\left(\mathrm{H}_{2} \mathrm{SO}_{4}\right)$ and hydrogen peroxide $\left(\mathrm{H}_{2} \mathrm{O}_{2}\right)$ ). The percentage refers to a difference in sputtering deposition method used by the researchers, "0\%" means the plasma is deignited before the substrate is moved into position under the target, and "10\%" means the plasma power is reduced to $10 \%$ as the substrate is moved into position.

Initially we believed thicker films would be flatter then thinner films given previous PLD experience, but this was not the case. PLD had large particles all over the surface and was ruled out. Plasma sputtering was performed with a variety of pre-cleaning methods of which the plasma clean was the best, though not far ahead of simple acetone/alcohol. The percentage of plasma power at which the substrate is moved beneath the target was a hidden variable. These films were deposited as I was training on the sputter coater, and different people have slightly different methods. I was suspicious that the acetone/alcohol clean was so much worse than the other cleans and, upon investigation, verified it was the technique that was responsible. Roughness was worse for deposition that moved the substrates beneath the target while the plasma was already running at low power, 
Table 7.2: RMS roughness comparison of metal film deposition methods; all numbers are in nanometers. The numbers are the raw averages \pm the average deviation from the mean. AI: substrate was ultrasonicated in acetone, then isopropyl alcohol. PrC: substrate was piranha cleaned. PlC: substrate was plasma cleaned. The percentage refers to what percent of deposition power the sputtering system was at when the substrates were moved beneath the target. The PLD method had large particles all over the surface and was undesirable.

\begin{tabular}{llll} 
& \multicolumn{3}{c}{ Scan Size } \\
Deposition Method & $5 \times 5 \mu \mathrm{m}$ & $1 \times 1 \mu \mathrm{m}$ & $0.5 \times 0.5 \mu \mathrm{m}$ \\
\hline \hline $250 \mathrm{Cr} / \mathrm{Au}$ e-beam (AI) & 2.7 & 1.7 & 1.6 \\
40/300 Cr/Au Sputter (AI) & 2.5 & 1.9 & 1.6 \\
20/40 Cr/Au Sputter (10\%, AI) & $1.7544 \pm 0.0282$ & $1.4072 \pm 0.0854$ & $1.4230 \pm 0.1240$ \\
20/40 Cr/Au Sputter (0\%, AI) & $0.7068 \pm 0.0998$ & $0.7116 \pm 0.1549$ & $0.7164 \pm 0.1495$ \\
20/40 Cr/Au Sputter (0\%, PrC) & $0.8909 \pm 0.1438$ & $0.7834 \pm 0.1127$ & $0.7090 \pm 0.1104$ \\
$20 / 60 \mathrm{Cr} / \mathrm{Au}$ Sputter (0\%, PlC) & $0.7004 \pm 0.0211$ & $0.6642 \pm 0.0226$ & $0.6364 \pm 0.0287$ \\
20/40 Cr/Au PLD (AI) & - & - & -
\end{tabular}

and was much smoother when the plasma is completely off as the substrates move. The acetone/isopropyl was repeated, now matching the other runs, and the results put it about even with piranha clean.

From these results, thinner films deposited by sputter-coating were chosen to test under annealing to see if we could improve the flatness further. The thinner films are important because we want to make sure during dielectrophoresis that the MWCNT lays atop the gold layer and isn't trapped in the gap. E-beam of thin films was not performed because the system is not well characterized (the deposition rates are not well known), the e-beam system is about to be replaced, and the technique was about the same flatness as sputtering.

\subsection{2 $\mathrm{Cr} / \mathrm{Au}$ Annealing}

The next phase was to anneal the substrates under various conditions to try and find a way to flatten the films. The goal was to get the RMS roughness to $5 \AA$ or below, which is the same order as our PPF films, twice the RMS of highly organized pyrolytic graphite, and well within the $1 \mathrm{~nm}$ tolerance required by the integrated circuit industry for defect free submicron features [154]. After a literature search, Reference [168] was found to be a perfect starting point, similar to our conditions 
Table 7.3: RMS roughness comparison of metal film deposition methods; all numbers are in nanometers. The values are the raw averages \pm the average deviation from the mean. The $500{ }^{\circ} \mathrm{C}$ anneals are not shown because they always resulted in highly damaged films.

\begin{tabular}{llll} 
Annealing & $5 \times 5 \mu \mathrm{m}$ & $1 \times 1 \mu \mathrm{m}$ & $0.5 \times 0.5 \mu \mathrm{m}$ \\
\hline \hline None & $0.8908 \pm 0.1438$ & $1.70 .7834 \pm 0.1127$ & $0.7090 \pm 0.1104$ \\
$200{ }^{\circ} \mathrm{C}, 1 \mathrm{hr}$ & $0.7394 \pm 0.5350$ & $0.6340 \pm 0.0140$ & $0.6360 \pm 0.0260$ \\
$200{ }^{\circ} \mathrm{C}, 3 \mathrm{hr}$ & $0.6219 \pm 0.0099$ & $0.5828 \pm 0.0159$ & $0.5848 \pm 0.0255$ \\
$200{ }^{\circ} \mathrm{C}, 5 \mathrm{hr}$ & $0.8020 \pm 0.0180$ & $0.7610 \pm 0.0244$ & $0.7662 \pm 0.0241$ \\
$300{ }^{\circ} \mathrm{C}, 1 \mathrm{hr}$ & $0.6118 \pm 0.0715$ & $0.5332 \pm 0.0337$ & $0.5394 \pm 0.0275$ \\
$300{ }^{\circ} \mathrm{C}, 3 \mathrm{hr}$ & $0.5812 \pm 0.1619$ & $0.4272 \pm 0.0139$ & $0.4324 \pm 0.0129$ \\
$300{ }^{\circ} \mathrm{C}, 5 \mathrm{hr}$ & $0.6222 \pm 0.0174$ & $0.5940 \pm 0.0300$ & $0.5974 \pm 0.0387$
\end{tabular}

and goals. According to their analysis, surface diffusion was the main mechanism for film changes under vacuum annealing at $200{ }^{\circ} \mathrm{C}$ and below, while above $200{ }^{\circ} \mathrm{C}$ other mechanisms dominate. With this in mind, times of 1,3 and 5 hours were investigated, and temperatures of 200, 300 and $500{ }^{\circ} \mathrm{C}$. Substrates were sputter coated to a thickness of $20 \mathrm{~nm} \mathrm{Cr}$ and $40 \mathrm{~nm}$ Au. Annealing took place in a quartz tube furnace evacuated to a pressure of $20 \mathrm{mTorr}$. After the full annealing time, the heater was turned off and the system allowed to cool to $200{ }^{\circ} \mathrm{C}$ before opening the top of the heater and allowing the system to cool more quickly; this was done to prevent thermal shocking of the thin film. The roughness analysis was done using the same procedure as the deposition comparison, and the results are summarized in Table 7.5.2. All films at $500{ }^{\circ} \mathrm{C}$ were severely damaged and unusable. It is pretty clear that the flattest films with the least variance were made at $300{ }^{\circ} \mathrm{C}$ and 3 hours.

\subsection{Additional Problems and Film Improvements}

Now that we had everything in place, the first fabrication was begun. The envisioned design process is shown in Figure 7.9. In this new process, the plasma sputter deposited 20/40 nm $\mathrm{Cr} / \mathrm{Au}$ films are deposited and annealed first. Photoresist is then patterned atop the film, covering the electrode regions and not the gap regions. A two-step etch process takes place to remove the $\mathrm{Au}$ and then the $\mathrm{Cr}$ from the gaps, leaving smooth edges on the electrodes. The remaining protective layer of 
(a)

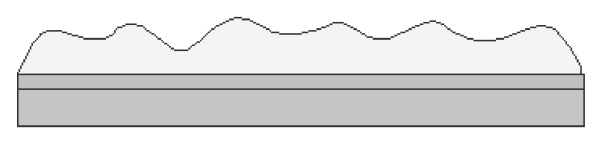

(b)
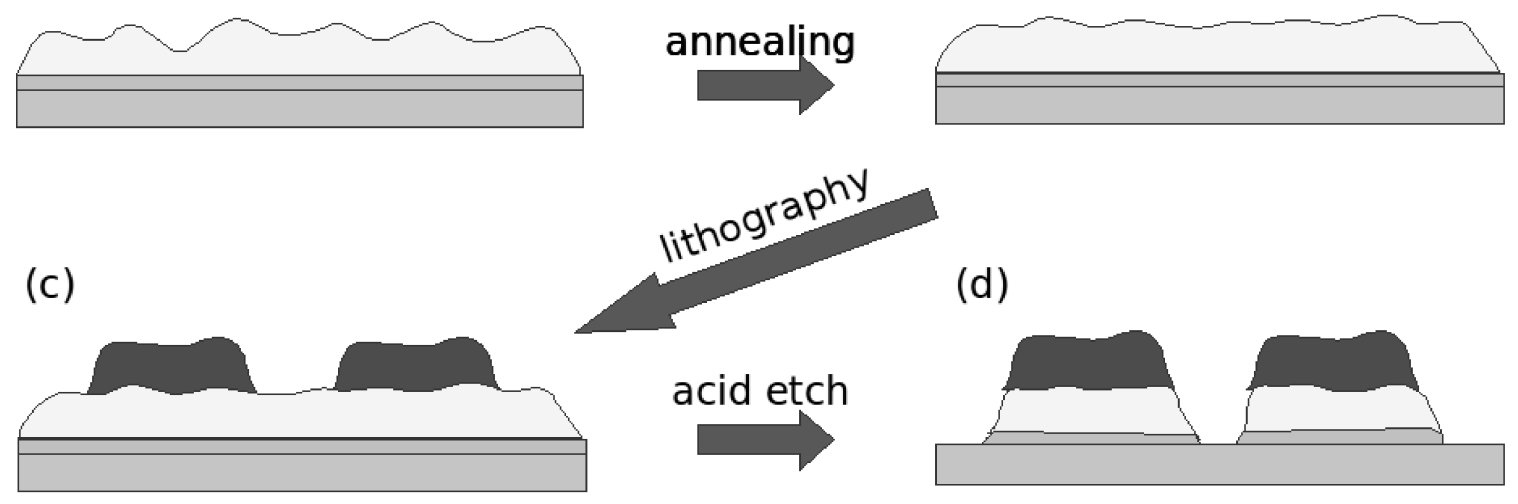

(d)

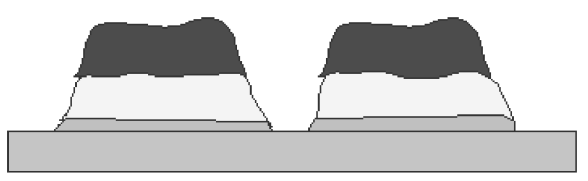

(f)

(e)
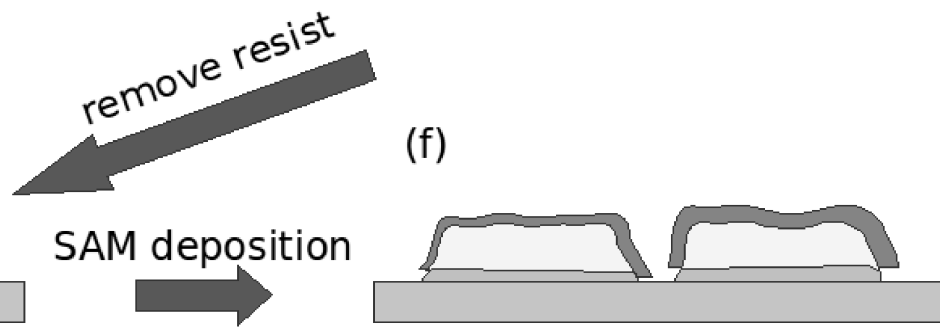

Figure 7.9: The envisioned design process for the annealed, etched devices. A plasma sputter deposited $20 \mathrm{~nm} \mathrm{Cr} / 40 \mathrm{~nm}$ Au film, (a), is thermally anneal it to make a flatter film, (b). Photoresist is patterned atop the film leaving the electrodes regions covered and the gap regions exposed, (c). Etching takes place, leaving the metal film in the same pattern as the resist, (d). The resist is removed, revealing the final electrode pattern, (e), and then the SAM monolayer is deposited, (f).

photoresist is removed and, after cleaning, the ODT is deposited.

However, we found we could not do this method - the annealed films simply do not etch. Based on the rate of Au etchant, the Au of our thickness should be removed in roughly 20 seconds. However, the annealed films didn't etch even over 3 minutes of immersion, Figure 7.10(b). It was clear by eye that the reaction that had been taking place was not at all uniform, and under optical microscope it was shown that most gold was in place, but pitting to the substrate occurred in random locations, Figure 7.10(c). I suspected the annealing was the reason the films were not etching and had another sample whose films were deposited during the same sputter but which had not yet been annealed; this sample etched perfectly Figure 7.10(a).

The quartz tube furnace used for annealing also hosts a variety of other experiments and the idea that some sort of contamination was causing the etching problem needed to be tested. A $60 \mathrm{~nm} \mathrm{Au}$ 


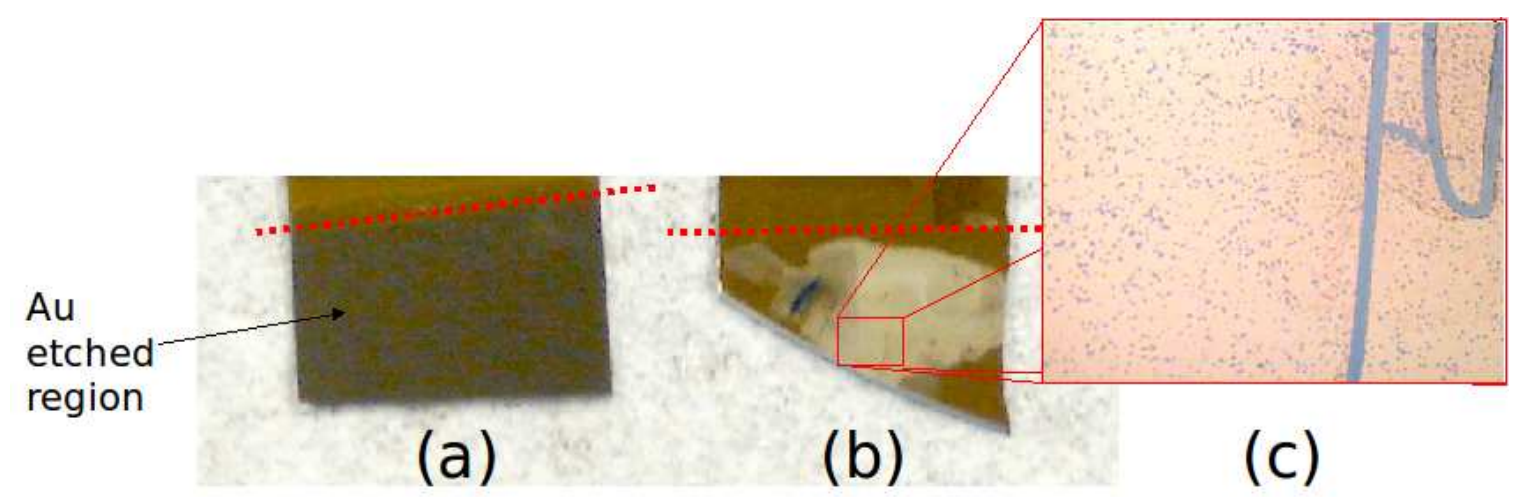

Figure 7.10: Comparison of $\mathrm{Au}$ etching on unannealed and annealed $\mathrm{Cr} / \mathrm{Au}$ films. The dotted line represents the line beneath which the film was submerged in etchant. (a) Before annealing, the $\mathrm{Au}$ film etches away cleanly, at the expected rate $(\sim 20 \mathrm{~s}$ for $40 \mathrm{~nm})$. (b) After annealing, the film would not etch after up to 3 minutes, and was completely non-uniform. (c) A close-up of the region in the etchant shows pitting down to the substrate (the scratch is present to show the color in the pits is the same as the color of the substrate).

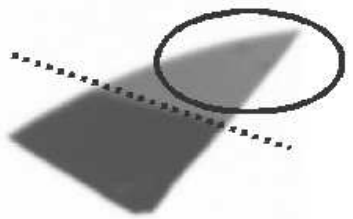

(a)

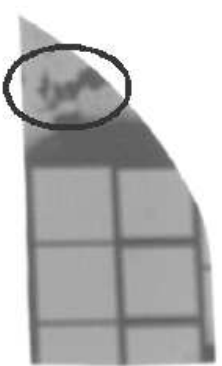

(b)

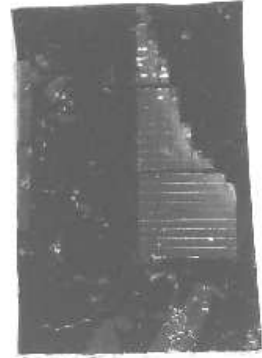

(c)

Figure 7.11: Comparison of different Au films. (a) Annealed pure Au films etch well, and shown extremely good adhesion during a "tape test," (circled region shows almost no Au removed after four applications). (b) Unannealed $\mathrm{Cr} / \mathrm{Au}$ films show poorer adhesion than the annealed $\mathrm{Au}$, with a single application of tape removing a large portion of the film (circled region). (c) Pure gold films must be annealed before etching, or the patterns are removed from the surface of the substrate during the etch. 
Table 7.4: RMS roughness comparison of unannealed and annealed $60 \mathrm{~nm}$ Au films; all numbers are in nanometers. The values are the raw averages \pm the average deviation from the mean.

\begin{tabular}{llll} 
& \multicolumn{3}{c}{ Scan Size } \\
Annealing & $5 \times 5 \mu \mathrm{m}$ & $1 \times 1 \mu \mathrm{m}$ & $0.5 \times 0.5 \mu \mathrm{m}$ \\
\hline \hline None & $0.2800 \pm 0.0360$ & $0.2810 \pm 0.0213$ & $0.2813 \pm 0.0122$ \\
$300{ }^{\circ} \mathrm{C}, 3 \mathrm{hr}$ & $0.5758 \pm 0.0370$ & $0.4524 \pm 0.0345$ & $0.4040 \pm 0.0628$
\end{tabular}

film with no $\mathrm{Cr}$ was deposited and annealed under identical conditions, and this film etched perfectly, Figure 7.11(a). When handling the sample, I was impressed with how good the Au adhesion seemed to be even without the chromium. I subjected the film to a "tape test" where a piece of Scotch tape is pressed firmly to the film surface and is then removed. The annealed pure gold film was almost completely intact after four applications of the tape; the region tested is indicated by the circle in 7.11(a). By comparison, a large portion of the film was removed from the $\mathrm{Cr} / \mathrm{Au}$ films after just a single application of tape, circle in 7.11(b).

This was certainly a fortuitous discovery! The annealed pure gold film has better adhesion than the $\mathrm{Cr} / \mathrm{Au}$ film, has no chromium at all, so there is no potential for shorting to the chromium and fabrication has fewer steps. I intended to do a comparison of pure gold films and whether it was better to anneal first and then pattern by etching, or pattern first and then anneal; however, without annealing, the Au film is removed during the etching process, as shown by an only partially etched film in Figure 7.11(c). The comparison of $60 \mathrm{~nm} \mathrm{Au} \mathrm{films} \mathrm{without} \mathrm{and} \mathrm{with} \mathrm{annealing} \mathrm{are} \mathrm{shown} \mathrm{in}$ Table 7.6. The annealed pure gold films fall within our RMS roughness requirement, so they will be the basis for the devices to follow. Interestingly, the pure gold films that have not been annealed are the flattest I characterized, and the annealing process makes the film rougher. This could mean that the chromium was responsible for the more extreme roughness for previous deposition.

\subsection{Device Testing}

The full process used for device construction is now:

1. Clean substrates with immersion in an ultrasonicating bath of acetone, then ethanol, followed rinsing in a stream of ethanol and drying by $\mathrm{N}_{2}$. 
2. $60 \mathrm{~nm}$ Au films deposited by plasma sputtering.

3. Substrates are annealed for $3 \mathrm{hrs}$ at $300{ }^{\circ} \mathrm{C}$ in vacuum.

4. Photolithography is used to pattern a photoresist atop the Au film.

5. The Au is etched, forming into the desired electrode pattern.

6. The remaining resist is removed with acetone followed by ethanol.

7. The substrates are placed in ODT solution for 48 hours in a nitrogen environment glove box for SAM formation.

8. Samples are removed and rinsed thoroughly with ethanol.

9. Dielectrophoresis is performed to make the top MWCNT connections.

10. The devices are tested.

One small drawback at this stage is that the $2 \mu \mathrm{m}$ features are not yet workable with the etching method. Therefore, for these first junctions, simple pairs of square pads are used to make the device. The path of current flow through the device is identical to the original pattern, but the electrodes are much wider, so far more ODT molecules and MWCNTs are involved. The square pads are in two types, one of $100 \times 100 \mu \mathrm{m}$ with a $10 \mu \mathrm{m}$ gap between, and one of $1000 \times 1000 \mu \mathrm{m}$ with a $100 \mu \mathrm{m}$ gap between. Both types of patterns were used just to maximize the amount of tests per substrate. Though the gaps are wider than the intended final devices, it should have no effect on their behaviour, only the magnitude involved. In fact, the larger size means each junction is more likely to have shorts, so if the large device works, the smaller ones should definitely work once the photolithography and etching are optimized.

As in previous experiments, some junctions showed ohmic curves that indicate a short of some type, bypassing the monolayer. Examples are shown in Figures 7.12 and 7.13.

However, two $1000 \times 1000 \mu \mathrm{m}$ patterns, however, showed more interesting behaviour. The first such junction showed that between 2 and -2 volts, there was a repeatable pattern of hysteresis, Figure 7.16. Within this range of $\pm 2 \mathrm{~V}$, the $\mathrm{I}-\mathrm{V}$ characteristics are very stable; according to our STM results, this region is the current gap region. When swept performed a voltage sweep over a 


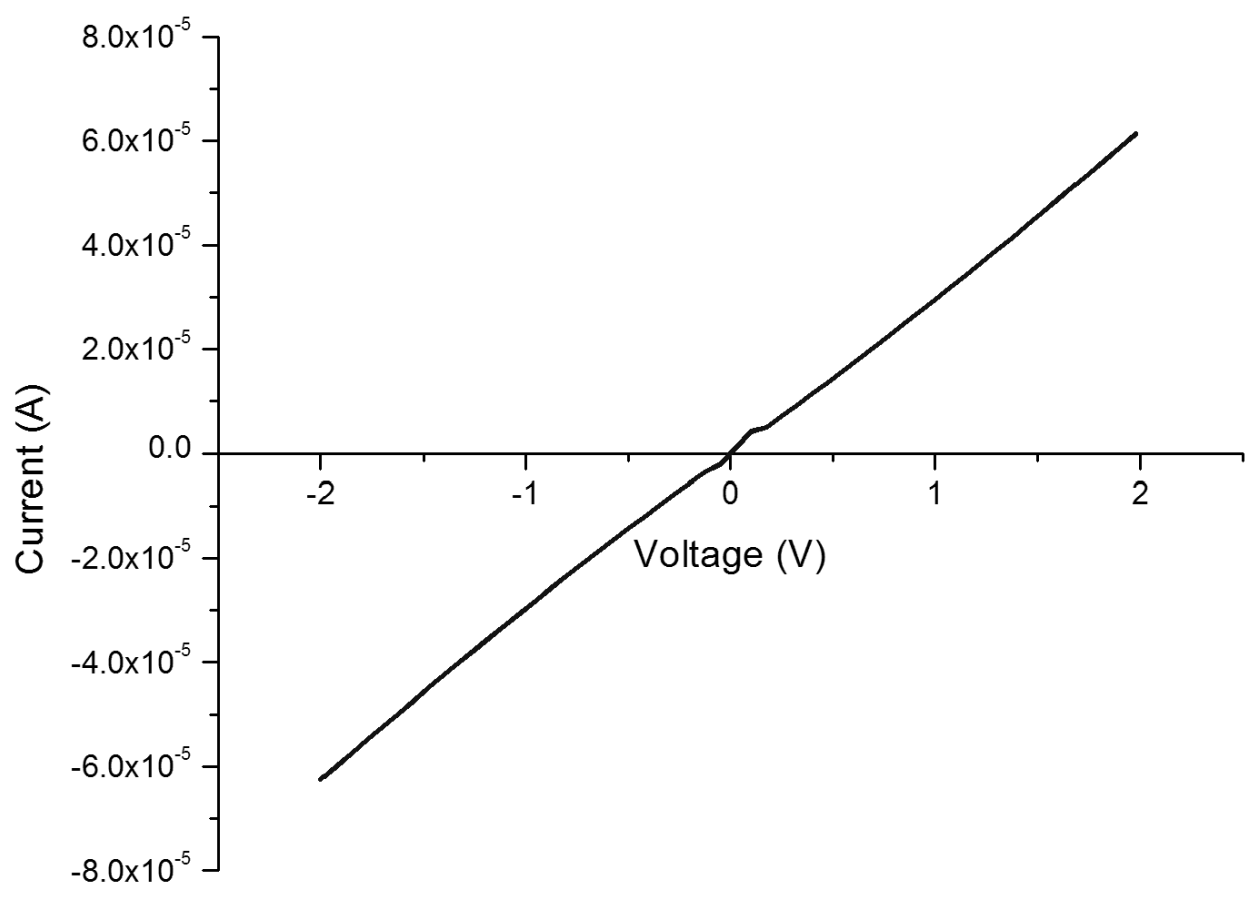

Figure 7.12: $\mathrm{I}-\mathrm{V}$ characteristics of a $100 \times 100 \mu \mathrm{m}$ square pattern with $10 \mu \mathrm{m}$ gap showing shorted behaviour. 


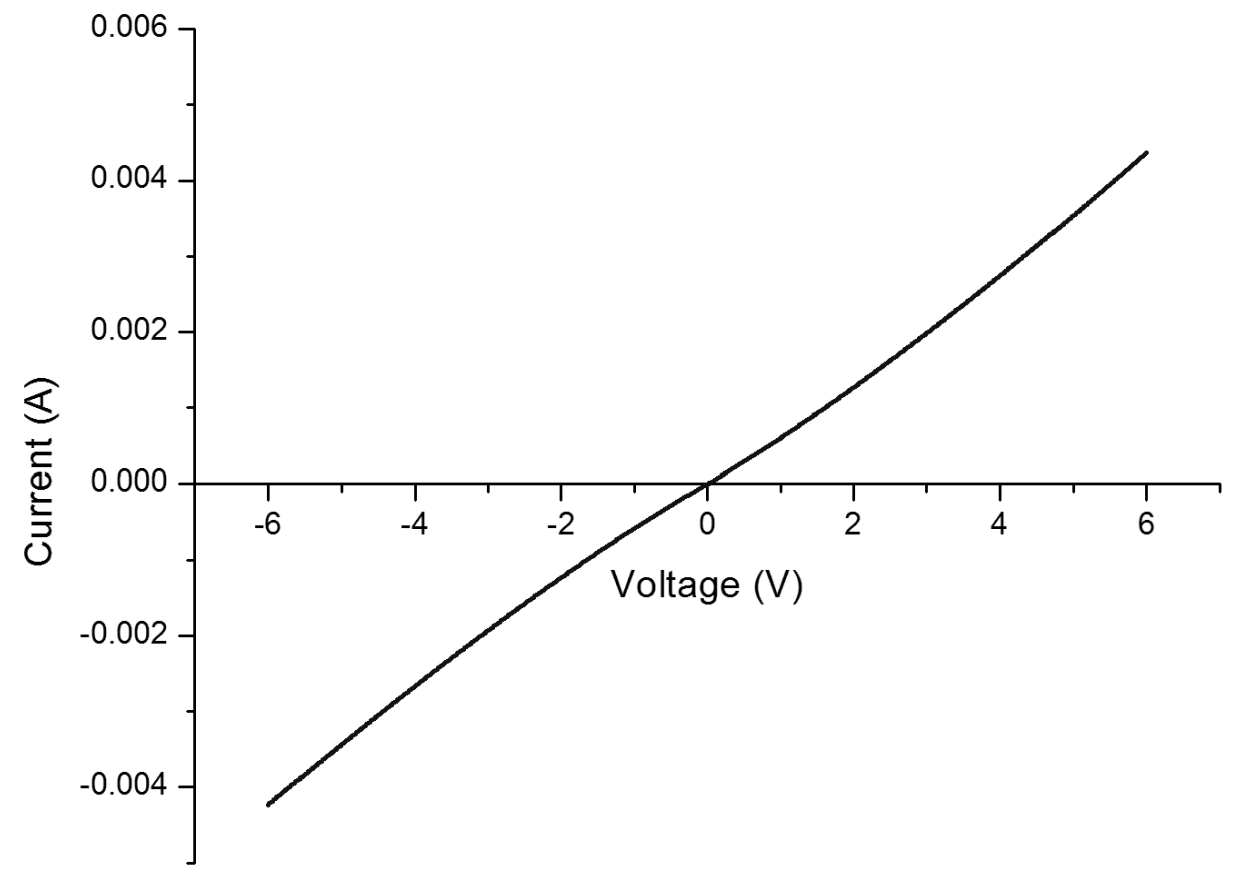

Figure 7.13: I-V characteristics of a $1000 \times 1000 \mu \mathrm{m}$ square pattern with $100 \mu \mathrm{m}$ gap showing shorted behaviour. 


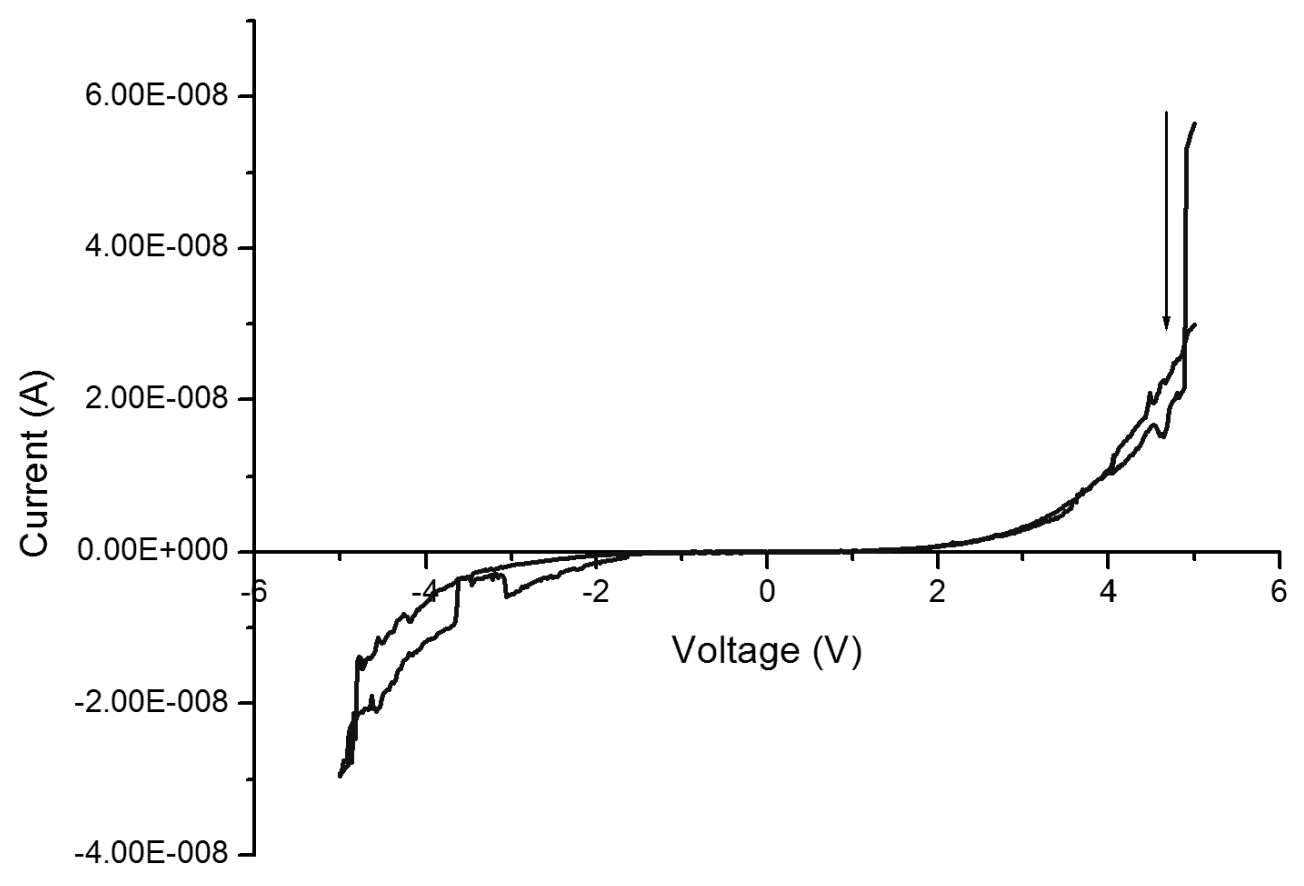

Figure 7.14: I-V characteristics of a $1000 \times 1000 \mu \mathrm{m}$ square pattern with $100 \mu \mathrm{m}$ gap. The observed curve is very much like the ODT measured by STM, but with a hysteresis effect.

larger range, a curve almost identical to the ODT measurement by STM was found, with a wide current gap region of high resistance, Figure 7.14. However, going outside of the current gap region introduced instability and a hysteresis effect. Pushing the voltage to \pm 12 volts irreparably damaged the device (Figure 7.17), and following scans showed only noise (Figure 7.18).

It should be noted that this electrode pair was not one that we performed dielectrophoresis on directly. Only one of the two pads was contacted by probe in the DEP process, and the other was at a floating potential, so MWCNTs deposited in the gap between them but at a lower density. We will see that the next junction shows similar behaviour for the same electrode dimensions, but at a much higher current. That is because MWCNTs were deposited between those two directly, so the density of current carriers should be much higher, leading to a lower resistance. 


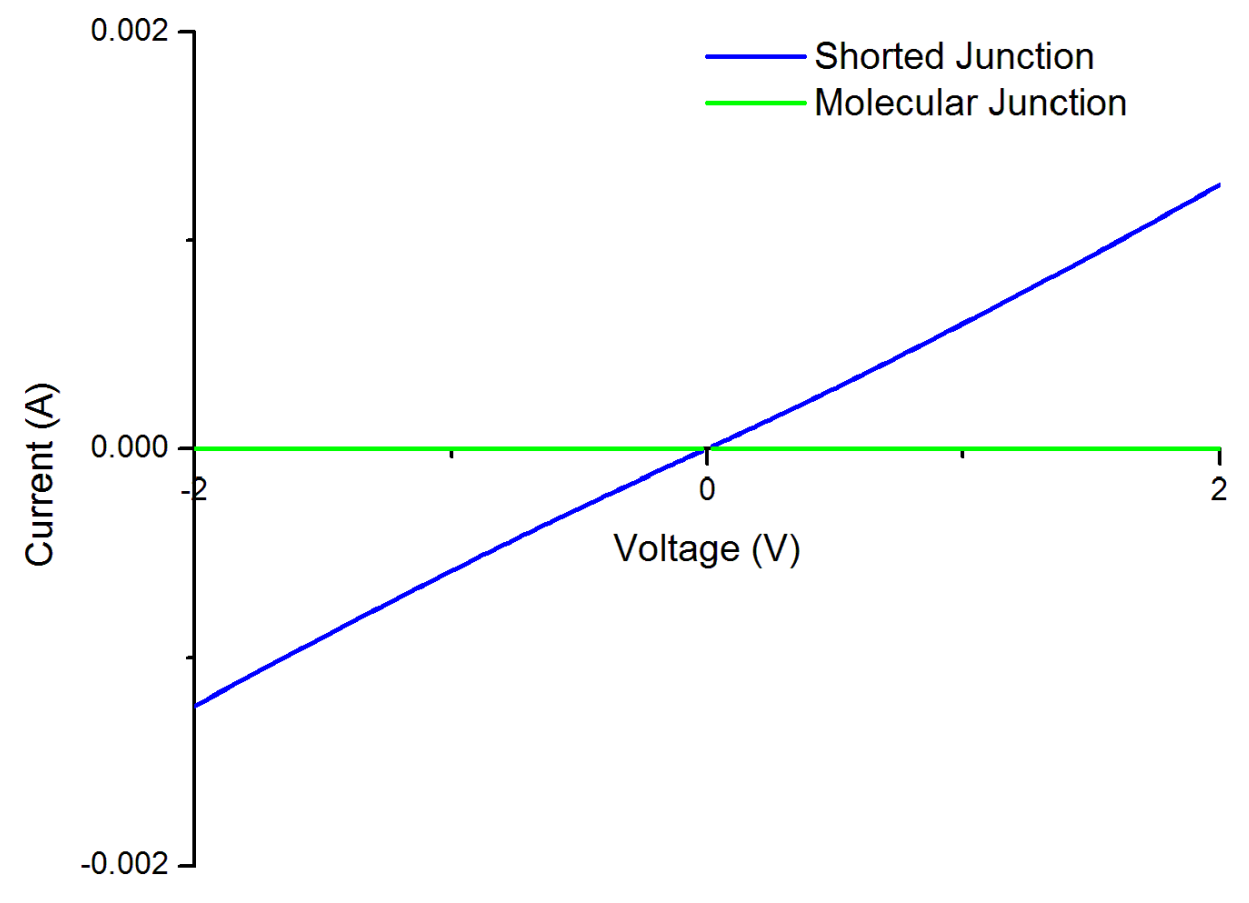

Figure 7.15: Comparison of shorted and working molecular junctions on $1000 \times 1000 \mu \mathrm{m}$ square patterns. 


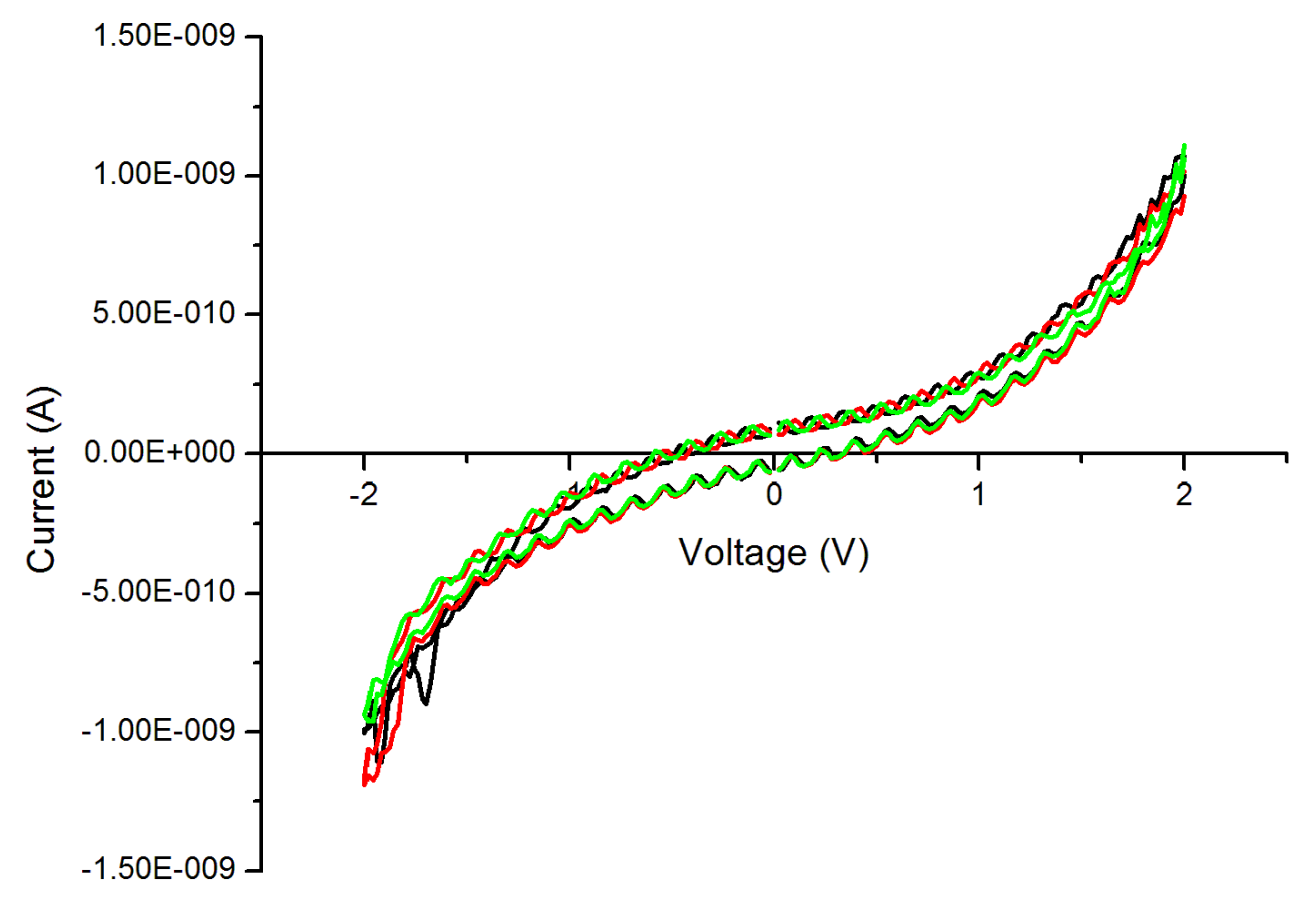

Figure 7.16: I-V characteristics of a $1000 \times 1000 \mu \mathrm{m}$ square pattern with $100 \mu \mathrm{m}$ gap showing hysteresis. 


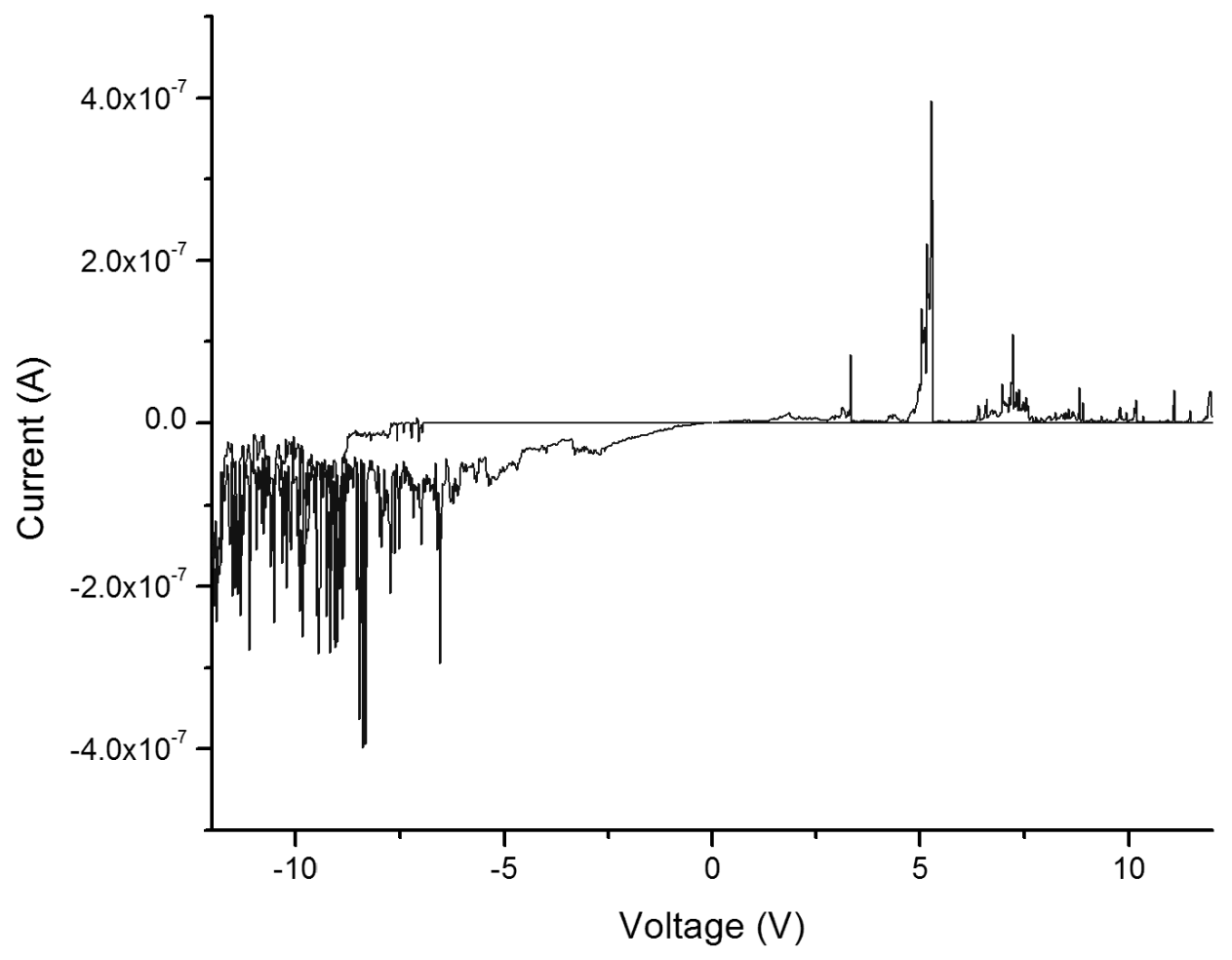

Figure 7.17: I-V characteristics showing Junction 2 being broken by too much applied voltage. 


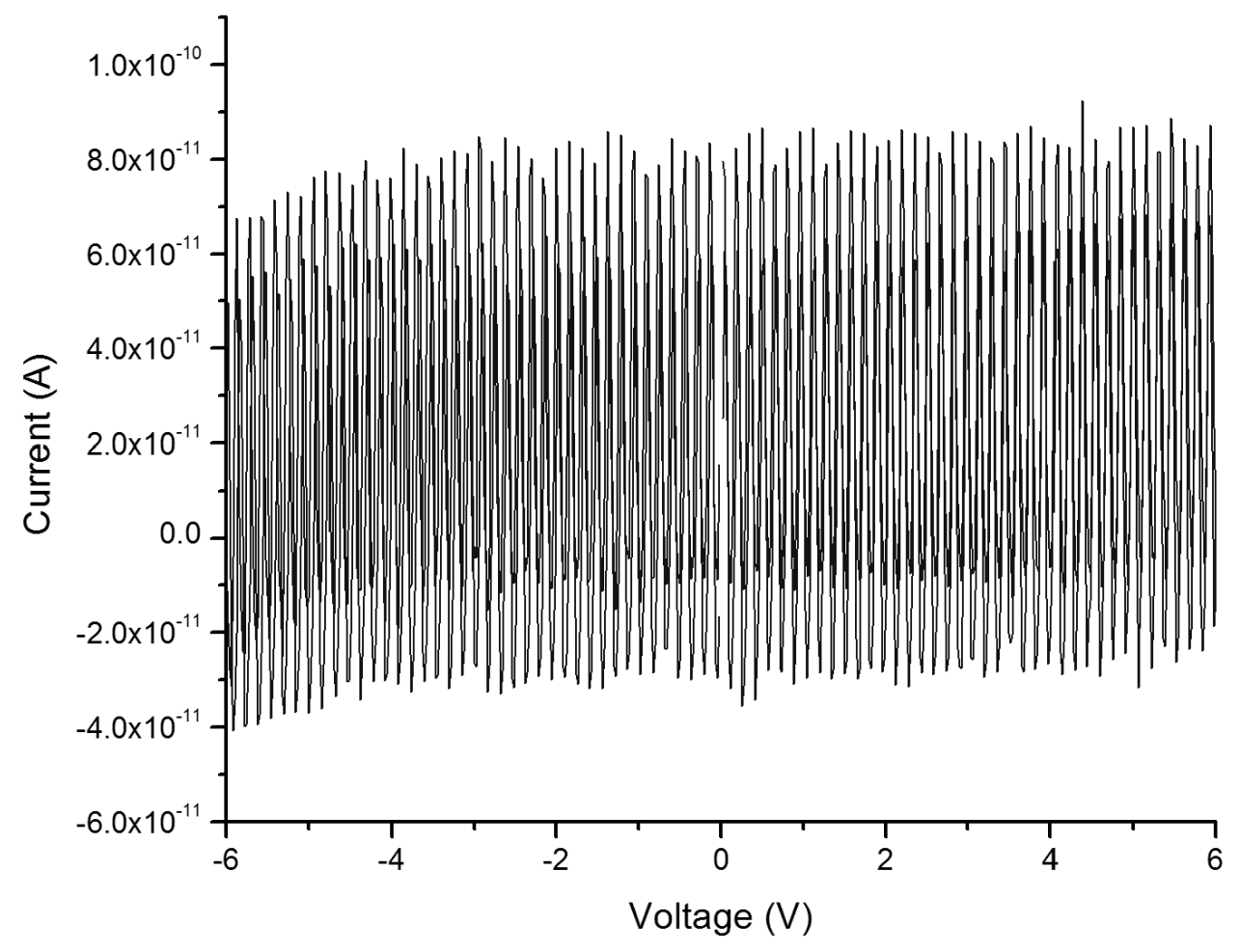

Figure 7.18: I-V characteristics of Junction 2 after breakage shows nothing but noise. 

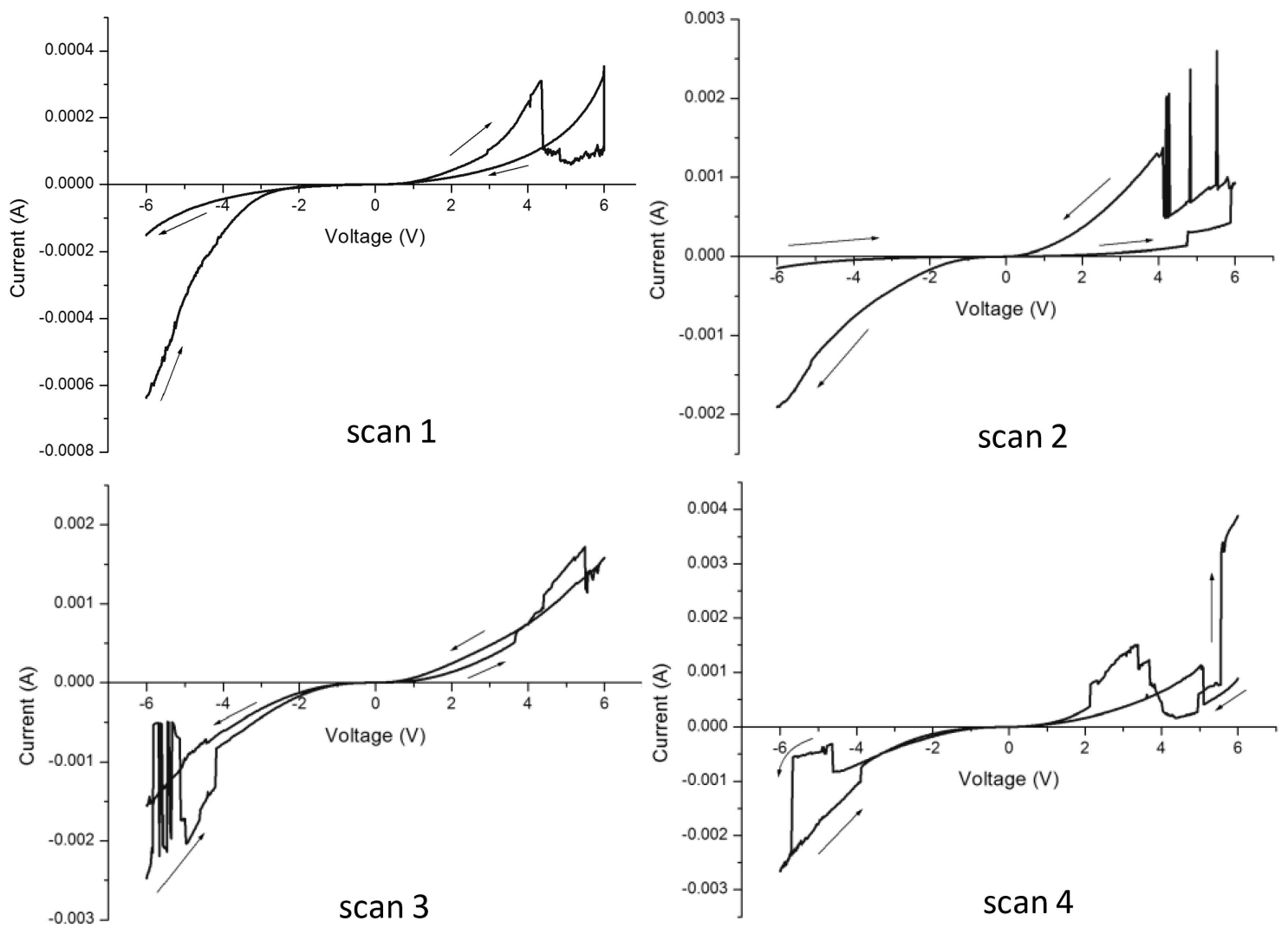

Figure 7.19: I-V of the first 4 scans of the second working junction.

This second junction also exhibited characteristics of ODT in its I-V curves: the current gap region of high resistance expected with ODT. Also like the first junction, outside of the current gap there is instability and hysteresis. Repeated scans were performed on this junction, each within a minute of the next. Figure 7.19 shows the first four scans together, and Figure 7.20 shows the latter three and a comparison of the first and seventh scan. A few things are evident. First, unlike voltage sweeps done only within the current gap region, here we see changes to the character of the curve. In general, the overall resistance of the junction decreases with repeating scans, which is dramatically shown in the comparison of scans 1 and 7 in Figure 7.20 and in Figure 7.21. Second, the current flow is significantly less stable outside of \pm 2 volts. Thirdly, the magnitude of the observed hysteresis has no simple link to the scan number, though after a point it has disappeared. Last, the current gap region shrinks in width with repeated scans. 

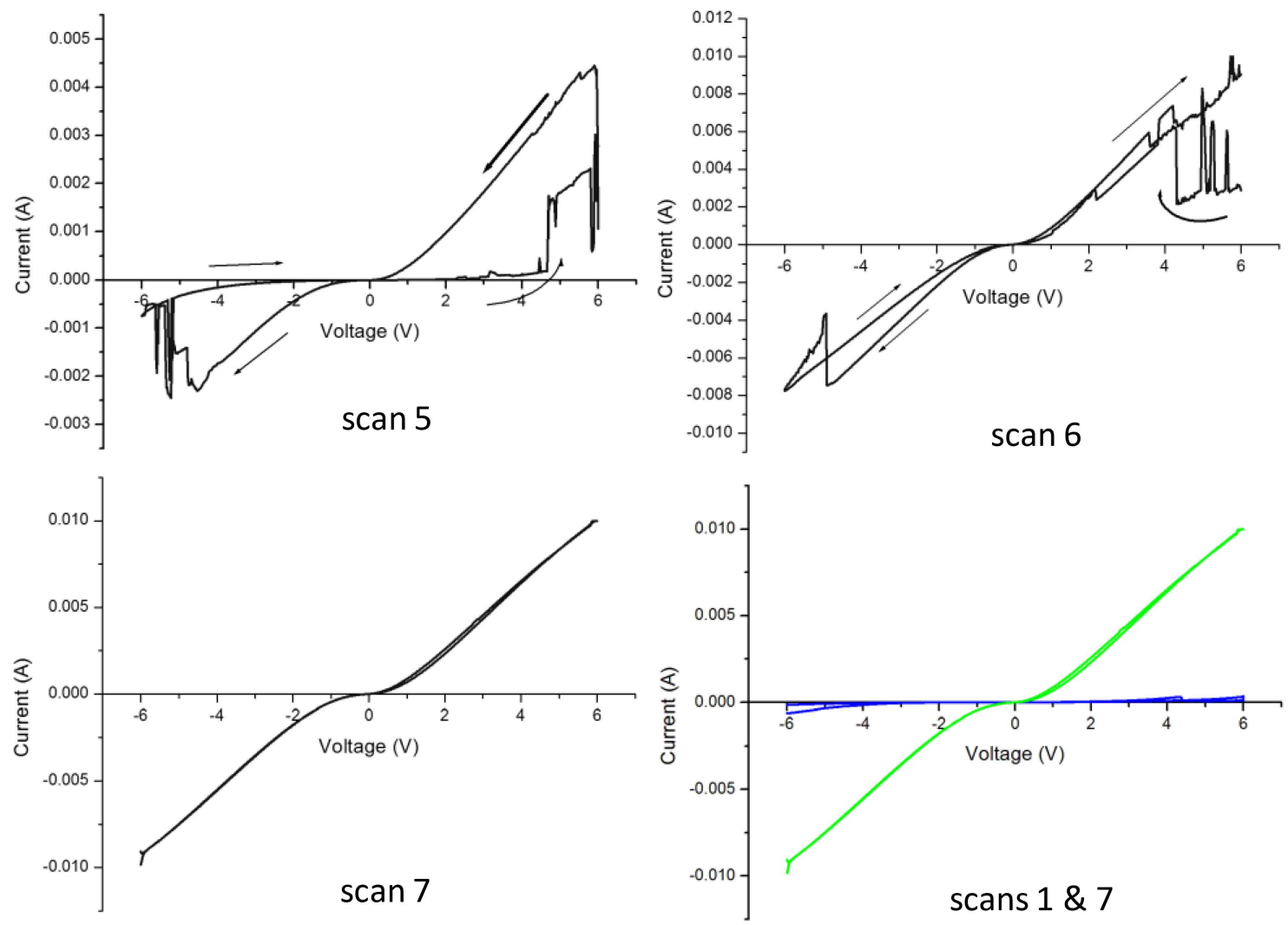

Figure 7.20: I-V of the last three scans of the second working junction, and a comparison of the first and last. 

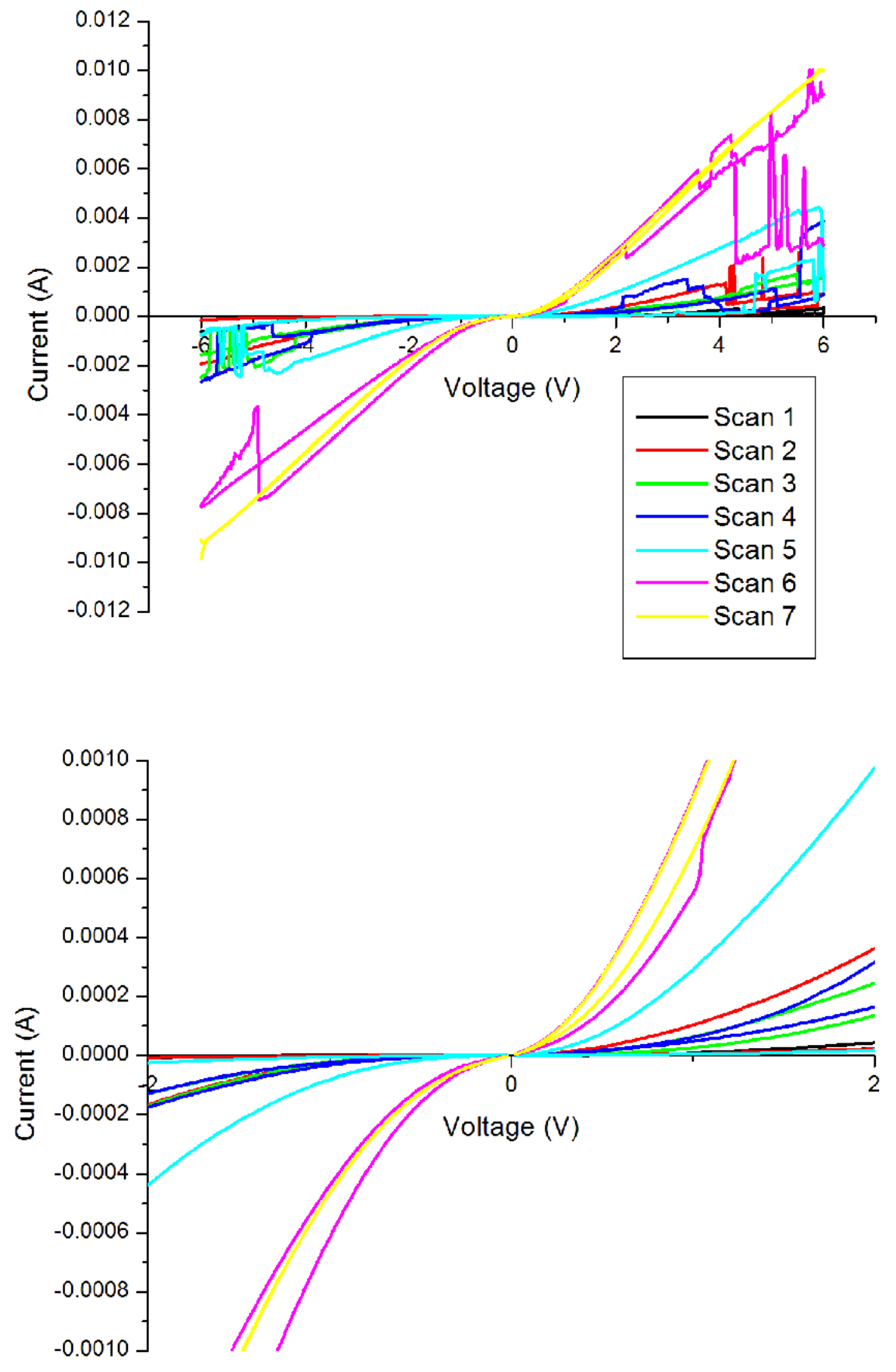

Figure 7.21: I-V of the first 7 scans of Junction 3. 


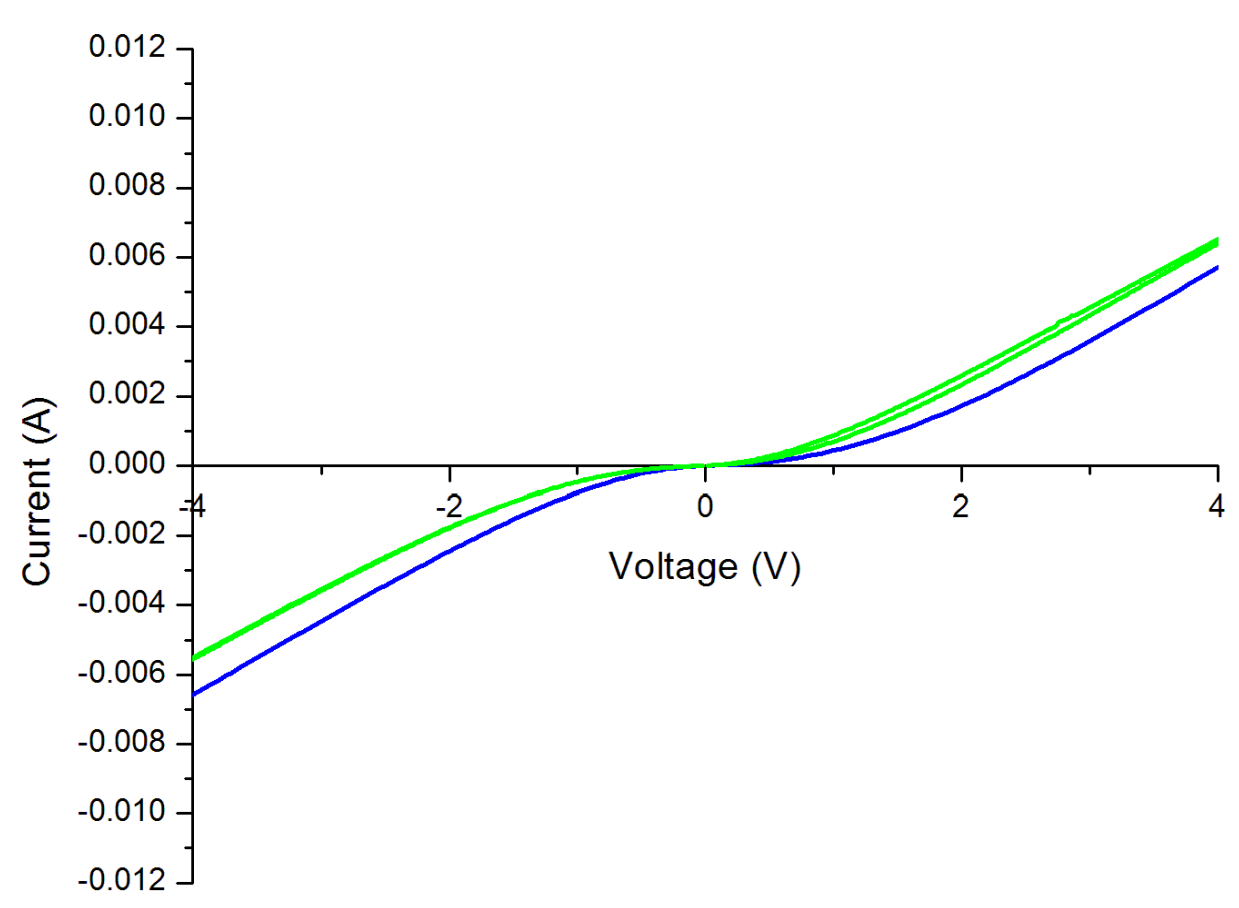

Figure 7.22: I-V of the second molecular junction on the initial date, and two weeks after. The observed changes to the I-V character are permanent.

The hysteresis can be attributed to the poor top contact and the fact that the insulating SAM is likely undergoing breakdown in the region outside the current gap. Breakdown of the SAM could explain the decreasing resistance, and should mean the conductance change is permanent. Revisiting the junction two weeks later shows the high conductance state has not relaxed, and is a permanent change, as shown in Figure 7.22. It appeared to be a stable condition at this point, and showed identical curves under the following scans of \pm 4 V, Figure 7.23.

We were interested in how far we could push the junctions, and we had already seen that $12 \mathrm{~V}$ would destroy them. Therefore, the second junction was tested again at $\pm 8 \mathrm{~V}$ to see the behaviour, Figure 7.24. By moving outside the $\pm 4 \mathrm{~V}$ range instability is reintroduced to the current. When repeating the scan an abrupt drop in current was observed. Initially we thought the junction was 


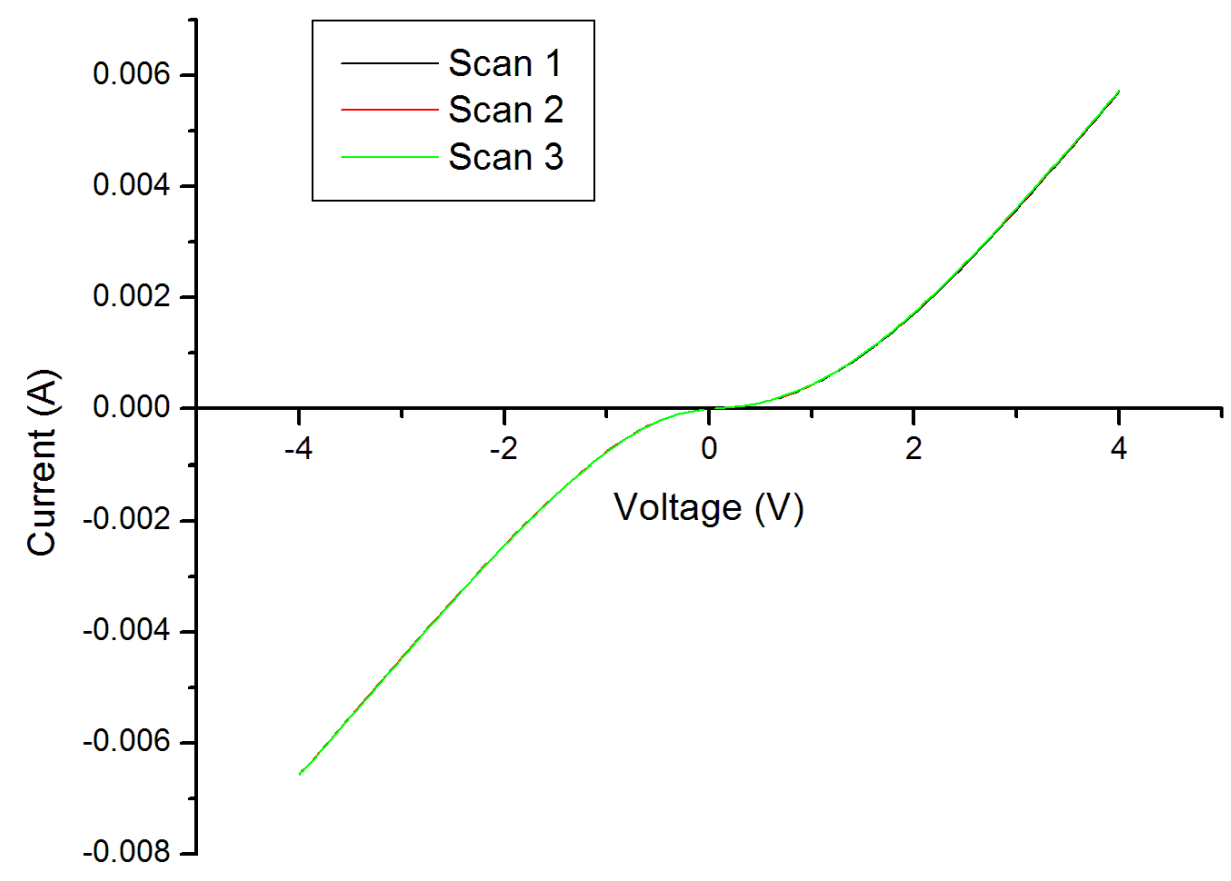

Figure 7.23: Repeated scans at $\pm 4 \mathrm{~V}$ of the second junction, showing identical curves for each scan. This state is stable in this voltage range. 

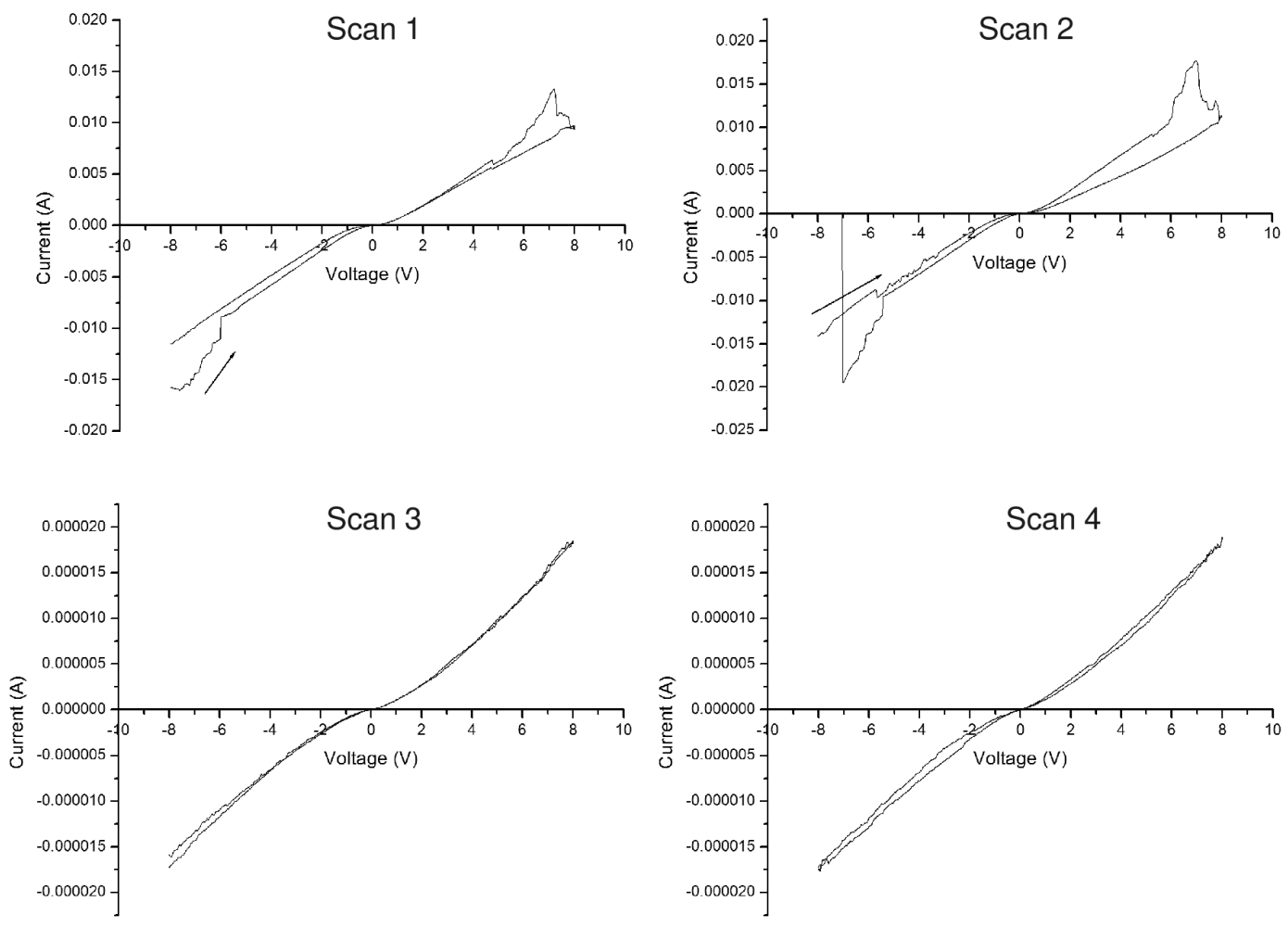

Figure 7.24: Repeated scans at $\pm 8 \mathrm{~V}$ of the second junction. In this extended voltage range, instability is reintroduced to the curves. During the second scan, the current abruptly drops. However, the junction is not open, but only at lower current range, as shown in scans 3 and 4 .

damaged and would show open circuit behaviour, however subsequent scans showed the magnitude of the current had dropped dramatically, but the shape of the scan was as it was before the $\pm 8 \mathrm{~V}$ scans.

\subsection{Summary and Conclusions}

Molecular junctions of the Au-ODT-MWCNT type were attempted. Initial junctions only showed ohmic behaviour, not the expected insulating character of ODT. After a series of experiments designed to improve the yield and address possible fabrication shortcomings (Au film roughness, edge roughness and shorting to the exposed Cr layer), the newly designed junctions were tested. This 
time, two potential junctions showed the expected character of ODT, a current gap region in the $\pm 2 \mathrm{~V}$ range and higher current flow outside of it.

The molecular junctions showed, stable, repeatable scans within the current gap, but hysteresis and current instability occur outside the gap. Further, scanning outside the gap leads to permanent change in the gaps, leading to higher current flow and shrinking of the current gap region. We think this occurs as the ODT layer suffers breakdown and is damaged, leading to a mixed layer of intact and damaged ODT. This is consistent with reports that ODT suffers breakdown around $3 \mathrm{~V}$ [169]. If the voltage scan is limited to $\pm 4 \mathrm{~V}$, it reaches a stable (for at least several scans) higher current state. Based on the work of Beebe and Kushmerick, [170], a likely candidate for the increased conductance in the junction is diffusion of gold molecules into the monolayer. However, the MWCNTs themselves seem to take damage if scanned between $\pm 6 \mathrm{~V}$, probably due to joule heating. It must be the MWCNTs that begin to take damage, because unlike the other observed current changes, this leads to lower current flow. Finally, if pushed to $\pm 12 \mathrm{~V}$, the junction suffers catastrophic damage and becomes open.

These are the observations based on two junctions, and may not be enough to draw firm general conclusions. However, the curves for the two working junctions match the previous reports and our own STM analysis, and the character of the junctions is unlike any other I-V of MWCNTs between electrodes observed during this research. We think the observed instability and breakdown outside the current gap region further support this claim, since breakdown is the expected response. Therefore, we have shown the ability to make molecular junctions which retain the electric characteristics of the chosen SAM. This method can be used to test further junctions of ODT, and to test future SAMs as well. In addition, we have shown that molecular electronic devices can be made using common techniques such as annealing, microphotolithography and dielectrophoresis. 


\section{Appendix A}

\section{Equipment and Techniques}

This appendix contains introductions to techniques that are used throughout the dissertation research. The sections are intended to give a basic understanding of what each technique entails, and how its use pertains to my study.

\section{A.1 Photolithography}

\section{A.1.1 Historical Perspective}

Broadly defined, lithography is a process in which copies of a master pattern are transferred onto another solid surface. The word's origin is in the Greek, coming from litho (stone) and gráphein (to write). The word was initially used to refer to the process devised by Aloys Senefelder in 1796 [171]. He found that if stone was properly treated with chemicals and ink, it could transfer an image carved into it onto paper. The treated stone made areas of the paper ink-receptive (the oilreceptive, water-repellent areas) and the other areas ink-repellent (the oil-repellent, water receptive areas). Therefore, the applied ink would form into the desired pattern [171].

The first photolithography would come some years later, following the work of Nicéphore Niépce in 1822. Niépce had been experimenting with resins in the sunlight, and was able to copy an etched print on oiled paper to a glass plate after several hours of sun exposure. The plate was coated with bitumen (asphalt) dissolved in lavender oil; during this process the shaded areas remain soluble, but 
the exposed regions harden. The difference in solubility allows the softer regions to be washed away with turpentine and lavender oil, leaving the harder regions forming the image [171]. Niépce had discovered a negative resist, and following his work many natural materials were found that were also applied as negative resists [172].

In 1827 an engraver by the name of Lemaitre made an etched copy of an engraving of Cardinal d'Amboise. Done using strong acids and a plate developed by Niépce, this marks the first example of pattern transfer by photolithography and chemical milling [171].

It wasn't until the early 1920's that Kalle and Company, AG in Germany, as well as its American subsidiary Azoplate Corporation, developed the first positive photoresist. The resist was based on novolac resins and quinone diazid photoactive dissolution inhibitors. Originally meant to be used for the fabrication of lithographic plates, it became the basis for microcircuitry and modern photolithography [172].

\section{A.1.2 Background Information}

Photolithography is the most commonly used form of lithography [171]. The purpose is to use a photomask to generate a desired pattern on a photoresist-covered wafer. A simplified example introducing basic concepts of a photolithographic process is given in Figure A.1. In this example, a silicon wafer with an oxidized layer is used as a substrate. A positive photoresist is spun atop the wafer, leaving a uniform film. A photomask is then placed in contact with the wafer and the system is exposed to UV light. Areas of the film exposed to the light become more soluble to the developer solution, while the areas shaded by the pattern on the mask remain less soluble. The wafer

is then placed in developer solution, removing the exposed regions and leaving a negative image of the pattern that is ultimately desired. An iron film is coated over the surface, with the remaining film serving as a stencil. Finally, the remaining resist is removed with a stripper, removing any unwanted metal and leaving the desired pattern in a step that is called lift-off.

The full process involves more steps, for example: 


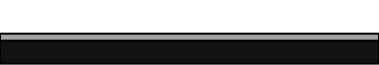

(a) Si wafer with oxide layer serves as the substrate

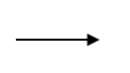

(b) positive photoresist is spin-coated in a uniform layer
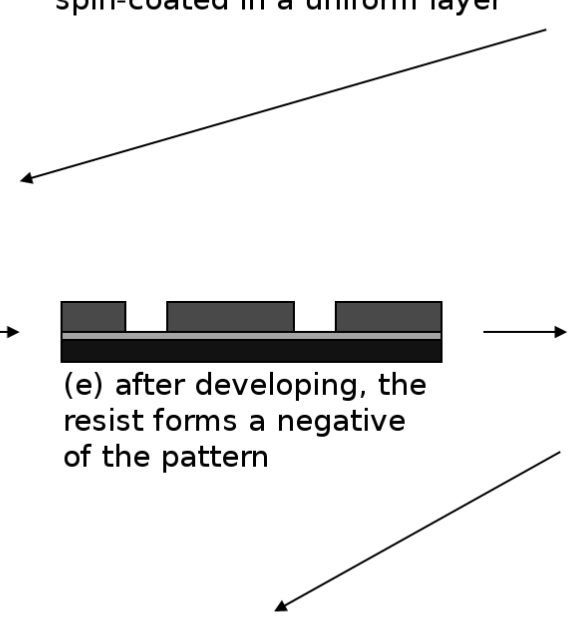

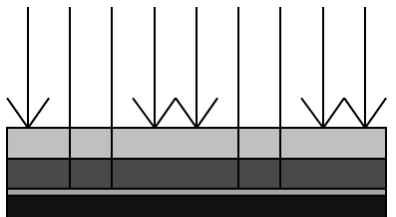

(c) photomask is used to expose only certain areas to the UV (d) the exposed regions are more soluble to the developer

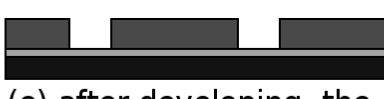

(e) after developing, the resist forms a negative

of the pattern
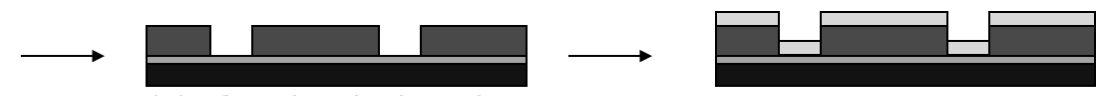

(f) a layer of metal is deposited over the whole substrate

(g) the remaining resist is stripped away, leaving the desired pattern

Figure A.1: An simple example of a photolithographic patterning process. 
1 Cleaning and treatment of substrate

2 Spin coating of photoresist

3 Pre-baking

4 Use of photomask and exposure of film

$5 \quad$ Post-processing and post-baking

6 Development

7 Hard-baking

8 Metal film deposition

$9 \quad$ Photoresist stripping (liftoff)

The concepts will be discussed more in detail in the following sections.

\section{Substrate Preparation}

Photoresists do not adhere well to the surface of silicon wafers; in general the more hydrophobic the surface, the better the adhesion. Several steps are, therefore, taken to increase this adhesion. The first is cleaning of the oxide surface. Contaminants such as solvent stains, oil and dust may be present on the surface and introduce defects into the process. For this reason the processing generally takes place in a clean room (a specially constructed, environmentally controlled, enclosed area that limits airborne contaminants). Depending on the needed tolerance for particle size, cleaning of the substrate can be as simple as using acetone and isopropyl alcohol to hydroflouric acid or piranha clean.

Water reduces adhesion, so a baking step is used to remove residual moisture. To prevent recurrence, reactive primers are used on the substrate. A commonly used primer is HMDS (hexamethyldisilazane) to promote adhesion between resists and oxides. The Si-NH-Si groups of HMDS react with the oxide by silylation forming a strong bond to the surface and blocking moisture. Additionally, the methyls of the HMDS bond strongly to the photoresist [171].

\section{Photomasks}

A photomask is essentially a light stencil which allows designed patterns to be formed in photoresist films by only allowing light to reach certain pre-defined regions. Photomasks can be made of near optically-flat fused silica or fused quartz, with a patterned absorber metal (generally chromium) 
$[171,173]$. Before exposure, the photomask is placed in contact or near-contact with the film. When exposure takes place, the areas patterned with the absorber metal completely block the radiation from reaching the photoresist film. This method is called shadow printing; other types of processes, such as projection printing, exist but are not discussed here [171].

\section{Photoresists}

Photoresists are polymers which are sensitive to, and undergo chemical processes under, UV radiation. The photoresist is spun, using a spin-coater, onto the substrate surface before exposure. Spinning makes use of centrifugal force to make the solution flow toward the edges of the substrate until it is flung off when the surface tension is exceeded [171]. The thickness of polymer deposited can be calculated using Equation A.1:

$$
T=\frac{K C^{\beta} \eta^{\gamma}}{\omega^{\alpha}}
$$

where $\mathrm{K}$ is a calibration constant, $\mathrm{C}$ is the polymer concentration in $(\mathrm{g} / 100 \mathrm{~mL}$ of solution), $\eta$ is the intrinsic viscosity and $\omega$ is the revolutions per minute. The exponents $\alpha, \beta$ and $\gamma$ need to be determined, but can then be used to predict $\mathrm{T}$ [171]. When purchasing a resist, this work has been done by the manufacturer who provide you with spin curves for their resists.

The state of the film after spin-coating is extremely important because defects in the film will propagate throughout the entire process. Too much or too little resist results in ridges or uncovered areas, respectively. In addition, dispensing of the resist can create bubbles which leave trails during spinning. After spin-coating, the resist may contain up to $15 \%$ solvent and internal stresses, which are undesirable. A pre-baking step is used to remove remaining solvent and stress and to promote adhesion of the photoresist to the substrate. Optimization of these steps involves parameters such as dispense rate, volume, spin speed, ambient temperature and humidity and will greatly increase yield [171].

There are many different photoresists, each optimized for a different film thickness, exposure wavelength or feature type and size; however, they fall basically into four types. First, a photoresist can be either one or two component. Single component resists have all the properties necessary for 
film development in a single polymer, namely photochemical reactivity, etch resistance and filmforming characteristics [172]. Two-component systems contain an inert resin serving as the binder and film-forming material as well as a sensitizer which is the photochemical material [172]. Second, resists have a tone that is either positive or negative and describes how the resist behaves under exposure. In positive photoresists, exposed areas become easier to etch during the development phase. In general this change occurs by main and side polymer chains being broken by photochemical processes during exposure $[171,172]$. In negative resists, exposed areas become more resistant to etching; normally this occurs by creation of cross-linking in the polymer [171, 172]. As examples of photoresists, poly(methyl methacrylate), or PMMA, is a single-component positive photoresist, while Kodak's KTFR is a two-component negative photoresist [171, 172].

\section{Exposure}

Exposure takes place after pre-baking and makes use of an aligning system that lines up the wafer with the features on the mask. This can be as simple as a UV lamp directly illuminating a mask in contact with the wafer to complicated micropositioning systems with illumination passing through lenses before reaching the mask/wafer. Care must be taken with the light to maintain proper characteristics. The light must have uniform intensity over the surface of the mask, have correct directionality and wavelengths (spectra) or defects and non-uniformities will be introduced into the photoresist film [171]. Lamps may be extreme UV (10-14 nm), deep UV (150-300 nm) or UV $(250-500 \mathrm{~nm})[171]$.

A useful metric for tracking or calculating the necessary exposure time is to considering the dose, $\mathrm{D}$, that the film receives. $\mathrm{D}\left(\mathrm{J} / \mathrm{cm}^{2}\right)$ is simply the intensity of the incident light $\left(\mathrm{W} / \mathrm{cm}^{2}\right)$ times the exposure time (s) [171].

The photochemical process induced by exposure may not complete during the exposure step. For certain resists, a post-exposure treatment is necessary before development. This can manifest as post-baking, reactive gas treatment, vacuum treatment, etc.. Optimization of both exposure and the post-treatment is crucial to proper development. 


\section{Development}

It is during development that the image is finally realized in the photoresist film. The film at this stage is a negative or positive image that serves as a stencil for the following additive or etching steps, however some experiments use the patterned resist itself as the final product.

Development takes advantage of the photochemically induced changes initiated during exposure to selectively etch away the more soluble regions of photoresist while leaving the less soluble regions intact. Development can be dry (vapor phase or plasma process) or wet (immersion or spray) [171]. Optimization is a must for this step. For example, if a positive photoresist is underdeveloped, the photoresist film will cover regions where it should not, serving to block the subsequent coating or etching steps. If overdeveloped, the features will become massively distorted as too much of the resist has been removed.

\section{Post-Development and Stripping}

To improve the film before use, there are two common procedures. One, called de-scumming, is removal of residual unwanted photoresist by exposing the film to a light oxygen plasma [171]. The other is called hard-baking; baking removes residual solvents in the film, further hardens and increases film adhesion to the substrate [171].

After the film is used during an additive or etching process, it must be removed for the final device. A photoresist resist stripper is used to do this, in a step commonly referred to as liftoff. Obviously the main concern is removing the film without damaging the device that is being constructed. Stripping can be done via dry (oxygen plasma, ashing) or wet (organic polymer etching) techniques [171].

\section{A.1.3 Our Application}

In the Yap Group we have mainly used three photoresists: Shipley SC1827 positive photoresist, Futurrex NR9G-1000PY negative resist and Futurrex PR1000A positive resist. The Michigan Tech MFF (Microfabrication Facility) is a Class 1000 cleanroom. There are four parameters out of our control that affect the development process: the MFF has (1) no temperature or (2) no humidity control, (3) the intensity of the UV lamp will vary with time and (4) the resists behave differently 
as they age. As these conditions change, the most affected parameters are the required exposure and development time, all other parameters should not require changing. With that in mind, the processing steps are almost identical for all three resist until development.

The general processes I used are described below.

1 Load the wafer into the spin-coater and spin the wafer. While spinning, spray rinse the wafer first with acetone and then isopropyl alcohols.

2 Remove the wafer and bake for at least two minutes (at $100{ }^{\circ} \mathrm{C}$ or greater) to remove residual moisture

3 Load the wafer into the spin-coater and select program run at $4000 \mathrm{rpm}$ for $60 \mathrm{~s}$.

4 Dispense HMDS onto the wafer surface (until covered) and spin for 30 - 40s.

5 Dispense the photoresist onto the wafer (until covered, no bubbles, but must be done relatively quickly) and spin for $60 \mathrm{~s}$ at $4000 \mathrm{rpm}$.

$6 \quad$ Remove the wafer and pre-bake at $120{ }^{\circ} \mathrm{C}$ for 2 minutes.

7 Set the EVG aligner (the UV exposer) to hard contact, with an exposure for 7 - 12 seconds (depending on the current conditions).

8 For the negative resist, post-bake at $120{ }^{\circ} \mathrm{C}$ for two minutes; no post-bake is necessary for the positive resists.

9-NR A 1:1 volume ratio of deionized water and Futurrex developer RD6 are used for development, with very short development times. The lowest on record is 4 seconds, usually between 8-12 seconds but occasionally longer.

9-PR MF319 developer is used, generally 20 - 120s.

10 Metal coating, generally by sputtering.

11 Lift-off is easily done using acetone. It may require mechanical scrubbing or sonication to complete lift-off.

As of the time of this writing, we are moving on to a dual-resist method that results in easier, cleaner lift-off. 


\section{Etching}

For etching processes, such as the ones used to make the molecular junctions, the steps are similar. The biggest difference is that after the substrates are cleaned, the metal films are deposited first. Then, the photolithography is done as usual, only the reverse resist tone is used such that the regions that are to be the electrodes are covered after development. At this point, it is simply a matter of submersion in the relevant etchant for the metal film. The etchants have a rate associated with them (such as $3 \mathrm{~nm} / \mathrm{s}$ ) which tells you the minimum needed time to leave the substrate in the solution. After etching is complete, the substrate must be rinsed with water. Then, a bath in PR stripper will remove the resist and complete the process.

\section{A.2 Raman Spectra [174]}

When interacting with matter, light can either be absorbed or scattered. In order for absorbtion to occur, the energy of the incoming photon must be the same as the amount of energy between the ground state of the molecule and one of its excited states. Scattering, however, can occur whether or not the photon's energy matches one of the excited states. Further, the interaction between the light and the molecule is different for scattering than it is for absorbtion.

Two types of scattering are easily identified. The most intense form is Rayleigh scattering and occurs when the electron cloud interacts and relaxes without nuclear movement. Rayleigh scattering is an essentially elastic interaction with no appreciable change in photon energy. The other is Raman scattering, with only one in $10^{6}$ to $10^{8}$ photons scattered in this way. In Raman scattering, during the photon-electron interaction, the nuclei begin to move. Because nuclei are relatively heavy, this results in a significant shift in energy of the photon as it leaves the molecule. This shift in energy of the photon can be positive or negative depending on whether the molecule started in the ground state (Stokes) or an excited state (anti-Stokes). Since most molecules are in the ground state at room temperature, most observed Raman scattering is Stokes Raman scattering

A light wave can be described as an oscillating dipole that propagates through space. When passing over a molecule, the dipole distorts the electron clouds around the nuclei. When the energy is released again, the photon is said to have been scattered. The process differs from absorbtion in three ways. First, the additional energy does not promote an electron to any specific excited state 


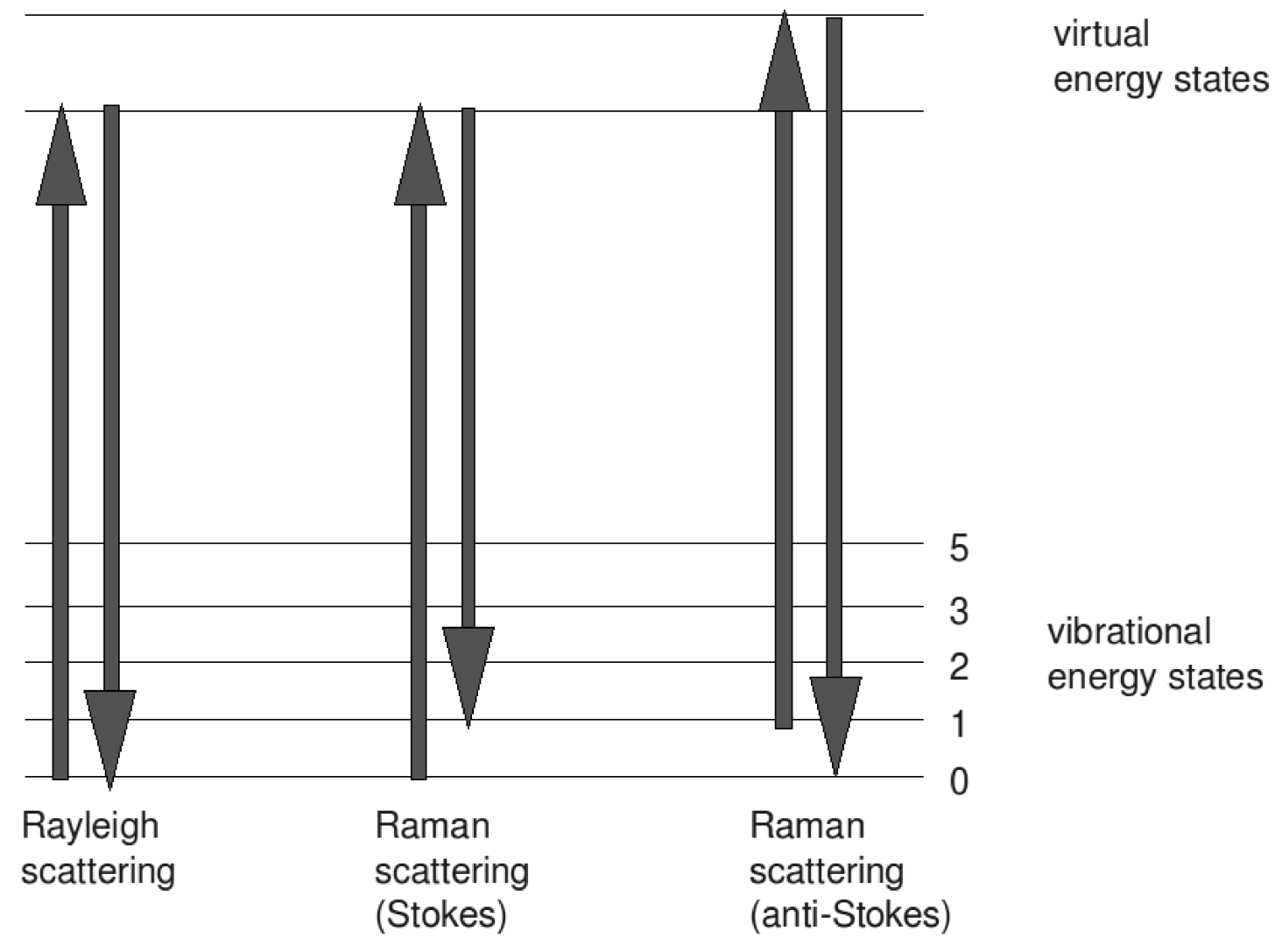

Figure A.2: Energy level diagrams for Rayleigh and Raman scattering processes.

of the molecule; all states are involved to different extents to form this state. The energy of this state is dependent on the energy of the laser used and the amount of distortion is dependent on the electronic properties of the molecule and on the energy of the laser. Second, the lifetime of this excited state is very short compared to most absorbtion processes. Third, there is a link between the polarization of the exciting and scattered photons, which can be of value in assigning vibrations to peaks. A diagram comparing the excitations of Rayleigh and Raman scattering is shown in Figure A.2.

Raman scattering should be expressed as a shift in wavelength (and therefore energy) as $\Delta \mathrm{cm}^{-1}$, but the conventional notation is simply $\mathrm{cm}^{-1}$. The general range of interest is in the range of 400 $\mathrm{cm}^{-1}$ to $3600 \mathrm{~cm}^{-1}$, which includes most characteristic molecular modes. Most Raman spectrometers are incapable of taking data below $200 \mathrm{~cm}^{-1}$ because of the filters used to block the laser light. 
Thomas Ebbeson, Hidefumi Hiura and their colleagues at NEC conducted the first Raman experiments on MWCNTs [175]. The experiments compared material containing CNTs and carbon nanoparticles from the core of the cathode with those from those from the outer shell of the cathodic deposit and highly organized pyrolytic graphite (HOPG) [13]. The MWCNT spectra shares a peak in the neighborhood of $1580 \mathrm{~cm}^{-1}$ corresponding to the $\mathrm{E}_{2 g}$ vibrations of graphite; this is often referred to as the "graphitic" peak. The bands around $1350 \mathrm{~cm}^{-1}$ are attributed to disorder in the crystal lattice [13]. Comparison of the intensities of these two peaks is a quick check of the crystallinity of CNTs without the need for SEM. When making this comparison, the ratio of graphitic peak intensity to disorder intensity is often cited, and is abbreviated G/D ratio. Because of the nature of the differing chemical bonds, Raman can be used also to check to see if functionalities have been added to the tubes, or whether or not doping has taken place.

\section{A.3 Scanning Electron Microscope [176]}

\section{A.3.1 Historical Perspective}

The first recognized description of a scanning electron microscope (SEM) was done by Knoll and Ruska as far back as 1935. The first SEM used to examine thick specimens was performed by Zworykin et al. in 1942. They realized secondary electron emission would result in topological contrast and performed detailed important analysis of the interrelation of lens aberrations, gun brightness and spot size. Using electron multiplier tubes as preamplifiers to enhance the secondary electron signal, they were able to reach resolutions of $50 \mathrm{~nm}$ with the first true modern SEM. However, this resolution was behind the transmission electron microscope (TEM) in development at the time, and interest waned. Throughout the 1950's incremental improvements were made to many of the subsystems of the SEM. In 1963, Pease built an SEM that was the prototype of the commercial systems which followed, called the SEM V. Since the first commercial SEM, the Cambridge Scientific Instruments Mark I "Stereoscan," more than 50,000 SEMs have been sold. More modern improvements include field emitting sources, more efficient detectors, image processing and digital storage. 


\section{A.3.2 Background}

The scanning electron microscope is an indispensable instrument for nanotechnology. Inside the SEM, a sample area that is to be examined is irradiated with a highly focused electron beam. In order to form images, the beam is rastered over a given area, though for other analysis techniques the beam may be stationary. The interaction of the beam with the sample causes the emission of many kinds of signals, including characteristic x-rays, scattered electrons and secondary electrons. It is the electrons that are the most important for imaging and which provide the intensity signal for each pixel in the image.

Because of the very small spread of the electron beam, scanning electron microscopes are capable of extremely high resolutions, with high end machines capable of resolutions of a few nanometers. The depth of field is large on SEMs, especially compared to optical microscopes, and therefore samples with large height variations are able to be imaged in focus all at once. The large depth of field is shown in Figure A.3. Though powerful, SEMs have requirements that substrates can be put under high vacuum without damage to the machine or themselves, and if a sample is not conductive it can be difficult to image without charge build-up causing distortion in the image. In my research, the SEM is used for topological imaging. While a thorough description of the inner workings and tuning of the SEM is too long to include in this appendix, the basic concepts are discussed below. For those interested in more information, I suggest the text used for this section, reference [176].

The microscope column itself is composed of the following parts, as shown in Figure A.4: Electron Gun

Electron Lens (1st Condenser)

Spray Aperture

Scan Coils

Magnification Control and Scan Generator

Final Lens Aperture

Detector

The electron gun is the source of the electrons and accelerates them to a voltage of $0.1-30$ 


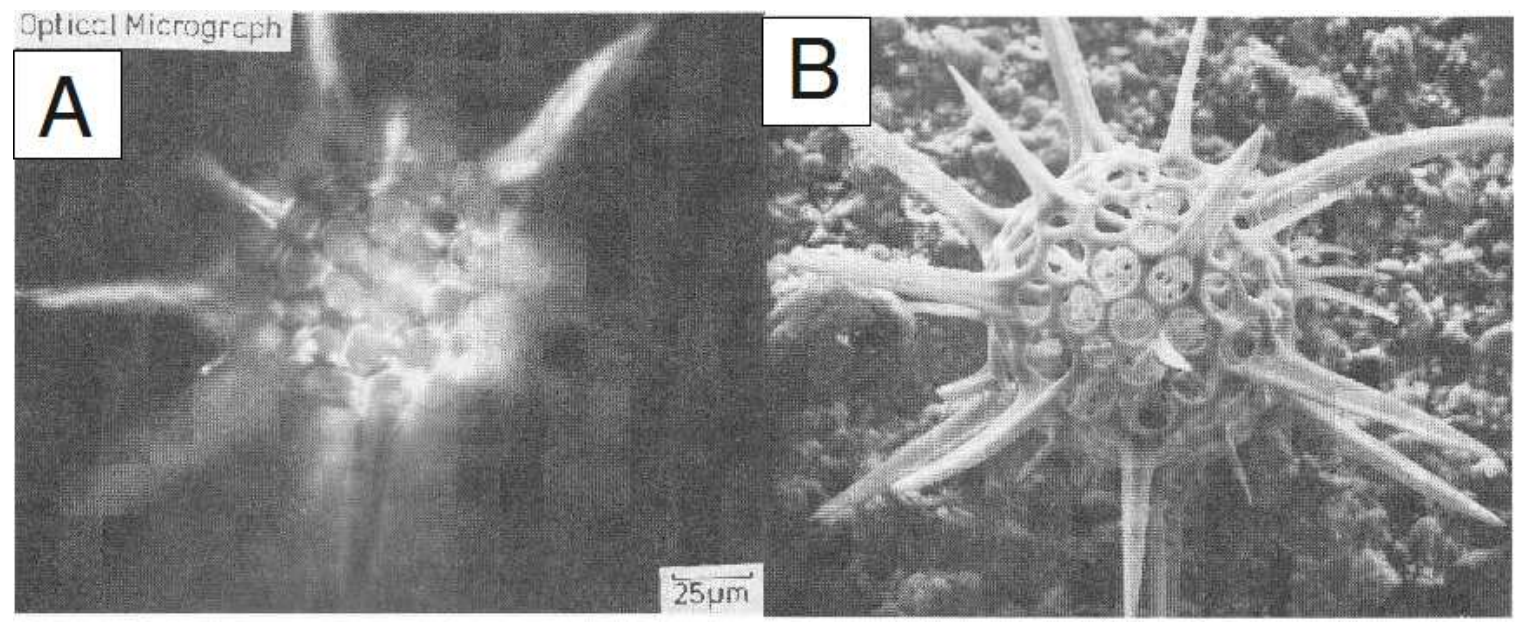

Figure A.3: (a)Optical microscope image showing distortion due to very small depth of field. (b)SEM image of the same object completely in focus due to the large depth of field. Reprinted from Reference [176](Figure 1.3) with the kind permission of Springer Science and Business Media.

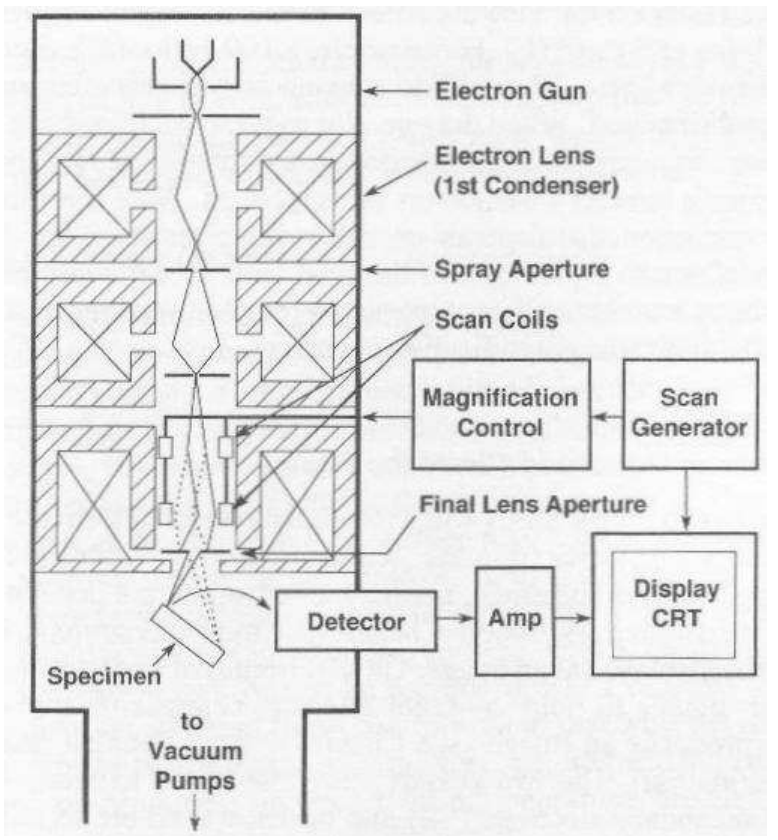

Figure A.4: Schematic of the column of an SEM. Reprinted from Reference [176](Figure 2.2) with kind permission of Springer Science and Business Media. 


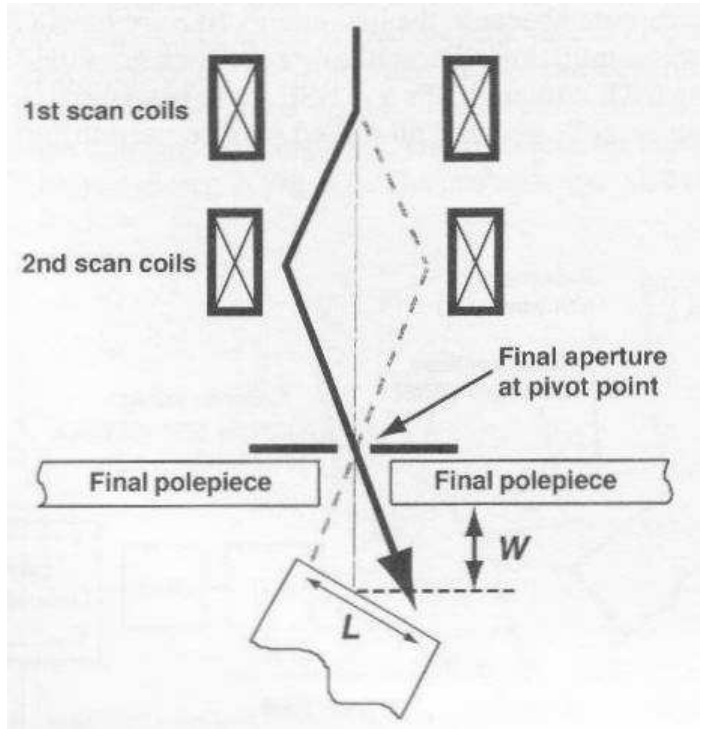

Figure A.5: Schematic showing how the coils raster the beam in order to scan the surface of a sample. Reprinted from Reference [176](Figure 2.3) with kind permission of Springer Science and Business Media.

$\mathrm{keV}$. The beam at this stage is not at all focused, so electron lenses are used to demagnify the beam. Apertures further reduce the diameter of the beam, leading to lower current signal but higher resolution. A series of scan coils is used to deflect the beam so it can be moved along the sample surface. Two pairs of electromagnetic scan coils do the job; the first pair deflects the beam off the optical axis while the second pair bends it back at the pivot point of the scan, shown in more detail in Figure A.5. This system is also responsible for the magnification, since the magnification is simply the ratio of the length of the raster on the viewing screen to the length of the raster on the specimen. Once the beam strikes the target, electrons are scattered and ejected and collected by the detector. Not all points on the substrate generate the same signal, and this provides contrast in the image. This contrast can come from a variety of different reasons, among them changes in topology, density and conductivity. Other signals can be collected from the sample to do different types of analysis, but they are not relevant to the work done here and will not be discussed.

The SEM used for this research is a Hitachi S-4700 field emission scanning electron microscope (FESEM). During optimal tuning and conditions, the S-4700 is capable of reaching magnifications of 100,000x. Accelerating voltages used are generally $2-20 \mathrm{kV}$. 


\section{Appendix B}

\section{Publications \& Awards}

\section{Publications}

1. Jason Moscatello, Vijaya Kayastha, Benjamin Ulmen, Archana Pandey, Shun Wu, Abhay Singh, and Yoke Khin Yap. "Surfactant-free dielectrophoretic deposition of multi-walled carbon nanotubes with tunable deposition density," Carbon (Accepted May 2010)

2. (Under review) Archana Pandey, Abishek Prasad, Jason Moscatello and Yoke Khin Yap. "PMMA-CNT Matrices for High-Performing Electron Field Emitters," Advanced Functional Materials (2010)

3. Archana Pandey, Abhishek Prasad, Jason Moscatello, Yoke Khin Yap, "Comparing Field Emission Stability of Lithography-free, Modified Multi-walled Carbon Nanotubes," Mater. Res. Soc. Symp. Proc., Volume 1204, 1204-K18-22 (2010)

4. Archana Pandey, Abhishek Prasad, Jason Moscatello, Benjamin Ulmen and Yoke Khin Yap, "Enhanced field emission stability and density produced by conical bundles of catalyst-free carbon nanotubes," Carbon, 48, 287 (2010)

5. Jason Moscatello et al., "Controlled Growth of Carbon, Boron Nitride, and Zinc Oxide Nanotubes," Invited Paper in Special Issue on Nanosensors for Defense $\&$ Security, IEEE Sensors Journal 8, $922(2008)$ 
6. Jason Moscatello, Vijaya Kayastha, Archana Pandey, Benjamin Ulmen and Yoke Khin Yap, "Dielectrophoretic Deposition of Carbon Nanotubes with Controllable Density and Alignment," Mater. Res. Soc. Symp. Proc., Vol. 1057, Paper 1057-II15-07 (2008)

7. Vijaya Kayastha, Shun Wu, Jason Moscatello, Yoke Khin Yap, "Synthesis of Vertically Aligned Single- and Double-Walled Carbon Nanotubes without Etching Agents," J. Phys. Chem. C. (Letter), 111, 10158 (2007)

8. Jason Moscatello et al., "Growth of Carbon, Boron Nitride and ZnO Nanotubes for Biosensors," Invited paper in ECS Transactions 3, 1 (2007)

\section{Awards}

1. Michigan Tech Finishing Fellowship - Awarded tuition and $\$ 6000$ stipend for Spring 2009

2. DeVlieg Foundation Fellowship - One time award of $\$ 3000,2007$ 
Appendix C

\section{Permissions}




\title{
C.1 American Institute of Physics, Reference [43]
}

\begin{abstract}
Re: Request Permission for
Reprint

From: Rights@aip.org

To: jpmoscat@mtu.edu

Dear Dr. Moscatello:

Thank you for requesting permission to reproduce material from American

Institute of Physics publications.

Permission is granted - subject to the conditions outlined below - for the following:

Figure 3

V.K. Kayastha, Y.K. Yap, S. Dimovski and Y. Gogotsi; Controlling dissociative adsorption for effective growth of carbon nanotubes; Appl. Phys. Lett. 85:3265 (2004)

To be used in the following manner:

Included as part of your Ph.D. dissertation for submission to Michigan Tech University.

1. The American Institute of Physics grants you the right to reproduce the material indicated above on a one-time, non-exclusive basis, solely for the purpose described. Permission must be requested separately for any future or additional use.

2. This permission pertains only to print use and its electronic equivalent, including CD-ROM or DVD.

3. The following copyright notice must appear with the material (please fill in the information indicated by capital letters): "Reprinted with permission from [FULL CITATION]. Copyright [PUBLICATION YEAR], American Institute of Physics."

Full citation format is as follows: Author names, journal title, Vol. \#, Page \#, (Year of publication).

For an article, the copyright notice must be printed on the first page of the article or book chapter. For figures, photographs, covers, or tables, the notice may appear with the material, in a footnote, or in the reference list.

4. This permission does not apply to any materials credited to sources other than the copyright holder.

5. If you have not already done so, please attempt to obtain permission from at least one of the authors. The author's address can be obtained from the article.
\end{abstract}

Tue 19 Jan 2010 12:21:28 PM

EST 
Please let us know if you have any questions.

Sincerely,

Susann Brailey

Office of the Publisher, Journals and Technical Publications

Rights \& Permissions

American Institute of Physics

Suite 1N01

2 Huntington Quadrangle

Melville, NY 11747-4502

516-576-2268 TEL

516-576-2450 FAX

rights@aip.org

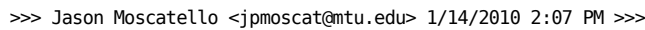
office of Rights and Permissions:

I would like to request permission to reprint a figure from one of your journal articles for use in my Ph.D. dissertation. The paper is from my research group, and the citation is:

V.K. Kayastha, Y.K. Yap, S. Dimovski and Y. Gogotsi; Controlling dissociative adsorption for effective growth of carbon nanotubes; Appl. Phys. Lett. 85:3265 (2004)

I would like to use figure 3 :

"FIG. 3. (a) Sequences of dissociative adsorption of $\mathrm{C} 2 \mathrm{H} 2$ on Fe surface. The (b) decomposed carbon atoms (c) diffused into the Fe nanoparticle until (d) supersaturation and (e) segregate as nanotubes."

The dissertation is to be printed internally at Michigan Tech and is not for profit. Thank you for your help and for your time.

Sincerely,

Jason Moscatello 


\section{C.2 American Institute of Physics, Reference [155]}

AMERICAN INSTITUTE OF PHYSICS LICENSE TERMS AND CONDITIONS

Mar 24, 2010

This is a License Agreement between Jason Moscatello ("You") and American Institute of Physics ("American Institute of Physics") provided by Copyright Clearance Center ("CCC"). The license consists of your order details, the terms and conditions provided by American Institute of Physics, and the payment terms and conditions.

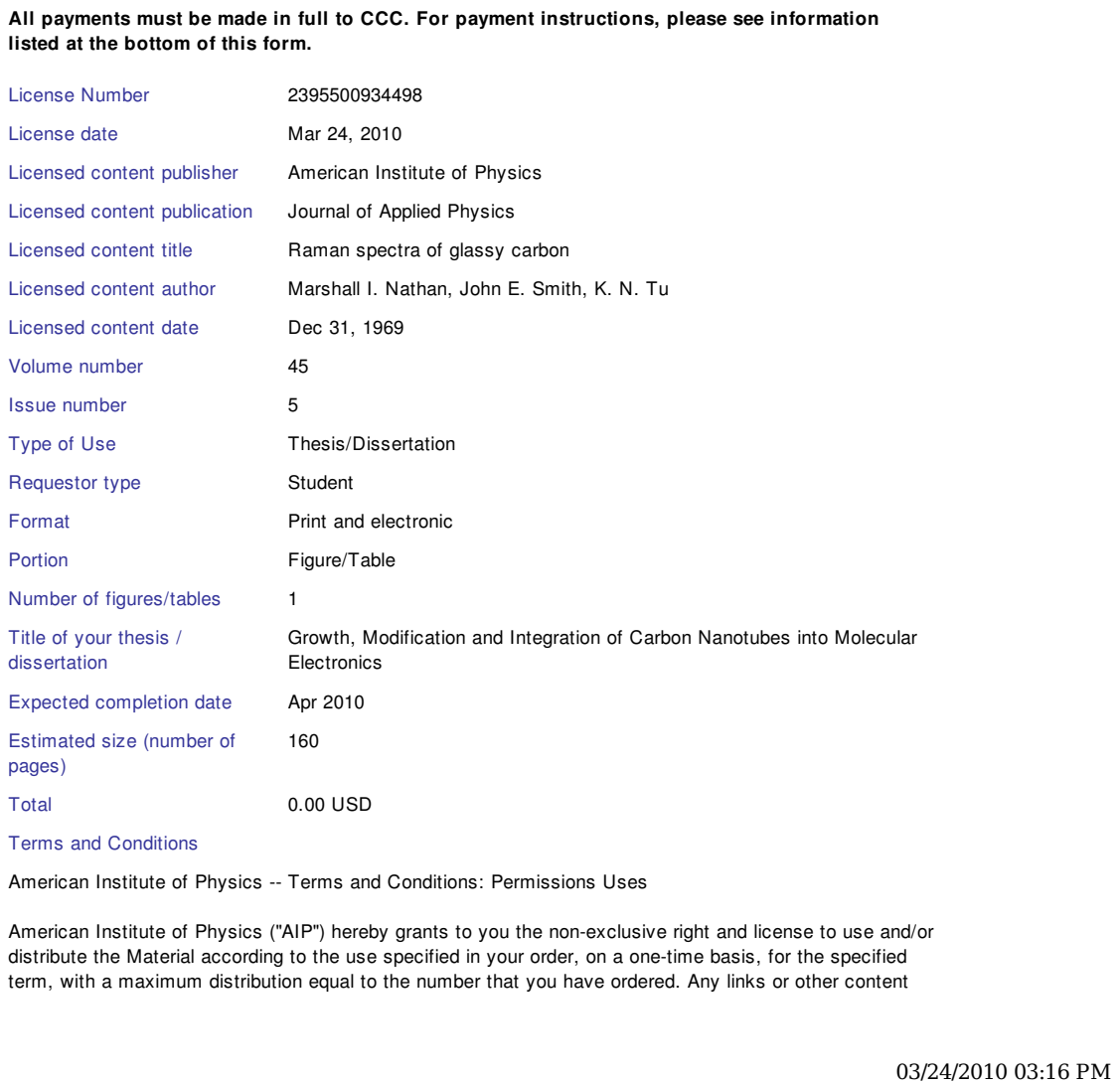


accompanying the Material are not the subject of this license.

1. You agree to include the following copyright and permission notice with the reproduction of the Material: "Reprinted with permission from [FULL CITATION]. Copyright [PUBLICATION YEAR],

American Institute of Physics." For an article, the copyright and permission notice must be printed on the first page of the article or book chapter. For photographs, covers, or tables, the copyright and permission notice may appear with the Material, in a footnote, or in the reference list.

2. If you have licensed reuse of a figure, photograph, cover, or table, it is your responsibility to ensure that the material is original to AIP and does not contain the copyright of another entity, and that the copyright notice of the figure, photograph, cover, or table does not indicate that it was reprinted by AIP, with permission, from another source. Under no circumstances does AIP, purport or intend to grant permission to reuse material to which it does not hold copyright.

3. You may not alter or modify the Material in any manner. You may translate the Material into another language only if you have licensed translation rights. You may not use the Material for promotional purposes. AIP reserves all rights not specifically granted herein.

4. The foregoing license shall not take effect unless and until AIP or its agent, Copyright Clearance Center, receives the Payment in accordance with Copyright Clearance Center Billing and Payment Terms and Conditions, which are incorporated herein by reference.

5. AIP or the Copyright Clearance Center may, within two business days of granting this license, revoke the license for any reason whatsoever, with a full refund payable to you. Should you violate the terms of this license at any time, AIP, American Institute of Physics, or Copyright Clearance Center may revoke the license with no refund to you. Notice of such revocation will be made using the contact information provided by you. Failure to receive such notice will not nullify the revocation.

6. AIP makes no representations or warranties with respect to the Material. You agree to indemnify and hold harmless AIP, American Institute of Physics, and their officers, directors, employees or agents from and against any and all claims arising out of your use of the Material other than as specifically authorized herein.

7. The permission granted herein is personal to you and is not transferable or assignable without the prior written permission of AIP. This license may not be amended except in a writing signed 
by the party to be charged.

8. If purchase orders, acknowledgments or check endorsements are issued on any forms containing terms and conditions which are inconsistent with these provisions, such inconsistent terms and conditions shall be of no force and effect. This document, including the CCC Billing and Payment Terms and Conditions, shall be the entire agreement between the parties relating to the subject matter hereof.

This Agreement shall be governed by and construed in accordance with the laws of the State of New York. Both parties hereby submit to the jurisdiction of the courts of New York County for purposes of resolving any disputes that may arise hereunder.

Gratis licenses (referencing $\$ 0$ in the Total field) are free. Please retain this printable license for your reference. No payment is required.

If you would like to pay for this license now, please remit this license along with your payment made payable to "COPYRIGHT CLEARANCE CENTER" otherwise you will be invoiced within 48 hours of the license date. Payment should be in the form of a check or money order referencing your account number and this invoice number RLNK10756934.

Once you receive your invoice for this order, you may pay your invoice by credit card. Please

follow instructions provided at that time.

Make Payment To:

Copyright Clearance Center

Dept 001

P.O. Box 843006

Boston, MA 02284-3006

If you find copyrighted material related to this license will not be used and wish to cancel, please contact us referencing this license number 2395500934498 and noting the reason for cancellation.

Questions? customercare@copyright.com or +1-877-622-5543 (toll free in the US) or +1-978-646-2777. 


\section{C.3 Archana Pandey}

jpmoscat@mtu.edu

Re: Permission

Fri 11 Jun 2010 11:30:01 AM EDT

From: arpandey@mtu.edu

To:jpmoscat@mtu.edu

Hi Jason,

Sure. You can use my data for your thesis. I grant Jason Moscatello permission to use my data from my research work. You may reproduce or modify the figure in any way for use in your thesis work.

Thanks,

Archana

-...- Original Message .....

From: "Jason Moscatello" <jpmoscat@mtu.edu>

To: "Archana Pandey" <arpandey@mtu.edu>

Sent: Friday, June 11, 2010 11:17:43 AM GMT -05:00 US/Canada Eastern

Subject: Permission

Archana,

I need permission from you to use your material, the grad school said I am missing it. Can you type me up a permission please?

-jason-

-Archana

Live as if you were to die tomorrow... Learn as if you were to live forever... 


\title{
C.4 Benjamin Ulmen, Reference [81]
}

\author{
Zimbra: jpmoscat@mtu.edu \\ https://huskymail.mtu.edu/ \\ Zimbra Collaboration Suite \\ jpmoscat@mtu.edu \\ Thesis figures \\ Mon 18 Jan 2010 07:24:54 PM EST \\ From: bulmen2@illinois.edu \\ To: jpmoscat@mtu.edu \\ I grant Jason Moscatello permission to use the figure of the PE-CVD from my M.S thesis at \\ Michigan Technological University. He may reproduce or modify the figure in any way for use \\ in his thesis work. \\ Signed, \\ Ben Ulmen
}


From: bulmen2@illinois.edu

To: jpmoscat@mtu.edu

I, Ben Ulmen, being of sound mind and body, do hereby grant Jason Moscatello the right to use the figure of the field emission chamber and PECVD from my thesis. ;

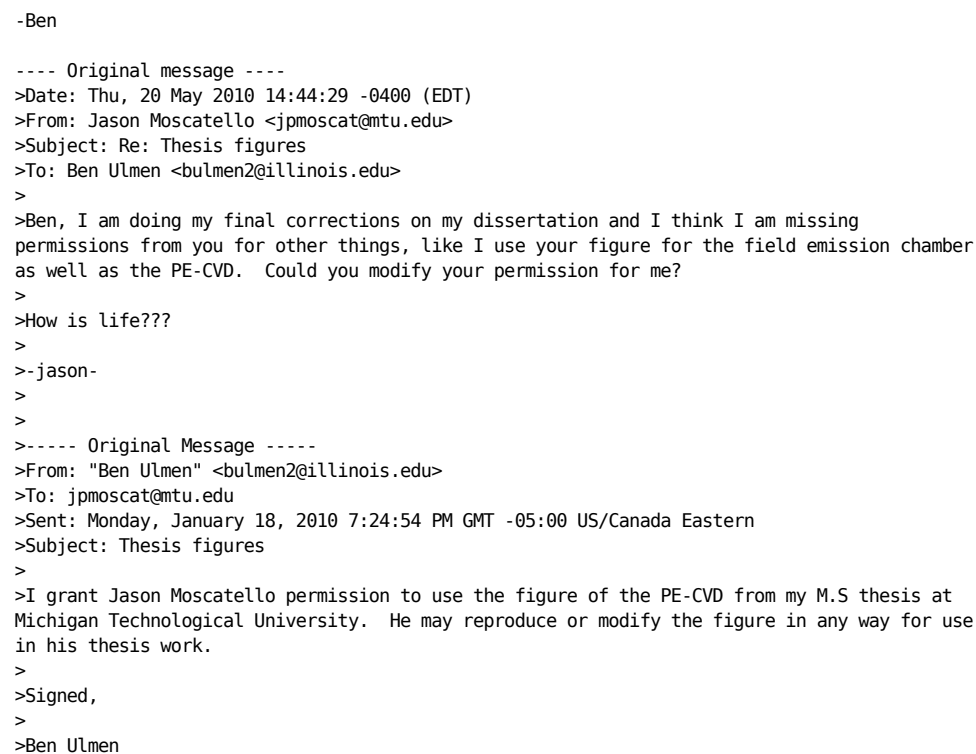




\title{
C.5 Electrochemical Society, Reference [153]
}

\author{
Request for Permission to Reproduce or Re-Publish ECS Material
}

Please fax this form to: The Electrochemical Society (ECS). Attn: Permissions Requests, 1.609.737.2743.

You may also e-mail your request to: conyrightaelectrochem.org. Inelude all the information as required on this

form. Please allow 3-7 days for your request to be processed.

1 am preparing a (choose one): $\square$ paper $\square$ chapter $\square$ book thesis

entitled: Growth, Modification and Integration of Carbon Nanotubes into Molecular Electronics

to be published by: Michigan Technological University

in an upcoming publication entitled:

1 request permission to use the following material in the publication noted above, and request nonexclusise rights for all subsequent editions and in all foreign language translations for distribution throughout the world.

Description of material to be used - Indicate what material you wish to use (figures, tables, text, etc.) and give the full bibliographic reference for the source publication. You may attach a separate list, organized by ECS title.

Ali Osman Solak, Srikanth Ranganathan, Takashi Itoh and Richard L. McCreery. A mechanism for conductance switching in carbon-based molecular electronic junctions. Electrochemical and Solid-State Letters. 5(8):E43-E46 2002. Figure 2.

Signature: Date: March 24, 2010

Name: Jason P. Moscatello

Address: Michigan Technological University

118 Fisher Hall, 1400 Townsend Drive

Houghton, MI 49931

Telephone: $906-487-2086$ Fax: $906-487-2933$

E-mail: jpmoscat@mtu.edu

Permission is granted to reproduce the above-referenced material. Please acknowledge the author(s) and publication data of the original material, and include the words: "Reproduced by permission of The

Electrochemical Society."

April 10,2010

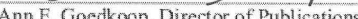




\section{C.6 Elsevier, Reference [32]}

Zimbra: jpmoscat@mtu.edu

https://huskymail.mtu.edu/

\begin{tabular}{l} 
Zimbra Collaboration Suite \\
\hline Permission granted \\
From: K.Harris@elsevier.com \\
To: jpmoscat@mtu.edu \\
Attachments: ole0.bmp (18.9KB)
\end{tabular}

jpmoscat@mtu.edu

Wed 03 Mar 2010 06:09:59 AM EST 


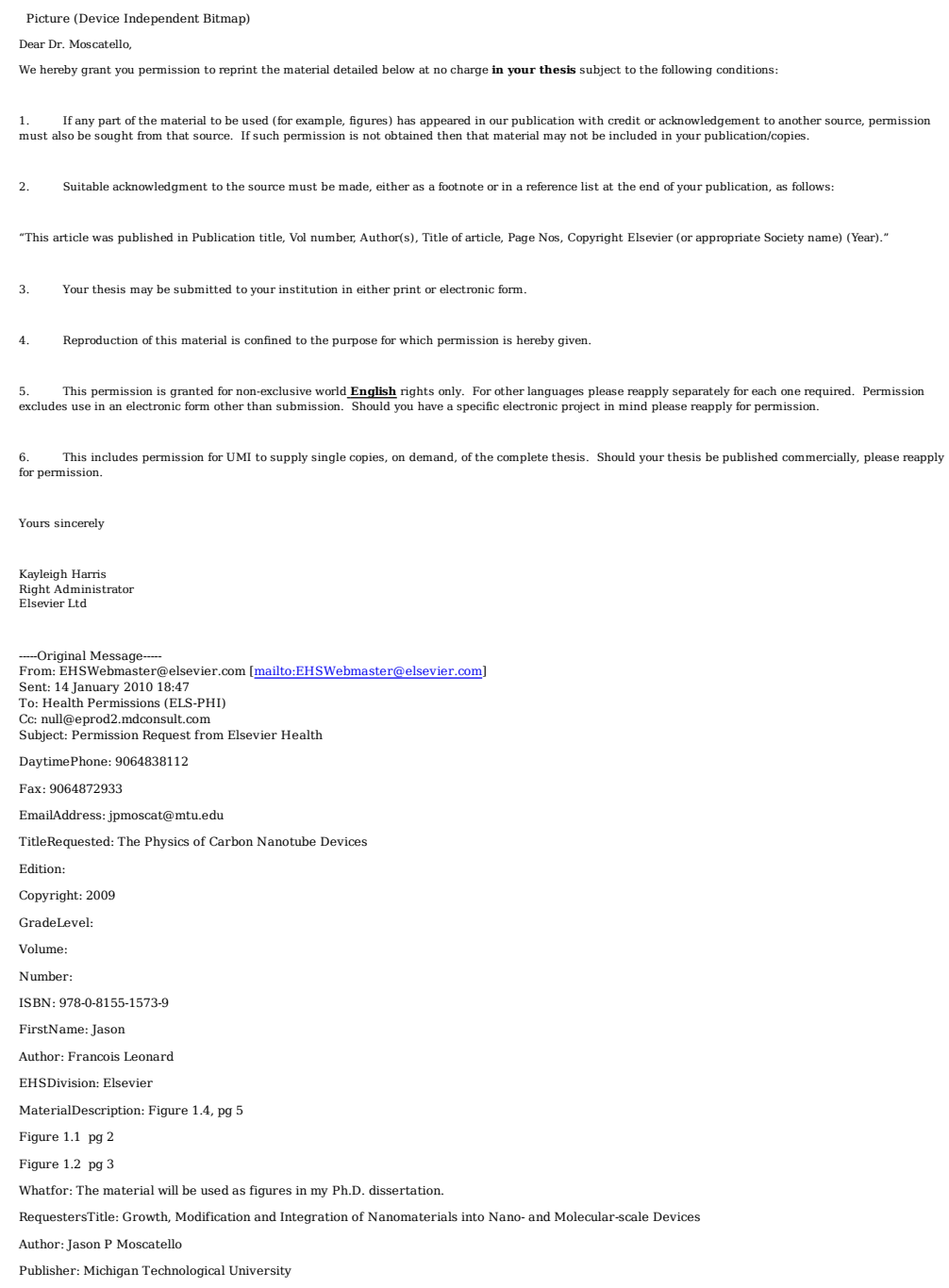




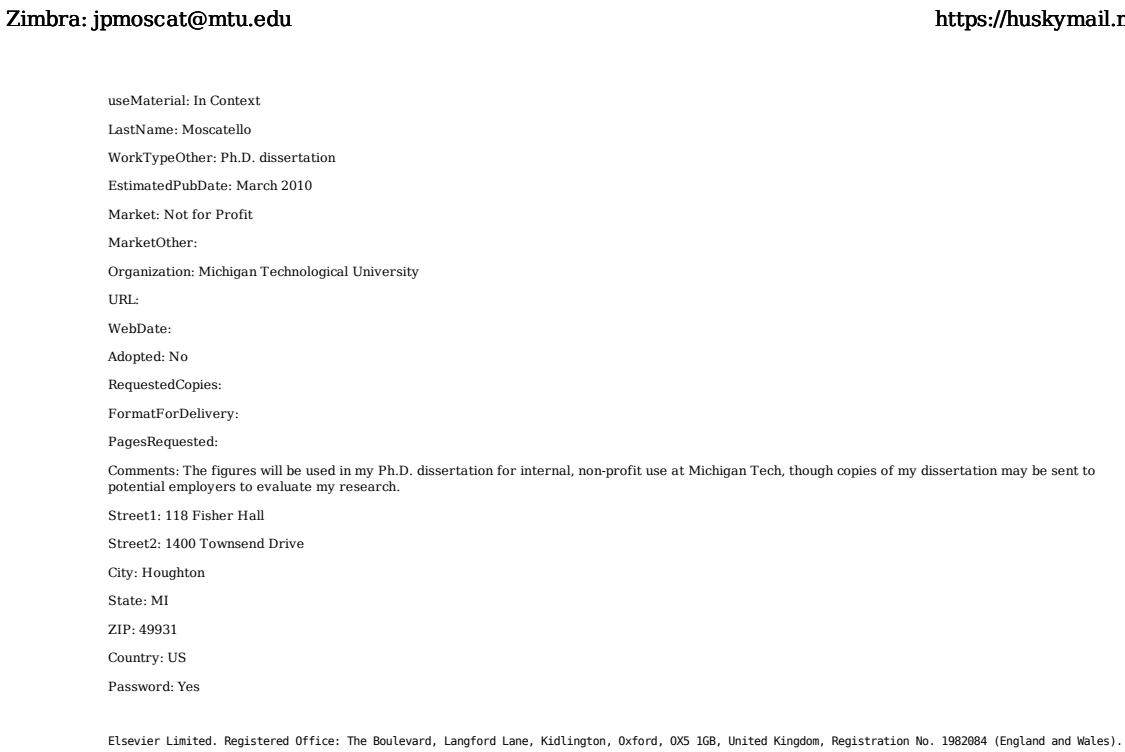




\section{C.6.1 Author's Permission}

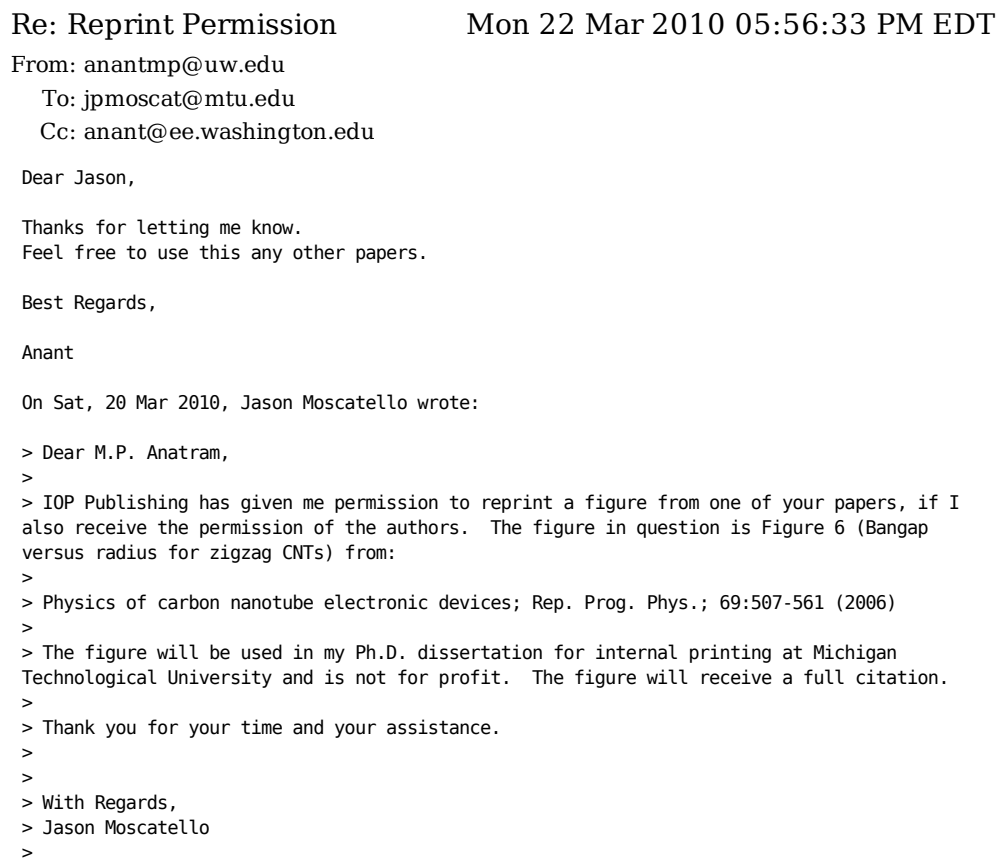




\section{C.7 IEEE, Reference [67]}

Permission you requested from $\quad$ Wed 26 May 2010 10:12:00 AM
IPR

From: IPR@ieee.org

To: jpmoscat@mtu.edu

Dear Mr. Jason Moscatello,

You have requested permission to use figures from the IEEE-copyrighted paper entitled:

"Controlled Growth of carbon, boron nitride and zinc oxide nanotubes"

By J. Moscatello et al

That was published in: Sensors Journal, IEEE

Aug. 2008

The above IEEE content will be reused in a thesis entitled:

thesis by Michigan Tec $\mathrm{h}$ on $01-07-2010$

We are happy to grant you permission to reuse the IEEE copyrighted figures requested in all electronic and printed formats.

Our only requirements are that you credit the original source (author, paper, and publication), and that the IEEE copyright line (Copyright [Year] IEEE) appears prominently with each figure.

You may also choose to print a copy for your records.

If you have any questions concerning this permission grant, please use the letter's reference number [ 0000007547] in all correspondence and contact Jacqueline Hansson at $+1-732-562-3828$ (phone) or send an email to $j$.hansson@ieee.org

Sincerely,

Jacqueline Hansson

IEEE Intellectual Property Rights Coordinator

IEEE Intellectual Property Rights Office

445 Hoes Lane, Piscataway NJ 08854

$+1-732-562-3828$ 


\section{C.8 Imperial College Press, Reference [31]}

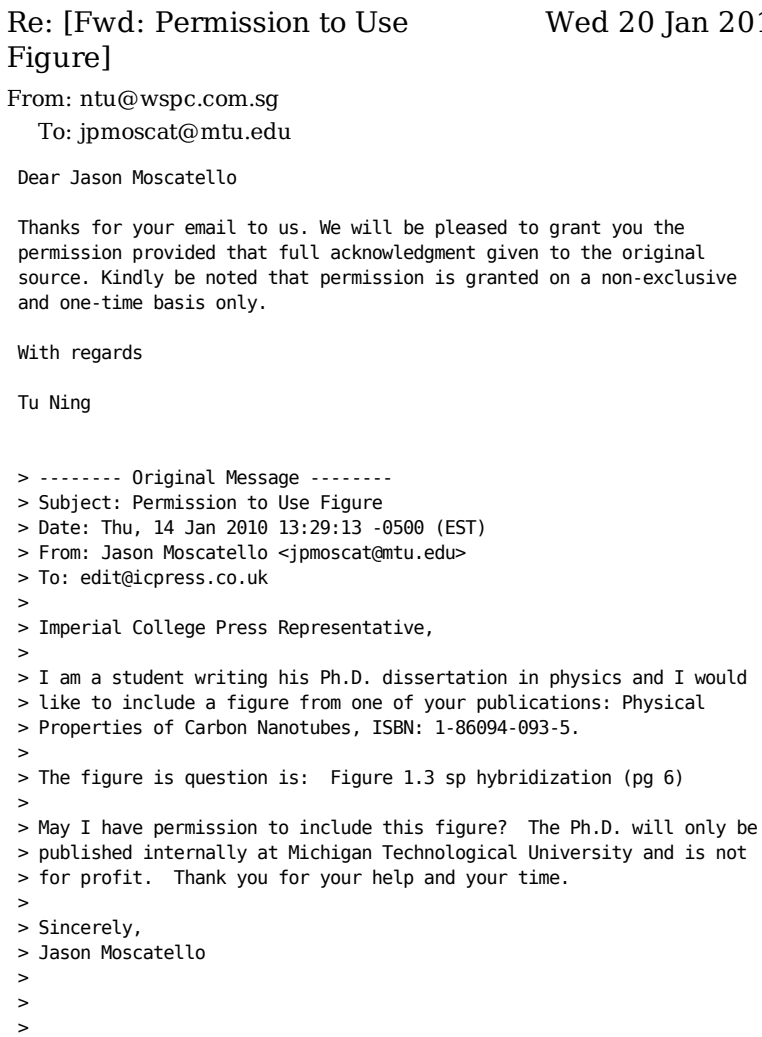




\section{C.9 Institute of Physics, Reference [35]}

\begin{tabular}{|c|c|c|c|}
\hline 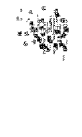 & 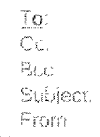 & \multicolumn{2}{|c|}{ PermissionsilOPP@IOPP, } \\
\hline 4 & \multicolumn{3}{|c|}{$\begin{array}{l}\text { Jason Moscatello } \\
\text { <jpmoscat@mtu.edu> } \\
\text { 01/14/2010 01:58 PM }\end{array}$} \\
\hline & & & ject Request for permission to reprint figure \\
\hline
\end{tabular}

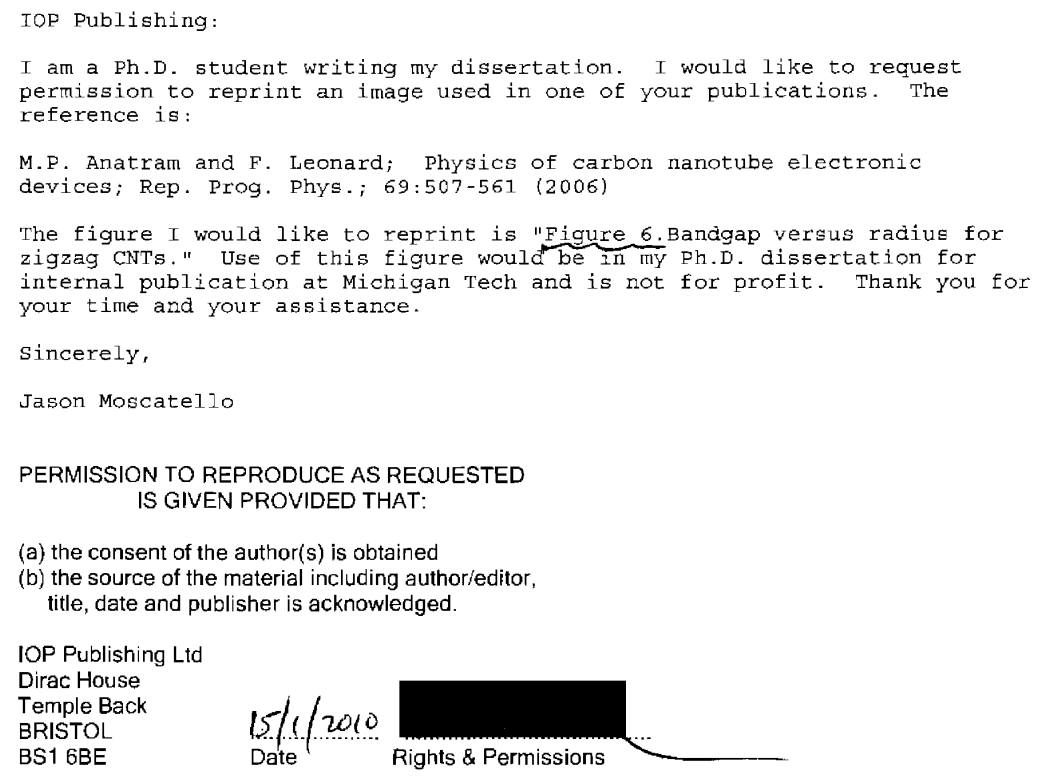




\section{C.9.1 Author's Permission 1}

Re: Reprint Permission

Sat 20 Mar 2010 07:56:49 PM EDT

From: fleonar@sandia.gov

To: jpmoscat@mtu.edu

No problem.

-Francois

..... Original Message ......

From: Jason Moscatello <jpmoscat@mtu.edu>

To: anant@arc. nasa.gov <anant@arc. nasa.gov>

Cc: Leonard, Francois

Sent: Sat Mar 20 15:09:50 2010

Subject: Reprint Permission

Dear M.P. Anatram and F. Léonard,

IOP Publishing has given me permission to reprint a figure from one of your papers, if I also receive the permission of the authors. The figure in question is Figure 6 (Bangap versus radius for zigzag (NTs) from:

Physics of carbon nanotube electronic devices; Rep. Prog. Phys.; 69:507-561 (2006)

The figure will be used in my Ph.D. dissertation for internal printing at Michigan

Technological University and is not for profit. The figure will receive a full citation.

Thank you for your time and your assistance.

With Regards,

Jason Moscatello 


\section{C.9.2 Author's Permission 2}

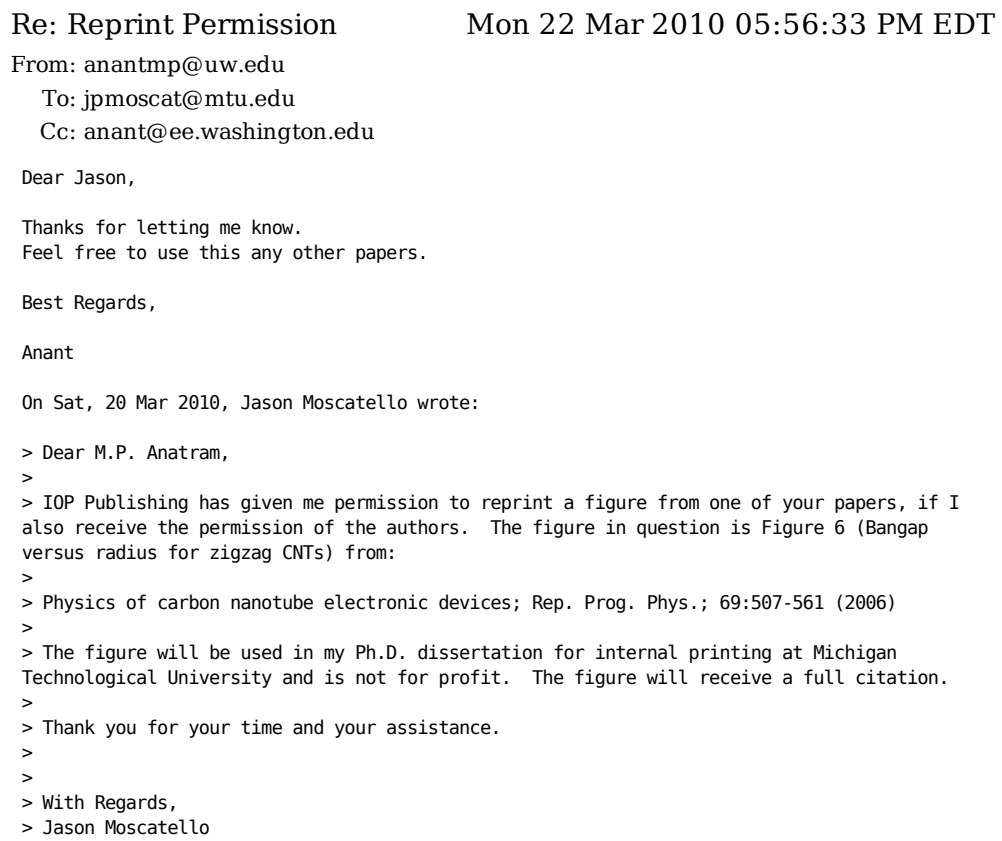




\title{
C.10 Ravi Chintala, Reference [167]
}

\author{
Zimbra: jpmoscat@mtu.edu \\ https://huskymail.mtu.edu/ \\ Zimbra Collaboration Suite \\ jpmoscat@mtu.edu

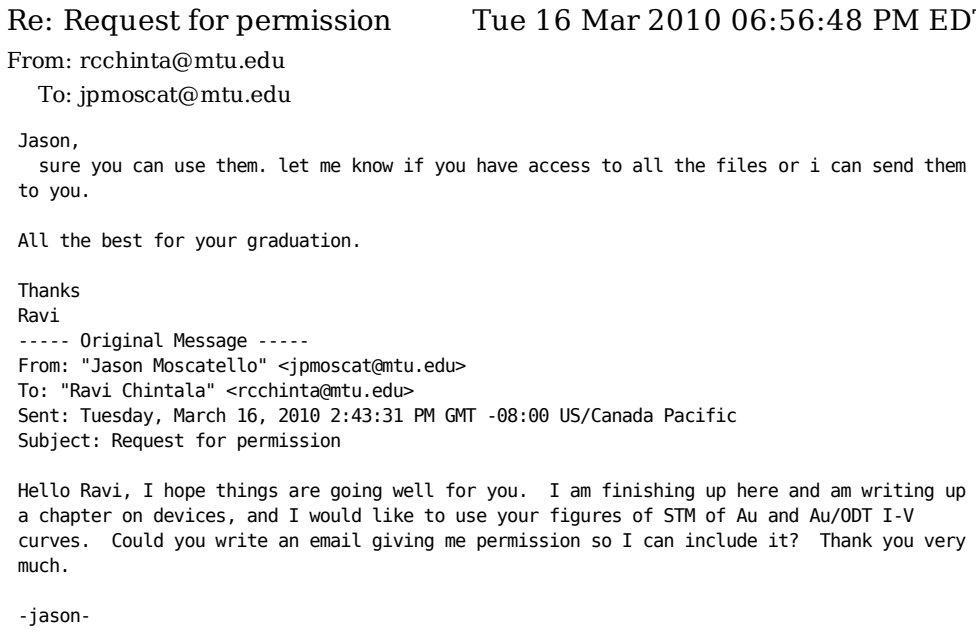




\section{C.11 Shun Wu. Reference [48]}

To whom it concerns,

This is a formal assertion for my former labmate Jason Moscatello, who currently works in Dr. Yoke Khin Yap's laboratory at Michigan Tech University. As per Jason's request, I am allowing him to cite any of my previous results (May. 2006 - Dec. 2007) or pictures / diagrams that have been published in my Master report, "Growth and characterization of carbon nanotubes and quantum dots", for the purpose of his Ph.D. dissertation and oral presentation. If you have any questions, please contact me at:

Shun $\mathrm{Wu}$

116 Cardwell Hall

Kansas State University

Manhattan, KS, USA

66506-2601

Email: wshun@phys.ksu.edu

Lab phone: 785-532-1645

Cellphone: 785-236-0743

Regards,

Signature:

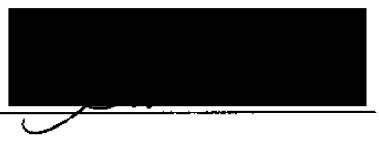
Date: $\quad 10 / 09 / 2009$ 


\title{
C.12 Springer, Reference [176]
}

\author{
RE: Customer question from \\ springer.com \\ From: Permissions.Dordrecht@springer.com \\ To: jpmoscat@mtu.edu \\ Sender: Nel.vanderWerf@springer.com
}

Mon 18 Jan 2010 06:02:06 AM

Dear Sir,

With reference to your request (copy herewith) to reprint material on which Springer Science and Business Media controls the copyright, our permission is granted, free of charge, for the use indicated in your enquiry.

This permission

- allows you non-exclusive reproduction rights throughout the World.

permission includes use in an electronic form, provided that content is

* password protected;

* at intranet;

- excludes use in any other electronic form. Should you have a specific project in mind, please reapply for permission.

requires a full credit (Springer/Kluwer Academic Publishers book/journal title, volume, year of publication, page, chapter/article title, name(s) of author(s), figure number $(s)$, original copyright notice) to the publication in which the material was originally published, by adding: with kind permission of Springer Science and Business Media. The material can only be used for the purpose of defending your dissertation, and with a maximum of 40 extra copies in paper.

Permission free of charge on this occasion does not prejudice any rights we might have to charge for reproduction of our copyrighted material in the future.

Best regards,

Nel van der Werf (Ms)

Rights and Permissions/Springer

Van Godewijckstraat 30 | P.0. Box 17

3300 AA Dordrecht | The Netherlands

tel $+31(0) 786576298$

fax $+31(0) 786576-300$

Nel.vanderwerf aspringer.com

unw. springer. com

...--Original Message-....

From: SpringerAlerts@springeronline.com [mailto:SpringerAlerts@springeronline.com]

Sent: vrijdag 15 januari $20105: 32$

To: Permissions Europe/NL 
Subject: Customer question from springer.com

Springer Representative:

I would like to request permission to use three figures from one of your publications. All the details are listed below; if you need more information, please just ask. Thank you for your time and assistance.

Sincerely,

Jason Moscatello

My Information

Jason P Moscatello

118 Fisher Hall

1400 Townsend Drive

Houghton, MI

fax: $906-487-2933$

email: jpmoscat@mtu.edu

Book

ISBN: $0-306-47292-9$

Title: Scanning Electron Microscopy and X-Ray Microanalysis

Authors: Joseph Goldstein, Dale Newbury, David Joy, Charles Lyman, Patrick Echlin, Eric Lifshin, Linda Sawyer and Joseph Michael

Publication: 2003

Intent

I would like to use Figure 1.3 (page 4) and Figure $2.2 \&$ Figure 2.3 (page 23). The figures will be used as cited figures in the equipment chapter of my physics Ph.D. dissertation.

This dissertation will be published internally at Michigan Technological University and is not for profit.

sender name: Jason Moscatello

sender email: jpmoscat@mtu.edu 
https://huskymail.mtu.edu/zimbra/

INTERNAL NAME: Rights and Permissions Department - Springer Netherlands ORIGINAL URL: http://Www. springer. com/rights?SGWID=0-122-19-161418- $\Theta$

$$
\text { ] }
$$




\title{
C.13 University of Cambridge, Reference [33]
}

\author{
Zimbra: jpmoscat@mtu.edu \\ https://huskymail.mtu.edu/zimbra/ \\ Zimbra Collaboration Suite \\ jpmoscat@mtu.edu

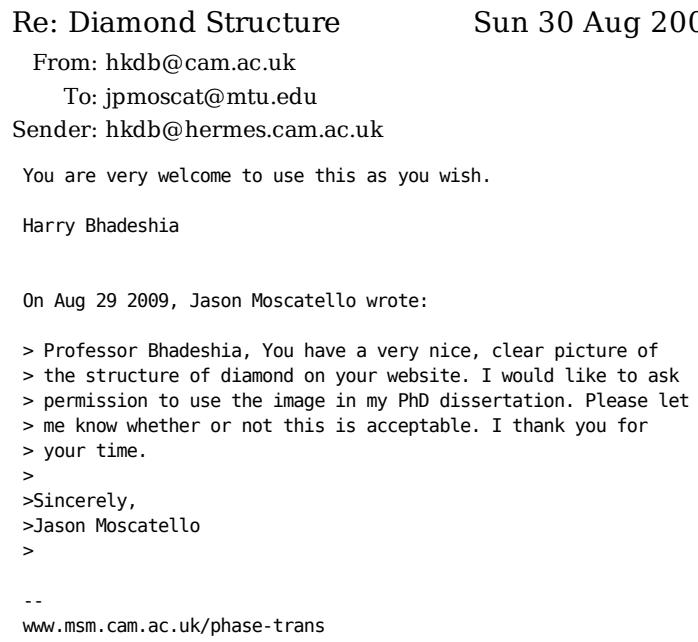




\section{Bibliography}

[1] R. Lloyd Carroll and Christopher B. Gorman. The genesis of molecular electronics. Angewandte Chemie International Edition, 41(23):4378-4400, 2002.

[2] M.T. Bohr, R.S. Chau, T. Ghani, and K. Mistry. The high-k solution. IEEE Spectrum, October 2007. NA.

[3] C.P. Collier, G. Mattersteig, E.W. Wong, Y. Luo, K. Beverly, J. Sampaio, F.M. Raymo, J.F. Stoddart, and J.R. Heath. A [1]catenane-based solid state electronically reconfigurable switch. Science, 289(5482):1172-1175, 2000.

[4] Yi Luo, C. Patrick Collier, Jan O. Jeppensen, Kent A. Nielsen, Erica Delonno, Greg Ho, Julie Perkins, Hsian-Rong Tseng, Tohru Yamamota, J. Fraser Stoddart, and James R. Heath. Two-dimensional molecular electronics circuits. Chem. Phys. Chem., 3:519-525, 2002.

[5] A.O. Solak, S. Ranganathan, T. Itoh, and R.L. McCreery. Mechanism for conductance switching in carbon-based molecular electronic junctions. Electrochem. Solid-State Lett., 5:E43, 2002.

[6] Robert M. Metzger, Tao Xu, and Ian R. Peterson. Electrical rectification by a monolayer of hexadecylquinolinium tricyanoquinodimethanide measured between macroscopic gold electrodes. J. Phys. Chem. B, 105(30):7280, 2001.

[7] Richard L. McCreery, Jon Dieringer, Ali Osman Solak, Brian Snyder, Aletha M. Nowak, William R. McGovern, and Stacy DuVall. Molecular rectification and conductance switching in carbon-based molecular junctions by structural rearrangement accompanying electron injection. J. Am. Chem. Soc., 125:10748-10758, 2003. 
[8] J. Chen, M.A. Reed, A.M. Rawlett, and J.M. Tour. Large on-off ratios and negative differential resistance in a molecular electronic device. Science, 286(5444):1550-1552, 1999.

[9] J.E. Green, J.W. Choi, A. Boukai, Y. Bunimovich, E. Johnston-Halperin, E. Delonno, Y. Luo, B.A. Sheriff, K. Xu, Y.S. Shin, H.-R. Tseng, J.F. Stoddart, and J.R. Heath. A 160-kilobit molecular electronic memory patterned at $10^{11}$ bits per square centimetre. Nature, 445:414$417,2007$.

[10] Richard L. McCreery. Molecular electronic junctions. Chem. Mater., 16(23):4477-4496, 2004.

[11] Sumio Iijima. Helical microtubules of graphitic carbon. Nature(London), 354:56, 1991.

[12] M.S. Dresselhaus and G. Dresselhaus, editors. Carbon Nanotubes: Synthesis, Structure, Properties and Applications. Springer-Verlag, Berlin, Germany, 2001.

[13] Peter J. F. Harris. Carbon Nanotubes and Related Structures: New Materials for the Twentyfirst Century. Cambridge University Press, 2003.

[14] Hong Li, Qing Zhang, and Jingqi Li. Carbon-nanotube-based single-electron/hole transistors. Appl. Phys. Lett., 88:013508, 2006.

[15] Ali Javey, Damon B. Farmer Jing Guo, Qian Wang, Roy G. Gordon, Mark Lundstrom, and Hongjie Dai. Carbon nanotube field-effect transistors with integrated ohmic contacts and high- $\kappa$ gate dielectrics. Nanolett, 4(3):447-450, 2004.

[16] R. Martel, T. Schmidt, H.R. Shea, T. Hertel, and Ph. Avourisa. Single- and multi-wall carbon nanotube field-effect transistors. Appl. Phys. Lett., 73(17):2447-2449, 1998.

[17] S.J. Wind, J. Appenzeller, R. Martel, V. Derycke, and Ph. Avouris. Vertical scaling of carbon nanotube field-effect transistors using top gate electrodes. Appl. Phys. Lett., 80(20):3817-3819, 2002.

[18] Sander J. Tans, Alwin R.M. Verschueren, and Cees Dekker. Room-temperature transistor based on a single carbon nanotubes. Nature, 393:49-52, 1998. 
[19] Jing Kong, Nathan R. Franklin, Chongwu Zhou, Michael G. Chapline, Shu Peng, Kyeongjae Chou, and Hongjie Dai. Nanotube molecular wires as chemical sensors. Science, 287:622-625, 2000.

[20] Philip G. Collins, Keith Bradley, Masa Ishigami, and A. Zettl. Extreme oxygen sensitivity of electronic properties of carbon nanotubes. Science, 287(5459):1801, 2000.

[21] Hongjie Dai, Jason H. Hafner, Andrew G. Rinzler, Daniel T. Coilbert, and Richard E. Smalley. Nanotubes as nanoprobes in scanning probe microscopy. Nature, 384:147-150, 1996.

[22] E.S. Snow, P.M. Campbell, and J.P. Novak. Single-wall carbon nanotube atomic force microscope probes. Appl. Phys. Lett., 80(11):2002-2004, 2002.

[23] Jason H. Hafner, Chin Li Cheung, and Charles M. Lieber. Growth of nanotubes for probe microscopy tips. Nature, 398:761-762, 1999.

[24] Benjaming Ulmen, Vijaya K. Kayastha, Adam DeConinck, Jiesheng Wang, and Yoke Khin Yap. Stability of field emission current from various types of carbon nanotube films. Diamond E Related Materials, 15:212-216, 2006.

[25] F. Kreupl, A.P. Graham, G.D. Duesberg, W. Steinhögl, M. Liebau, E. Unger, and W. Hölein. Carbon nanotubes in interconnect applications. Microelecronic Engineering, 64:399, 2002.

[26] Jun Li, Qi Ye, Alan Cassell, Hou Tee Ng, Ramsey Stevens, Jie Han, and M. Meyyappan. Bottom-up approach for carbon nanotube interconnects. Appl. Phys. Lett., 82(15), 2003.

[27] Alberto Bianco, Kostas Kostarelos, and Maurizio Prato. Applications of carbon nanotubes in drug delivery. Current Opinion in Chemical Biology, 9:674, 2005.

[28] Yuehe Lin, Fang Lu, Yi Tu, and Zhifeng Ren. Glucose biosensors based on carbon nanotube nanoelectrode ensembles. Nano Letters, 4(2):191-195, 2004.

[29] Jun Li, Hou Tee Ng, Alan Cassell, Wendy Fan, Hua Chen, Qi Ye, Jessica Koehne, Jie Han, and M. Meyyappan. Carbon nanotube nanoelectrode array for ultrasensitive DNA detection. Nano Letters, 3(5):597-602, 2003. 
[30] Herbert Meislich, Howard Nechamkin, Jacob Sharefkin, and George J. Hademenos. Schaum's Outlines of Theory and Problems of Organic Chemistry. McGraw-Hill, third edition, 1999.

[31] R. Saito, G. Dresselhaus, and M.S. Dresselhaus. Physical Properties of Carbon Nanotubes. Imperial College Press, 1998.

[32] François Léonard. The Physics of Carbon Nanotube Devices. Micro \& Nano Technologies. William Andrew Inc., 2008.

[33] H.K.D.H. Bhadeshia. Some crystal structures. University of Cambridge, Phase Transformations \& Complex Properties Research Group Website, August 2009.

[34] Sumio Iijima and Toshinari Ichihashi. Single-shell carbon nanotubes of 1-nm diameter. Nature, 363:603, 1993.

[35] M.P. Anatram and F. Léonard. Physics of carbon nanotube electronic devices. Rep. Prog. Phys., 69:507, 2006.

[36] Yury Gogotsi, editor. Nanotubes and Nanofibers. CRC Press and the Taylor \& Francis Group, 2006.

[37] J. Emsley. The Elements. Oxford University Press, 1991.

[38] I.S. Kulikov, editor. Thermodynamics of Carbides and Nitrides. Metallurgiya, 1988. Russian.

[39] H. Yokoyama, Numakura H, and M Koiwa. The solubility and diffusion of carbon in palladium. Acta Metallurgica et Materialia, 46:2823, 1998.

[40] I.S. Kulikov and Termodinamika Oskidov, editors. Thermodynamics of Oxides. Metallurgiya, 1986.

[41] Randall M. German. Sintering Theory and Practice. Wiley-Interscience, 1996.

[42] Young Joon Yoon, Jun Cheol Bae, Hong Koo Baik, Seong Jin Cho, Se-Jong Lee, Kie Moon Song, and No Seung Myung. Nucleation and growth control of carbon nanotubes in CVD process. Physica B, 323:318, 2002. 
[43] V.K. Kayastha, Y.K. Yap, S. Dimovski, and Y. Gogotsi. Controlling dissociative adsorption for effective growth of carbon nanotubes. Appl. Phys. Lett., 85:3265, 2004.

[44] V.K. Kayastha, Y.K. Yap, Z. Pan, I.N. Ivanov, A.A. Puretzky, and D.B. Geohegan. Highdensity vertically aligned multiwalled carbon nanotubes with tubular structures. Appl. Phys. Lett., 86:253105, 2005.

[45] Yiming Li, Woong Kim, Yuegang Zhang, Marco Rolandi, Dunwei Wang, and Hongjie Dai. Growth of single-walled carbon nanotubes from discrete catalytic nanoparticles of various sizes. J. Phys. Chem. B, 105(46):11424, 2001.

[46] Hee Cheul Choi, Woong Kim, Dunwei Wang, and Hongjie Dai. Delivery of catalytic metal species onto surfaces with dendrimer carriers for the synthesis of carbon nanotubes with narrow diameter distribution. J. Phys. Chem. B, 106(48):12361, 2002.

[47] Woong Kim, Hee Cheul Choi, Moonsub Shim, Yiming Li, Dunwei Wang, and Hongjie Dai. Synthesis of ultralong and high percentage of semiconducting single-walled carbon nanotubes. Nano Lett., 2(7):703, 2002.

[48] Shun Wu. Growth and characterization of carbon nanotubes and quantum dots. Master's thesis, Michigan Technological University, 2007.

[49] Douglas B. Chrisey and Graham K. Hubler, editors. Pulsed Laser Deposition of Thin Films. John Wiley \& Sons, Inc., 1994.

[50] Yoke Khin Yap. Deep-UV Generation by CLBO Crystal and its Utilization for $C N$ and c-BN Thin Film Deposition. PhD thesis, Osaka University, 1999.

[51] G.B. Sergeev. Nanochemistry. Elsevier, 2006.

[52] Anna Moisala, Albert G. Nasibulin, and Esko I. Kauppinen. The role of metal nanoparticles in the catalytic production of single-walled carbon nanotubes - a review. J. Phys.: Condens. Matter, 15:S3011, 2003.

[53] Z.W. Pan, S.S. Xie, B.H. Chang, C.Y. Wang, L. Lu, W. Liu, W.Y. Zhou, W.Z. Li, and L.X. Qian. Very long carbon nanotubes. Nature, 394:631, 1998. 
[54] Shoushan Fan, Michael G. Chapline, Nathan R. Franklin, Thomas W. Tombler, and Alan M. Cassell. Self-oriented regular arrays of carbon nanotubes and their field emission properties. Science, 283(5401):512, 1999.

[55] Guangyu Zhang, David Mann, Li Zhang, Ali Javey, Yiming Li, Erhan Yenilmez, Qian Wang, James P. McVittie, Yoshio Nishi, James Gibbons, and Hongjie Dai. Ultra-high-yield growth of vertical single-walled carbon nanotubes: Hidden roles of hydrogen and oxygen. PNAS, 102:16141, 2005.

[56] Alan M. Cassell, Jeffrey A. Raymakers, Jing Kong, and Hongjie Dai. Large scale CVD synthesis of single-walled carbon nanotubes. J. Phys. Chem. B, 103:6484, 1999.

[57] Avertik R. Harutyunyan, Bhabendra K. Pradhan, U.J. Kim, Gugang Chen, and P.C. Eklund. CVD synthesis of single wall carbon nanotubes under "soft" conditions. Nano. Lett., 2:525, 2002 .

[58] Takayuki Iwasaki, Goufang Zhong, Takumi Aikawa, Tsuyoshi Yoshida, and Hiroshi Kawarada. Direct evidence for root growth of vertically aligned single-walled carbon nanotubes by microwave plasma chemical vapor deposition. J. Phys. Chem. B, 109:19556, 2005.

[59] Hongjie Dai, Andrew G. Rinzier, Pasha Nikolaev, Andreas Thess, Daniel T. Colbert, and Richard E. Smalley. Single-wall nanotubes produced by metal-catalyzed disproportionation of carbon monoxide. Chemical Physics Letters, 260:471, 1996.

[60] W.E. Alvarez, A. Borgna, and D.E. Resasco. Synergism of Co and Mo in the catalytic production of single-wall carbon nanotubes by decomposition of CO. Carbon, 29:547, 2001.

[61] Yoichi Murakami, Shohei Chiashi, Yuhei Miyauchi, Minghui Hu, Masaru Ogura, Tatsuya Okubo, and Shigeo Maruyama. Growth of vertically aligned single-walled carbon nanotube filme on quartz substrates and their optical anisotropy. Chem. Phys. Lett., 385:298, 2004.

[62] Husnu Emrah Unalan and Manish Chhowalla. Investigation of single-walled carbon nanotube growth parameters using alcohol catalytic chemical vapour depostion. Nanotech., 16:2153, 2005. 
[63] R.S. Wagner and W.C. Ellis. Vapor-liquid-solid mechansim of single crystal growth. Appl. Phys. Lett., 4(5):89, 1964.

[64] E.I. Givargizov. Current Topics in Materials Science, volume I. North Holland, 1978.

[65] Gun-Do Lee, Seungwu Han, Jaejun Yu, and Jisoon Ihm. Catalytic decomposition of acetylene Fe(001): A first-principles study. Phys. Rev. B, 66:081403(R), 2002.

[66] V. Samardzic. Fundamentals of metal alloys, equilibrium diagrams. Website. http://web.njit.edu/ vxs9076/me215/ME215-4CMP.ppt, visited 9-19-09.

[67] Jason P. Moscatello, Jiesheng Wang, Benjamin Ulmen, Samuel L. Mensah, Ming Xie, Shun Wu, Archana Pandey, Chee Huei Lee, Abhishek Prasad, Vijaya K. Kayastha, and Yoke Khin Yap. Controlled growth of carbon, boron nitride and zinc oxide nanotubes. IEEE Sensors Journal, 8:922, 2008. Special Issue on Nanosensors for Defense \& Security.

[68] Brian W. Smith and David E. Luzzi. Electron irradiation effects in single wall carbon nanotubes. J. Appl. Phys., 90:3509, 2001.

[69] A. V. Krasheninnikov, F. Banhart, J. X. Li, A. S. Foster, and R. M. Nieminen. Stability of carbon nanotubes under electron irradiation: Role of tube diameter and chirality. Phys. Rev. B, 72(12):125428, Sep 2005.

[70] C. Gomez-Navarro, P.J. De Pablo, J. Gomez-Herrero, B. Biel, F.J. Garcia-Vidal, A. Rubio, and F. Flores. Tuning the conductance of single-walled carbon nanotubes by ion irradiation in the anderson localization regime. Nature Materials, 4:534, 2005.

[71] M.S. Raghuveer, A. Kumar, M.J. Frederick, G.P. Louie, P.G. Ganesan, and G. Ramanath. Site-selective functionalization of carbon nanotubes. Advanced Materials, 18:547, 2006.

[72] Sebastian Osswald, Mickael Havel, and Yury Gogotsi. Monitoring oxidation of multiwalled carbon nanotubes by raman spectroscopy. J. Raman Spectrosc., 38:728, 2007.

[73] M. Vesali Naseh, A.A. Khodadadi, Y. Mortazavi, O. Alizadeh Sahraei, F. Pourfayaz, and S. Mosadegh Sedghi. Functionalization of carbon nanotubes using nitric acid oxidation and DBD plasma. World Academy of Science, Engineering and Technology, 49:177, 2009. 
[74] Cheol Jin Lee, Jung Hoon Park, and Jeunghee Park. Synthesis of bamboo-shaped multiwalled carbon nanotubes using thermal chemical vapor deposition. Chem. Phys. Lett., 323:560, 2000.

[75] Z.Y. Yuang, I.P. Chien, J.F. Lai, T.S. Lai, and C.H. Tsai. The effects of ammonia on the growth of large-scale patterned aligned carbon nanotubes using thermal chemical vapor deposition method. Diamond and Related Materials, 13:1203, 2004.

[76] Cheol Jin Lee and Jeunghee Park. Growth model of bamboo-shaped carbon nanotubes by thermal cehmical vapor deposition. Appl. Phys. Lett., 77(21):3397, 2000.

[77] Jae Won Jang, Cheol Eui Lee, Seung Chul Lyu, Tae Jae Lee, and Cheol Jin Lee. Structural study of nitrogen-doping effects in bamboo-shaped multiwalled carbon nanotubes. Appl. Phys. Lett., 84(15):2877-2879, 2004.

[78] Kyoung-Yong Chun, Heon Sang Lee, and Cheol Jin Lee. Nitrogen doping effects on the structure behavior and the field emission performance of double-walled carbon nanotubes. Carbon, 47(1):169-177, 2009.

[79] M. Meyyappan, Lance Delzeit, Alan Cassell, and David Hash. Carbon nanotube growth by PECVD: a review. Plasma Sources Sci. Technol., 12(205), 2003.

[80] M. Chowalla, K.B.K Teo, C. Ducati, N.L. Rupesinghe, G.A.J. Amaratunga, A.C. Ferrari, D. Roy, J. Roberston, and W.I. Milne. Growth process conditions of vertically aligned carbon nanotubes using plasma enhanced chemical vapor deposition. Journal of Applied Physics, 90(10), 2001.

[81] Benjamin Ulmen. Growth of vertically-aligned carbon nanotubes and their application as electron field emitters. Master's thesis, Michigan Technological University, 2007.

[82] Chengghuo Hu, Chunhai Yang, and Shengshui Hu. Hydrophobic adsorption of srufactants on water-soluble carbon nanotubes: A simple approach to improve sensitivity and anitfouling capacity of carbon nanotubes-based electrochemical sensors. Electrochemistry Communications, 9:128, 2007.

[83] A. M. Rao, J. Chen, E. Richter, U. Schlecht, P. C. Eklund, R. C. Haddon, U. D. Venkateswaran, 
Y.-K. Kwon, and D. Tománek. Effect of van der waals interactions on the raman modes in single walled carbon nanotubes. Phys. Rev. Lett., 86(17):3895-3898, Apr 2001.

[84] Francisco Pompeo and Daniel E. Resasco. Water solubilization of single-walled carbon nanotubes by functionalization with glucosamine. Nano. Lett., 2(4):369, 2002.

[85] Jeffrey L. Bahr and James M. Tour. Covalent chemistry of single-wall carbon nanotubes. J. Mater. Chem., 12:1952, 2002.

[86] Michael J. O'Connell, Peter Boul, Lars M. Ericson, Chad Huffman, Yuhuang Wang, Erik Haroz, Cynthia Kuper, Jim Tour, Kevin D. Ausman, and Richard E. Smalley. Reversible water-solubilization of single-walled carbon nanotubes by polymer wrapping. Chem. Phys. Lett., 342:265-271, 2001.

[87] Valerie C. Moore, Michael S. Strano, Erik H. Haroz, Robert H. Hauger, and Richard E. Smalley. Individually suspended single-walled carbon nanotubes in various surfactants. Nano Lett., 3(10):1379-1382, 2003.

[88] Kin tak Lau, Mei Lu, Chun ki Lam, Hoi yan Cheung, Fen-Lin Sheng, and Hu-Lin Li. Thermal and mechanical properties of single-walled carbon nanotube bundle-reinforced epoxy nanocomposites: the role of solvent for nanotube dispersion. Composites Science and Technology, 65(5):719-725, 2005.

[89] G. U. Sumanasekera, B. K. Pradhan, H. E. Romero, K. W. Adu, and P. C. Eklund. Giant thermopower effects from molecular physisorption on carbon nanotubes. Phys. Rev. Lett., 89(16):166801, Sep 2002.

[90] David A. Britz and Andrei N. Khlobystov. Noncovalent interactions of molecules with single walled carbon nanotubes. Chem. Soc. Rev., 35:637-659, 2006.

[91] Yi Lin, Shelby Taylor, Huaping Li, K.A. Shiral Fernando, Liangwei Qu, Wei Wang, Lingrong Gu, Bing Zhou, and Ya-Ping Sun. Advances toward bioapplications of carbon nanotubes. J. Mater. Chem., 14:527-541, 2004.

[92] Michael J. O'Connell, Sergei M. Bachilo, Chad B. Huffman, Valerie C. Moore, Michael S. Strano, Erik H. Haroz, Kristy L. Rialon, Peter J. Boul, William H. Noon, Carter Kittrell, 
Jianpeng Ma, Robert H. Hauge, R. Bruce Weisman, and Richard E. Smalley. Band gap fluorescence from individual single-walled carbon nanotubes. Science, 297(5581):593-596, 2002.

[93] A. Bachtold, P.Hadley, T. Nakanishi, and C. Dekker. Logic circuits with carbon nanotube transistors. Science, 294:1317-1320, 2001.

[94] A. Javey, M. Shim, and H. Dai. Electrical properties and devices of large-diameter single-walled carbon nanotubes. Appl. Phys. Lett., 80:1064, 2002.

[95] X. Liu, C. Lee, C. Zhou, and J. Han. Carbon nanotube field-effect inverters. Appl. Phys. Lett., 79:3329, 2001.

[96] P.J. Burke. Encyclopedia of Nanoscience and Nanotechnology, volume X, chapter Nanodielectrophoresis: Electronic Nanotweezers, pages 1 - 19. American Scientific Publishers, 2003.

[97] Thomas B. Jones. Electromechanics of Particles. Cambridge University Press, 1995.

[98] Manuel J. Mendes, Howard K. Schmidt, and Matteo Pasquali. Brownian dynamics simulations of single-wall carbon nanotube separation by type using dielectrophoresis. J. Phys. Chem. B, 112:7467-7477, 2008.

[99] C.F. Bohren and D.R. Huffman. Absorption and Scattering and Scattering of Light by Small Particles. Wiley-VCH, New York, 1983.

[100] Ji-Eun Kim and Chang-Soo Han. Use of dielectrophoresis in the fabrication of an atomic force microscope tip with a carbon nnaotube: a numerical analysis. Nanotechnology, 16:2245-2250, 2005.

[101] A. Ugawa, A.G. Rinzler, and D.B. Tanner. Far-infrared gaps in single-wall carbon nanotubes. Phys. Rev. B: Condens. Mater. Phs., 60:R11305-R11308, 1999.

[102] Vijaya K. Kayastha. Catalytic Growth of Single-, Double-, and Multi-walled Carbon Nanotubes and Studies of their Potential Applications. PhD thesis, Michigan Technological University, 2006.

[103] K. Yamamoto, S. Akita, and Y. Nakayama. Carbon nanotube tips for a scanning probe microscope: their fabrication and properties. J. Phys. D: Appl. Phys., 31:L34, 1998. 
[104] Libao An. Real-time electrical characterization of carbon nanotube deposition onto electrode gaps by dielectrophoresis. PhD thesis, Michigan Technological University, 2007.

[105] Jason Moscatello, Vijaya Kayastha, Archana Pandey, Benjamin Ulmen, and Yoke Khin Yap. Dielectrophoretic deposition of carbon nanotubes with controllable density and alignment. Mater. Res. Soc. Symp. Proc, 1057, 2008. Paper 1057-II15-07.

[106] J. Menda, B. Ulmen, L.K. Vanga, V.K. Kayastha and. Y.K Yap, Z. Pan, I.N. Ivanov, A.A. Puretzky, and D.B. Geohegan. Structural control of vertically aligned multiwalled carbon nanotubes by radio-frequency plasmas. Appl. Phys. Lett., 87:173106, 2005.

[107] M. Dimaki and P. Bøggild. Dielectrophoresis of carbon nanotubes using microelectrodes: a numerial study. Nanotechnology, 15:1095, 2004.

[108] Landolt-Börnstein. IV/17 Static Dielectric Constants of Pure Liquids and Binary Liquid Mixtures. Springer Berlin, Heidelberg, 2008.

[109] Rosa H M Chan, Carmen K M Fung, and Wen J Li. Rapid assembly of carbon nanotubes for nanosensing by dielectrophoretic force. Nanotechnology, 15:S672-S677, 2004.

[110] J. A. Dean. Lange's Handbook of Chemistry. McGraw Hill, 14 edition, 1992.

[111] Martial Duchamp, Kyumin Lee, Benjamin Dwir, Jin Won Seo, Eli Kapon, László Forró, and Arnaud Magrez. Controlled positioning of carbon nanotubes by dielectrophoresis: insights into the solvent and substrate role. ACS Nano, 2010. DOI: 10.1021/nn901559q, Published online January 7th, 2010.

[112] Allen J. Bard and Larry R. Faulkner. Electrochemical Methods: Fundamentals and Applications. John Wiley \& Sons, Inc., 2001.

[113] A. Ramos, H. Morgan, N.G. Green, and A. Castellanos. Ac electrokinetics: a review of forces in microelectrode structures. J. Phys. D: Appl. Phys., 31:2338, 1998.

[114] H.P. Schwan. Electrode polarization impedance and measurements in biological materials. Ann. New York Acad. Sci., 148:191, 1968. 
[115] Walt A. de Heer, A. Châtelain, and D. Ugarte. A carbon nanotube field-emission electron source. Science, 270(5239):1179-1180, 1995.

[116] W.B. Choi, D.S. Chung, J.H. Kang, H.Y. Kim, Y.W. Jin, I.T. Han, Y.H. Lee, J.E. Jung, N.S. Lee, G.S. Park, and J.M. Kim. Fully selaed, high brightness carbon-nanotube field-emission display. Applied Physics Letters, 75(20):3129-3131, 1999.

[117] Niels de Jonge, Yann Lamy, Koen Schoots, and Tjerk H. Oosterkamp. High brightness electron beam from a multi-walled carbon nanotube. Nature, 420:393, 2002.

[118] G.Z. Yue, Q. Qiu, Bo Gao, Y. Cheng, J. Zhang, H. Shimoda, S. Chang, J.P. Lu, and O. Zhou. Generation of continuous and pulsed diagnostic imaging x-ray radiation using a carbon-nanotube-based field-emission cathode. Applied Physics Letters, 81:355, 2002.

[119] Archana Pandey, Abhishek Prasad, Jason Moscatello, Benjamin Ulmen, and Yoke Khin Yap. Enhanced field emission stability and density produced by conical bundles of catalyst-free carbon nanotubes. Carbon, 48:287-292, 2010.

[120] Jie Liu, Andrew G. Rinzler, Hongjie Dai, Jason H. Hafner, R. Kelley Bradley, Peter J. Boul, Adrian Lu, Terry Iverson, Konstantin Shelimov, Chad B. Huffman, Fernando RodriguezMacias, Young-Seok Shon, T. Randall Lee, Daniel T. Colbert, and Richard E. Smalley. Fullerene pipes. Science, 280(5367):1253-1256, 1998.

[121] Jin Zhang, Hongling Zou, Quan Qing, Yanlian Yang, Qingwen Li, Zhongfan Liu, Xinyon Guo, and Zuliang Du. Effects of chemical oxidation on the structure of single-walled carbon nanotubes. Journal of Physical Chemistry B, 107(16):3712-3718, 2003.

[122] Centers for Disease Control and U.S. Department of Health Prevention. National diabetes fact sheet, 2007.

[123] Yuehe Lin, Wassana Yantasee, and Joseph Wang. Carbon nanotubes (CNTs) for the development of electrochemical biosensors. Frontiers in Bioscience, 10:492-505, 2005.

[124] Joseph Wang. Carbon-nanotube based electrochemical biosensors: a review. Electroanalysis, $17: 7-14,2004$. 
[125] Xinyu Pand, Dongmei He, Shenglian Luo, and Qingyuan Cai. An amperometric glucose biosensor fabricated with $\mathrm{Pt}$ nanoparticle-decorated carbon nanotubes $/ \mathrm{TiO}_{2}$ nanotube arrays composite. Sensors and Actuators: B. Chemical, 137:134-138, 2009.

[126] Briza Pérez, Martin Pumera, Manel del Valle, Arben Merkoçi, and Salvador Alegret. Glucose biosensor based on carbon nanotube epoxy composites. Sensors and Actuators, 137:1694-1698, 2009.

[127] Hao Tang, Jinhua Chen, Shouzhuo Yao, Lihua Nie, Guohon Deng, and Yafei Kuang. Amperometric glucose biosensor based on adsorption of glucose oxidase at platinum nanoparticlemodified carbon nanotube electrode. Analytical Biochemistry, 331(1):89-97, 2004.

[128] Jinyan Jia, Wenjun Guan, Minghao Sim, Yongquan Li, and Hong Li. Carbon nanotubes based glucose needle-type biosensor. Sensors, 8(3):1712-1718, 2008.

[129] D. Müller. Biochemistry, 199:136, 1928.

[130] D. Keilin and E.F. Hartree. Specificity of glucose oxidase (notatin). Biochem. J., 50(3):331, 1952.

[131] Julio Raba and Horacio A. Mottola. Glucose oxidase as an analytical reagent. Critical Reviews in Analytical Chemistry, 25(1):1-42, 1995.

[132] Ziyi Wang, Shuna Liu, Ping Wu, and Chenxin Cai. Detection of glucose based on direct electron transfer reaction of glucose oxidase immobilized on highly ordered polyaniline nanotubes. Analytical Chemistry, 81(4):1638-1645, 2009.

[133] James M. Tour, William A. Reinerth, Leroy Jonesii, Timothy P. Burgin, Chong-Wu Zhou, C.J. Muller, M.R. Deshpande, and Mark A. Reed. Recent advances in molecular scale electronics. Annals of the New York Academy of Sciences, 852:197-204, 2006. Issue: Molecular Electronics: Science and Technology.

[134] Noel S. Hush. An overview of the first half-century of molecular electronics. Annals of the New York Academy of Sciences, 1006(1):1-20, 2003.

[135] M.A. Reed, C. Zhou, C.J. Muller, T.P. Burgin, and J.M. Tour. Conductance of a molecular junction. Science, 278(5336):252-254, 1997. 
[136] J. Chen, M.A. Reed, A.M. Rawlett, and J.M. Tour. Large on-off ratios and negative differential resistance in a molecular electronic device. Science, 286(5444):1550-1552, 1999.

[137] Robert M. Metzger, Bo Chen, Ulf Hoepner, M.V. Lakshmikantham, Dominique Vuillaume, Tsuyoshi Kawai, Xiangli Wu, Hioaki Tachibana, Terry V. Hughes, Hiromi Sakurai, Jeffery W. Baldwin, Christina Hosch, Michael P. Cava, Ludwig Brehmer, and Geoffrey J. Ashwell. Unimolecular electrical rectification in hexadecylquinolinium tricyanoquinodimethanide. Journal of the Americal Chemical Society, 119(43):10455-10466, 1997.

[138] C. Joachim and J.K. Gimzewski. An electromechanical amplifier using a single molecule. Chemical Physics Letters, 265(3-5):353-357, 1997.

[139] Hemk W. Ch. Postma, Tijs Teepen, Zhen Yao, Milena Grifoni, and Cees Dekker. Carbon nanotube single-electron transistors at room temperature. Science, 293(5527):76-79, 2001.

[140] Jiwoong Park, Abhay N. Pasupathy, Jonas I. Goldsmith, Connie Chang, Yuval Yaish, Jason R. Petta, Marie Rinkoski, James P. Sethna, Héctor D. Abruña, Paul L. McEuen, and Daniel C. Ralph. Coulomb blockade and the Kondo effect in single-atom transistors. Nature, 417:722$725,2002$.

[141] V. Derycke, R. Martel, J. Appenzeller, and Ph. Avouris. Carbon nanotube inter- and intramolecular logic gates. Nano Letters, 1(9):453-456, 2001.

[142] Yu Huang, Xiangfeng Duan, Yi Cui, Lincoln J. Lauhon, Kyoung-Ha Kim, and Charles M. Lieber. Logic gates and computation from assembled nanowire building blocks. Science, 294(5545):1313-1317, 2001.

[143] Adrian Bachtold, Peter Hadley, Takeshi Nakanishi, and Cees Dekker. Logic circuits with carbon nanotube transistors. Science, 294(5545):1317-1320, 2001.

[144] Robert F. Service. Can chemists assemble a future for molecular electronics? Science, 295(5564):2398-2399, 2002.

[145] Johan Åkerman, Philip Brown, Don Gajewski, Mark Griswold, Jason Janesky, Matthew Martic, Hamere Mekonnen, Joseph J. Nahas, Srinivas Pietambaram, J.M. Slaughter, and Saied 
Tehrani. Reliability of 4mbit MRAM. IEEE $43^{\text {rd }}$ Annual International Reliability Physics Sypmosium, pages 163-167, 2005.

[146] MRAM technology attributes. Website, February 2010. http://everspin.com/technology.html.

[147] B.N. Engel, J. Åkerman, B. Butcher, R.W. Dave, M. DeHerrera, M. Durlam, G. Grynkewich, S.V. Pietambaram J. Janesky, N.D. Rizzo, J.M. Slaughter, K. Smith, J.J. Sun, and S. Tehrani. A 4-Mb toggle MRAM based on a novel bit and switching method. IEEE Transactions on Magnetics, 41(1), 2005.

[148] Mark LePedus. Toshiba rolls high-speed, 128 Mbit FeRAM. Website, February 2009. http://www.eetimes.com/conf/isscc/showArticle.jhtml?articleID=213400572\&kc=3681.

[149] Ferroelectric Random Access Memories, volume 93/2004 of Topics in Applied Physics, chapter The Current Status of FeRAM, pages 138-148. Springer Berlin, Heidelberg, 2004.

[150] F. Pellizzer, A. Benvenuti, B. Gleixner, Y. Kim, B. Johnson, M. Magistretti, T. Marangon, A. Pirovano, R. Bex, and G. Atwood. A 90nm phase change memory technology for stand-alon non-volatile memory applications. 2006 Symposium on VLSI Technology Digest of Technical Papers, 2006.

[151] Numonyx to present phase change memory research results at leading technology industry conference. Website, December 2009. http://www.numonyx.com/enUS/About/PressRoom/Releases/Pages/PCMResearchResults.aspx.

[152] Richard L. McCreery. Molecular electronic junctions. The Electrochemical Society Interface, pages 46-51, 2004.

[153] Ali Osman Solak, Srikanth Ranganathan, Takashi Itoh, and Richard L. McCreery. A mechanism for conductance switching in carbon-based molecular electronic junctions. Electrochemical and Solid-State Letters, 5(8):E43-E46, 2002.

[154] Srikanth Ranganathan and Richard L. McCreery. Electroanalytical performance of carbon films with near-atomic flatness. Analytical Chemistry, 73(5):893-900, 2001.

[155] Marshall I. Nathan, Jr. John E. Smith, and K.N. Tu. Raman spectra of glassy carbon. Journal of Applied Physics, 45(5):2370, 1974. 
[156] A.O. Solak, L.R. Eichorst, W.J. Clark, and R.L. McCreery. Modified carbon surfaces as 'organic electrodes' that exhibit conductance switching. Analytical Chemistry, 75:296-305, 2003.

[157] A.M. Nowak and R.L. McCreery. Characterization of carbon/nitroazobenzene/titanium molecular electronic junctions with photoelectron and Raman spectrpscopy. Anal. Chem., 76:10891097, 2004.

[158] W.C. Bigelow, D.L. Pickett, and W.A. Zisman. Oleophobic monolayers. i. films adsorbed from solution in non-polar liquids. J. Colloid Sci., 1(6):513-538, 1946.

[159] Abraham Ulman. Formation and structure of self-assembled monolayers. Chem. Rev., 96:1533$1554,1996$.

[160] Daniel K. Schwartz. Mechanisms and kinetics of self-assembled monolayer formation. Annu. Rev. Phys. Chem., 52:107-137, 2001.

[161] Ralph G. Nuzzo and David L. Allara. Adsorption of bifunctional organic disulfides on gold surfaces. J. Am. Chem. Soc., 105(3):4481-4483, 1983.

[162] C. Vericat, M.E. Vela, G.A Benitez, J.A. Martin Gago, X. Torrelles, and R.C. Salvarezza. Surface characterization of sulfur and alkanethiol self-assembled monolayers on $\mathrm{Au}(111) . J$. Phys.: Condens. Matter, 18:R867-R900, 2006.

[163] Ja-Ryong Koo, Sang-Woo Pyo, Jun-Ho Kim, Hyun-Koo Lee, and Young Kwan Kim. Self assembled monolayer for molecular electronics. Jpn. J. Appl. Phys., 44(1B):566-569, 2005.

[164] Shailender Kolipaka, Rajendra K. Aithal, and Debasish Kuila. Fabrication and characterization of an indium tin oxide-octadecanethiol-aluminum junction for molecular electronics. Appl. Phys. Lett., 88(23):233104, 2006.

[165] André P. Labonté, Steven L. Tripp, Ronald Reifenberger, and Alexander Wei. Scanning tunneling spectroscopy of insulating self-assembled monolayers on $\mathrm{Au}(111)$. J. Phys. Chem. $B, 106(34): 8721-8725,2002$.

[166] Hyunwook Song, Youngsang Kim, Yun Hee Jang, Heejun Jeong, Mark A. Reed, and Takhee Lee. Observation of molecular orbital gating. Nature, 462:1039-1043, 2009. 
[167] Ravi Chandra Chintala. Towards molecular and nanoscale devices by octadecanethiol self assembled monolayer and boron nanowires. Master's thesis, Michigan Technological University, 2008.

[168] D. Porath, Y. Goldstein, A. Grayevsky, and O. Millo. Scanning tunneling microscopy studies of annealing of gold films. Surface Science, 321(1-2):81-88, 1994.

[169] Jun Zhou, David Bullen, Xuefeng Wang, and Chang Liu. Conductivity-based contact sensing for probe arrays in dip-pen nanolithography. Applied Physics Letters, 83(3):581-583, 2003.

[170] Jeremy M. Beebe and James G. Kushmerick. Nanoscale switch elements from self-assembled monolayers on silver. Applied Physics Letters, 90:083117, 2007.

[171] Marc J. Madou. Fundamentals of microfabrication: the science of miniaturization. CRC Press, second edition, 2002.

[172] L.F. Thompson, C.G. Wilson, and M.J. Bowden, editors. Introduction to Microlithography - Theory, Materials and Processing, chapter 1. ACS Symposium Series. American Chemical Society, Washington, DC, 1983.

[173] P. Rai-Choudhury, editor. Handbook for Microlithography, Micromachining and Microfabrication, volume 1: Microlithography. SPIE Optical Engineering Press, 1997.

[174] Ewen Smith and Geoffrey Dent. Modern Raman Spectroscopy - A Practical Approach. John Wiley \& Sons, Ltd., 2005.

[175] H. Hiura, T.W. Ebbesen, K. Tanigaki, and H. Takahashi. Raman studies of carbon nanotubes. Chem. Phys. Lett., 202:509, 1993.

[176] Joseph Goldstein, Dale Newbury, David Joy, Charles Lyman, Patrick Echlin, Eric Lifshin, Linda Sawyer, and Joseph Michael. Scanning electron microscopy and X-Ray Microanalysis. Klewer Academic/Plenum Publishers, New York, NY, third edition, 2003. 